\title{
A Multiple-Curve Lévy Forward Rate Model in a Two-Price Economy
}

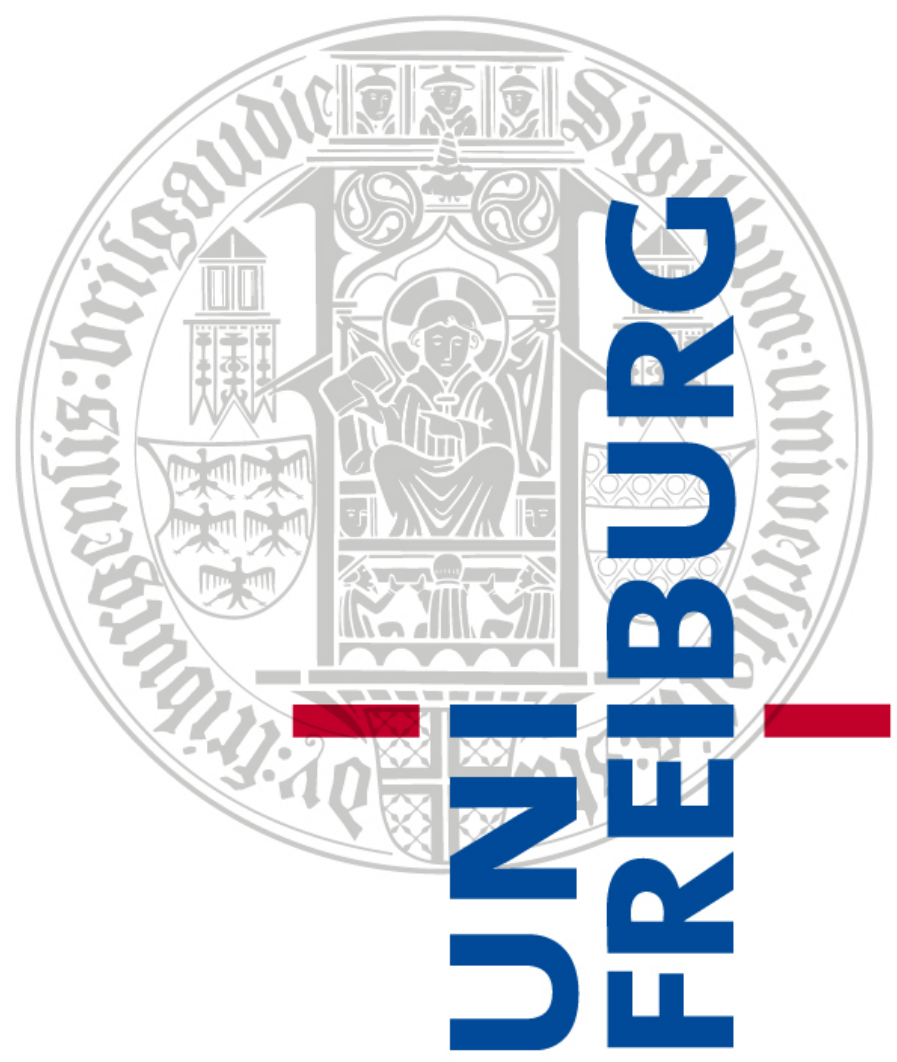

Dissertation zur Erlangung des Doktorgrades der Fakultät für Mathematik und Physik

der Albert-Ludwigs-Universität Freiburg im Breisgau

vorgelegt von

Christoph Gerhart

Juni 2016 
Dekan: $\quad$ Prof. Dr. Dietmar Kröner

Referenten: Prof. Dr. Ernst Eberlein

Prof. Dr. Zorana Grbac

Datum der Promotion: 21. Juli 2016

Albert-Ludwigs-Universität Freiburg

Abteilung für Mathematische Stochastik

Eckerstraße 1

D-79104 Freiburg

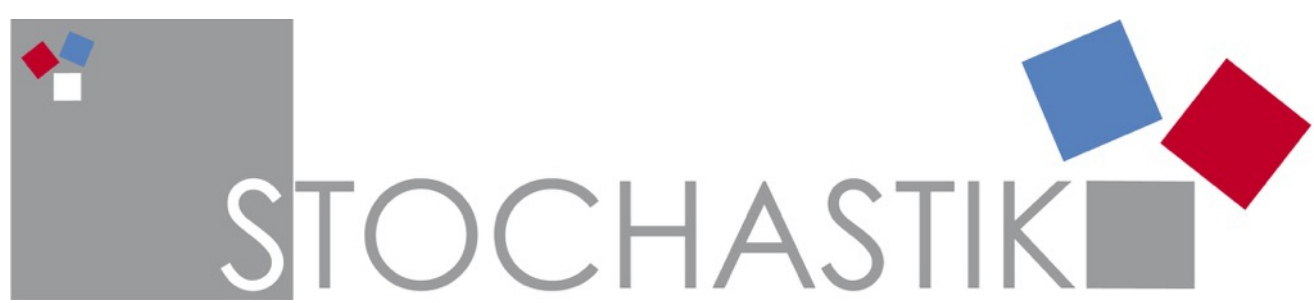


To My Beloved Parents 

ABSTRACT. In this thesis, we combine and merge the multiple-curve approach and the two-price theory based on acceptability indices in a Lévy interest rate model.

A multiple-curve Heath-Jarrow-Morton (HJM) forward rate model driven by time-inhomogeneous Lévy processes (a multiple-curve Lévy term structure model) is presented. We find deterministic conditions which ensure the monotonicity of the curves. Explicit valuation formulas for some interest rate derivatives are established, namely forward rate agreements, swaps, caps, floors and digital options. These formulas can numerically be evaluated very fast by using the Fourier based valuation method. Furthermore, we apply the two-price theory to this multiple-curve setting. Ask and bid model prices of caplets, floorlets and digital options are derived.

A general procedure how to calibrate this two-price multiple-curve interest rate model to market data is described. As a practical application, the model is calibrated to market prices of caps for dates before and after the global financial crisis. 

CONTENTS

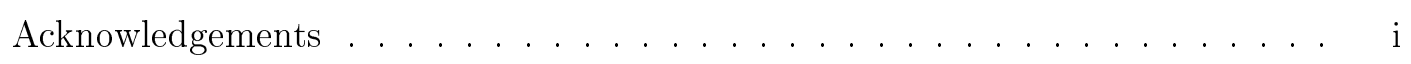

Introduction . . . . . . . . . . . . . . . . . . . . . . . 2

Overview - Synopsis . . . . . . . . . . . . . . . . 3

Practical Implementation . . . . . . . . . . . . . . . . . . 3

1. Mathematical Preliminary $\mathbf{5}$

1.1. Driving Process of the Model . . . . . . . . . . . . . . . . . . 5

1.2. Credit Risk Modelling . . . . . . . . . . . . . . . . . . 8

1.2.1. Enlargements of Filtrations and the Martingale Invariance Property 8

1.2.2. Canonical Construction of Conditionally Independent Default Times 11

\begin{tabular}{|l|l|}
\hline 2. Two-Price Theory in Multiple-Curve Term Structure Models & 17
\end{tabular}

2.1. Two-Price Theory Based on Acceptability Indices . . . . . . . . . . . . . 18

2.1.1. Coherent Utility Functions . . . . . . . . . . . . . . . . 18

2.1.2. Fundamental Examples of Coherent Utility Functions . . . . . . . 19

2.1.3. Acceptability Indices . . . . . . . . . . . . . . . . . 24

2.1.4. Weighted Value at Risk Acceptability Index . . . . . . . . . . . 26

2.1.5. Ask and Bid Prices . . . . . . . . . . . . . . . . . 29

2.2. Multiple-Curve Interest Rate Term Structure Modelling . . . . . . . . . . 30

2.2.1. Fundamental Model Approaches . . . . . . . . . . . . . . . . 31

2.2.2. Interest Rate Derivatives . . . . . . . . . . . . . . . . . . . . 33

2.2.3. The Multiple-Curve Approach. . . . . . . . . . . . . . . . . . . 35

2.2.4. Valuation Formulas of Interest Rate Derivatives . . . . . . . . . . . 42

2.3. Application of the Two-Price Theory . . . . . . . . . . . . . . . . 52

2.4. Calibration Procedure . . . . . . . . . . . . . . . . . . . . 52 
3. The Multiple-Curve Lévy Forward Rate Model with Application of the TwoPrice Theory $\quad \mathbf{5 5}$

3.1. The Multiple-Curve Lévy Forward Rate Model . . . . . . . . . . . . . . . 55

3.1.1. Discount Curve . . . . . . . . . . . . . . . . . 55

$3.1 .2 . \quad$ Multiple Curves . . . . . . . . . . . . . . . . . . . 61

3.2. Monotonicity of the Curves . . . . . . . . . . . . . . 68

3.2.1. The Monotonicity Condition . . . . . . . . . . . . 68

3.2.2. A Suitable Model Framework . . . . . . . . . . . . . . . . . 71

3.3. Valuation Formulas with Application of the Two-Price Theory . . . . . . . 77

3.3.1. Valuation Formulas of Interest Rate Derivatives . . . . . . . . . . . 77

3.3.2. Application of the Two-Price Theory . . . . . . . . . . . . . 88

$\begin{array}{lr}\text { 4. Model Calibration } & 99\end{array}$

4.1. Description and Processing of Cap and Floor Market Data . . . . . . . . . 99

4.2. Calibration of Caps in the Two-Price and Single-Curve Setting . . . . . 103

4.2 .1$. Data Sets . . . . . . . . . . . . . . . . . 103

4.2.2. Calibration Procedure . . . . . . . . . . . . . . . . . . . 103

4.2.3. Model Framework (I) . . . . . . . . . . . . . . . . 106

4.2.4. Calibration Results of Model Framework (I) . . . . . . . . . . . . . 111

$4.2 .5 . \quad$ Model Framework (II) $\ldots \ldots$. . . . . . . . . . . . . . . 113

4.2.6. Calibration Results of Model Framework (II) . . . . . . . . . . . . 117

4.3. Calibration of Caps in the Two-Price and Multiple-Curve Setting . . . . . 118

4.3 .1$. Data Sets . . . . . . . . . . . . . . . . . . 118

4.3.2. Calibration Procedure . . . . . . . . . . . . . . . . . . . . 119

4.3.3. Model Framework . . . . . . . . . . . . . . . . . . . . . . 119

4.3.4. Calibration Results . . . . . . . . . . . . . . . . . . . 130

\begin{tabular}{ll}
\hline Appendices & 131
\end{tabular}

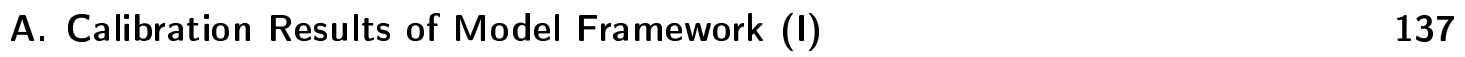

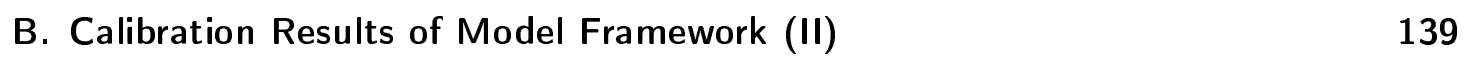

\begin{tabular}{|l|l|}
\hline C. Calibration Results in the Multiple-Curve Setting & 141
\end{tabular}

\begin{tabular}{ll}
\hline Bibliography & 151
\end{tabular} 


\section{Acknowledgements}

First and foremost, I would like to thank my academic teacher and supervisor Prof. Dr. Ernst Eberlein. He introduced me to the topic of Lévy term structure models and aroused my interest for the multiple-curve approach and the two-price theory. Furthermore, his valuable advice and support in our pleasant discussions have been essential to finish this thesis.

I would also like to express my thanks to my dear colleagues at the Department of Mathematical Stochastics at the University of Freiburg for making the past couple of years so exciting and valuable. Specifically, I would like to mention the mathematical and non-mathematical discussions with Prof. Dr. Thorsten Schmidt, Prof. Dr. Ludger Rüschendorf, Prof. Dr. Hans Rudolf Lerche, Prof. Dr. Eva Lütkebohmert-Holtz, Dr. Volker Pohl, Dr. Ernst August Frhr. v. Hammerstein, Dr. Andrej Depperschmidt, Dr. Dominik Stich, Dr. Victor Wolf, Dr. Swen Kiesel, Benedikt Köpfer, Peter Czuppon, Benedikt Vogt and (the good soul of our department) Monika Hattenbach. Special thanks go to Dr. Patrick Bäurer, Jonathan Ansari, Marcus Rudmann and Felix Hermann for their valuable comments on the manuscript. I would also like to show my gratitude to Annkatrin Burkart for the linguistic advice.

From the bottom of my heart, I would like to thank my family and friends for their lovely support over all the years. Especially, I would like to emphasise my parents Anni and Hubert, my brother Björn with Marion, Louis and Jule and my other brother Julian. 



\section{Introduction}

In answer to the changes in fixed income markets due to the global financial crisis in the summer of 2007, practitioners and researchers established the so-called multiplecurve approach. Basically, the post-crisis market situation can be explained by taking into account credit and liquidity risk. But instead of developing a complicated and sophisticated model that considers credit and liquidity effects, one prefers using this more practical approach.

The multiple-curve approach is based on the consideration of an individual discount curve and as many term structures of interest rates as occurring tenors. The term structure of interest rates, also known as the yield curve, is the function that relates the yield on a zero-coupon bond to maturity (continuously compounded rate of return from holding a zero-coupon bond over a period of time; called yield-to-maturity). A tenor represents the duration of a compounding period. By the one-to-one correspondence between the bond price and its yield-to-maturity, the term structure of interest rates can be represented either by the yield curve or by the function that relates the bond price to maturity (bond price curve). In this thesis, each term structure is interpreted as the bond price curve. Therefore, we often use the term curve as an analogy for the notion of term structure.

In the multiple-curve setting, interest rates are divided into different risk classes depending on their related tenor (fixed income market segmentation). This means that each tenor-related term structure reflects the respective (credit and liquidity) risk of the corresponding tenor-dependent interest rates. The discount curve is specifically used to determine the present value of a (future) payoff. It can be interpreted as the risk-free curve.

In the post-crisis situation, a bootstrapping technique for the construction of the different initial curves was developed. Furthermore, the question concerning the stochastic modelling of the dynamics of the various tenor-dependent interest rates arises. A logically similar method to the one used for the initial multiple-curve construction was applied. Generally speaking, the interest rate curves are modelled jointly but distinctly. Consequently, a large number of short-rate models, Heath-Jarrow-Morton-based models and market models related to the multiple-curve approach were developed. We refer to this type of models as multiple-curve interest rate models.

We present a multiple-curve interest rate model based on the framework of HeathJarrow-Morton. A discount curve and a finite set of risky curves which take into account credit and liquidity issues are modelled. We use time-inhomogeneous Lévy processes as driving processes of the model. On the one hand, their generality related to the theory of semimartingales are of special interest from a mathematical point of view. On the other hand, these processes are also appropriate for practical purposes because of their distributional flexibility and relatively simple handling. 
The differences between the curves originate from the individual included risks. In general, curves corresponding to larger tenors represent more credit and liquidity risk than curves related to smaller tenors. This fact leads to a crucial monotonicity condition of the curves. More specifically, the larger the related tenor (the risk) of a (fictitious risky) bond is, the cheaper it is. The discount bond is therefore the most expensive one. One of our key objectives is the development of a model framework which guarantees this monotonicity. We derive deterministic conditions on the model quantities, which results in additional restrictions on the model parameters.

Furthermore, liquidity risk can cause a large spread between ask and bid prices of a derivative. This situation is also observed in fixed income market data during the financial crisis. On this account, we apply the two-price theory based on acceptability indices to our multiple-curve interest rate model. Ask and bid prices of some financial products are derived and we present a method to evaluate them numerically.

The question how to calibrate our two-price multiple-curve model to market data arises. A general calibration procedure that deals with this fact is described. To illustrate this issue, we calibrate the model to market data for dates before and during the global financial crisis. The calibration results are also demonstrated in detail.

\section{Overview - Synopsis}

This thesis consists of four chapters and three appendices. It is organised as follows: Chapter 1 deals with the mathematical tools and concepts that are needed in order to develop our model. We review time-inhomogeneous Lévy processes which we use as driving processes of the model. The theoretical background for this topic is given by Jacod and Shiryaev [56]. Furthermore, we consider the mathematical modelling of credit risk. We investigate the enlargements of filtrations and the martingale invariance property. Based on these issues, we study the construction of conditionally independent default times with regard to our multiple-curve approach. The works of Brémaud and Yor [16], Jeanblanc and Le Cam [59] and Bielecki and Rutkowski [12] are crucial for this study.

In chapter 2, we introduce the theory of two prices based on the concept of acceptability indices. Ask and bid prices for financial positions related to a static period of time are derived. We use the paper of Cherny and Madan [22] and Eberlein and Madan [35] as main reference. We also present the fundamental theory of interest rate term structure modelling. In particular, the multiple-curve approach is motivated by market data that are observed since the beginning of the global financial crisis in the summer of 2007 . We extensively explain this modern model approach. The application of the two-price theory to the multiple-curve approach is also described. Finally, we present a general calibration procedure in this two-price and multiple-curve setting. 
Chapter 3 introduces our multiple-curve Lévy forward rate model. This model approach bases on the work of Eberlein and Raible [38], Eberlein and Kluge [33] and Crépey, Grbac, and Nguyen [23]. Moreover, we apply the two-price theory to this model. Valuations formulas for some interest rate derivatives are established.

In chapter 4, we calibrate our model to market data for dates before and during the financial crisis. The accurate framework and the calibration results are stated.

In the appendices, we present the calibration results in form of the relative errors between market and model prices.

\section{Practical Implementation}

For practical purposes, we apply the programming language and environment $\mathrm{R}$ for the graphics as well as for the statistical and numerical computations. The used packages are mentioned at the end of the thesis. The presented data sets of this work are based on data provided by Bloomberg. 



\section{MATHEMATICAL PRELIMINARY}

In this chapter, some basic mathematical tools and concepts that are needed to develop our model are stated. We first introduce the driving process that we will use: Time-inhomogeneous Lévy processes. The mathematical theory for these processes is essentially developed by Jacod and Shiryaev [56]. Therefore, we mainly adapt the notation of these authors. The use of time-inhomogeneous Lévy processes in financial models is justified by their great distributional flexibility (cf. Eberlein and Keller [32, Eberlein and Raible [38], Eberlein [29], Eberlein and Kluge [34], Eberlein, Grbac, and Schmidt 42] and Eberlein, Grbac, and Schmidt [41]).

We also address the mathematical modelling of credit risk. More specifically, we deal with the enlargement of filtrations and the martingale invariance property. This topic is intensively studied by Brémaud and Yor [16], Jeanblanc and Le Cam [59] and Bielecki and Rutkowski [12]. Then, we present a method for the canonical construction of conditionally independent default times. This approach is of particular importance for our type of multiple-curve Heath-Jarrow-Morton model.

\subsection{Driving Process of the Model}

Let $T^{*} \in \mathbb{R}_{+}:=[0, \infty)$ be a finite time horizon and $\mathscr{B}:=\left(\Omega, \mathscr{G}, \mathbb{F}=\left(\mathscr{F}_{t}\right)_{t \in\left[0, T^{*}\right]}, P\right)$ be a stochastic basis that satisfies the usual conditions (or, equivalently, is complete) in the sense of Jacod and Shiryaev [56, Definition I.1.2 and Definition I.1.3]:

- The stochastic basis $\mathscr{B}$ is defined as probability space $(\Omega, \mathscr{G}, P)$ endowed with an increasing and right-continuous family $\mathbb{F}=\left(\mathscr{F}_{t}\right)_{t \in\left[0, T^{*}\right]}$ of sub- $\sigma$-fields of $\mathscr{G}$. The family $\mathbb{F}=\left(\mathscr{F}_{t}\right)_{t \in\left[0, T^{*}\right]}$ is called filtration and $\mathscr{B}$ is also named as filtered probability space. By convention, one usually sets $\mathscr{F}_{T^{*}}=\mathscr{G}$.

- The stochastic basis $\mathscr{B}$ is said to satisfy the usual conditions (or, equivalently, is 


\section{Mathematical Preliminary}

called complete) if the $\sigma$-field $\mathscr{G}$ is $P$-complete and if every $\mathscr{F}_{t}$ contains all $P$-null sets of $\mathscr{G}$.

As driving process, we consider a $d$-dimensional time-inhomogeneous Lévy process $L=\left(L^{1}, \ldots, L^{d}\right)^{\top}$ on $\mathscr{B}$ with $L^{i}=\left(L_{t}^{i}\right)_{t \in\left[0, T^{*}\right]}$ for every $i \in\{1, \ldots, d\}$ with $d \in \mathbb{N}$. This means that $L$ is an $\mathbb{F}$-adapted process with independent increments and absolutely continuous characteristics (abbreviated by PIIAC) defined on $\mathscr{B}$ (see Eberlein, Jacod, and Raible [39] and Jacod and Shiryaev [56]). Such type of stochastic process is also known as additive process (see Sato [81]). We emphasise that $L$ is a $d$-dimensional semimartingale (see also Jacod and Shiryaev [56, §5.]).

We can assume that the paths of each component of $L$ are càdlàg (continue à droite avec des limites à gauche). This means that these paths are right-continuous and admit left-hand limits (almost surely). We also postulate that each component $L^{i}$ starts in zero. The law of $L_{t}$ is determined by its characteristic function:

$$
\begin{aligned}
\mathbb{E}\left[e^{i\left\langle u, L_{t}\right\rangle}\right]=\exp \left(\int _ { 0 } ^ { t } \left[i\left\langle u, b_{s}(h)\right\rangle-\frac{1}{2}\left\langle u, c_{s} u\right\rangle\right.\right. \\
\left.\left.\quad+\int_{\mathbb{R}^{d}}\left(e^{i\langle u, x\rangle}-1-i\langle u, h(x)\rangle\right) F_{s}(d x)\right] d s\right) \quad\left(u \in \mathbb{R}^{d}\right) .
\end{aligned}
$$

Here, $h$ is a truncation function in $\mathscr{C}_{t}^{d}, b_{s}(h)=\left(b_{s}^{1}(h), \ldots, b_{s}^{d}(h)\right)^{\top}:\left[0, T^{*}\right] \rightarrow \mathbb{R}^{d}$, $c_{s}=\left(c_{s}^{i j}\right)_{i, j \leq d}:\left[0, T^{*}\right] \rightarrow \mathbb{R}^{d \times d}$ whose values are in the set of symmetric nonnegativedefinite $d \times d$-matrices and $F_{s}$ is a Lévy measure for every $s \in\left[0, T^{*}\right]$, i.e. a nonnegative measure on $\left(\mathbb{R}^{d}, \mathcal{B}\left(\mathbb{R}^{d}\right)\right)$ that integrates $\left(|x|^{2} \wedge 1\right)$ and satisfies $F_{s}(\{0\})=0$ for every $s \in\left[0, T^{*}\right]$. As truncation function, one usually takes $h(x)=x \cdot \mathbb{1}_{\{|x| \leq 1\}}$. We denoted by $\langle\cdot, \cdot\rangle$ the Euclidean scalar product on $\mathbb{R}^{d}$ and $|\cdot|$ is the corresponding norm. The scalar product on $\mathbb{R}^{d}$ is extended to complex numbers by setting $\langle w, z\rangle:=\sum_{j=1}^{d} w_{j} z_{j}$ for every $w, z \in \mathbb{C}^{d}$. Thus, $\langle\cdot, \cdot\rangle$ is not the Hermitian scalar product here. We further assume that

$$
\int_{0}^{T^{*}}\left[\left|b_{s}(h)\right|+\left\|c_{s}\right\|+\int_{\mathbb{R}^{d}}\left(|x|^{2} \wedge 1\right) F_{s}(d x)\right] d s<\infty,
$$

where $\|\cdot\|$ denotes any norm on the set of $d \times d$-matrices. The triplet $(b, c, F)=$ $\left(b_{s}, c_{s}, F_{s}\right)_{s \in\left[0, T^{*}\right]}$ represents the local characteristics of $L$. We also make the following standing assumption on the (exponential) moments throughout this thesis:

Assumption $(\mathbb{E M})$ : There are constants $M, \epsilon>0$, such that, for every

$$
u \in[-(1+\epsilon) M,(1+\epsilon) M]^{d},
$$


we have

$$
\int_{0}^{T^{*}} \int_{\{|x|>1\}} \exp \langle u, x\rangle F_{s}(d x) d s<\infty .
$$

Assumption $(\mathbb{E M})$ is equivalent to $\mathbb{E}\left[\exp \left\langle u, L_{t}\right\rangle\right]<\infty$ for all $t \in\left[0, T^{*}\right]$ and $u \in$ $[-(1+\epsilon) M,(1+\epsilon) M]^{d}$. We will consider interest rate models with underlying processes that are exponentials of stochastic integrals with respect to $L$. By the theory of riskneutral pricing, these underlying processes have to be (local) martingales under the risk-neutral measure. Therefore, they have to have finite expectations which is exactly guaranteed by assumption $(\mathbb{E M})$. A direct consequence of $(\mathbb{E M})$ is that the random variable $L_{t}$ has finite expectation. Therefore, the representation 1.1 can be written as

$$
\begin{aligned}
\mathbb{E}\left[e^{i\left\langle u, L_{t}\right\rangle}\right]=\exp \left(\int _ { 0 } ^ { t } \left[i\left\langle u, b_{s}\right\rangle-\frac{1}{2}\left\langle u, c_{s} u\right\rangle\right.\right. \\
\left.\left.\quad+\int_{\mathbb{R}^{d}}\left(e^{i\langle u, x\rangle}-1-i\langle u, x\rangle\right) F_{s}(d x)\right] d s\right) .
\end{aligned}
$$

We emphasise that the characteristic $b$ is now different from the one in 1.1) (see Sato [81, chapter 2, section 8]). In this thesis, we will always work with the local characteristics $(b, c, F)$ that are derived from form 1.2 . Another implication of assumption $(\mathbb{E M})$ is that the process $L$ is even a special semimartingale. Thus, its canonical representation is given by the simple form

$$
L_{t}=\int_{0}^{t} b_{s} d s+\int_{0}^{t} \sqrt{c_{s}} d W_{s}+\int_{0}^{t} \int_{\mathbb{R}^{d}} x\left(\mu^{L}-\nu\right)(d s, d x)
$$

(see Jacod and Shiryaev [56, Corollary II.2.38]), where $W=\left(W_{t}\right)_{t \in\left[0, T^{*}\right]}$ is a standard $d$-dimensional Brownian motion (Wiener process), $\sqrt{c_{s}}$ is a measurable version of the square root of $c_{s}$, and $\mu^{L}$ is the random measure of jumps of $L$ with compensator $\nu(d s, d x)=F_{s}(d x) d s$. Obviously, the integrals in (1.3) should be understood componentwise. We stress that assumption $(\mathbb{E M})$ is valid for all processes that we are interested in. In particular, it holds for processes that are generated by generalised hyperbolic distributions. The (extended) cumulant process associated with process $L$ is denoted by $\theta_{s}$ and given by

$$
\theta_{s}(z)=\left\langle z, b_{s}\right\rangle+\frac{1}{2}\left\langle z, c_{s} z\right\rangle+\int_{\mathbb{R}^{d}}\left(e^{\langle z, x\rangle}-1-\langle z, x\rangle\right) F_{s}(d x)
$$

for every $z \in \mathbb{C}^{d}$ where this function is defined. A detailed analysis of the cumulant process for semimartingales is given by Kallsen and Shiryaev [61. Note that if $L$ is 


\section{Mathematical Preliminary}

a (homogeneous) Lévy process, i.e. if the increments of $L$ are stationary, the triplet $\left(b_{s}, c_{s}, F_{s}\right)$ and thus also $\theta_{s}$ do not depend on $s$. In this case, we write $\theta$ for short. It then equals the cumulant (also called log moment generating function) of $L_{1}$. Observe that the cumulant process is related to a specific measure. Below, which measure is meant can unambiguously be seen from the notation.

\subsection{Credit Risk Modelling}

Our multiple-curve model takes credit risk into consideration. To this end, we present some related topics that we need to develop this framework. More specifically, we give a detailed explanation of the enlargement of filtrations and the corresponding martingale invariance property. Then, the canonical construction of a finite set of conditionally independent default times is stated.

\subsubsection{Enlargements of Filtrations and the Martingale Invariance Property}

Let us consider a stochastic basis $\left(\Omega, \mathscr{G}, \mathbb{F}=\left(\mathscr{F}_{t}\right)_{t \in \mathbb{R}_{+}}, P\right)$ that satisfies the usual conditions. By an enlargement of the filtration $\mathbb{F}$ we mean a filtration $\mathbb{G}=\left(\mathscr{G}_{t}\right)_{t \in \mathbb{R}_{+}}$defined on the same probability space $(\Omega, \mathscr{G}, P)$ such that

(i) $\mathscr{F}_{t} \subset \mathscr{G}_{t}$ for every $t \in \mathbb{R}_{+}$and

(ii) the stochastic basis $\left(\Omega, \mathscr{G}, \mathbb{G}=\left(\mathscr{G}_{t}\right)_{t \in \mathbb{R}_{+}}, P\right)$ satisfies the usual conditions.

The family $\mathbb{F}$ is called reference filtration. Originally, the right-continuity of $\mathbb{G}$ is not explicitly requested in the definition of an enlargement of a filtration. For technical reasons, we require this condition but we stress that in the remaining part of this subsection all the claims can be stated without this additional property.

According to Brémaud and Yor [16, section 2.4.], the martingale invariance (or immersion) property is classically stated as any of the following equivalent hypotheses:

$(\mathcal{H} 1)$ Every square integrable $\mathbb{F}$-martingale is a square integrable $\mathbb{G}$-martingale.

$(\mathcal{H} 2)$ Every bounded $\mathbb{F}$-martingale is a bounded $\mathbb{G}$-martingale.

$(\mathcal{H} 3)$ Every $\mathbb{F}$-local martingale is a $\mathbb{G}$-local martingale.

The martingale invariance property reveals a nice structure of the enlargement $\mathbb{G}$ relative to the reference filtration $\mathbb{F}$ (see Brémaud and Yor [16, Theorem 3]). In this context, the notion of conditional independence of two families of sets with respect to a given $\sigma$-field plays an important role. Although we generally use this concept in the case where all the considered families are $\sigma$-fields, it is beneficial for our further approach to define it in a slightly broader sense. To this end, let us consider a probability space 
$(F, \mathcal{F}, Q)$. We call two families of sets $\mathcal{A}$ and $\mathcal{B}$ with $\mathcal{A}, \mathcal{B} \subset \mathcal{F}$ conditionally independent given a sub- $\sigma$-field $\mathcal{S}$ of $\mathcal{F}$ if

$$
Q(A \cap B \mid \mathcal{S})=Q(A \mid \mathcal{S}) Q(B \mid \mathcal{S}) \quad \text { a.s. for all } A \in \mathcal{A}, B \in \mathcal{B} \text {. }
$$

We symbolically write $\mathcal{A} \perp \mathcal{B} \mid \mathcal{S}$ if the relation 1.4 is valid.

Lemma 1.2.1 For two families of sets $\mathcal{A}$ and $\mathcal{B}$ that are closed under formation of intersections, we have

$$
\mathcal{A} \perp \mathcal{B}|\mathcal{S} \Longrightarrow \sigma(\mathcal{A}) \perp \sigma(\mathcal{B})| \mathcal{S} \text {. }
$$

Proof: For every set $E \in \mathcal{F}$, we define

$$
\mathcal{D}_{E}:=\{G \in \mathcal{F} \mid Q(E \cap G \mid \mathcal{S})=Q(E \mid \mathcal{S}) Q(G \mid \mathcal{S}) \text { a.s. }\} .
$$

One easily shows that $\mathcal{D}_{E}$ is a Dynkin system. Clearly, if $A \in \mathcal{A}$, we have $\mathcal{B} \subset \mathcal{D}_{A}$. From Dynkins $\pi$ - $\lambda$-Theorem (for details see Klenke [63, Satz 1.19]), it follows that $\sigma(\mathcal{B})=$ $\delta(\mathcal{B}) \subset \mathcal{D}_{A}$. By the symmetry property of the conditional independence of two sets, this fact can be reformulated as $\mathcal{A} \subset \mathcal{D}_{B}$ for every $B \in \sigma(\mathcal{B})$. By using Dynkins $\pi$ - $\lambda$-Theorem again, we obtain $\sigma(\mathcal{A}) \subset \mathcal{D}_{B}$ for every $B \in \sigma(\mathcal{B})$.

In the case where $\mathcal{A}$ and $\mathcal{B}$ are $\sigma$-fields, condition $(1.4$ can equivalently be written as

$$
\mathbb{E}_{Q}[\xi \eta \mid \mathcal{S}]=\mathbb{E}_{Q}[\xi \mid \mathcal{S}] \mathbb{E}_{P}[\eta \mid \mathcal{S}] \text { a.s. }
$$

for any bounded, $\mathcal{A}$-measurable random variable $\xi$ and any bounded, $\mathcal{B}$-measurable random variable $\eta$. This fact is based on the monotone convergence Theorem for conditional expectations. Another crucial argument related to the martingale invariance property is a result coming from Dellacherie and Meyer [26]: For three sub- $\sigma$-fields $\mathcal{A}_{1}, \mathcal{A}_{2}$ and $\mathcal{A}_{3}$ of $\mathcal{F}$, it holds that $\mathcal{A}_{1}$ and $\mathcal{A}_{3}$ are conditionally independent given $\mathcal{A}_{2}$ under $Q$ if and only if

$$
\mathbb{E}_{Q}\left[X_{3} \mid \mathcal{A}_{1} \vee \mathcal{A}_{2}\right]=\mathbb{E}_{Q}\left[X_{3} \mid \mathcal{A}_{2}\right]
$$

for every bounded and $\mathcal{A}_{3}$-measurable random variable $X_{3}$ (cf. Grbac [50, Theorem 2.3.]), where we define $\mathcal{A}_{1} \vee \mathcal{A}_{2}:=\sigma\left(\mathcal{A}_{1}, \mathcal{A}_{2}\right)$. Observe that this statement can easily be extended to the set of integrable $\mathcal{A}_{3}$-measurable random variables by standard truncation and approximation arguments (for instance, consider $X_{3, n}=\max \left\{X_{3}, n\right\}$ for $n \in \mathbb{N}$ ). The following equivalent forms of the hypotheses above are obtained by the result of Dellacherie and Meyer [26]:

$(\mathcal{H} 4)$ For every $t \in \mathbb{R}_{+}$, the $\sigma$-fields $\mathscr{F}_{\infty}$ and $\mathscr{G}_{t}$ are conditionally independent given $\mathscr{F}_{t}$ under $P$. 
$(\mathcal{H} 5)$ For any $t \in \mathbb{R}_{+}$and any bounded, $\mathscr{F}_{\infty}$-measurable random variable $\xi$, we have

$$
\mathbb{E}_{P}\left[\xi \mid \mathscr{G}_{t}\right]=\mathbb{E}_{P}\left[\xi \mid \mathscr{F}_{t}\right]
$$

$(\mathcal{H} 6)$ For any $t \in \mathbb{R}_{+}$and any bounded, $\mathscr{G}_{t}$-measurable random variable $\eta$, we have

$$
\mathbb{E}_{P}\left[\eta \mid \mathscr{F}_{\infty}\right]=\mathbb{E}_{P}\left[\eta \mid \mathscr{F}_{t}\right]
$$

We mention that Bielecki and Rutkowski [12, chapter 6] state the martingale invariance property as follows:

$(\mathcal{H} 7)$ Every $\mathbb{F}$-martingale is a $\mathbb{G}$-martingale.

It is shown that $(\mathcal{H} 7)$ is equivalent to $(\mathcal{H} 4)$ (see Bielecki and Rutkowski [12, Lemma 6.1.1.]).

There are basically two ways to enlarge a filtration (cf. Jeanblanc and Le Cam [59] and Bielecki and Rutkowski [12]):

1. Initial enlargement: $\mathscr{G}_{t}=\mathscr{F}_{t} \vee \mathscr{H}$ for a sub- $\sigma$-field $\mathscr{H}$ of $\mathscr{G}$.

2. Progressive enlargement: $\mathscr{G}_{t}=\mathscr{F}_{t} \vee \mathscr{H}_{t}$ for a filtration $\mathbb{H}=\left(\mathscr{H}_{t}\right)_{t \in \mathbb{R}_{+}}$defined on $(\Omega, \mathscr{G}, P)$.

Kusuoka [65] proves by means of a counterexample that the martingale invariance property is generally not invariant with respect to an equivalent change of the underlying probability measure. However, this fact does not pose a serious problem in our setting. Concretely, let us consider two probability measures $P$ and $Q$ defined on the filtered space $\left(\Omega, \mathscr{G}, \mathbb{G}=\left(\mathscr{G}_{t}\right)_{t \in \mathbb{R}_{+}}\right)$where $Q$ is locally absolutely continuous with respect to $P$, i.e. $Q$ is absolutely continuous with respect to $P$ on $\left(\Omega, \mathscr{G}_{t}\right)$ for every $t \in \mathbb{R}_{+}$(shortly written as $Q \stackrel{\text { loc }}{\ll} P$ ). Then, by Jacod and Shiryaev [56, Theorem III.3.4], there exists a Radon-Nikodym density process $Z=\left(Z_{t}\right)_{t \in \mathbb{R}_{+}}$with

$$
\frac{d Q_{\left.\right|_{\mathscr{G}_{t}}}}{d P_{\mathscr{G}_{t}}}=Z_{t}
$$

for all $t \in \mathbb{R}_{+}$. If the density process $Z$ is $\mathbb{F}$-adapted the martingale invariance property holds after change of measure (see also Jamshidian [57]). To see this, let $M$ be an $(\mathbb{F}, Q)$ martingale. Since $Z$ is $\mathbb{F}$-adapted we conclude that $M Z$ is an $(\mathbb{F}, P)$-martingale by an application of Jacod and Shiryaev [56, Proposition III.3.8]. The martingale invariance property is valid for $(\mathbb{F}, \mathbb{G})$ under $P$. Thus, $M Z$ is a $(\mathbb{G}, P)$-martingale and it follows that $M$ is a $(\mathbb{G}, Q)$-martingale by applying Jacod and Shiryaev [56, Proposition III.3.8] once again. 


\subsubsection{Canonical Construction of Conditionally Independent Default Times}

The aim of this subsection is the construction of a finite set of default times. They are used in the multiple-curve term structure model to specify the credit risk components. To this end, let $\hat{\mathscr{B}}=\left(\hat{\Omega}, \hat{\mathscr{F}}, \hat{\mathbb{F}}=\left(\hat{\mathscr{F}}_{t}\right)_{t \in \mathbb{R}_{+}}, P\right)$ be a complete stochastic basis (recall Jacod and Shiryaev [56, Definition I.1.2 and I.1.3]) and $m \in \mathbb{N}^{*}:=\{1,2,3, \ldots\}$. The probability measure $P$ will play the role of a local martingale measure in the model. Let $\Gamma^{1}, \ldots, \Gamma^{m}$ be real-valued, $\hat{\mathbb{F}}$-adapted, continuous and increasing stochastic processes defined on $\hat{\mathscr{B}}$. It is assumed that $\Gamma_{0}^{k}=0$ and $\Gamma_{\infty}^{k}:=\lim _{t \uparrow \infty} \Gamma_{t}^{k}=\infty$ for every $k \in\{1, \ldots, m\}$. We consider an auxiliary probability space $(\tilde{\Omega}, \tilde{\mathscr{F}}, \tilde{P})$ endowed with a family of independent random variables $\xi_{1}, \ldots, \xi_{m}$ that are uniformly distributed on the interval $[0,1]$. This situation can canonically be achieved by considering the product space $(\tilde{\Omega}, \tilde{\mathscr{F}}, \tilde{P})=$ $\left(\chi_{k=1}^{m} \tilde{\Omega}_{k}, \bigotimes_{k=1}^{m} \tilde{\mathscr{F}}_{k}, \bigotimes_{k=1}^{m} \tilde{P}_{k}\right)$ and setting $\xi_{k}=p_{k}$ for every $k \in\{1, \ldots, m\}$ with

$$
p_{k}:\left\{\begin{array}{l}
\tilde{\Omega} \rightarrow \tilde{\Omega}_{k} \\
\tilde{\omega}=\left(\tilde{\omega}_{1}, \ldots, \tilde{\omega}_{m}\right) \mapsto \tilde{\omega}_{k}
\end{array}\right.
$$

and $\left(\tilde{\Omega}_{k}, \tilde{\mathscr{F}}_{k}, \tilde{P}_{k}\right)=(\mathbb{R}, \mathcal{B}(\mathbb{R}), \mathcal{U})$, where we denote by $\mathcal{U}$ the uniform distribution on $[0,1]$ (cf. Bauer [9, chapter II]).

We state the product space

$$
\left(\Omega, \mathscr{G}, P^{\mathrm{d}}\right):=(\hat{\Omega} \times \tilde{\Omega}, \hat{\mathscr{F}} \otimes \tilde{\mathscr{F}}, P \otimes \tilde{P})
$$

and denote by $\mathbb{F}=\left(\mathscr{F}_{t}\right)_{t \in\left[0, T^{*}\right]}$ the trivial extension of $\hat{\mathbb{F}}$ to the enlarged probability space $\left(\Omega, \mathscr{G}, P^{\mathrm{d}}\right)$. This means that each $A \in \mathscr{F}_{t}$ is of the form $\hat{A} \times \tilde{\Omega}$ for some $\hat{A} \in \hat{\mathscr{F}}_{t}$. Observe that $\mathbb{F}$ is right-continuous and denotes the reference filtration here. All the random variables (functions) and stochastic processes defined on $\hat{\mathscr{B}}$ and $(\tilde{\Omega}, \tilde{\mathscr{F}}, \tilde{P})$ are extended to the enlarged filtered probability space $\left(\Omega, \mathscr{G}, \mathbb{F}=\left(\mathscr{F}_{t}\right)_{t \in \mathbb{R}_{+}}, P^{\mathrm{d}}\right)$ in the usual canonical way. We retain their names when we consider them on this complete stochastic basis to avoid unnecessary and confusing notation (cf. Eberlein and Özkan [36] and Kluge [64, section 4.2]). Observe that every $(\hat{\mathbb{F}}, P)$-(local) martingale is also a $\left(\mathbb{F}, P^{\mathrm{d}}\right)$-(local) martingale.

For every $k \in\{1, \ldots, m\}$, let us define a random time $\tau^{k}: \Omega \rightarrow \mathbb{R}_{+}$on $\left(\Omega, \mathscr{G}, P^{\mathrm{d}}\right)$ by setting

$$
\tau^{k}:=\inf \left\{t \in \mathbb{R}_{+} \mid e^{-\Gamma_{t}^{k}} \leq \xi_{k}\right\}=\inf \left\{t \in \mathbb{R}_{+} \mid \Gamma_{t}^{k} \geq \eta_{k}\right\},
$$

where the random variable $\eta_{k}:=-\ln \xi_{k}$ is exponentially distributed with mean one under $P^{\mathrm{d}}$. Obviously, $\eta_{1}, \ldots, \eta_{m}$ is a family of independent random variables. For every $k \in\{1, \ldots, m\}$, we denote by $\mathbb{H}^{k}=\left(\mathscr{H}_{t}^{k}\right)_{t \in \mathbb{R}_{+}}$the right-continuous filtration generated 


\section{Mathematical Preliminary}

by the default process $H^{k}=\left(H_{t}^{k}\right)_{t \in \mathbb{R}_{+}}$that is defined by $H_{t}^{k}=\mathbb{1}_{\left\{\tau^{k} \leq t\right\}}$, i.e. $\mathscr{H}_{t}^{k}=$ $\sigma\left(H_{u}^{k}: 0 \leq u \leq t\right)=\sigma\left(\left\{\tau^{k} \leq u\right\}: 0 \leq u \leq t\right)$.

Now, we want to enlarge the reference filtration $\mathbb{F}$. Unfortunately, the filtration $\tilde{\mathbb{G}}=$ $\left(\tilde{\mathscr{G}}_{t}\right)_{t \in \mathbb{R}_{+}}$induced by $\tilde{\mathscr{G}}_{t}:=\mathscr{F}_{t} \vee \mathscr{H}_{t}^{1} \vee \cdots \vee \mathscr{H}_{t}^{m}:=\sigma\left(\mathscr{F}_{t}, \mathscr{H}_{t}^{1}, \ldots, \mathscr{H}_{t}^{m}\right.$ ) (written as $\tilde{\mathbb{G}}=\mathbb{F} \vee \mathbb{H}^{1} \vee \cdots \vee \mathbb{H}^{m}$ ) does not have to be right-continuous (cf. Song [86]). Therefore, we endow the probability space $\left(\Omega, \mathscr{G}, P^{\mathrm{d}}\right)$ with the filtration $\mathbb{G}=\left(\mathscr{G}_{t}\right)_{t \in \mathbb{R}_{+}}$given by

$$
\mathscr{G}_{t}:=\bigcap_{s>t} \tilde{\mathscr{G}}_{s}, \quad \text { for any } t \in \mathbb{R}_{+} .
$$

This filtration trivially satisfies the right-continuity. Since the stochastic basis $\left(\Omega, \mathscr{G}, \mathbb{F}, P^{\mathrm{d}}\right)$ is complete (see also Jacod and Shiryaev [56, §1a. 1.4]) it easily follows that the enlarged stochastic basis $\left(\Omega, \mathscr{G}, \mathbb{G}=\left(\mathscr{G}_{t}\right)_{t \in \mathbb{R}_{+}}, P^{\mathrm{d}}\right)$ is also complete. We conclude that $\mathbb{G}$ is specified as the smallest enlargement of $\mathbb{F}$ containing $\tilde{\mathbb{G}}$.

We notice that $\tilde{\mathscr{G}}_{t}$ represents all information available at date $t$ including the observations of all random times $\tau^{1}, \ldots, \tau^{m}$. Formally, we have the following Lemma:

Lemma 1.2.2 For any $t \in \mathbb{R}_{+}$, the $\sigma$-field $\tilde{\mathscr{G}}_{t}$ is given by

$$
\begin{aligned}
\tilde{\mathscr{G}}_{t} & =\mathscr{F}_{t} \vee \sigma\left(\left\{\tau^{j} \leq t_{j}\right\}: j \in\{1, \ldots, m\}, 0 \leq t_{j} \leq t\right) \\
& =\mathscr{F}_{t} \vee \sigma\left(\left\{\tau^{j}>t_{j}\right\}: j \in\{1, \ldots, m\}, 0 \leq t_{j} \leq t\right) \\
& =\mathscr{F}_{t} \vee \sigma\left(\bigcap_{j \in J}\left\{\tau^{j}>t_{j}\right\}: J \subset\{1, \ldots, m\}, 0 \leq t_{j} \leq t \forall j \in J\right) .
\end{aligned}
$$

Proof: Obviously, it is sufficient to show that

$$
\begin{aligned}
\mathscr{H}_{t}^{1} \vee \cdots \vee \mathscr{H}_{t}^{m} & =\sigma\left(\left\{\tau^{j} \leq t_{j}\right\}: j \in\{1, \ldots, m\}, 0 \leq t_{j} \leq t\right) \\
& =\sigma\left(\left\{\tau^{j}>t_{j}\right\}: j \in\{1, \ldots, m\}, 0 \leq t_{j} \leq t\right) \\
& =\sigma\left(\bigcap_{j \in J}\left\{\tau^{j}>t_{j}\right\}: J \subset\{1, \ldots, m\}, 0 \leq t_{j} \leq t \forall j \in J\right) .
\end{aligned}
$$

By definition, we have $\mathscr{H}_{t}^{1} \vee \cdots \vee \mathscr{H}_{t}^{m}=\sigma\left(\bigcup_{j=1}^{m} \sigma\left(\left\{\tau^{j} \leq t_{j}\right\}: 0 \leq t_{j} \leq t\right)\right)$. For any $k \in\{1, \ldots, m\}$, it clearly holds

$$
\mathscr{H}_{t}^{k}=\sigma\left(\left\{\tau^{k} \leq t_{k}\right\}: 0 \leq t_{k} \leq t\right) \subset \sigma\left(\left\{\tau^{j} \leq t_{j}\right\}: j \in\{1, \ldots, m\}, 0 \leq t_{j} \leq t\right)
$$


and, therefore, we obtain

$$
\bigcup_{j=1}^{m} \sigma\left(\left\{\tau^{j} \leq t_{j}\right\}: 0 \leq t_{j} \leq t\right) \subset \sigma\left(\left\{\tau^{j} \leq t_{j}\right\}: j \in\{1, \ldots, m\}, 0 \leq t_{j} \leq t\right) .
$$

Let $k \in\{1, \ldots, m\}$ and $t_{k} \in \mathbb{R}_{+}$with $0 \leq t_{k} \leq t$ be chosen. Since $\left\{\tau^{k} \leq t_{k}\right\} \in \mathscr{H}_{t}^{k}$, it is evident that

$$
\left\{\tau^{k} \leq t_{k}\right\} \in \bigcup_{j=1}^{m} \sigma\left(\left\{\tau^{j} \leq t_{j}\right\}: 0 \leq t_{j} \leq t\right)
$$

The second and third equality are immediately clear by definition.

Note that the generator of the $\sigma$-field $\mathscr{H}_{t}:=\mathscr{H}_{t}^{1} \vee \cdots \vee \mathscr{H}_{t}^{m}$

$$
\mathcal{E}:=\left\{\bigcap_{j \in J}\left\{\tau^{j}>t_{j}\right\}: J \subset\{1, \ldots, m\}, 0 \leq t_{j} \leq t \forall j \in J\right\}
$$

is closed under formation of intersections. It is evident that the random times $\tau^{1}, \ldots, \tau^{m}$ are $\mathbb{G}$-stopping times but we stress that they may fail to be $\mathbb{F}$-stopping times. We explicitly assume that $\tau^{1}, \ldots, \tau^{m}$ are not $\mathbb{F}$-stopping times. Since $\Gamma_{t}^{k}$ is $\mathscr{F}_{\infty}$-measurable and $\xi_{k}$ is independent of $\mathscr{F}_{\infty}$ on $\left(\Omega, \mathscr{G}, P^{\mathrm{d}}\right)$ for every $k \in\{1, \ldots, m\}$, we obtain by using Kallenberg [60, Theorem 6.4] that

$$
P^{\mathrm{d}}\left(\tau^{k}>t \mid \mathscr{F}_{\infty}\right)=P^{\mathrm{d}}\left(e^{-\Gamma_{t}^{k}}>\xi_{k} \mid \mathscr{F}_{\infty}\right)=\tilde{P}\left(e^{-x}>\xi_{k}\right)_{\mid x=\Gamma_{t}^{k}}=e^{-\Gamma_{t}^{k}} .
$$

Consequently, we conclude from the $\mathscr{F}_{t}$-measurability of the last term that

$$
P^{\mathrm{d}}\left(\tau^{k}>t \mid \mathscr{F}_{t}\right)=P^{\mathrm{d}}\left(P^{\mathrm{d}}\left(\tau^{k}>t \mid \mathscr{F}_{\infty}\right) \mid \mathscr{F}_{t}\right)=e^{-\Gamma_{t}^{k}}
$$

for each $t \in \mathbb{R}_{+}$. Therefore, the stochastic process $\Gamma^{k}$ represents the $\mathbb{F}$-hazard process of $\tau^{k}$ under $P^{\mathrm{d}}$ (see Bielecki and Rutkowski [12, Definition 8.2.1.]) and the $\left(\mathbb{F}, \tilde{\mathbb{G}}^{k}\right)$ martingale hazard process of $\tau^{k}$ under $P^{\mathrm{d}}$, where we set $\tilde{\mathbb{G}}^{k}:=\mathbb{F} \vee \mathbb{H}^{k}$ (see Bielecki and Rutkowski [12, Definition 6.1.1. and Lemma 8.2.2.]). More specifically, the process $M^{k}=\left(M_{t}^{k}\right)_{t \in \mathbb{R}_{+}}$given by

$$
M_{t}^{k}=H_{t}^{k}-\Gamma_{t \wedge \tau^{k}}^{k}
$$

is a $\tilde{\mathbb{G}}^{k}$-martingale under $P^{\mathrm{d}}$. One verifies that it is also a $\widetilde{\mathbb{G}}$-martingale under $P^{\mathrm{d}}$ (cf. Bielecki and Rutkowski [12, Lemma 9.1.1.]).

By a straightforward modification of Bielecki and Rutkowski [12, Lemma 9.1.1.] and a further application of Kallenberg [60, Theorem 6.4], we obtain for each subset $J \subset$ 
$\{1, \ldots, m\}$ and every $T>0$ satisfying $0 \leq t_{j} \leq T$ for any $j \in J$ that

$$
P^{\mathrm{d}}\left(\bigcap_{j \in J}\left\{\tau^{j}>t_{j}\right\} \mid \mathscr{F}_{\infty}\right)=P^{\mathrm{d}}\left(\bigcap_{j \in J}\left\{\tau^{j}>t_{j}\right\} \mid \mathscr{F}_{T}\right)=\prod_{j \in J} e^{-\Gamma_{t_{j}}^{j}}=e^{-\sum_{j \in J} \Gamma_{t_{j}}^{j}}
$$

In particular, for any $k \in\{1, \ldots, m\}$ and every $0 \leq t_{k} \leq T$ it holds

$$
P^{\mathrm{d}}\left(\tau^{k}>t_{k} \mid \mathscr{F}_{\infty}\right)=P^{\mathrm{d}}\left(\tau^{k}>t_{k} \mid \mathscr{F}_{T}\right)=e^{-\Gamma_{t_{k}}^{k}}
$$

We conclude that the random times $\tau^{1}, \ldots, \tau^{m}$ are (dynamically) conditionally independent with respect to filtration $\mathbb{F}$ under $P^{\mathrm{d}}$ (see Bielecki and Rutkowski [12, Definition 9.1.1. and Definition 9.1.2.]). We stress that these properties are additional features of the canonical construction of the random times $\tau^{1}, \ldots, \tau^{m}$ which means that they are not necessarily valid in a general framework.

Next, we examine if the martingale invariance property for $\mathbb{F}$ and $\mathbb{G}$ is satisfied.

Lemma 1.2.3 For any $t \in \mathbb{R}_{+}$, the $\sigma$-fields $\mathscr{F}_{\infty}$ and $\mathscr{H}_{t}=\mathscr{H}_{t}^{1} \vee \cdots \vee \mathscr{H}_{t}^{m}$ are conditionally independent given $\mathscr{F}_{t}$ under $P^{\mathrm{d}}$.

Proof: Let $A \in \mathscr{F}_{\infty}$ and $B \in \mathcal{E}$. Then, there is a subset $J \subset\{1, \ldots, m\}$ and $0 \leq t_{j} \leq t$ for any $j \in J$ such that $B=\bigcap_{j \in J}\left\{\tau^{j}>t_{j}\right\}$ and we get

$$
\begin{aligned}
& P^{\mathrm{d}}\left(A \cap \bigcap_{j \in J}\left\{\tau^{j}>t_{j}\right\} \mid \mathscr{F}_{t}\right)=\mathbb{E}_{P^{\mathrm{d}}}\left[\mathbb{1}_{A} \mathbb{1}_{\bigcap_{j \in J}\left\{\tau^{j}>t_{j}\right\}} \mid \mathscr{F}_{t}\right] \\
& =\mathbb{E}_{P^{\mathrm{d}}}\left[\mathbb{E}_{P^{\mathrm{d}}}\left[\mathbb{1}_{A} \mathbb{1}_{\bigcap_{j \in J}\left\{\tau^{j}>t_{j}\right\}} \mid \mathscr{F}_{\infty}\right] \mid \mathscr{F}_{t}\right]=\mathbb{E}_{P^{\mathrm{d}}}\left[\mathbb{1}_{A} P^{\mathrm{d}}\left(\bigcap_{j \in J}\left\{\tau^{j}>t_{j}\right\} \mid \mathscr{F}_{\infty}\right) \mid \mathscr{F}_{t}\right] \\
& =P^{\mathrm{d}}\left(A \mid \mathscr{F}_{t}\right) P^{\mathrm{d}}\left(\bigcap_{j \in J}\left\{\tau^{j}>t_{j}\right\} \mid \mathscr{F}_{t}\right) .
\end{aligned}
$$

Then, the statement follows from Lemma 1.2.1

Consequently, it follows that the martingale invariance property is fulfilled in a framework where we consider $\mathbb{F}$ and $\tilde{\mathbb{G}}$. To see this, let $M$ be an $\mathbb{F}$-martingale under $P^{\mathrm{d}}$. Obviously, $M$ is $\tilde{\mathbb{G}}$-adapted and, for $t, s \in \mathbb{R}_{+}$with $s \leq t$, we have

$$
\mathbb{E}_{P^{\mathrm{d}}}\left[M_{t} \mid \tilde{\mathscr{G}}_{s}\right]=\mathbb{E}_{P^{\mathrm{d}}}\left[M_{t} \mid \mathscr{F}_{s} \vee \mathscr{H}_{s}^{1} \vee \cdots \vee \mathscr{H}_{s}^{m}\right]=\mathbb{E}_{P^{\mathrm{d}}}\left[M_{t} \mid \mathscr{F}_{s}\right]=M_{s}
$$

and therefore $(\mathcal{H} 7)$ holds for $\mathbb{F}$ and $\tilde{\mathbb{G}}$. The martingale invariance property should be valid for $\mathbb{F}$ and the enlargement $\mathbb{G}$. To show this, let us consider a bounded $\mathbb{F}$-martingale $M, s, t \in \mathbb{R}_{+}$with $s \leq t$ and $A \in \mathscr{G}_{s}=\bigcap_{u>s} \tilde{\mathscr{G}}_{u}$. Clearly, $M$ is $\mathbb{G}$-adapted and it holds 
that $A \in \tilde{\mathscr{G}}_{u}$ for every $u$ satisfying $s<u<t$. For each $\tilde{u} \in(s, t)$, we have

$$
\int_{A} \mathbb{E}_{P^{\mathrm{d}}}\left[M_{t} \mid \mathscr{G}_{s}\right] d P^{\mathrm{d}}=\int_{A} M_{t} d P^{\mathrm{d}}=\int_{A} \mathbb{E}_{P^{\mathrm{d}}}\left[M_{t} \mid \tilde{\mathscr{G}}_{\tilde{u}}\right] d P^{\mathrm{d}}=\int_{A} M_{\tilde{u}} d P^{\mathrm{d}}
$$

from which we conclude that

$$
\int_{A} M_{t} d P^{\mathrm{d}}=\int_{A} M_{\tilde{u}} d P^{\mathrm{d}}
$$

for every $A \in \mathscr{G}_{s}$. Then, by taking the limit $\tilde{u} \downarrow s$ on both sides and applying the dominated convergence Theorem, we obtain

$$
\int_{A} \mathbb{E}_{P^{\mathrm{d}}}\left[M_{t} \mid \mathscr{G}_{s}\right] d P^{\mathrm{d}}=\int_{A} M_{t} d P^{\mathrm{d}}=\int_{A} M_{s} d P^{\mathrm{d}}
$$

where we used the right-continuity of the process. Hence, $M$ is also a bounded $\mathbb{G}$ martingale and from hypothesis $(\mathcal{H} 2)$ we obtain the validity of the martingale invariance property with respect to filtration $\mathbb{F}$ and each enlargement $\hat{\mathbb{G}}$ of $\mathbb{F}$ satisfying $\hat{\mathbb{G}} \subset \mathbb{G}$. Analogously, one shows that the process $M^{k}$ in 1.5 is also a $\mathbb{G}$-martingale.

In the following Lemma, we study the behaviour of a time-inhomogeneous Lévy process defined on the original stochastic basis after enlarging it.

Lemma 1.2.4 Let $L$ be a d-dimensional time-inhomogeneous Lévy process with semimartingale characteristics $(B, C, \nu)=\left(\int_{0}^{\cdot} b_{s} d s, \int_{0}^{\cdot} c_{s} d s, F_{t} \otimes d t\right)$ defined on the stochastic basis $(\hat{\Omega}, \hat{\mathscr{F}}, \hat{\mathbb{F}}, P)$ (see Jacod and Shiryaev [56, Definition II.2.6]). Then, it is also a d-dimensional time-inhomogeneous Lévy process with characteristics $(B, C, \nu)$ on the enlarged stochastic basis $\left(\Omega, \mathscr{G}, \mathbb{G}, P^{\mathrm{d}}\right)$.

Proof: The process $L$ is clearly $\mathbb{G}$-adapted, càdlàg and starts in zero. For any $t \in \mathbb{R}_{+}$, one verifies that the characteristic function of $L_{t}$ is preserved with respect to $P^{\mathrm{d}}$. To see this, we have

$$
\begin{aligned}
\mathbb{E}_{P^{\mathrm{d}}}\left[\exp \left(i\left\langle u, L_{t}\right\rangle\right)\right] & =\int_{\hat{\Omega} \times \tilde{\Omega}} \exp \left(i\left\langle u, L_{t}(\hat{\omega}, \tilde{\omega})\right\rangle\right) d(P \otimes \tilde{P})(\hat{\omega}, \tilde{\omega}) \\
& =\int_{\hat{\Omega}} \exp \left(i\left\langle u, L_{t}(\hat{\omega})\right\rangle\right) d P(\hat{\omega})=\mathbb{E}_{P}\left[\exp \left(i\left\langle u, L_{t}\right\rangle\right)\right] .
\end{aligned}
$$

Hence, it follows that the characteristics of $L$ are invariant with respect to the enlargement. We finally show that the increment $L_{t}-L_{s}$ is independent of $\mathscr{G}_{s}$ for every $0 \leq s<t$. 


\section{Mathematical Preliminary}

Let $A \in \mathscr{G}_{s}$ and $B \in \mathcal{B}\left(\mathbb{R}^{d}\right)$ be chosen. Then, we obtain

$$
\begin{aligned}
& P^{\mathrm{d}}\left(A \cap\left\{\left(L_{t}-L_{s}\right) \in B\right\}\right)=\int_{A} \mathbb{1}_{B}\left(L_{t}-L_{s}\right) d P^{\mathrm{d}}=\int_{A} \mathbb{E}_{P^{\mathrm{d}}}\left[\mathbb{1}_{B}\left(L_{t}-L_{s}\right) \mid \mathscr{G}_{s}\right] d P^{\mathrm{d}} \\
& =\int_{A} \mathbb{E}_{P^{\mathrm{d}}}\left[\mathbb{1}_{B}\left(L_{t}-L_{s}\right) \mid \mathscr{F}_{s}\right] d P^{\mathrm{d}}=\int_{A} \mathbb{E}_{P^{\mathrm{d}}\left[\mathbb{1}_{B}\left(L_{t}-L_{s}\right)\right] d P^{\mathrm{d}}} \\
& =P^{\mathrm{d}}(A) P^{\mathrm{d}}\left(\left\{\left(L_{t}-L_{s}\right) \in B\right\}\right),
\end{aligned}
$$

where we used hypothesis $(\mathcal{H} 5)$.

It is often convenient to postulate that each random time $\tau^{k}$ possesses an $\mathbb{F}$-intensity (or $\mathbb{F}$-hazard rate) $\gamma^{k}$ (see Bielecki and Rutkowski [12, chapter 5 and 8]). This means that the hazard process $\Gamma^{k}$ of $\tau^{k}$ admits the integral representation

$$
\Gamma_{t}^{k}=\int_{0}^{t} \gamma_{s}^{k} d s
$$

for any $t \in \mathbb{R}_{+}$and some non-negative, $\mathbb{F}$-progressively measurable stochastic process $\gamma^{k}$ with integrable sample paths. Note that we obtain

$$
M_{t}^{k}=H_{t}^{k}-\int_{0}^{t \wedge \tau^{k}} \gamma_{s}^{k} d s=H_{t}^{k}-\int_{0}^{t} \mathbb{1}_{\left\{\tau^{k} \geq s\right\}} \gamma_{s}^{k} d s=H_{t}^{k}-\int_{0}^{t} \mathbb{1}_{\left\{\tau^{k}>s\right\}} \gamma_{s}^{k} d s
$$

for any $k \in\{1, \ldots, m\}$. 


\section{TWO-PRICE THEORY IN MULTIPLE-CURVE TERM STRUCTURE MODELS}

In this chapter, we present the two-price theory based on acceptability indices developed by Cherny and Madan [22]. In the classical theory of mathematical finance, the time- $t$ arbitrage-free price $\Pi_{t}(X)$ of a claim with discounted payoff $X$ is determined by the linear valuation formula

$$
\Pi_{t}(X)=\mathbb{E}_{Q}\left[X \mid \mathscr{F}_{t}\right]
$$

Here, $Q$ is a risk-neutral probability measure defined on a filtered measurable space $\left(\Omega,\left(\mathscr{F}_{t}\right)_{t \geq 0}, \mathscr{F}\right)$ and $X \in L^{1}(\Omega, \mathscr{F}, Q)$ (see Delbaen and Schachermayer [25]). In general, the term 2.1 is assumed to be the valuation formula of the price for which we sell and buy the financial product in liquid markets. But financial products that are traded in illiquid markets typically have two prices: The one for buying from the market, called ask price, and the other one for selling to the market, referred to as bid price. Here the market is considered as a passive counterparty (cf. Cherny and Madan [22]). It follows that the prices are allowed to vary with the trading direction. Typically, the ask price is above the bid price. In this setting, we obtain non-linear valuation formulas for the ask and bid prices. The mid price is the price between the ask and the bid price. In general, the mid price can be interpreted as the average of the current bid and ask prices being quoted. But we stress that, in some cases, it is rounded up or down to the nearest valid tradable price for convenience purposes, and it is therefore not the exact average. Of course, ask and bid market quotes of derivatives also differ in liquid markets but their spreads are often seen as negligible.

We stress that our meaning of the spread should not be confused with the bid-ask spread relating to transaction costs or other frictions (see also the comments of Eberlein [30]). The effect of liquidity risk can cause a large bid-ask spread. More specifically, the 


\section{Two-Price Theory in Multiple-Curve Term Structure Models}

risk of trading and holding a position, especially in illiquid markets, influences the ask and bid prices in such a way that they diverge substantially. This situation was observed in fixed income markets with the beginning of the financial crisis in the summer of 2007. The two-price theory provides a mathematical framework to deal with this issue. Therefore, all these facts motivate the application of the two-price theory to the multiple-curve term structure model approach. The concept of the multiple-curve approach is introduced in detail. It is motivated by a variety of market anomalies that arise with the beginning of the crisis. Finally, a method to calibrate a model to market data in this two-price multiple-curve setting is described.

\subsection{Two-Price Theory Based on Acceptability Indices}

We review the theory of two prices that is based on the concept of acceptability indices. A detailed explanation about its origin and development is given. In particular, we state the crucial connection between coherent utility functions and distortion functions. The (Weighted Value at Risk) acceptability index is defined and we specify the corresponding ask and bid prices of a financial product.

\subsubsection{Coherent Utility Functions}

Let us consider a period of time between two fixed dates $t$ and $T$. These points in time are seen as today $(t=0)$ and as a finite time horizon $(T>0)$. A financial position transforms initial wealth $X_{0} \in \mathbb{R}$ into some random future payoff $X_{T}$. Consequently, we comprehend $X_{T}$ as a real-valued random variable defined on a given probability space $(\Omega, \mathscr{F}, P)$. The space of random variables on $(\Omega, \mathscr{F}, P)$ with values in $\mathbb{R}$ is denoted by $L^{0}$.

Below, $X_{T}$ is interpreted as the discounted payoff at time 0 . To measure the risk of financial positions, one can use the concept of coherent risk measures and acceptance sets (acceptance cones) developed by Artzner, Delbaen, Eber, and Heath [4] and Delbaen 24. A coherent risk measure $\rho$ is defined as monotone, cash invariant, convex and positively homogeneous map on the space of ( $P$-almost surely) bounded, real-valued random variables $L^{\infty}$ (for details see Artzner, Delbaen, Eber, and Heath [4] or Cherny [20, Definition 2.1]). A risk measure $\rho$ associates with each discounted payoff $X_{T}$ a number $\rho\left(X_{T}\right) \in \mathbb{R}$ that quantifies the risk of the financial product. More specifically, $\rho\left(X_{T}\right)$ is understood as the amount of money (i.e. the capital requirement) that should be added to $X_{T}$ in order to make the position acceptable for an investor. A financial position with discounted payoff $X_{T}$ is therefore called acceptable if and only if $\rho\left(X_{T}\right) \leq 0$. To simplify the notation, we prefer considering the coherent utility function $u:=-\rho$ instead of the corresponding coherent risk measure $\rho$. The acceptance set (acceptance cone) of $u$ is defined as the set $\mathcal{A}_{u}:=\left\{X \in L^{\infty} \mid u(X) \geq 0\right\}$. A coherent utility function $u$ has the 
Fatou property, i.e. if $\left|X_{n}\right| \leq 1, X_{n} \stackrel{P}{\rightarrow} X$, then $\lim \sup _{n} u\left(X_{n}\right) \leq u(X)$ (cf. Cherny [20, Definition 2.1 (e)]), if and only if there exists a non-empty set $\mathcal{D} \subset \mathcal{M}_{1}(P)$ such that

$$
u(X)=\inf _{Q \in \mathcal{D}} \mathbb{E}_{Q}[X]
$$

where $\mathcal{M}_{1}(P)$ denotes the space of probability measures on $(\Omega, \mathscr{F})$ that are absolutely continuous with respect to $P$ (cf. Delbaen [24] and Föllmer and Schied [46, Chapter 4]). By using this representation, Cherny [20] extends the definition of a coherent utility function from the space $L^{\infty}$ to $L^{0}$. To be precise, a map $u: L^{0} \rightarrow \overline{\mathbb{R}}:=[-\infty, \infty]$ is called coherent utility function on $L^{0}$ if it has a representation $(2.2)$, where $\mathbb{E}_{Q}[X]$ is understood as $\mathbb{E}_{Q}\left[X^{+}\right]-\mathbb{E}_{Q}\left[X^{-}\right]$with convention $\infty-\infty=-\infty$. One easily sees that this extension is consistent with the definition of coherent utility functions on $L^{\infty}$. In an analogous way, we define the acceptance set of a coherent utility function on $L^{0}$. Note that a set $\mathcal{D}$ for which the representation above holds is not unique. The largest set is given by $\mathcal{D}_{m}(u):=\left\{Q \in \mathcal{M}_{1}(P) \mid \mathbb{E}_{Q}[X] \geq u(X)\right.$ for every $\left.X \in L^{0}\right\}$ and is called determining set of $u$. Clearly, the determining set of a coherent utility function is convex. For coherent utility functions on $L^{\infty}$ it is also $L^{1}$-closed. We stress that each measure from $\mathcal{M}_{1}(P)$ is identified with its Radon-Nikodym density with respect to $P$ and the topological structure of $L^{1}$ is used. For a convex $L^{1}$-closed subset $\mathcal{D} \subset \mathcal{M}_{1}(P)$, we define a coherent utility function $u$ by $u(X):=\inf _{Q \in \mathcal{D}} \mathbb{E}_{Q}[X]$. Then, by the Theorem of HahnBanach, it can be shown that $\mathcal{D}=\mathcal{D}_{m}(u)$. For a coherent utility function $u$ on $L^{0}$ we refer to $L_{w}^{1}(u):=\left\{X \in L^{0} \mid u(X)>-\infty\right.$ and $\left.u(-X)>-\infty\right\}$ as the weak $L^{1}$-space of $u$. Clearly, we have $u(X) \in \mathbb{R}$ for every $X \in L_{w}^{1}(u)$.

\subsubsection{Fundamental Examples of Coherent Utility Functions}

Let us introduce some crucial examples of coherent utility functions that are studied by Föllmer and Schied [46, Chapter 4] and Cherny [20, Chapter 2]. First, we consider the Expected Shortfall $u^{\mathrm{ES}_{\lambda}}$ (Average Value at Risk or Tail Value at Risk) defined by

$$
u^{\mathrm{ES}_{\lambda}}:\left\{\begin{array}{l}
L^{0} \rightarrow \overline{\mathbb{R}} \\
X \mapsto \inf _{Q \in \mathcal{D}_{\lambda}} \mathbb{E}_{Q}[X]
\end{array}\right.
$$

where $\lambda \in(0,1]$ and

$$
\mathcal{D}_{\lambda}=\left\{Q \in \mathcal{M}_{1}(P) \mid \frac{d Q}{d P} \leq \lambda^{-1}\right\}
$$

We set $u^{\mathrm{ES}_{0}}(X):=\operatorname{essinf}(X)$ for $\lambda=0$. Since $\mathcal{D}_{\lambda}$ is a convex and $L^{1}$-closed subset of $\mathcal{M}_{1}(P)$, we conclude that $\mathcal{D}_{\lambda}$ is the determining set of $u^{\mathrm{ES}_{\lambda}}$. Let us denote by $q_{\lambda}(X)$ the right $\lambda$-quantile of $X \in L^{0}$, i.e. $q_{\lambda}(X):=\inf \{x \in \mathbb{R} \mid P(X \leq x)>\lambda\}$. Cherny [20, 


\section{Two-Price Theory in Multiple-Curve Term Structure Models}

Proposition 2.6] shows that, for $\lambda \in(0,1]$ and $X \in L^{0}, u^{\mathrm{ES}_{\lambda}}$ can be represented by

$$
u^{\mathrm{ES}_{\lambda}}(X)=\lambda^{-1} \int_{\left(-\infty, q_{\lambda}(X)\right)} x P_{X}(d x)+c_{\lambda} q_{\lambda}(X)
$$

where $c_{\lambda}:=1-\lambda^{-1} P_{X}\left(-\infty, q_{\lambda}(X)\right)$ and $P_{X}$ denotes the distribution of $X$ with respect to $P$. For a random variable $X$ with continuous cumulative distribution function we obtain

$$
u^{\mathrm{ES}_{\lambda}}(X)=\lambda^{-1} \int_{\left(-\infty, q_{\lambda}(X)\right)} x P_{X}(d x) .
$$

The Weighted Value at Risk is a generalisation of the Expected Shortfall. The basic idea behind this concept is to weight the values of $u^{\mathrm{ES}_{\lambda}}(X)$ by a probability measure. More precisely, the Weighted Value at Risk utility function $u^{\mathrm{W}_{\mu}}$ on $L^{\infty}$ is defined by

$$
u^{\mathrm{W}_{\mu}}:\left\{\begin{array}{l}
L^{\infty} \rightarrow \mathbb{R} \\
X \mapsto \int_{[0,1]} u^{\mathrm{ES}_{\lambda}}(X) \mu(d \lambda),
\end{array}\right.
$$

where $\mu$ is a probability measure on $([0,1], \mathcal{B}([0,1]))$. It can also be extended to $L^{0}$ in the standard way (cf. previous subsection). To this end, we define

$$
u^{\mathrm{W}_{\mu}}:\left\{\begin{array}{l}
L^{0} \rightarrow \overline{\mathbb{R}} \\
X \mapsto \inf _{Q \in D_{\mu}} \mathbb{E}_{Q}[X],
\end{array}\right.
$$

where $D_{\mu}$ is the determining set of $u^{W_{\mu}}$ on $L^{\infty}$. The justification of this definition can be understood by the following observations: $u^{\mathrm{W}_{\mu}}$ on $L^{\infty}$ is a coherent utility function satisfying the Fatou property. Hence, the set $D_{\mu}$ is convex and $L^{1}$-closed and is therefore equal to the determining set of $u^{\mathrm{W}_{\mu}}$ on $L^{0}$.

Cherny [20] proves that, for any $X \in L^{0}, u^{\mathrm{W}_{\mu}}(X)$ can be represented by

$$
u^{\mathrm{W}_{\mu}}(X)=\int_{[0,1]} u^{\mathrm{ES}_{\lambda}}(X) \mu(d \lambda)=\int_{[0,1]} u^{\mathrm{ES}_{\lambda}}(X)^{+} \mu(d \lambda)-\int_{[0,1]} u^{\mathrm{ES}_{\lambda}}(X)^{-} \mu(d \lambda)
$$

with the convention $\infty-\infty=-\infty$.

In order to get a representation of $u^{\mathrm{W}_{\mu}}$ that is more useful for practical applications, we introduce the concept of distortion functions. A distortion function is an increasing concave function $\Psi:[0,1] \rightarrow[0,1]$. Let us state some important properties of distortion functions (see Föllmer and Schied [46]):

1. $\Psi$ is continuous on $(0,1]$ and admits a right-hand derivative. 
2. There is a one-to-one correspondence between a probability measure $\mu$ on $([0,1], \mathcal{B}([0,1]))$ and the distortion function $\Psi$ given by

$$
\Psi(y)=\mu(\{0\})+\int_{0}^{y} \int_{(t, 1]} s^{-1} \mu(d s) d t \quad \text { for every } y \in(0,1] .
$$

Henceforth, we write $\Psi_{\mu}$ instead of $\Psi$ for the distortion function when it is given by relation 2.4. As an example we consider the Dirac measure $\mu=\delta_{\lambda}$ with $\lambda \in[0,1]$ defined on $([0,1], \mathcal{B}([0,1]))$. Then, we obviously get $u^{\mathrm{W}_{\delta_{\lambda}}}=u^{\mathrm{ES}_{\lambda}}$. The corresponding distortion function is calculated from (2.4) as $\Psi_{\delta_{\lambda}}(y)=\frac{y}{\lambda} \wedge 1$ for any $\lambda \in(0,1]$. In the case where $\mu=\lambda_{[0,1]}$ is the Lebesgue measure restricted on $([0,1], \mathcal{B}([0,1]))$, we obtain

$$
\Psi_{\lambda_{[0,1]}}(y)= \begin{cases}-y \cdot \ln (y)+y, & y \in(0,1] \\ 0, & y=0\end{cases}
$$

In practical applications, the considered distortion functions are assumed to satisfy $\Psi_{\mu}(0)=0$ and $\Psi_{\mu}(1)=1$.

For every $X \in L^{0}$ and each probability measure $\mu$ on $([0,1], \mathcal{B}([0,1]))$ with corresponding distortion function $\Psi_{\mu}$, Cherny [20, Theorem 3.3] shows that $u^{W_{\mu}}$ can be written as a Lebesgue-Stieltjes integral of the form

$$
u^{W_{\mu}}(X)=\int_{\mathbb{R}} y \Psi_{\mu}\left(F_{X}(d y)\right)=-\int_{-\infty}^{0} \Psi_{\mu}\left(F_{X}(y)\right) d y+\int_{0}^{\infty} 1-\Psi_{\mu}\left(F_{X}(y)\right) d y,
$$

where $F_{X}$ denotes the cumulative distribution function of $X$ and we use the convention $\infty-\infty=-\infty$. The following diagram summarises the relationships between the quantities:

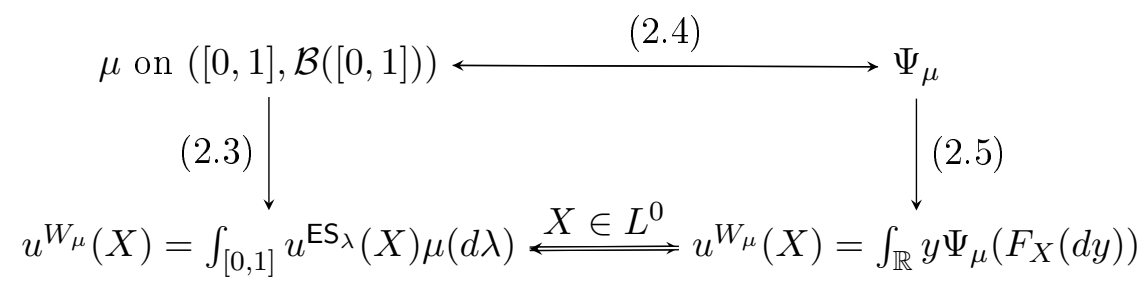

Hereafter, we consider a parameterised family $\left(u_{x}^{W}\right)_{x \geq 0}$ of Weighted Value at Risk utility functions. They are constructed through a specific family $\left(\Psi_{\mu_{x}}\right)_{x \geq 0}$ of distortion functions with corresponding family $\left(\mu_{x}\right)_{x \geq 0}$ of probability measures on $([0,1], \mathcal{B}([0,1]))$ by using representation 2.5. To simplify the notation, we set $\Psi_{x}:=\Psi_{\mu_{x}}$ for every $x \in[0, \infty)$. A family $\left(\Psi_{x}\right)_{x \geq 0}$ of distortion functions is called proper if for every $y \in(0,1)$ the map $x \mapsto \Psi_{x}(y)$ is increasing on $[0, \infty)$ and continuous on $(0, \infty)$.

Let $\left(\Psi_{x}\right)_{x \geq 0}$ be a proper family of distortion functions. Then, we obtain the decreasing 
family $\left(u_{x}^{W}\right)_{x \geq 0}$ of coherent utility functions on $L^{0}$ by setting

$$
\begin{aligned}
u_{x}^{W}(X):=u^{W_{\mu_{x}}}(X) & =\int_{\mathbb{R}} y \Psi_{x}\left(F_{X}(d y)\right) \\
& =-\int_{-\infty}^{0} \Psi_{x}\left(F_{X}(y)\right) d y+\int_{0}^{\infty} 1-\Psi_{x}\left(F_{X}(y)\right) d y .
\end{aligned}
$$

By decreasing, we mean that for every $X \in L^{0}$ it holds $u_{x_{2}}(X) \leq u_{x_{1}}(X)$ for all $x_{1} \leq x_{2}$.

We end this subsection with some crucial examples of proper families of distortion functions and their related families of utility functions. They are considered by Cherny and Madan [21, 22].

1. The family $\left(\Psi_{x}^{\mathrm{mv}}\right)_{x \geq 0}$ of MINVAR distortion functions is defined by

$$
\Psi_{x}^{\mathrm{mv}}:\left\{\begin{array}{l}
{[0,1] \rightarrow[0,1]} \\
y \mapsto 1-(1-y)^{1+x}
\end{array}\right.
$$

We plot some graphs of this function for different parameters in figure 2.1.

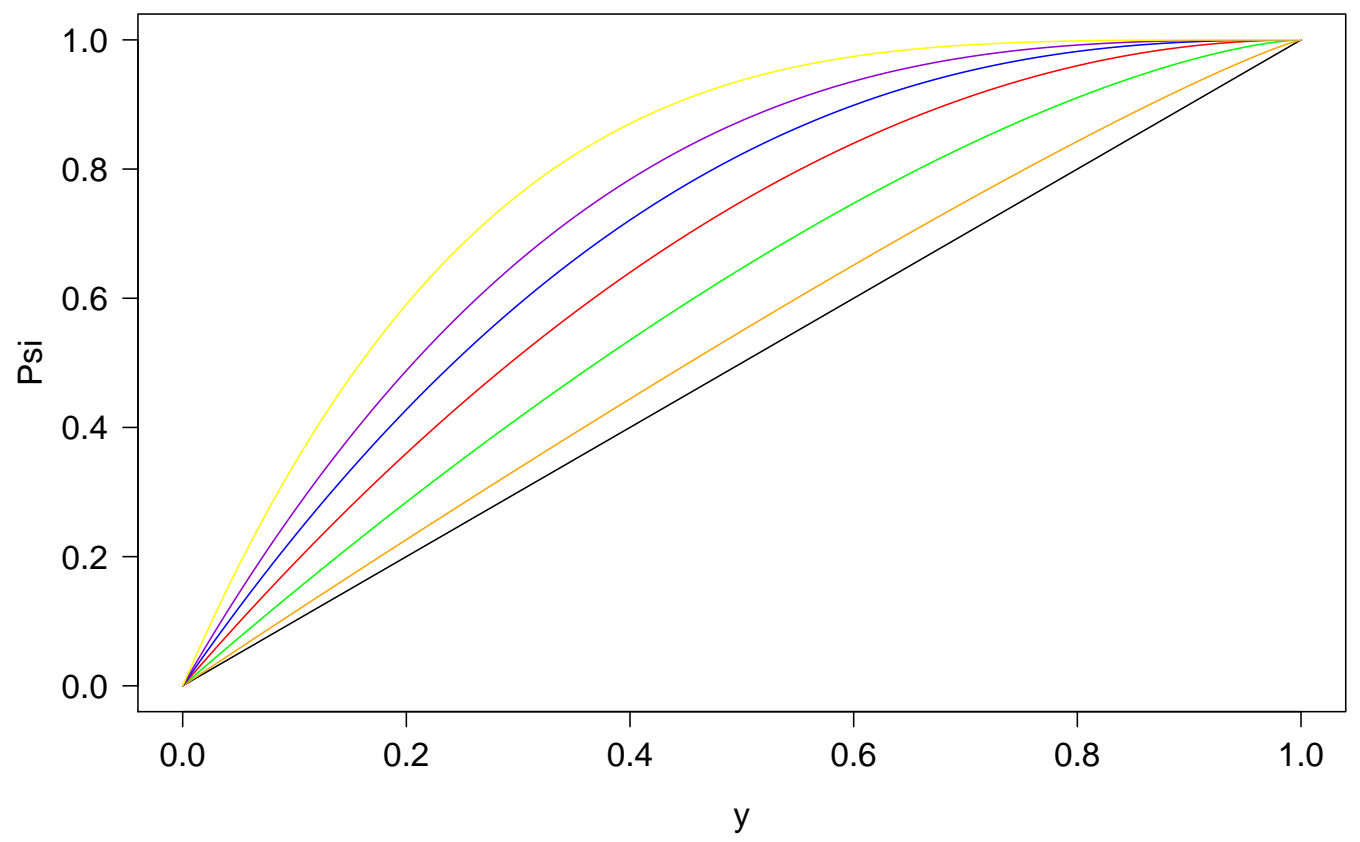

Figure 2.1.: Graphs of distortion function $\Psi_{\gamma}^{\mathrm{mv}}$ with different parameters: $(\bullet) \gamma=0,(\bullet)$ $\gamma=0.15,(\bullet) \gamma=0.5,(\bullet) \gamma=1,(\bullet) \gamma=1.5,(\bullet) \gamma=2$ and $(\bullet) \gamma=3$. 
2. The family $\left(\Psi_{x}^{\text {mav }}\right)_{x \geq 0}$ of MAXVAR distortion functions is defined by

$$
\Psi_{x}^{\operatorname{mav}}:\left\{\begin{array}{l}
{[0,1] \rightarrow[0,1]} \\
y \mapsto y^{\frac{1}{1+x}} .
\end{array}\right.
$$

It is represented in figure 2.2 .

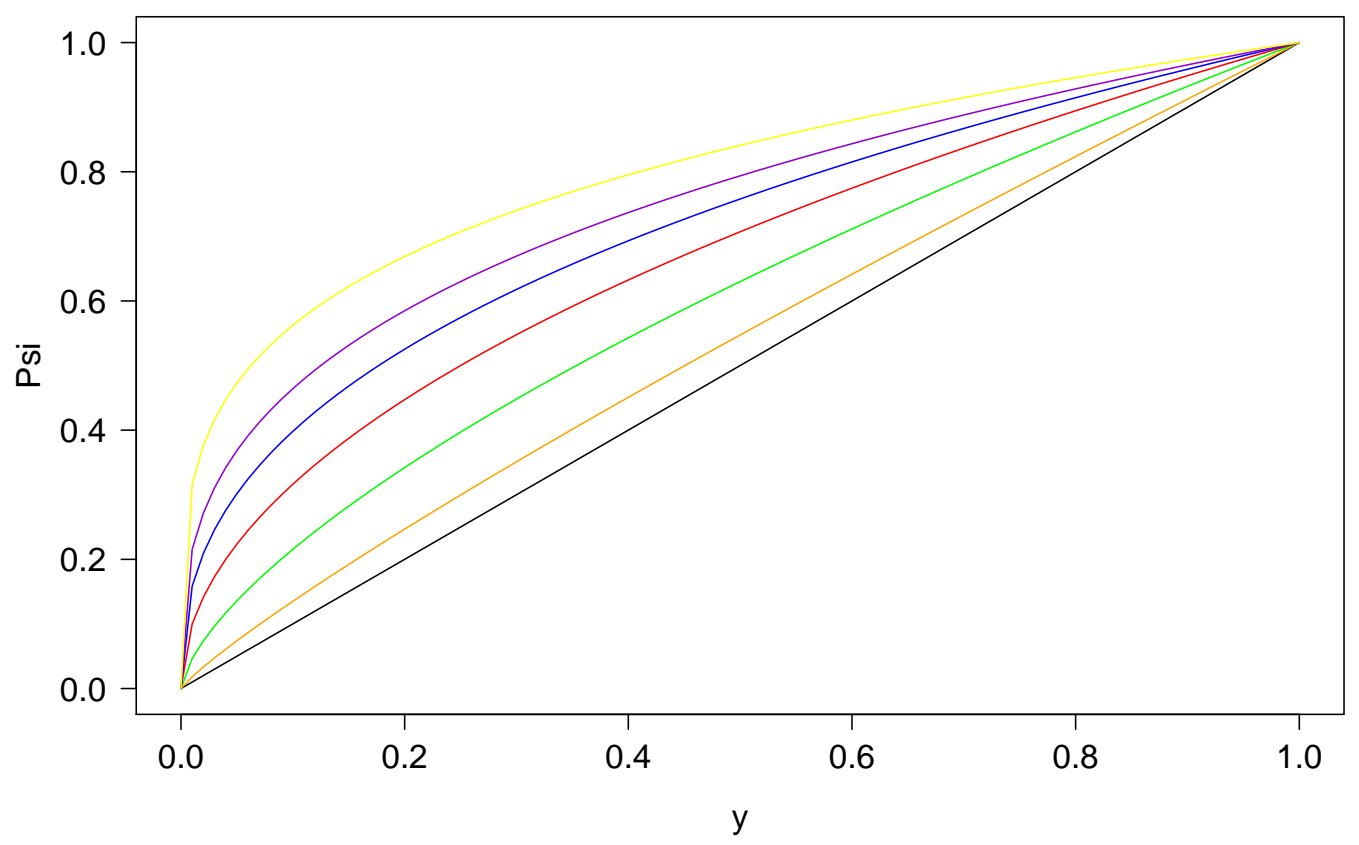

Figure 2.2.: Graphs of distortion function $\Psi_{\gamma}^{\text {mav }}$ with different parameters: $(\bullet) \gamma=0,(\bullet)$ $\gamma=0.15,(\bullet) \gamma=0.5,(\bullet) \gamma=1,(\bullet) \gamma=1.5,(\bullet) \gamma=2$ and $(\bullet) \gamma=3$.

3. The family $\left(\Psi_{x}^{\mathrm{mmv}}\right)_{x \geq 0}$ of MINMAXVAR distortion functions is determined by

$$
\Psi_{x}^{\mathrm{mmv}}:\left\{\begin{array}{l}
{[0,1] \rightarrow[0,1]} \\
y \mapsto 1-\left(1-y^{\frac{1}{1+x}}\right)^{1+x} .
\end{array}\right.
$$

In figure 2.3 we illustrate some graphs of $\Psi_{\gamma}^{\mathrm{mmv}}$ for different values of $\gamma \geq 0$.

4. The family $\left(\Psi_{x}^{\text {mamv }}\right)_{x \geq 0}$ of MAXMINVAR distortion functions is defined by

$$
\Psi_{x}^{\operatorname{mamv}}:\left\{\begin{array}{l}
{[0,1] \rightarrow[0,1]} \\
y \mapsto\left(1-(1-y)^{1+x}\right)^{\frac{1}{1+x}} .
\end{array}\right.
$$




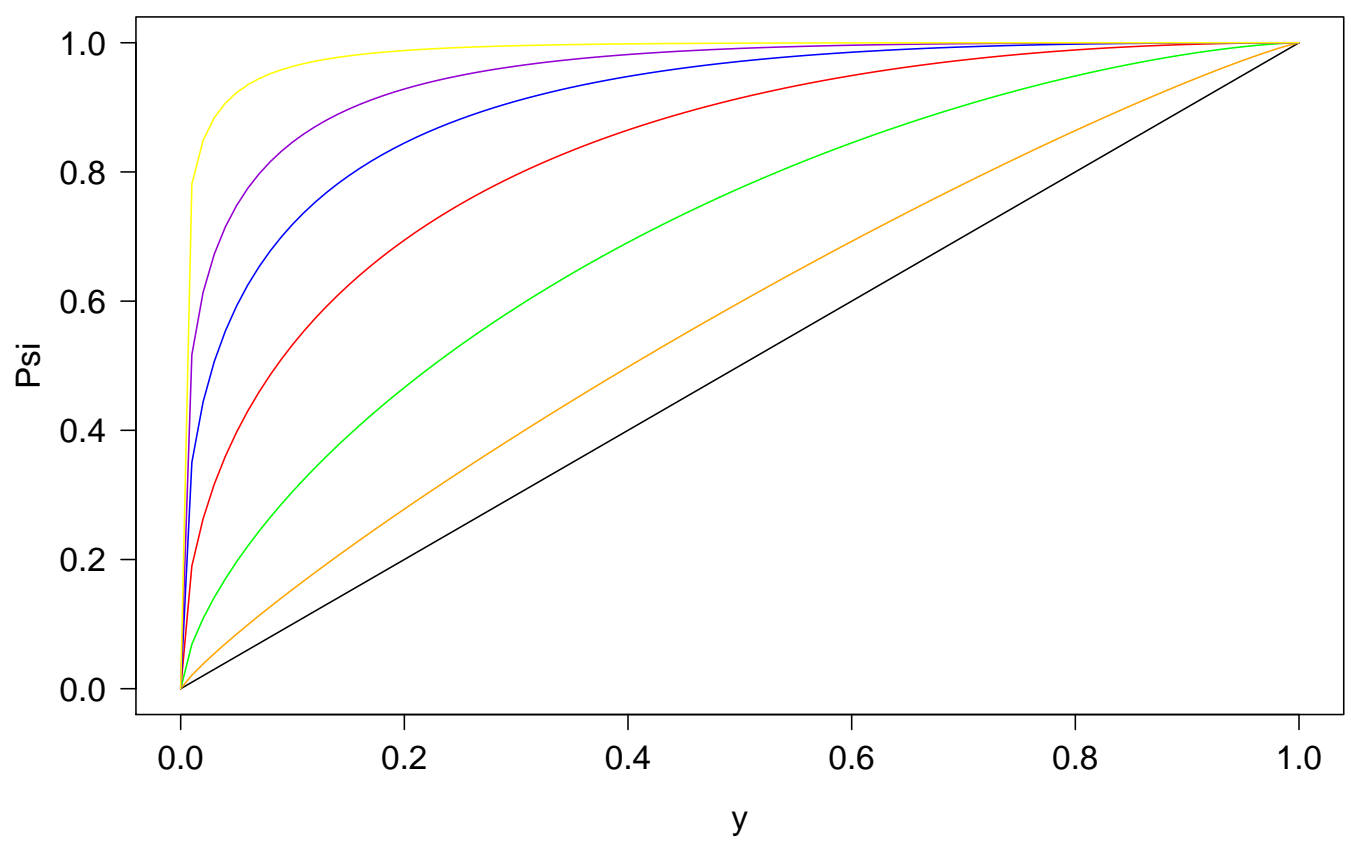

Figure 2.3: Graphs of distortion function $\Psi_{\gamma}^{\mathrm{mmv}}$ with different parameters: $(\bullet) \gamma=0$, $(\bullet) \gamma=0.15,(\bullet) \gamma=0.5,(\bullet) \gamma=1,(\bullet) \gamma=1.5,(\bullet) \gamma=2$ and $(\bullet) \gamma=3$.

Figure 2.4 presents some graphs of $\Psi_{\gamma}^{\text {mamv }}$ for different values of $\gamma \geq 0$.

The corresponding families of utility functions $\left(u_{x}^{\mathrm{mv}}\right)_{x \geq 0},\left(u_{x}^{\mathrm{mav}}\right)_{x \geq 0},\left(u_{x}^{\mathrm{mmv}}\right)_{x \geq 0}$ and $\left(u_{x}^{\text {mamv }}\right)_{x \geq 0}$ are defined by relation $(2.6)$.

\subsubsection{Acceptability Indices}

The reason why we consider a parameterised family of coherent utility functions is that the acceptance set of an investor is not constant over time. For instance, in boom times it is wide and in times of crisis it contracts.

Formally, let $\left(u_{x}\right)_{x \geq 0}$ be a decreasing family of coherent utility functions and $\left(\mathcal{A}_{u_{x}}\right)_{x \geq 0}$ be its corresponding family of acceptance cones. The parameter $x$ indicates the current stress level in the market. Note that for an increasing stress level we therefore get a contracting acceptable cone. Hereafter, a financial position with discounted payoff $X$ is called acceptable at level $x \geq 0$ if and only if $X \in \mathcal{A}_{u_{x}}$, i.e. we have $u_{x}(X) \geq 0$.

The concept of acceptability indices was introduced by Cherny and Madan [21]. An acceptability index associates its level of acceptability with each financial position. To be specific, an acceptability index $\alpha$ is a map $\alpha: L^{\infty} \rightarrow[0, \infty]$ satisfying the following 


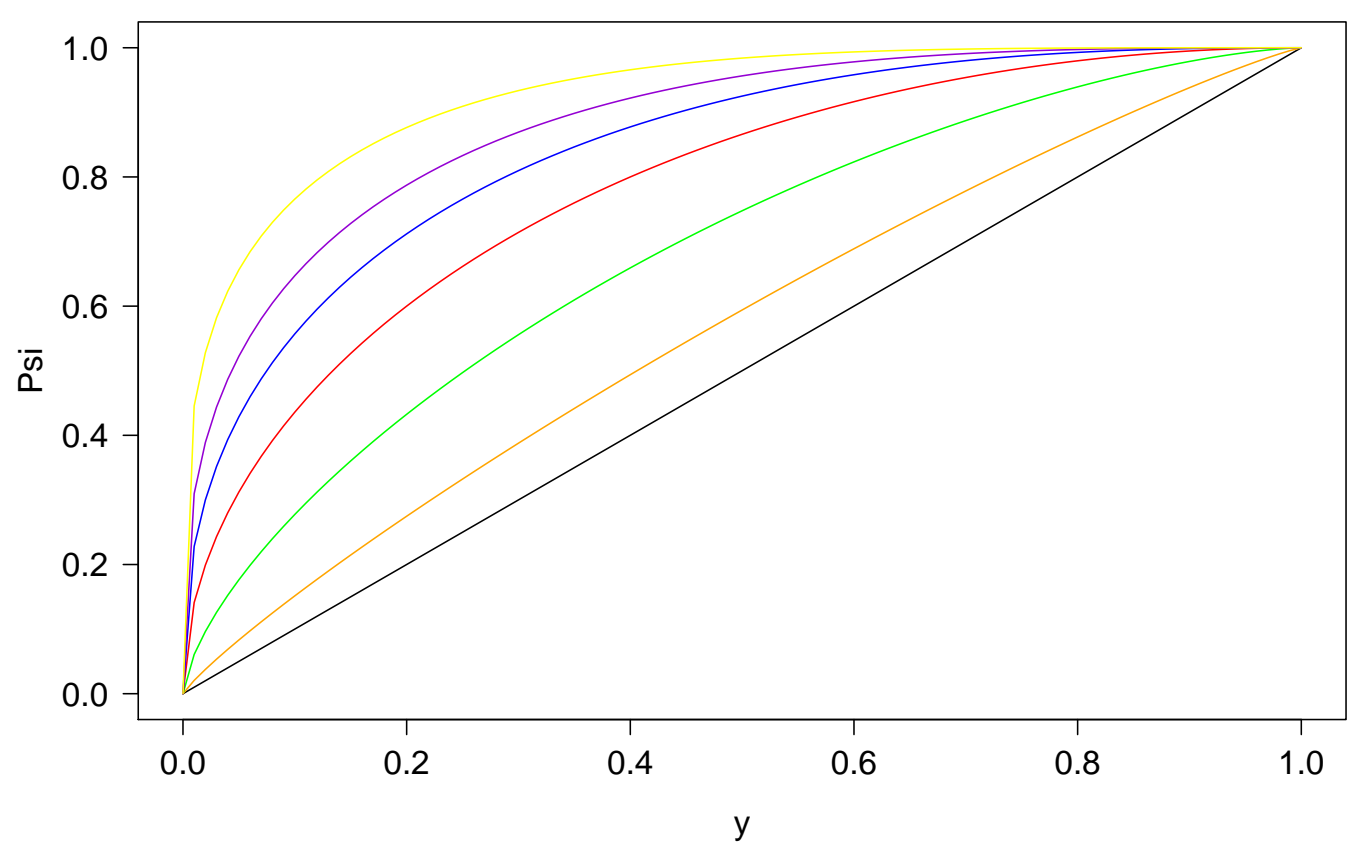

Figure 2.4.: Graphs of distortion function $\Psi_{\gamma}^{\text {mamv }}$ with different parameters: $(\bullet) \gamma=0$, $(\bullet) \gamma=0.15,(\bullet) \gamma=0.5,(\bullet) \gamma=1,(\bullet) \gamma=1.5,(\bullet) \gamma=2$ and $(\bullet) \gamma=3$.

properties (see Cherny and Madan [21]):

1. Monotonicity: If $X \leq Y$ a.s., then $\alpha(X) \leq \alpha(Y)$.

2. Quasi-concavity: If $x \leq \alpha(X)$ and $x \leq \alpha(Y)$, then $x \leq \alpha(\lambda X+(1-\lambda) Y)$ for every $\lambda \in[0,1]$.

3. Scale invariance: $\alpha(\lambda X)=\alpha(X)$ for any $\lambda>0$.

4. Fatou property: For a sequence of random variables $\left(X_{n}\right)$ satisfying $\left|X_{n}\right| \leq 1$, $x \leq \alpha\left(X_{n}\right)$ and $X_{n} \stackrel{P}{\rightarrow} X$, we have $x \leq \alpha(X)$.

An acceptability index can be represented by a decreasing family $\left(u_{x}\right)_{x \geq 0}$ of coherent utility functions on $L^{\infty}$ having the Fatou property or by an increasing family $\left(\mathcal{D}_{x}\right)_{x \geq 0}$ of subsets of $\mathcal{M}_{1}(P)$ (see Cherny and Madan [21]): a map $\alpha: L^{\infty} \rightarrow[0, \infty]$ is an acceptability index if and only if it has the form

$$
\alpha(X)=\sup \left\{x \geq 0 \mid u_{x}(X) \geq 0\right\}=\sup \left\{x \geq 0 \mid \inf _{Q \in \mathcal{D}_{x}} \mathbb{E}_{Q}[X] \geq 0\right\},
$$




\section{Two-Price Theory in Multiple-Curve Term Structure Models}

where we set $\inf \emptyset=\sup \emptyset=0$. As in the case of coherent utility functions, this representation is used to extend acceptability indices from space $L^{\infty}$ to $L^{0}$. More specifically, a map $\alpha: L^{0} \rightarrow[0, \infty]$ is called acceptability index on $L^{0}$ if it has a form $(2.8)$ with a decreasing family $\left(u_{x}\right)_{x \geq 0}$ of coherent utility functions on $L^{0}$ (or increasing family $\left(\mathcal{D}_{x}\right)_{x \geq 0}$, respectively). We say that $\alpha$ is represented by $\left(u_{x}\right)_{x \geq 0}$ (or $\left(\mathcal{D}_{x}\right)_{x \geq 0}$, respectively). By using the properties of a coherent utility function, one shows that this extension is consistent with the definition of $\alpha$ on $L^{\infty}$.

Let $\alpha$ be an acceptability index on $L^{0}$ that is represented by $\left(u_{x}\right)_{x \geq 0}$. Then, for every $X \in L^{0}$ and $x \geq 0$, we clearly have

$$
u_{x}(X) \geq 0 \Longrightarrow \alpha(X) \geq x .
$$

In general, the converse does not hold true. We call a random variable $X$ regular with respect to $\alpha$ if for every $x \geq 0$ we have

$$
u_{x}(X) \geq 0 \Longleftrightarrow \alpha(X) \geq x .
$$

Observe that, for every $X \in L_{w}^{1}(\alpha):=\bigcap_{x \geq 0} L_{w}^{1}\left(u_{x}\right)$ (referred to as the weak $L^{1}$-space of $\alpha$ ) satisfying $u_{0}(X)=\inf _{Q \in \mathcal{D}_{0}} \mathbb{E}_{Q}[X] \geq 0$, the random variable $X$ is regular with respect to $\alpha$ if, for every $x>0$, it holds

$$
\lim _{y \nearrow x} u_{y}(X)=u_{x}(X)
$$

\subsubsection{Weighted Value at Risk Acceptability Index}

Let $\left(u_{x}^{W}\right)_{x \geq 0}$ be a decreasing family of coherent utility functions constructed by a proper family $\left(\Psi_{x}\right)_{x \geq 0}$ of distortion functions with corresponding family $\left(\mu_{x}\right)_{x \geq 0}$ of probability measures on $([0,1], \mathcal{B}([0,1]))$ (see subsection 2.1.2). Then, the Weighted Value at Risk acceptability index $\alpha^{W}$ is defined by

$$
\alpha^{W}:\left\{\begin{array}{l}
L^{0} \rightarrow[0, \infty] \\
X \mapsto \sup \left\{x \geq 0 \mid u_{x}^{W}(X) \geq 0\right\} .
\end{array}\right.
$$

This approach leads to acceptability indices $\alpha^{\mathrm{mv}}, \alpha^{\mathrm{mav}}, \alpha^{\mathrm{mmv}}$ and $\alpha^{\text {mamv }}$ based on the families $\left(u_{x}^{\mathrm{mv}}\right)_{x \geq 0},\left(u_{x}^{\mathrm{mav}}\right)_{x \geq 0},\left(u_{x}^{\mathrm{mmv}}\right)_{x \geq 0}$ and $\left(u_{x}^{\mathrm{mamv}}\right)_{x \geq 0}$ introduced in subsection 2.1.2.

Proposition 2.1.1 Any $X \in L_{w}^{1}\left(\alpha^{W}\right)$ with $u_{0}^{W}(X) \geq 0$ is regular with respect to $\alpha^{W}$, i.e. for every $x \geq 0$, it holds

$$
\int_{\mathbb{R}} y \Psi_{x}\left(F_{X}(d y)\right) \geq 0 \Longleftrightarrow u_{x}^{W}(X) \geq 0 \Longleftrightarrow \alpha^{W}(X) \geq x .
$$


Proof: We only need to check that condition $(2.9)$ is satisfied. Then, the statement follows immediately (see the statements at the end of the last subsection). For any $X \in L_{w}^{1}\left(\alpha^{W}\right)$, we have $\left|u_{x}^{W}(X)\right|<\infty$ for every $x \geq 0$. Let $x$ and $s$ be chosen such that $0 \leq s<x$. Then, we have

$$
\left|u_{s}^{W}(X)-u_{x}^{W}(X)\right|=u_{s}^{W}(X)-u_{x}^{W}(X)=\int_{\mathbb{R}} \Psi_{x}\left(F_{X}(y)\right)-\Psi_{s}\left(F_{X}(y)\right) d y .
$$

Since the family of distortion functions is proper together with Elstrodt [43, Satz IV.5.6], it follows that the right-hand side converges to zero as $s \nearrow x$.

Note that in the situation of this Proposition, for any $a, b \in \mathbb{R}$ with $u_{0}^{W}(a X+b) \geq 0$, it holds that

$$
u_{x}^{W}(a X+b) \geq 0 \Longleftrightarrow \alpha^{W}(a X+b) \geq x
$$

for every $x \geq 0$.

We conclude from representation (2.7) and an application of the change-of-variable formula (cf. Billingsley [13, Theorem 16.13.]) that if

$$
\int_{-\infty}^{0} \Psi_{x}\left(F_{X}(y)\right) d y<\infty \text { and } \int_{0}^{\infty} \Psi_{x}\left(1-F_{X}(y)\right) d y<\infty
$$

for some $x \geq 0$, it holds $X \in L_{w}^{1}\left(u_{x}^{W}\right)$. Hence, a random variable $X$ satisfying $u_{0}^{W}(X) \geq 0$ is regular with respect to $\alpha^{W}$ on $[0, b) \subset \mathbb{R}_{+}\left(b \in \mathbb{R}_{+} \cup\{\infty\}\right)$ if condition 2.10 is valid for every $x \in[0, b)$.

A random variable $X$ has exponential moments in a neighbourhood of zero if there exists a constant $M>0$ such that we have $M_{X}(u)=\mathbb{E}[\exp (u X)]<\infty$ for every $u \in$ $[-M, M]$. A distribution $P_{X}$ of such an $X$ is also called light-tailed. Accordingly, one defines left-light-tailed $(u \in[-M, 0])$ and right-light-tailed $(u \in[0, M])$ distributions (for a detailed discussion see Foss, Korshunov, and Zachary [48]).

Lemma 2.1.1 Let $P_{X}$ be a light-tailed distribution with cumulative distribution function $F_{X}$ and constant $M$. Then, there exist constants $u_{1}, u_{2}, C_{1}$ and $C_{2}$ with $0<u_{1}, u_{2} \leq M$ and $C_{1}, C_{2}>0$ such that for every $y \in \mathbb{R}$, we have

$$
F_{X}(y) \leq C_{1} e^{u_{1} y}
$$

and

$$
1-F_{X}(y) \leq C_{2} e^{-u_{2} y} .
$$


Proof: We easily get

$$
F_{X}(y)=\int_{-\infty}^{y} P_{X}(d x)=e^{u_{1} y} \int_{-\infty}^{y} e^{-u_{1} y} P_{X}(d x) \leq e^{u_{1} y} M_{X}\left(-u_{1}\right)
$$

and

$$
1-F_{X}(y)=\int_{y}^{\infty} P_{X}(d x)=e^{-u_{2} y} \int_{y}^{\infty} e^{u_{2} y} P_{X}(d x) \leq e^{-u_{2} y} M_{X}\left(u_{2}\right) .
$$

Proposition 2.1.2 Suppose the random variable $X \in L^{0}$ has exponential moments in a neighbourhood of zero. Then, condition (2.10) is fulfilled for each $\Psi_{\gamma} \in\left\{\Psi_{\gamma}^{\mathrm{mv}}, \Psi_{\gamma}^{\mathrm{mav}}, \Psi_{\gamma}^{\mathrm{mmv}}, \Psi_{\gamma}^{\mathrm{mamv}}\right\}$ and $\gamma \geq 0$.

Proof: Let $\gamma \geq 0$ be fixed. For distortion function $\Psi_{\gamma}^{\text {mav }}$ the statement is obvious. To prove the claim for the other functions, let $d_{1}<0$ and $d_{2}>0$ be given and set

$$
c_{1}:=\frac{-\ln \left(C_{1}\right)}{u_{1}} \wedge d_{1}<0 \quad c_{2}:=\frac{\ln \left(C_{2}\right)}{u_{2}} \vee d_{2}>0
$$

where the constants $C_{1}, C_{2}, u_{1}$ and $u_{2}$ are as in Lemma 2.1.1. An easy analysis of a convex function shows that

$$
(1+x)^{r} \geq 1+r x
$$

for every $x>-1$ and $r \geq 1$ (Bernoulli's inequality). We obtain for the distortion function $\Psi_{\gamma}^{\mathrm{mmv}}$ that

$$
\begin{aligned}
\int_{-\infty}^{0} \Psi_{\gamma}^{\mathrm{mmv}}\left(F_{X}(y)\right) d y & \leq \int_{-\infty}^{0} \Psi_{\gamma}^{\mathrm{mmv}}\left(C_{1} e^{u_{1} y}\right) d y \\
& \leq K_{1}+\int_{-\infty}^{c_{1}} 1-\left(1-C_{1}^{\frac{1}{1+\gamma}} e^{\frac{u_{1}}{1+\gamma} y}\right)^{1+\gamma} d y \\
& \leq K_{1}+\int_{-\infty}^{c_{1}} 1-\left(1+(1+\gamma)\left(-C_{1}^{\frac{1}{1+\gamma}} e^{\frac{u_{1}}{1+\gamma} y}\right)\right) d y \\
& =K_{1}+(1+\gamma) C_{1}^{\frac{1}{1+\gamma}} \int_{-\infty}^{c_{1}} e^{\frac{u_{1}}{1+\gamma} y} d y<\infty
\end{aligned}
$$


and

$$
\begin{aligned}
\int_{0}^{\infty} \Psi_{\gamma}^{\mathrm{mmv}}\left(1-F_{X}(y)\right) d y & \leq \int_{0}^{\infty} \Psi_{\gamma}^{\mathrm{mmv}}\left(C_{2} e^{-u_{2} y}\right) d y \\
& \leq K_{2}+\int_{c_{2}}^{\infty} 1-\left(1-C_{2}^{\frac{1}{1+\gamma}} e^{-\frac{u_{2}}{1+\gamma} y}\right)^{1+\gamma} d y \\
& \leq K_{2}+\int_{c_{2}}^{\infty} 1-\left(1+(1+\gamma)\left(-C_{2}^{\frac{1}{1+\gamma}} e^{-\frac{u_{2}}{1+\gamma} y}\right) d y\right. \\
& =K_{2}+(1+\gamma) C_{2}^{\frac{1}{1+\gamma}} \int_{c_{2}}^{\infty} e^{-\frac{u_{2}}{1+\gamma} y} d y<\infty
\end{aligned}
$$

with $K_{1}, K_{2}>0$. The verification for the remaining functions can be shown analogously.

\subsubsection{Ask and Bid Prices}

The theory of two prices (ask and bid prices) based on the concept of acceptability indices was developed by Cherny and Madan [22] and is referred to as conic finance. In this context, we also mention the work of Eberlein and Madan [35] and Eberlein [30].

Recall that the market appears as a passive counterparty that decides to accept or not accept a quantity. The price for buying a product from the market is called ask price and the price for selling it to the market is referred to as bid price.

Let $\alpha$ be an acceptability index represented by a decreasing family $\left(u_{x}\right)_{x \geq 0}$ of coherent utility functions and $\gamma \geq 0$ be a fixed level of acceptability. We define by

$$
\mathcal{A}_{\gamma}(\alpha):=\left\{X \in L^{0} \mid \alpha(X) \geq \gamma\right\}
$$

the set of acceptable discounted payoffs at level $\gamma$. The parameter $\gamma$ can be seen as the current level of market (il)liquidity. Furthermore, consider a regular discounted payoff $X \in L^{0}$ with respect to $\alpha$. Motivated by market competition, the ask price $a_{\gamma}(X)$ of $X$ at level $\gamma$ with respect to $\alpha$ is the smallest number $a$ such that $a-X$ is accepted by the market. Consequently, we define

$$
a_{\gamma}(X):=\inf \{a \in \mathbb{R} \mid \alpha(a-X) \geq \gamma\} \text {. }
$$

We easily obtain the non-linear expression

$$
\begin{aligned}
a_{\gamma}(X) & =\inf \left\{a \in \mathbb{R} \mid u_{\gamma}(a-X) \geq 0\right\}=\inf \left\{a \in \mathbb{R} \mid \inf _{Q \in \mathcal{D}_{\gamma}} \mathbb{E}_{Q}[a-X] \geq 0\right\} \\
& =\inf \left\{a \in \mathbb{R} \mid a+\inf _{Q \in \mathcal{D}_{\gamma}} \mathbb{E}_{Q}[-X] \geq 0\right\}=-\inf _{Q \in \mathcal{D}_{\gamma}} \mathbb{E}_{Q}[-X] \\
& =\sup _{Q \in \mathcal{D}_{\gamma}} \mathbb{E}_{Q}[X] .
\end{aligned}
$$




\section{Two-Price Theory in Multiple-Curve Term Structure Models}

Analogously, we define the bid price $b_{\gamma}(X)$ of $X$ at level $\gamma$ with respect to $\alpha$ as the largest number $b$ such that $X-b$ is accepted. Therefore, we have

$$
b_{\gamma}(X):=\sup \{b \in \mathbb{R} \mid \alpha(X-b) \geq \gamma\} .
$$

One immediately gets the non-linear formula

$$
b_{\gamma}(X)=\inf _{Q \in \mathcal{D}_{\gamma}} \mathbb{E}_{Q}[X]
$$

Observe that we naturally have $b_{\gamma}(X) \leq a_{\gamma}(X)$.

As a particularly important case, we consider ask and bid prices based on Weighted Value at Risk acceptability indices. In this situation, we obtain the useful representations for the ask price

$$
\begin{aligned}
a_{\gamma}(X) & =\inf \left\{a \in \mathbb{R} \mid u_{\gamma}^{W}(a-X) \geq 0\right\}=-u_{\gamma}^{W}(-X) \\
& =-\int_{\mathbb{R}} y \Psi_{\gamma}\left(F_{-X}(d y)\right)=\int_{-\infty}^{0} \Psi_{\gamma}\left(F_{-X}(y)\right) d y-\int_{0}^{\infty} 1-\Psi_{\gamma}\left(F_{-X}(y)\right) d y \\
& =\int_{0}^{\infty} \Psi_{\gamma}\left(1-F_{X}(y)\right) d y-\int_{-\infty}^{0} 1-\Psi_{\gamma}\left(1-F_{X}(y)\right) d y
\end{aligned}
$$

and the bid price

$$
\begin{aligned}
b_{\gamma}(X) & =\sup \left\{b \in \mathbb{R} \mid u_{\gamma}^{W}(X-b) \geq 0\right\}=u_{\gamma}^{W}(X) \\
& =\int_{\mathbb{R}} y \Psi_{\gamma}\left(F_{X}(d y)\right)=-\int_{-\infty}^{0} \Psi_{\gamma}\left(F_{X}(y)\right) d y+\int_{0}^{\infty} 1-\Psi_{\gamma}\left(F_{X}(y)\right) d y .
\end{aligned}
$$

Observe that the ask and bid prices depend on an underlying probability measure. In our situation, this will be a basic pricing measure which is distorted by the family $\Psi$ to obtain the ask and bid prices (it is assumed to be contained in the set $\mathcal{D}_{\gamma}$ ). These facts are crucial when we apply the two-price theory to the multiple-curve term structure model (see section 2.3).

\subsection{Multiple-Curve Interest Rate Term Structure Modelling}

In this section, we present the multiple-curve interest rate modelling approach. First, we briefly recall the fundamental model framework and relevant interest rate derivatives. Then, we address the modern multiple-curve setting. 


\subsubsection{Fundamental Model Approaches}

In equity markets, it is a priori clear which quantity is fundamental and has to be modelled, namely the stock price. In contrast, fixed income markets provide some freedom to choose which quantity is considered to be basic and which quantities are derived from this basic one. Concretely, the fundamental model quantities can be zero coupon bond prices, instantaneous forward rates, short rates, forward prices or (forward) reference rates (Libor/Euribor).

A default-free zero coupon bond is defined as a financial security that provides an amount of one currency unit to its owner at maturity without intermediate coupon payments. We denote by $B_{t}(T)$ the price of such a security at time $t \in[0, T]$ when it matures at time $T \in\left[0, T^{*}\right]$, where $T^{*}>0$ is a finite time horizon. One also refers to $B_{t}(T)$ as discount factor. The interest rate that applies for an instantaneous period of time is called short rate. The value of this rate at time $t$ is denoted by $r_{t}$. The instantaneous, continuously compounded forward rate is defined as the forward rate that applies for an infinitesimal period of time starting at some (future) specific date. More specifically, we call $f_{t}(T)$ the forward interest rate at time $t \leq T$ for instantaneous borrowing or lending at date $T$. Intuitively, we can interpret $f_{t}(T)$ as the interest rate over the infinitesimal period of time $[T, T+d T]$ considered from time $t$. That is why $f_{t}(T)$ is referred to as instantaneous, continuously compounded forward rate or shortly, instantaneous forward rate. The instantaneous forward rate is closely related to a zero coupon bond by the equation

$$
B_{t}(T)=\exp \left(-\int_{t}^{T} f_{t}(u) d u\right)
$$

Let us denote by $\frac{\partial}{\partial T}$ the differential operator with respect to variable $T$. From the last equation, $f_{t}(T)$ can be represented by

$$
f_{t}(T)=-\frac{\partial}{\partial T} \ln B_{t}(T)
$$

where we assume that the (partial) derivative exists. The instantaneous forward rate is related to the short rate by $r_{t}=f_{t}(t)$. Another important quantity is called the forward price. It is defined at time $t$ for the maturities $S$ and $T$ by the quotient of the corresponding zero coupon bond prices, namely

$$
F_{B}(t, S, T):=\frac{B_{t}(S)}{B_{t}(T)} .
$$

Let $t, S, T \in\left[0, T^{*}\right]$ satisfying $t \leq S \leq T$ and $\delta(S, T)$ denote the year fraction between $S$ and $T$ according to a specified day count convention (for details see Brigo and Mercurio [17, section I.1.2]). The time- $t \delta(S, T)$-forward reference rate $L_{t}(S, T)$ is the discretely 


\section{Two-Price Theory in Multiple-Curve Term Structure Models}

compounded annualised interest rate that can be earned for an interval starting at date $S$ and ending at $T$. It is defined by

$$
L_{t}(S, T):=\frac{1}{\delta(S, T)}\left(\frac{B_{t}(S)}{B_{t}(T)}-1\right) .
$$

This rate can be seen as the Libor or Euribor. Note that we define this quantity as risk-free rate here but we emphasise that credit and liquidity risk can also be taken into account in term structure models (see for instance Kluge [64] and Jarrow and Roch [58]).

To sum up, the following master equation which clarifies the relations between all relevant quantities is valid:

$$
1+\delta(S, T) L_{t}(S, T)=\frac{B_{t}(S)}{B_{t}(T)}=F_{B}(t, S, T) .
$$

Another fundamental difference between the equity and the fixed income market is the number of underlying securities one has to consider. In the former it is sufficient to model at most a finite collection of securities, whereas in the latter one typically considers a continuum. More precisely, we have one security for every maturity $T \in$ $\left[0, T^{*}\right]$. In our model, we focus on a finite number of securities since we only consider a finite set of maturities. To this end, let us fix a collection of reset and settlement dates $\mathscr{T}:=\left\{T_{0}, T_{1}, \ldots, T_{n}\right\}$, where we assume that $0 \leq T_{0}<T_{1}<\cdots<T_{n}$ with $n \in \mathbb{N}$ and $T_{n}=T^{*}$. The time between two consecutive reset dates is known as tenor and $\mathscr{T}$ is called discrete tenor structure. We define by $\delta_{k}:=\delta\left(T_{k-1}, T_{k}\right)$ the year fraction between the dates $T_{k-1}$ and $T_{k}$ for every $k \in\{1, \ldots, n\}$. Let us assume that the year fractions $\delta_{1}, \ldots, \delta_{n}$ between each pair of consecutive dates are equidistant and we may therefore set $\delta:=\delta_{k}$ for every $k \in\{1, \ldots, n\}$. Thus, the considered discrete tenor structure $\mathscr{T}$ is unambiguously related to tenor $\delta$. We call such a tenor structure equidistant. For each pair of dates from the discrete tenor structure, the master equation results in

$$
1+\delta L_{t}\left(T_{k-1}, T_{k}\right)=\frac{B_{t}\left(T_{k-1}\right)}{B_{t}\left(T_{k}\right)}=F_{B}\left(t, T_{k-1}, T_{k}\right)
$$

for every $k \in\{1, \ldots, n\}$.

We end this subsection by an overview about some important publications that are related to interest rate modelling: A substantial collection of short rate models driven by diffusion processes was developed by Merton and Vasicek. Brigo and Mercurio [17, part II] present a detailed and profound summary about this type of models. Especially, they devote the way from short rate models to the Heath-Jarrow-Morton (HJM) approach (see Heath, Jarrow, and Morton [52]) on which the (multiple-curve) Lévy forward rate model is based (see chapter 3). The Lévy forward rate model was developed in a series of papers: Eberlein and Raible [38, Eberlein and Özkan [37, Eberlein, Jacod, and 
Raible [39] and Eberlein and Kluge [33]. The basic model quantity is the instantaneous, continuously compounded forward rate driven by a (time-inhomogeneous) Lévy process. As opposed to the instantaneous forward rates that are a mathematical idealisation rather than an observable quantity, the (forward) reference rates are observable in the market (Libor or Euribor). Therefore, Brace, Gątarek, and Musiela [15] have chosen this rate as fundamental model quantity and introduced the Libor market model. As a generalisation of their approach, the Lévy Libor (market) model was introduced by Eberlein and Özkan [37. In this context, the Lévy forward price model was developed by the same authors.

\subsubsection{Interest Rate Derivatives}

We emphasise that an interest rate model should be able to reproduce the observable term structure of interest rates and the market prices of interest rate derivatives. Interest rate derivatives are financial products whose payoffs depend in some functional form on the value of the interest rate. The volume of trading in these derivatives both in the exchangetraded and the over-the-counter market has been rapidly increased in the last years. One of the key challenges for market participants is to develop suitable (mathematical) models that are used to evaluate these instruments. This task has become even more important since the beginning of the global financial crisis in 2007.

Subsequently, we describe some common interest rate derivatives that are considered in this work. A more detailed explanation of these contracts are given by Hull [55], Filipovic [44], Musiela and Rutkowski [75] and Brigo and Mercurio [17].

\section{Floating-Rate Note}

A floating-rate note (FRN) is a contract traded in the over-the-counter market that ensures the payment at a reference interest rate at some future dates. This financial product pays a last cash flow consisting of the notional principal at its maturity.

\section{Forward Rate Agreements}

A forward rate agreement (FRA) is an over-the-counter contract between two market participants. It is constructed to ensure that for one party a certain fixed interest rate will be valid to either lending or borrowing the notional principal to or from the other party during an agreed future period of time. The basic assumption of the contract is that the (lending or borrowing) payment would normally be done at a floating reference interest rate. Therefore, the (possible negative) amount that a party earns (or loses) depends on the difference between the fixed and the floating rate. The usual market practice for forward rate agreements is that all rates are measured with a compounding frequency according to the length of the considered future period of time (tenor). For instance, if we assume that the year fraction of this period is equal to $\delta=0.25$, then the 


\section{Two-Price Theory in Multiple-Curve Term Structure Models}

rates are expressed with quarterly compounding and the reference interest rate refers to the three-month tenor (for instance the three-month Libor). Another interpretation of a forward rate agreement is that both market participants agree to exchange payments at some future date, where one party receives interest on a principal at the arranged fixed rate and pays interest on the same principal at the realised floating reference rate. Accordingly, the other party pays interest on the principal at the fixed rate and receives interest on the principal at the floating rate.

\section{Interest Rate Swaps}

A swap is an over-the-counter contract between two individuals to exchange cash flows in the future. This contract precisely specifies the payment dates and the way in which the payments have to be determined. Swaps typically lead to cash flows that are exchanged between two market participants on several future dates (for instance, along a discrete tenor structure). The calculation of the cash flows is usually based on the future value of an interest rate, an exchange rate or another market variable. A forward swap is a swap agreement that starts at some future date.

A plain vanilla (forward) interest rate swap (IRS) is the most common type of a swap. Here an investor agrees to pay cash flows equal to interest at a predetermined fixed rate on a notional principal at some predetermined future dates to the other party. In return, the investor receives interest rate payments at a floating interest rate on the same notional principal from the other party. The payment stream indexed on the fixed rate is referred to as the fixed leg and the payment stream indexed on the floating rate is called the floating leg. The floating rate is usually tied to a reference rate such as the Libor or the Euribor. We consider interest rate swaps which are settled in arrears. This means that the floating rate is set at the beginning of a period of time and pays at the end of this period. One says that an interest rate swap is settled in advance if the payments are made at the beginning of each period of time. The fixing and payment dates corresponding to the respective legs are given by discrete tenor structures. The (mid-market) fixed rate that will be exchanged for the reference rate is known as (forward) swap rate. It is specified as the rate which sets the value of the contract equal to zero.

Another type of a swap is the basis swap (BS). Here, two payments that are each based on a separate floating reference rate are exchanged.

\section{Caps and Floors}

Interest rate caps and floors are actively traded interest rate options offered by financial institutions in the over-the-counter market. To explain these contracts we consider a floating-rate note in which a reference interest rate is fixed along a discrete tenor structure. As usual, we call the period of time between two consecutive reset dates tenor. An 
interest rate cap is designed to provide insurance against the possibility that the reference interest rate based on such a floating-rate note exceeds a certain level. This level is denoted as cap rate. At each fixing date up to the maturity of the cap the reference rate is observed. Either there is no payout if the rate is below the cap rate or there is a payout if the rate is above. The payout is the difference between the interest rate and the cap rate. A payout occurs at the end of the period and depends on the excess, tenor and notional principal. Hence, a cap can be characterised as a portfolio of call options with underlying reference rate. These call options are known as caplets. Interest rate floors are defined in an analogous way as caps. A floor provides a payout if the reference interest rate is below a certain rate (floor rate) at the reset date. The payment also occurs a tenor after the fixing date. A floor can therefore be regarded as a portfolio of put options on the reference rate. Each option is named floorlet.

\section{Digital Options}

A standard European interest rate digital call (put) with strike rate $B>0$ is a financial security which pays an amount of one unit of currency to its owner if and only if the simply compounded reference interest rate lies above (below) $B$ at its maturity $T_{1} \in \mathbb{R}_{+}$. An interest digital option is called delayed if the option maturity $T_{1}$ and the payment date $T$ differ in the sense of $T_{1} \leq T$. Note that a standard digital option can be seen as a special case of a delayed digital option by setting $T_{1}=T$. A delayed range digital option provides a terminal payoff equal to one paid at date $T$ if and only if the underlying reference rate lies inside a pre-specified corridor at maturity $T_{1}$.

\subsubsection{The Multiple-Curve Approach}

Before the global financial crisis started in the summer of 2007, interest rate quotes showed typical consistencies. These conditions were assumed to be valid and stated in literature as an indisputable fact. The consistencies between rates allowed to construct a unique well-defined term structure of interest rates (bond curve) by using standard bootstrapping techniques. Of course, differences between similar rates were present in the market but could be regarded as negligible in general. For instance, interest rate swaps that have the same maturity but are based on different discrete tenor structures quoted swap rates at a small but negligible spread before the crisis (see figure 2.13).

Then, the global financial crisis caused a number of anomalies in the fixed income market. As a result, the interest rates that were formerly consistent with each other started to diverge substantially. This issue led to a degree of incompatibility between them. Generally speaking, the substantial changes concerned market rates that are implicitly related to different tenors.

An example is the forward rate that is implied by the EONIA overnight indexed swap rate quote. This rate became a different object than the quoted FRA rate. This issue 


\section{Two-Price Theory in Multiple-Curve Term Structure Models}

is illustrated in figures 2.5 and 2.6. Another example is given by the divergence of the Euribor and the associated EONIA OIS rates presented in figures 2.7 2.11 The corresponding spreads between these rates are demonstrated in figure 2.12 This graph illustrates the monotonicity of the different curves which is considered in section 3.2 . The greater the underlying tenor, the greater the spread between Euribor and EONIA OIS rate. Another anomaly can be observed by the arising differences of the swap rates with respect to swaps with different payment frequencies (different discrete tenor structures). More specifically, a swap rate based on semiannual payments indexed by the six-month Euribor can be different than the swap rate based on quarterly payments indexed by the three-month Euribor if both swap contracts have the same maturity. This issue is illustrated in figure 2.13. To sum up, the tenor dependence of market rates reveals an additional significant influence factor in fixed income markets with beginning of the global financial crisis.

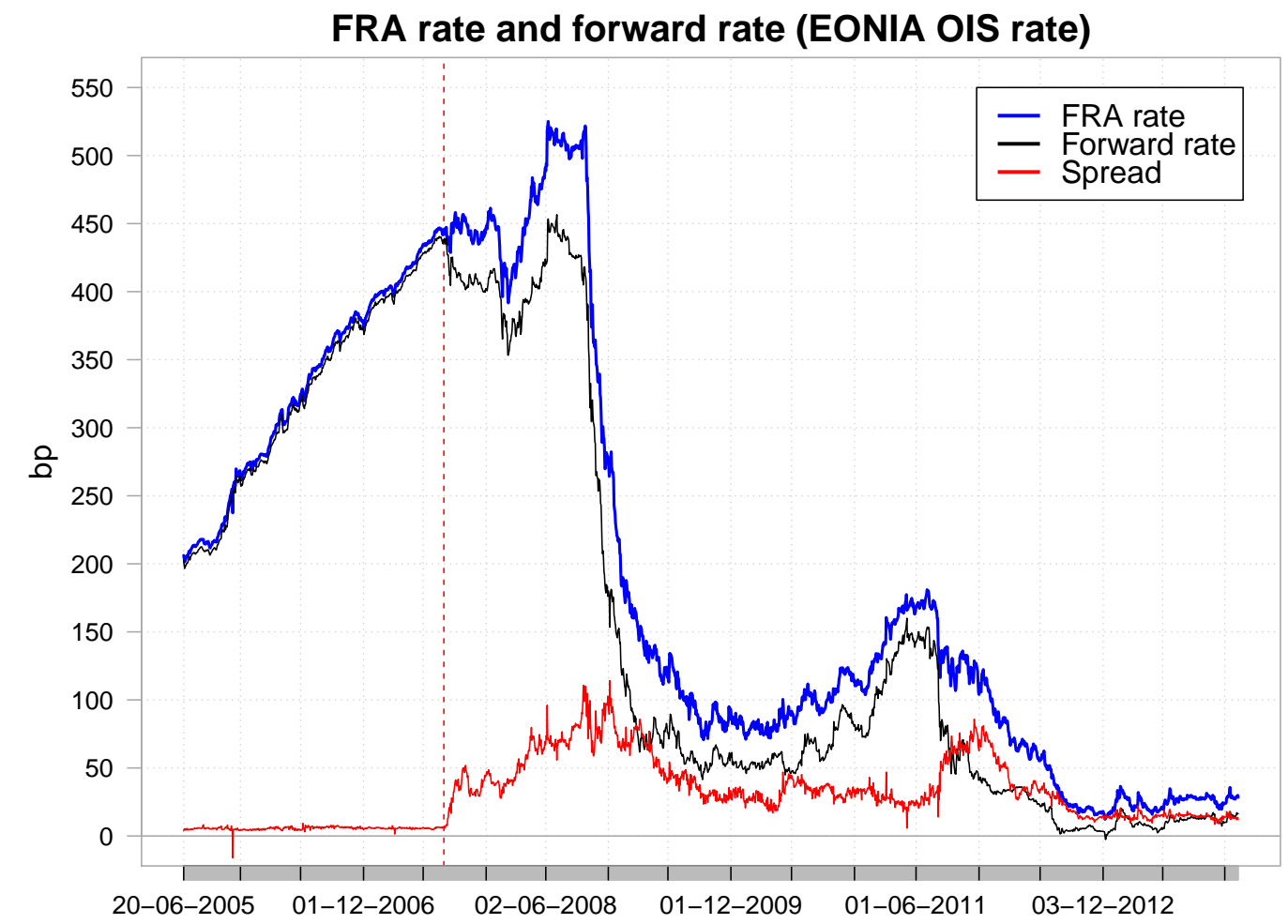

Figure 2.5.: Divergence of forward rates (based on EONIA OIS quote) and 3x6 FRA rates.

All these changes that occurred in the quotes of market rates can be explained by credit and liquidity risk. A nice treatment of the notion of credit risk is given by Schönbucher [82]. Generally speaking, credit risk can be defined as the risk that a debtor does not fulfil his payment obligations. Liquidity risk can be characterised by the following three 


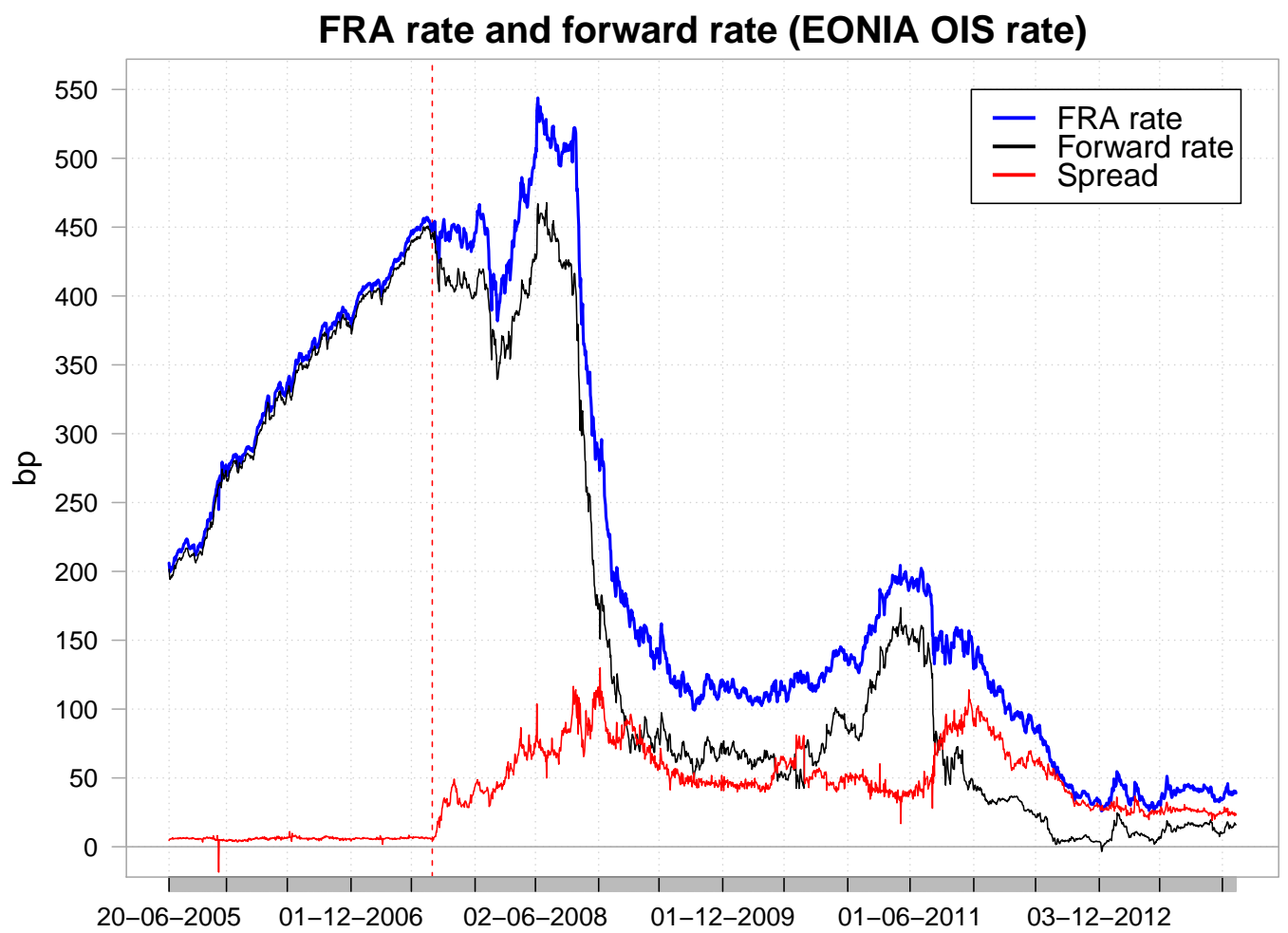

Figure 2.6.: Divergence of forward rates (based on EONIA OIS quote) and 3x9 FRA rates.

issues (see Acerbi and Scandolo [1, Brunnermeier and Pedersen [19] and Bianchetti and Carlicchi [11]):

1. Funding liquidity risk: The lack of liquidity to meet short term debt obligations.

2. Market liquidity risk: The risk of trading and holding positions in illiquid markets.

This fact is generally accompanied by an excessive spread of the ask and bid prices.

3. Systemic Liquidity Risk: The difficulty to borrow funds on the market due to funding cost.

Note that the liquidity risk component in reference interest rates is distinct but strongly correlated to the credit risk component. In this thesis, market liquidity risk is modelled by the two-price theory introduced in section 2.1. The other types of liquidity risk are also considered in form of a liquidity component in the drift function.

The influence of credit risk in the crisis is emphasised by figure 2.14, where the connection between the Euribor/EONIA OIS spread and CDS spreads of some panel banks are illustrated. Credit risk is obviously a reason for the market anomalies. The effect of liquidity risk is illustrated by Eberlein [30]. All these facts together imply that a 


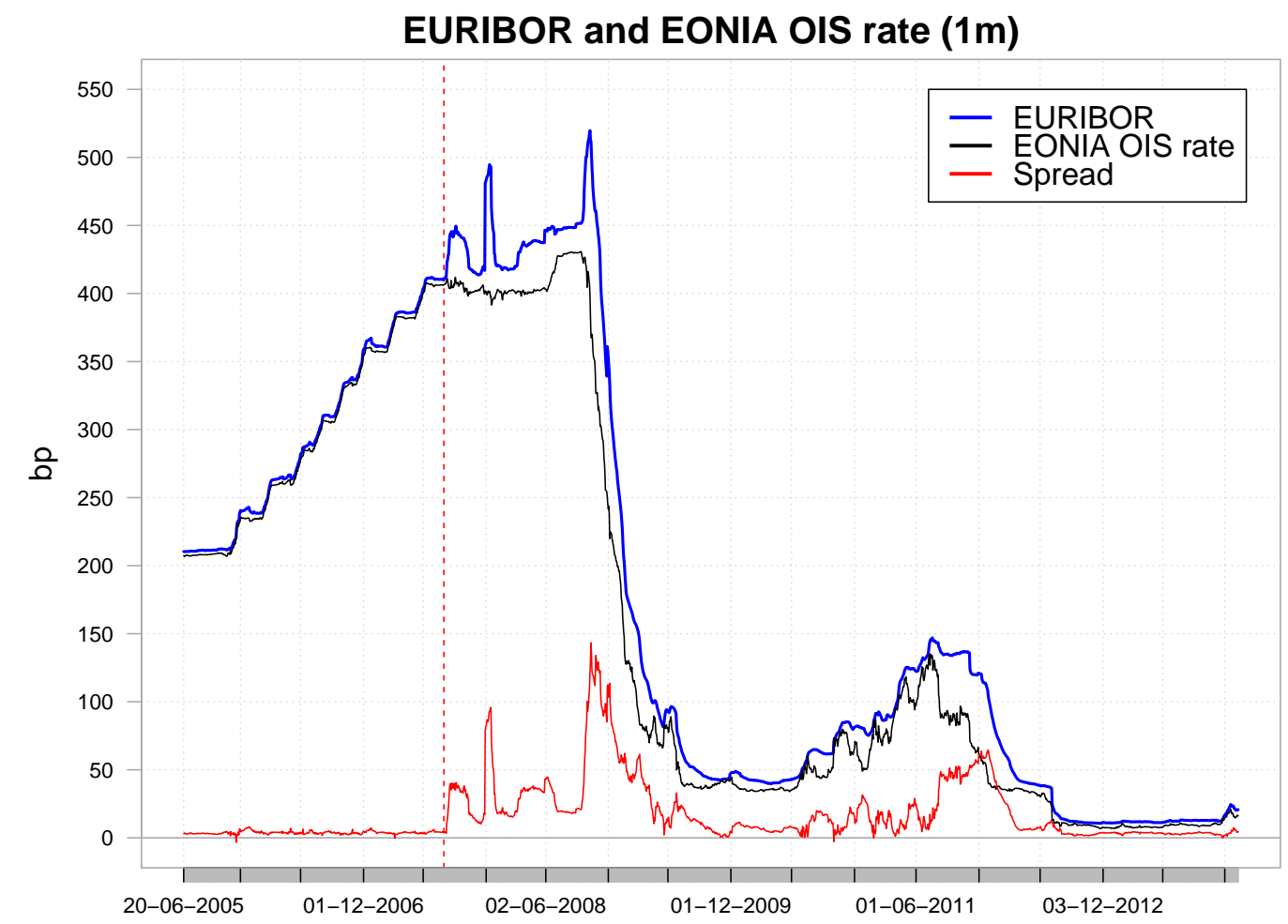

Figure 2.7.: Evolution of the EURIBOR and EONIA OIS rates corresponding to the one-month tenor.

consistent construction of a term structure of interest rates and the correct valuation of interest rate derivatives have to take into account credit and liquidity theories.

We stress that, by taking into consideration credit and liquidity risk, this new market environment does not necessarily generate arbitrage opportunities. Therefore, credit and liquidity models provide a theoretical justification for the current market situation. Since the development of a model that considers both types of risk is a challenging task, practitioners preferred using an empirical approach. They divide market rates into different risk classes depending on their respective tenor. This method results in the consideration of a discount curve and as many different term structure curves as occurring tenors. We call this procedure multiple-curve approach. It should be mentioned that the distinct curves in the market reflect the various magnitudes of credit and liquidity risk that are included in interest rates.

The appearance of negative rates is another remarkable fact since the beginning of the global financial crisis. We emphasise that our multiple-curve interest rate model also takes this issue into account and allows to consider negative rates.

The multiple-curve approach invalidates the classical pricing theory of interest rate derivatives. Originally, it is based on the cornerstone of a unique consistent term structure 


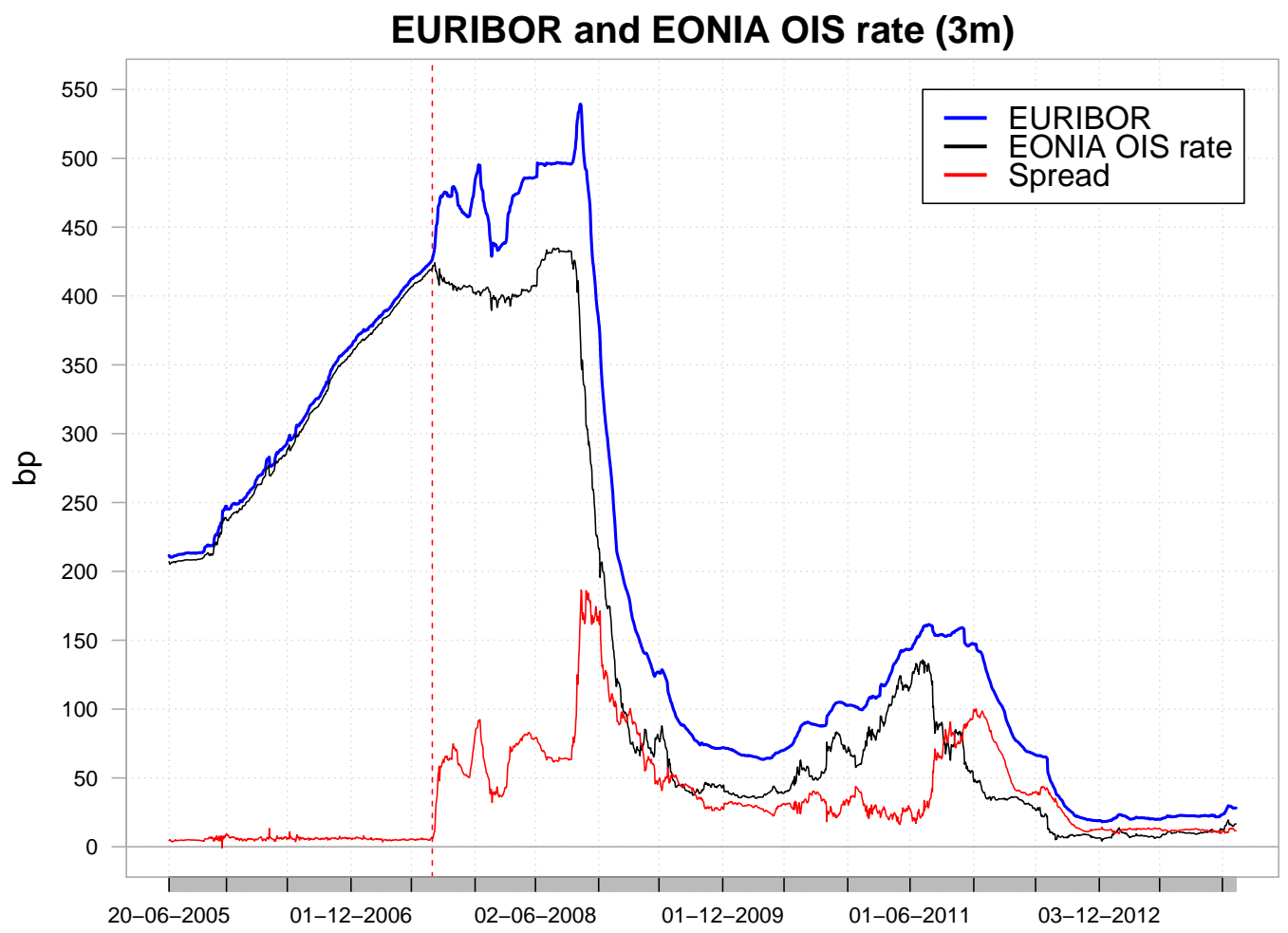

Figure 2.8.: Evolution of the EURIBOR and EONIA OIS rates corresponding to the three-month tenor.

curve that is used both for the producing of future cash flows and for the calculation of their present values. More specifically, when pricing interest rate derivatives with a given model the usual first step is to bootstrap the initial term structure of discount factors and forward rates. Before the crisis, this was a straightforward task because of the existence of a unique curve. When dealing with the multiple-curve approach this issue needs a more sophisticated and extensive treatment. To construct a term structure related to a given tenor, one may only use market quotes corresponding to this tenor. For instance, the six-month curve is constructed by bootstrapping rates from the market quotes related to the six-month tenor of the deposits for the short term maturities, the futures and FRAs for the short-mid term maturities and the liquid interest rate swaps for the mid-long term maturities. While the multiple term structures construction is based on the tenor homogeneity principle that in the meantime turns out to be market consensus, there is no general market convention for the building of the discount curve. Basically, there are two different practices:

1. OIS-discounting.

2. Classical pre-crisis approach based on the most liquid instruments. 


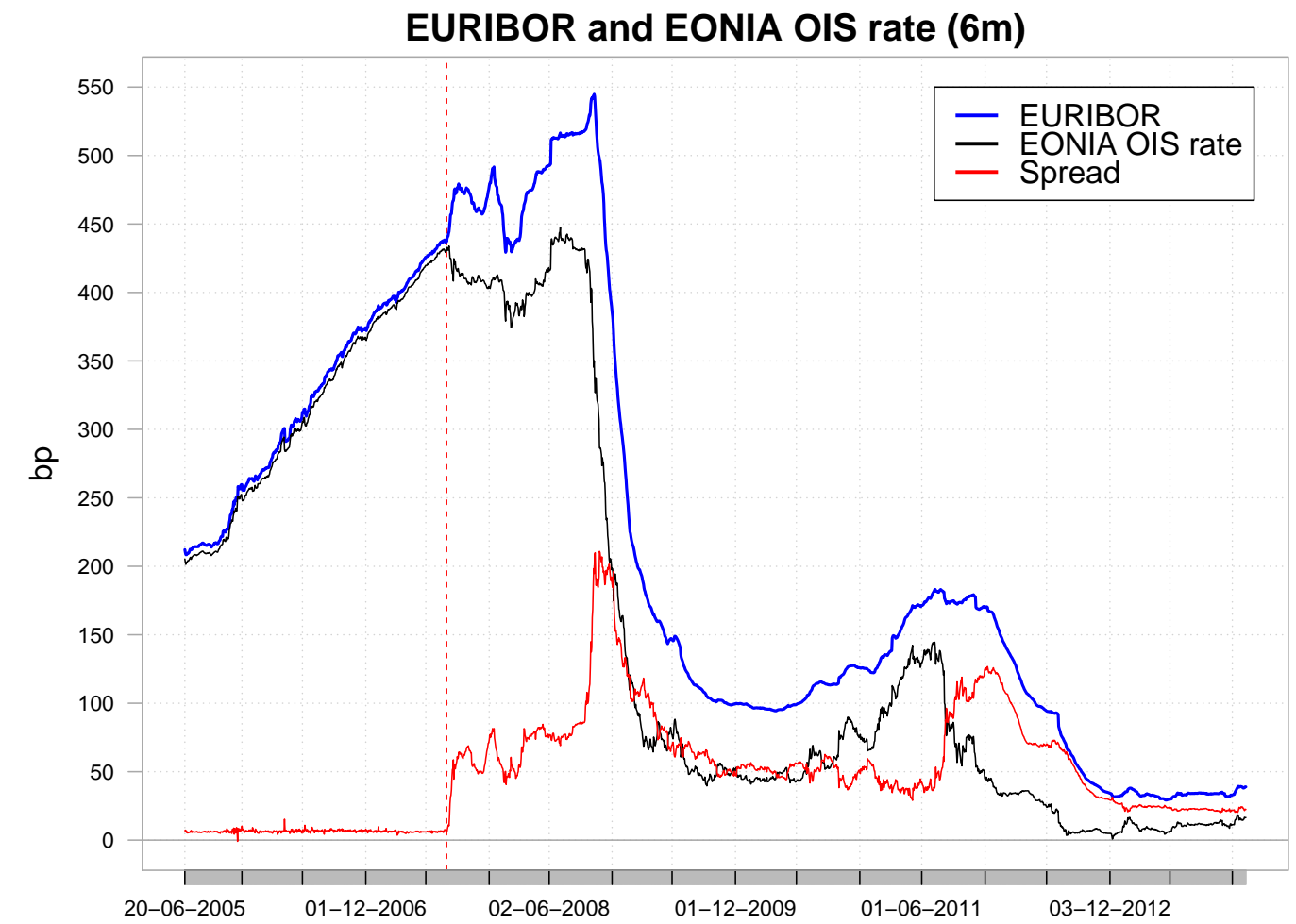

Figure 2.9.: Evolution of the EURIBOR and EONIA OIS rates corresponding to the six-month tenor.

We briefly explain the first approach. An effect of the global financial crisis has been the wide dissemination of collateral agreements to reduce the counterparty risk of OTCtraded derivatives. The collateral mechanism can be seen as a funding and hedging mechanism. It is mainly based on daily margination and an overnight collateral rate. Consequently, prices of derivatives quoted in the interbank market can be considered as free of credit and liquidity risk OTC-transactions. Another consequence is that the margination funding rate and the discount rate of future cash flows need to coincide by no-arbitrage arguments. Therefore, a collateral agreement implies overnight based discounting and the construction of the discount curve needs to reflect the funding level in an overnight collateralised interbank market. Overnight indexed swaps are thus canonical instruments for this construction. It can be seen as best available approximation of a riskfree yield curve. This is exactly the reason why this method is called OIS-discounting. We use this method in the multiple-curve model.

Furthermore, not only the initial multiple-curve construction but also the modelling of the evolution of the relevant rates becomes a delicate task. To solve this problem, one applies a logically similar method to the one used for the initial multiple-curve construction: when building a discount curve and term structures for different tenors at 


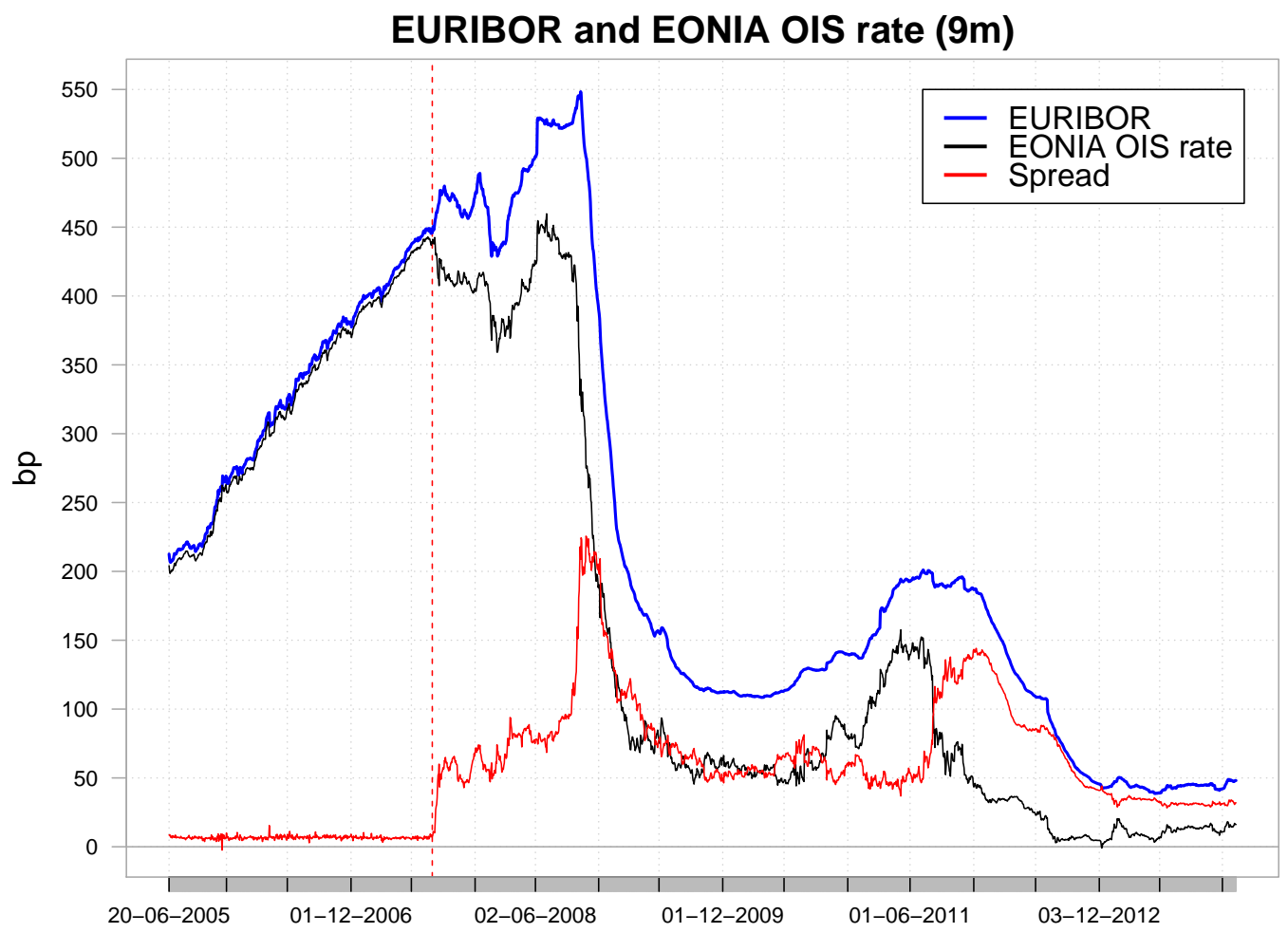

Figure 2.10.: Evolution of the EURIBOR and EONIA OIS rates corresponding to the nine-month tenor.

inception, a canonical way is to develop an interest rate model where these curves are modelled jointly but distinctly. It is worth mentioning that the modern multiple-curve approach generalises the approach based on one curve. This classical method is referred to as single-curve approach.

To end this subsection, we list some of the publications that are crucial for this part of the thesis: For the construction of the (multiple) term structures of interest rates, we refer to Musiela and Rutkowski [75], Hagan and West [51], Hull [55] and Ametrano and Bianchetti [2, 3]. The delicate issue of discount curve construction is considered by Henrard [53, 54]. Kijima, Tanaka, and Wong [62] develop an interest rate model in a multiple-curve framework. A double-curve model with regard to a foreign exchange analogy is introduced by Bianchetti [10. Modern market models are stated by Mercurio [69, 70, 71. Models based on the Heath-Jarrow-Morton framework are considered by Moreni and Pallavicini [73] and Crépey, Grbac, and Nguyen [23]. We further refer to Pallavicini and Tarenghi [77], Morini [74], Bianchetti and Carlicchi [11] and Filipović and Trolle [45]. A profound overview about the causes and main events of the global financial crisis is given by Brunnermeier [18] and Sinn [84]. 


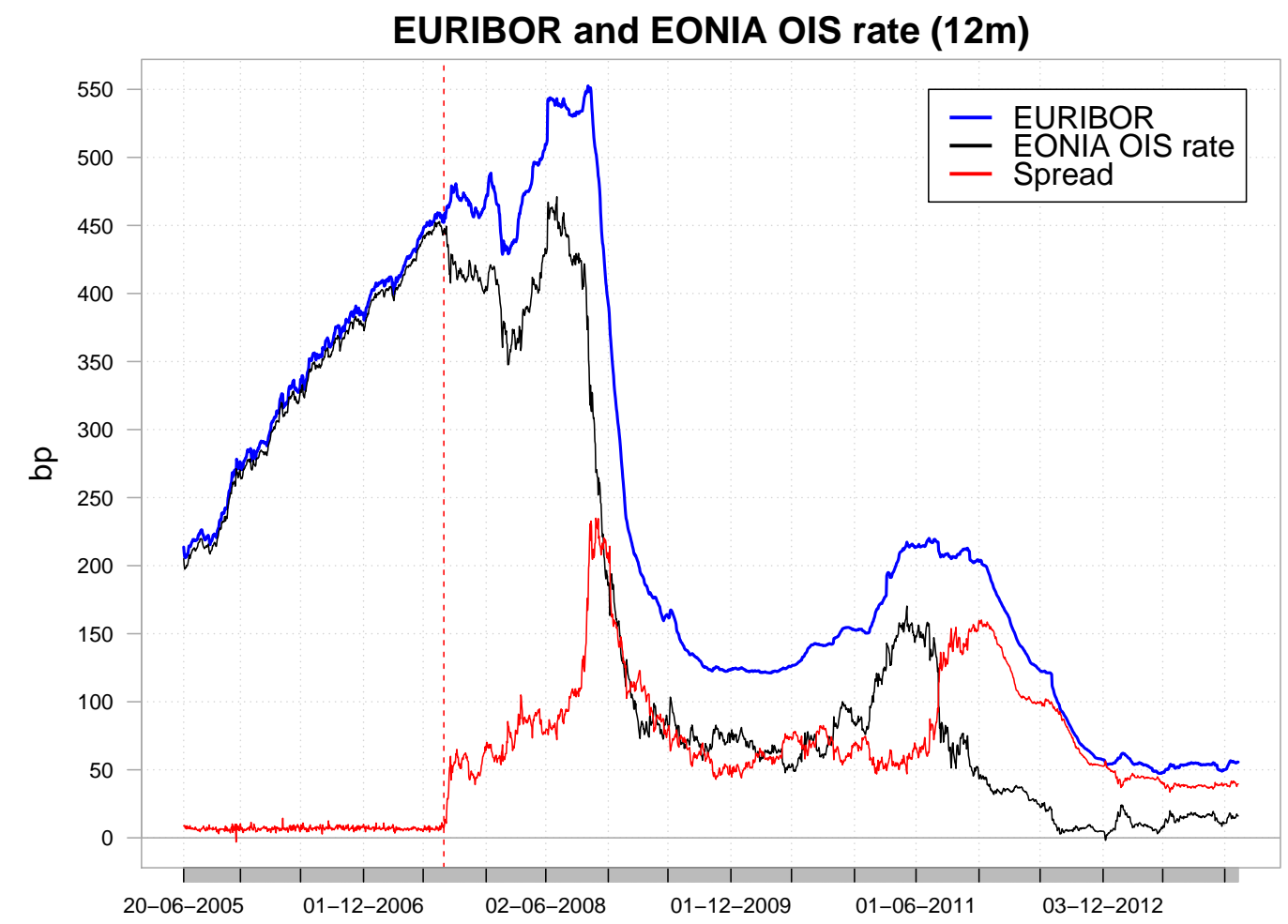

Figure 2.11.: Evolution of the EURIBOR and EONIA OIS rates corresponding to the twelve-month tenor.

\subsubsection{Valuation Formulas of Interest Rate Derivatives}

In this subsection, we illustrate the modern multiple-curve valuation method. To this end, model independent pricing formulas for the interest rate derivatives of subsection 2.2 .2 are stated.

Let $m \in \mathbb{N}$ be given. We consider $m+1$ curve(s) associated to $\max \{m, 1\}$ tenor(s) in a single currency economy. The curve 0 (also denoted by $d$ ) is referred to as the discount curve. Note that the case $m=0$ is therefore the classical single-curve setting. This implies that the multiple-curve approach can be seen as a generalisation of the single-curve one.

Let us fix a finite time horizon $T^{*} \in \mathbb{R}_{+}$. The initial term structure of discount bond prices $B_{0}^{\text {d }}$ (initial discount curve) is defined by

$$
B_{0}^{\mathrm{d}}:\left\{\begin{array}{l}
{\left[0, T^{*}\right] \rightarrow(0, \infty)} \\
T \mapsto B_{0}^{\mathrm{d}}(T) .
\end{array}\right.
$$

For any $m \geq 1$ and each $i \in\{1, \ldots, m\}$, we interpret $B_{t}^{i}(T)$ as time- $t$ price of a fictitious 
EURIBOR-EONIA OIS rate Spread

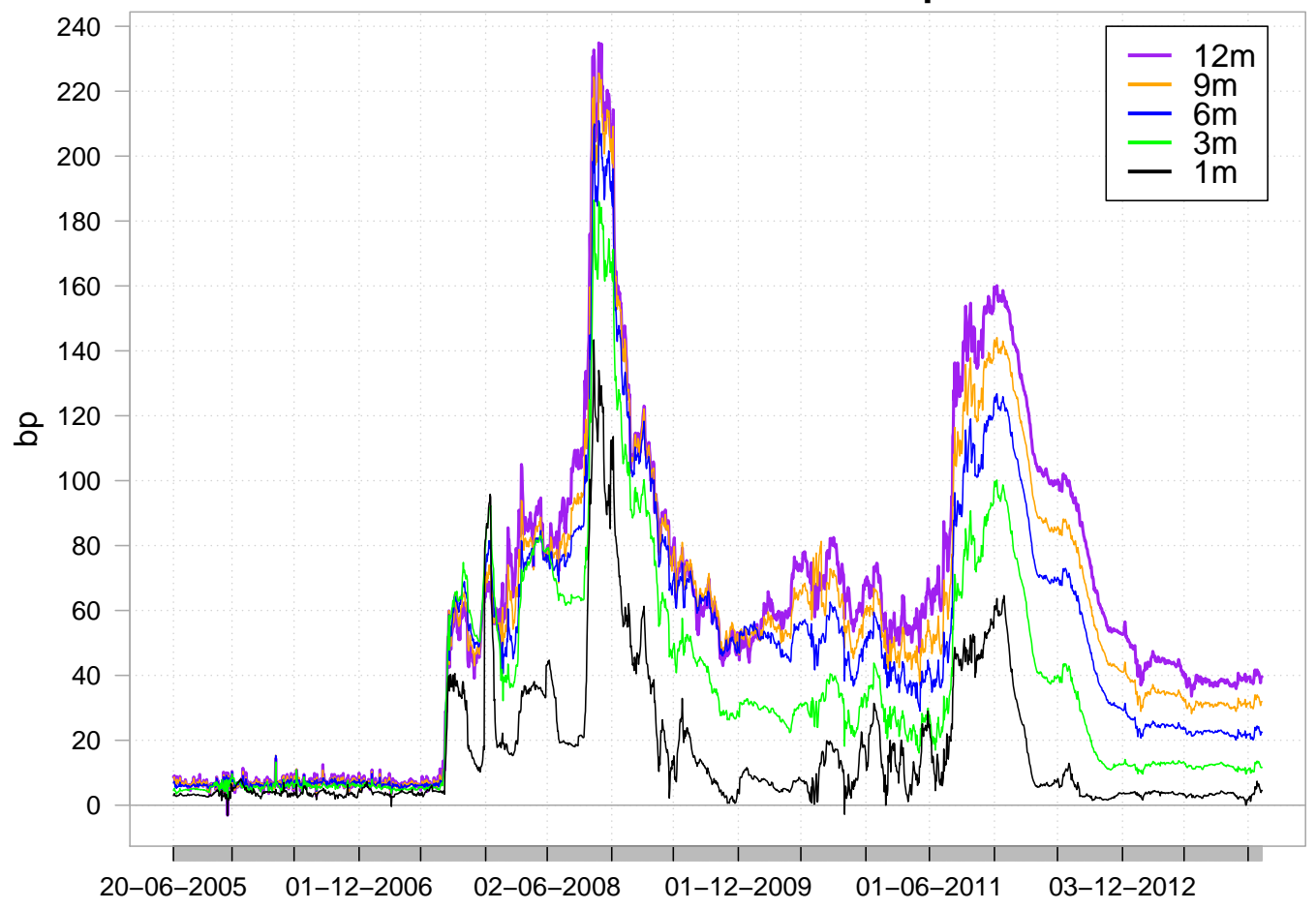

Figure 2.12.: Evolution of the spreads between EURIBOR and EONIA OIS rates for different tenors.

risky zero-coupon bond with maturity $T$. Notice that such risky bonds are not traded assets but can be considered as bonds being issued by an average Libor or Euribor panel member. The initial curve $B_{0}^{i}$ (initial curve $i$ ) is defined by

$$
B_{0}^{i}:\left\{\begin{array}{l}
{\left[0, T^{*}\right] \rightarrow(0, \infty)} \\
T \mapsto B_{0}^{i}(T)
\end{array}\right.
$$

This curve is constructed by bootstrapping techniques. Ametrano and Bianchetti [3] present a sophisticated bootstrapping method which deals with the multiple-curve setting. The initial discount curve $B_{0}^{\mathrm{d}}$ and every initial curve $B_{0}^{i}$ serve as ingredients of the multiple-curve interest rate model of chapter 3 .

We determine a stochastic process $r^{\mathrm{d}}=\left(r_{t}^{\mathrm{d}}\right)_{t \in\left[0, T^{*}\right]}$ on a complete stochastic basis $\mathscr{B}:=$ $\left(\Omega, \mathscr{G}, \mathbb{G}=\left(\mathscr{G}_{t}\right)_{t \in\left[0, T^{*}\right]}, P^{\mathrm{d}}\right)$ satisfying suitable measurability properties. This process plays the role of the risk-free short rate dynamics. Let us define the continuous discount 


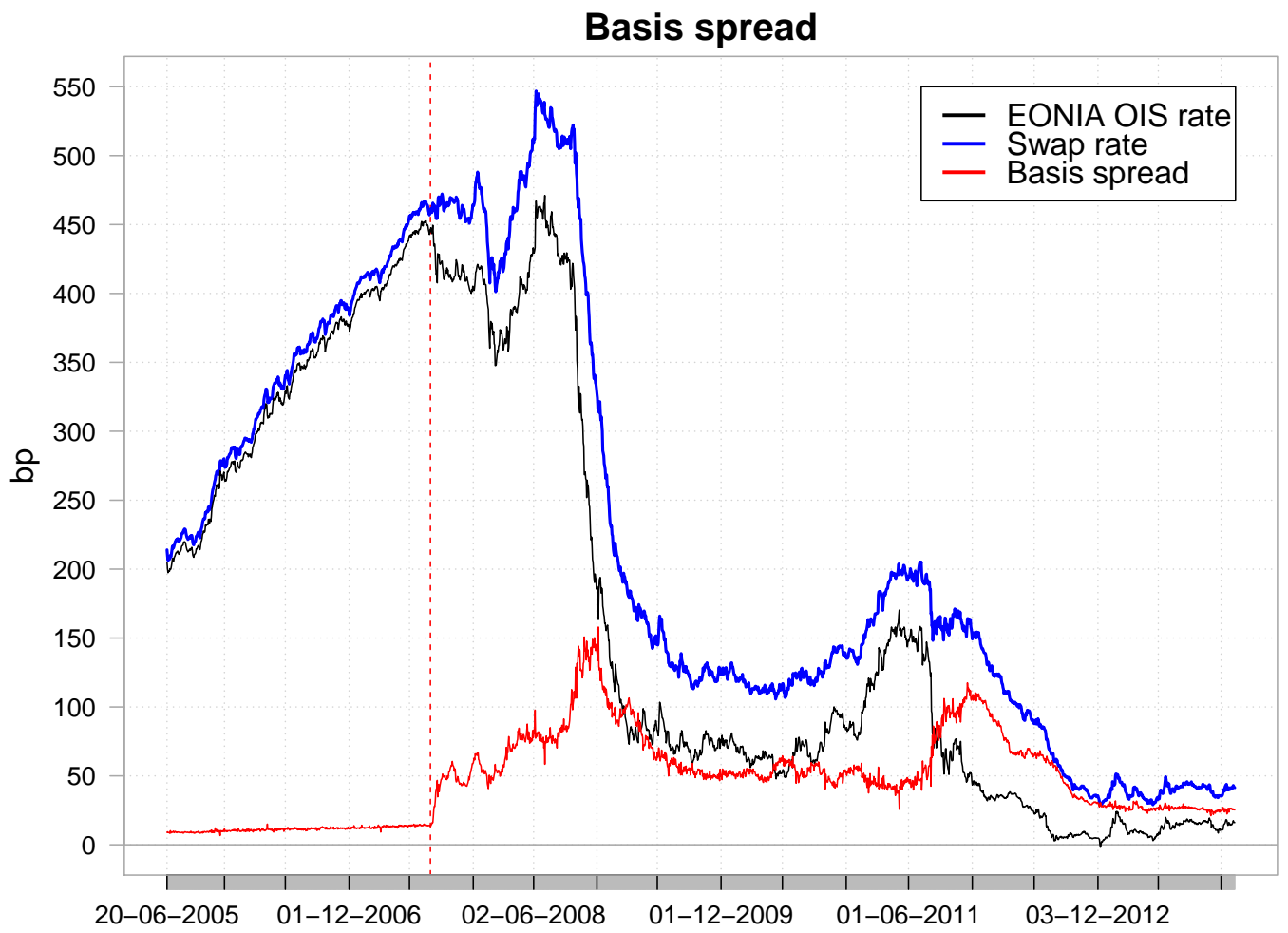

Figure 2.13.: Evolution of swap rates for swaps with a maturity of one year based on three-month and daily payment frequency.

factor process $\beta^{\mathrm{d}}=\left(\beta_{t}^{\mathrm{d}}\right)_{t \in\left[0, T^{*}\right]}$ by

$$
\beta_{t}^{\mathrm{d}}:=\exp \left(-\int_{0}^{t} r_{s}^{\mathrm{d}} d s\right)
$$

The discount bond price at date $t$ with maturity $T$ is denoted by $B_{t}^{\mathrm{d}}(T)$. The probability measure $P^{\mathrm{d}}$ is specified in such a way that the discounted bond price process $Z^{\mathrm{d}}(T)=$ $\left(Z_{t}^{\mathrm{d}}(T)\right)_{t \in\left[0, T^{*}\right]}$ given by

$$
Z_{t}^{\mathrm{d}}(T):=\beta_{t}^{\mathrm{d}} B_{t}^{\mathrm{d}}(T)
$$

is a $\mathbb{G}$-(local) martingale for each maturity $T \in\left[0, T^{*}\right]$.

The forward martingale measure for the date $T \in\left[0, T^{*}\right]$, denoted by $P_{T}^{\mathrm{d}}$, is a probability measure defined on $\left(\Omega, \mathscr{G}_{T}\right)$ that is equivalent to $P^{\mathrm{d}}$ (see also Geman, El Karoui, and Rochet [49]). It is characterised by the Radon-Nikodym derivative of the restrictions 


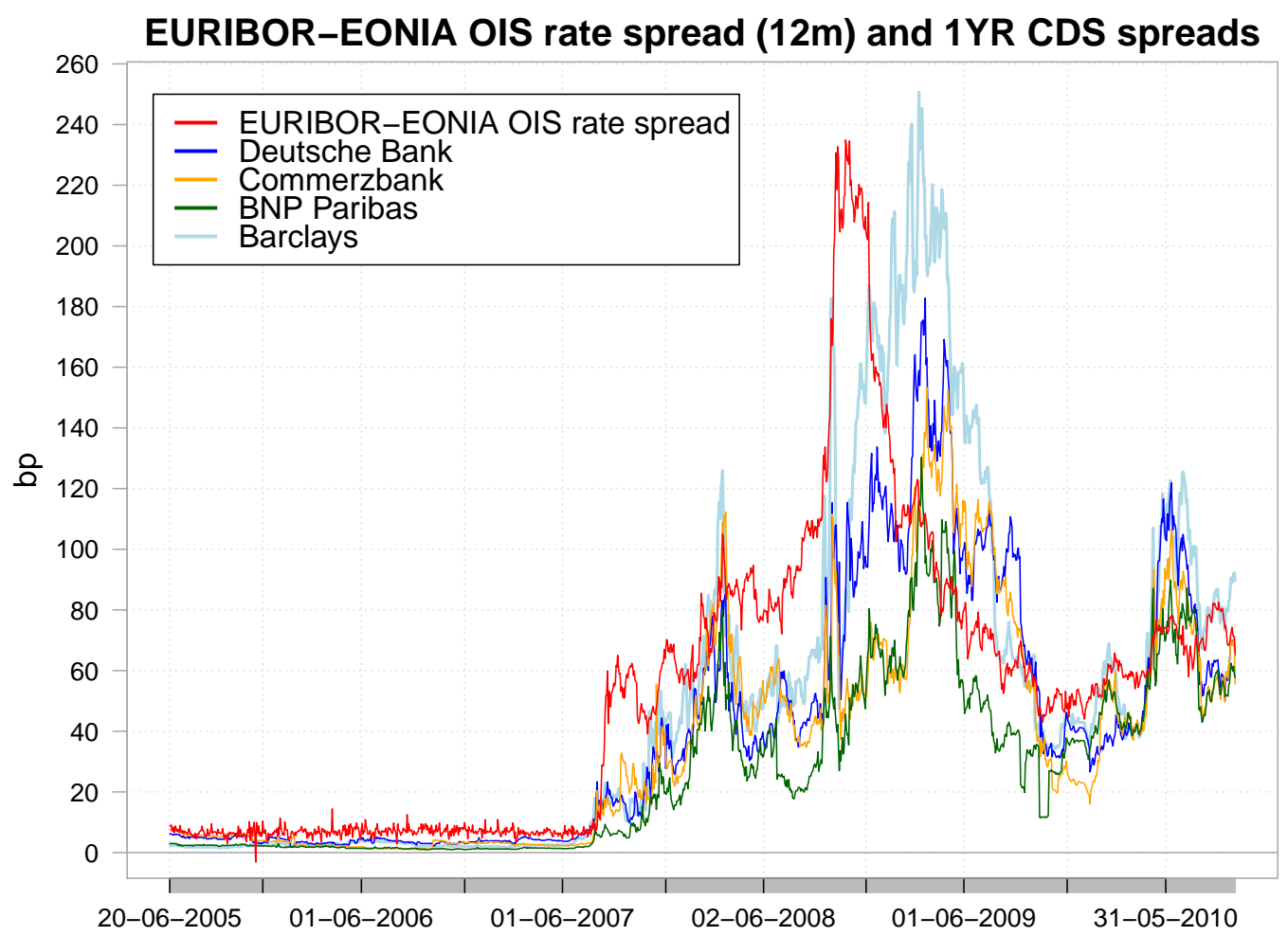

Figure 2.14.: Illustration of the influence of credit risk on the market anomalies.

of $P_{T}^{\mathrm{d}}$ and $P^{\mathrm{d}}$ to $\left(\Omega, \mathscr{G}_{T}\right)$ that is given by

$$
\frac{\left.d P_{T}^{\mathrm{d}}\right|_{\mathscr{G}_{T}}}{\left.d P^{\mathrm{d}}\right|_{\mathscr{G}_{T}}}=\frac{\beta_{T}^{\mathrm{d}}}{B_{0}^{\mathrm{d}}(T)} .
$$

For any $t \leq T$, the restriction to the $\sigma$-field $\mathscr{G}_{t}$ is of the form

$$
\frac{\left.d P_{T}^{\mathrm{d}}\right|_{\mathscr{G}_{t}}}{\left.d P^{\mathrm{d}}\right|_{\mathscr{G}_{t}}}=\mathbb{E}_{P^{\mathrm{d}}}\left[\frac{\beta_{T}^{\mathrm{d}}}{B_{0}^{\mathrm{d}}(T)} \mid \mathscr{G}_{t}\right]=\frac{\beta_{t}^{\mathrm{d}} B_{t}^{\mathrm{d}}(T)}{B_{0}^{\mathrm{d}}(T)} .
$$

Note that it can be useful to define the forward martingale measure on $\left(\Omega, \mathscr{G}_{T^{*}}\right)$. We use $P^{\mathrm{d}}$ and $P_{T}^{\mathrm{d}}$ as pricing measures here. To simplify the notation we set the notional amount equal to one.

\section{Forward Rate Agreements}

Let us fix two dates $T_{1} \in \mathbb{R}_{+}$and $T_{2}=T_{1}+\delta^{i}$, where the tenor $\delta^{i}$ corresponds to curve $i \in\{0,1, \ldots, m\}$. The time $T_{1}$ means the inception date and $T_{2}$ is the maturity of the (modified) forward rate agreement (see Mercurio [69]). The fixed rate of the FRA is 


\section{Two-Price Theory in Multiple-Curve Term Structure Models}

denoted by $K$ and the payoff of such an agreement at maturity $T_{2}$ is given by

$$
\operatorname{FRA}_{T_{2}}\left(T_{1}, T_{2}, \delta^{i}, K\right):=\delta^{i}\left(L_{T_{1}}^{i}\left(T_{1}, T_{2}\right)-K\right)
$$

where $L_{T_{1}}^{i}\left(T_{1}, T_{2}\right)$ denotes the time- $T_{1}$ (spot) reference rate relative to curve $i$. By the risk-neutral valuation principle, the value of the FRA at time $t \leq T_{1}$ can therefore be calculated as the conditional expectation with respect to the pricing measure $P^{\mathrm{d}}$. It is given by

$$
\mathrm{FRA}_{t}\left(T_{1}, T_{2}, \delta^{i}, K\right):=\delta^{i}\left(\beta_{t}^{\mathrm{d}}\right)^{-1} \mathbb{E}_{P^{\mathrm{d}}}\left[\beta_{T_{2}}^{\mathrm{d}}\left(L_{T_{1}}^{i}\left(T_{1}, T_{2}\right)-K\right) \mid \mathscr{G}_{t}\right] .
$$

By using the change-of-measure technique, we switch to the forward measure $P_{T_{2}}^{\mathrm{d}}$ and obtain

$$
\operatorname{FRA}_{t}\left(T_{1}, T_{2}, \delta^{i}, K\right)=\delta^{i} B_{t}^{\mathrm{d}}\left(T_{2}\right) \mathbb{E}_{P_{T_{2}}^{\mathrm{d}}}\left[\left(L_{T_{1}}^{i}\left(T_{1}, T_{2}\right)-K\right) \mid \mathscr{G}_{t}\right] .
$$

The rate for which the value of this contract at time $t$ is equal to zero is denoted by $K_{t}^{i}$. It can obviously be expressed as

$$
K_{t}^{i}=\mathbb{E}_{P_{T_{2}}^{\mathrm{d}}}\left[L_{T_{1}}^{i}\left(T_{1}, T_{2}\right) \mid \mathscr{G}_{t}\right]
$$

We stress that in the multiple curve framework $(m \geq 1)$, the dynamics of the forward reference rate $L^{i}\left(T_{1}, T_{2}\right)$ are in general not a $\left(\mathbb{G}, P_{T_{2}}^{\mathrm{d}}\right)$-(local) martingale. This fact is in accordance with the market quotes of the crisis (see figures 2.5 and 2.6. In the single-curve setting $(m=0)$, we have the well-known form

$$
K_{t}^{\mathrm{d}}=\mathbb{E}_{P_{T_{2}}^{\mathrm{d}}}\left[L_{T_{1}}^{\mathrm{d}}\left(T_{1}, T_{2}\right) \mid \mathscr{G}_{t}\right]=L_{t}^{\mathrm{d}}\left(T_{1}, T_{2}\right)
$$

\section{Interest Rate Swaps}

Let us consider an equidistant discrete tenor structure $\mathscr{T}^{i}=\left\{T_{0}, \ldots, T_{n}\right\}$ that corresponds to the curve $i \in\{0,1, \ldots, m\}$ with $T_{n}=T^{*}$. The payoff of an interest rate swap for the receiver of the floating rate at payment date $T_{k}$ with $k \in\{1, \ldots, n\}$ is given by

$$
\delta^{i}\left(L_{T_{k-1}}^{i}\left(T_{k-1}, T_{k}\right)-\mathrm{S}\right)
$$




\subsection{Multiple-Curve Interest Rate Term Structure Modelling}

where we set $\delta^{i}:=\delta^{i}\left(T_{k-1}, T_{k}\right)$ for a year fraction $\delta^{i}$ and $\mathbf{S}$ denotes the fixed rate. The value of this swap at time $t$ can be calculated as

$$
\begin{aligned}
\operatorname{Swap}_{t}\left(\mathscr{T}^{i}, \delta^{i}\right) & :=\sum_{k=1}^{n} \delta^{i}\left(\beta_{t}^{\mathrm{d}}\right)^{-1} \mathbb{E}_{P^{\mathrm{d}}}\left[\beta_{T_{k}}^{\mathrm{d}}\left(L_{T_{k-1}}^{i}\left(T_{k-1}, T_{k}\right)-\mathrm{S}\right) \mid \mathscr{G}_{t}\right] \\
& =\sum_{k=1}^{n} \delta^{i} B_{t}^{\mathrm{d}}\left(T_{k}\right) \mathbb{E}_{P_{T_{k}}^{\mathrm{d}}}\left[\left(L_{T_{k-1}}^{i}\left(T_{k-1}, T_{k}\right)-\mathrm{S}\right) \mid \mathscr{G}_{t}\right] \\
& =\sum_{k=1}^{n} \operatorname{FRA}_{t}\left(T_{k-1}, T_{k}, \delta^{i}, \mathrm{~S}\right) .
\end{aligned}
$$

The swap rate at time $t$ with $t \leq T_{0}$, denoted by $\mathrm{S}_{t}^{i}\left(\mathscr{T}^{i}\right)$, is defined as the rate that makes the time- $t$ value of the swap equal to zero. Thus, we obtain

$$
\mathrm{S}_{t}^{i}\left(\mathscr{T}^{i}\right)=\frac{\sum_{k=1}^{n} \delta^{i} B_{t}^{\mathrm{d}}\left(T_{k}\right) \mathbb{E}_{P_{T_{k}}^{\mathrm{d}}}\left[L_{T_{k-1}}^{i}\left(T_{k-1}, T_{k}\right) \mid \mathscr{G}_{t}\right]}{\sum_{k=1}^{n} \delta^{i} B_{t}^{\mathrm{d}}\left(T_{k}\right)} .
$$

If we consider $m=0$, we get the common formula

$$
\mathrm{S}_{t}\left(\mathscr{T}^{\mathrm{d}}\right)=\frac{B_{t}^{\mathrm{d}}\left(T_{0}\right)-B_{t}^{\mathrm{d}}\left(T_{n}\right)}{\sum_{k=1}^{n} \delta B_{t}^{\mathrm{d}}\left(T_{k}\right)}
$$

\section{Overnight Indexed Swaps}

The overnight indexed swap is a special case of an interest rate swap. Let $\mathscr{T}^{i}=$ $\left\{T_{0}, \ldots, T_{n}\right\}$ be an equidistant discrete tenor structure as above. The overnight floating rate for the time interval $\left[T_{k-1}, T_{k}\right]$ for every $k \in\{1, \ldots, n\}$ is assumed to be given by simply compounding the consecutive overnight rates between the dates $T_{k-1}$ and $T_{k}$. We write $R^{\text {on }}(S, T)$ for the overnight rate between $T$ and $S$, where $0 \leq S \leq T \leq T_{n}$. More specifically, let $T_{k-1}=t_{0}<t_{1}<\cdots<t_{N^{i}}=T_{k}$ be a partition of the interval $\left[T_{k-1}, T_{k}\right]$ into $N^{i}$ trading days (i.e. business days). As mentioned above, we set

$$
R^{\mathrm{on}}\left(T_{k-1}, T_{k}\right)=\frac{1}{\delta^{i}}\left(\prod_{j=1}^{N^{i}}\left(1+\delta\left(t_{j-1}, t_{j}\right) R^{\mathrm{on}}\left(t_{j-1}, t_{j}\right)\right)-1\right),
$$

where $\delta$ is an appropriate year fraction. Since the overnight rate $R^{\text {on }}\left(t_{j-1}, t_{j}\right)$ can assumed to be risk free (cf. subsection 2.2.3, we set $R^{\text {on }}\left(t_{j-1}, t_{j}\right)=r_{t_{j-1}}^{\mathrm{d}}$. We approximate the rate $R^{\text {on }}\left(T_{k-1}, T_{k}\right)$ by

$$
R^{\mathrm{on}}\left(T_{k-1}, T_{k}\right) \approx \frac{1}{\delta^{i}}\left(\exp \left(\int_{T_{k-1}}^{T_{k}} r_{s}^{\mathrm{d}} d s\right)-1\right),
$$




\section{Two-Price Theory in Multiple-Curve Term Structure Models}

where we used the fact that

$$
1+\delta\left(t_{j-1}, t_{j}\right) r_{t_{j-1}}^{\mathrm{d}} \sim \exp \left(\delta\left(t_{j-1}, t_{j}\right) r_{t_{j-1}}^{\mathrm{d}}\right)
$$

Then, the time- $t$ value of the (forward) overnight indexed swap with $t \leq T_{0}$ is given by

$$
\begin{aligned}
\operatorname{OIS}_{t}\left(\mathscr{T}^{i}\right)= & \sum_{k=1}^{n}\left(\beta_{t}^{\mathrm{d}}\right)^{-1} \mathbb{E}_{P^{\mathrm{d}}}\left[\beta_{T_{k}}^{\mathrm{d}} \delta^{i}\left(R^{\mathrm{on}}\left(T_{k-1}, T_{k}\right)-\mathrm{S}\right) \mid \mathscr{G}_{t}\right] \\
= & \sum_{k=1}^{n} \mathbb{E}_{P^{\mathrm{d}}}\left[\exp \left(-\int_{t}^{T_{k-1}} r_{s}^{\mathrm{d}} d s\right)-\exp \left(-\int_{t}^{T_{k}} r_{s}^{\mathrm{d}} d s\right)\right. \\
& \left.-\exp \left(-\int_{t}^{T_{k}} r_{s}^{\mathrm{d}} d s\right) \delta^{i} \mathrm{~S} \mid \mathscr{G}_{t}\right] \\
= & \sum_{k=1}^{n}\left(B_{t}^{\mathrm{d}}\left(T_{k-1}\right)-B_{t}^{\mathrm{d}}\left(T_{k}\right)\right)-\sum_{k=1}^{n} \delta^{i} \mathrm{~S} B_{t}^{\mathrm{d}}\left(T_{k}\right) \\
= & B_{t}^{\mathrm{d}}\left(T_{0}\right)-B_{t}^{\mathrm{d}}\left(T_{n}\right)-\delta^{i} \mathrm{~S} \sum_{k=1}^{n} B_{t}^{\mathrm{d}}\left(T_{k}\right)
\end{aligned}
$$

where we exploit the relation

$$
B_{t}^{\mathrm{d}}(T)=\mathbb{E}_{P^{\mathrm{d}}}\left[\exp \left(-\int_{t}^{T} r_{s}^{\mathrm{d}} d s\right) \mid \mathscr{G}_{t}\right] .
$$

The overnight indexed swap rate at time $t$, denoted by $\mathrm{S}_{t}^{\text {on }}\left(\mathscr{T}^{i}\right)$, determines the value of the overnight indexed swap at time $t$ equal to zero. It is given by

$$
\mathrm{S}_{t}^{\text {on }}\left(\mathscr{T}^{i}\right)=\frac{B_{t}^{\mathrm{d}}\left(T_{0}\right)-B_{t}^{\mathrm{d}}\left(T_{n}\right)}{\sum_{k=1}^{n} \delta^{i} B_{t}^{\mathrm{d}}\left(T_{k}\right)}
$$

The (forward) Libor/Euribor-OIS spread at time $t$ for the interval $\left[T_{k-1}, T_{k}\right]$ is defined by

$$
\begin{aligned}
s_{t}^{i, \text { on }}\left(T_{k-1}, T_{k}\right) & :=L_{t}^{i}\left(T_{k-1}, T_{k}\right)-\mathrm{S}_{t}^{\text {on }}\left(\left\{T_{k-1}, T_{k}\right\}\right) \\
& =\frac{1}{\delta^{i}}\left(\frac{B_{t}^{i}\left(T_{k-1}\right)}{B_{t}^{i}\left(T_{k}\right)}-1\right)-\frac{B_{t}^{\mathrm{d}}\left(T_{k-1}\right)-B_{t}^{\mathrm{d}}\left(T_{k}\right)}{\delta^{i} B_{t}^{\mathrm{d}}\left(T_{k}\right)} \\
& =\frac{1}{\delta^{i}}\left(\frac{B_{t}^{i}\left(T_{k-1}\right)}{B_{t}^{i}\left(T_{k}\right)}-\frac{B_{t}^{\mathrm{d}}\left(T_{k-1}\right)}{B_{t}^{\mathrm{d}}\left(T_{k}\right)}\right) \\
& =\frac{1}{\delta^{i}}\left(F_{t}^{i}\left(T_{k-1}, T_{k}\right)-F_{t}^{\mathrm{d}}\left(T_{k-1}, T_{k}\right)\right) .
\end{aligned}
$$

The Libor/Euribor-OIS swap spread at time $t$ is defined as the difference between the time- $t$ swap rate corresponding to curve $i$ and the overnight indexed swap rate. Formally, 
it is of the form

$$
\mathrm{S}_{t}^{i}\left(\mathscr{T}^{i}\right)-\mathrm{S}_{t}^{\text {on }}\left(\mathscr{T}^{i}\right)=\frac{\sum_{k=1}^{n} \delta^{i} B_{t}^{\mathrm{d}}\left(T_{k}\right) \mathbb{E}_{P_{T_{k}}^{\mathrm{d}}}\left[L_{T_{k-1}}^{i}\left(T_{k-1}, T_{k}\right) \mid \mathscr{G}_{t}\right]-B_{t}^{\mathrm{d}}\left(T_{0}\right)+B_{t}^{\mathrm{d}}\left(T_{n}\right)}{\sum_{k=1}^{n} \delta^{i} B_{t}^{\mathrm{d}}\left(T_{k}\right)} .
$$

\section{Basis Swaps}

Let $i, j \in\{0,1, \ldots, m\}$ be given with $i \leq j$. We consider two equidistant discrete tenor structures $\mathscr{T}^{i}=\left\{T_{0}^{i}, \ldots, T_{n_{i}}^{i}\right\}$ and $\mathscr{T}^{j}=\left\{T_{0}^{j}, \ldots, T_{n_{j}}^{j}\right\}$ with $n_{i}, n_{j} \in \mathbb{N}$ and tenors $\delta^{i}$ and $\delta^{j}$. It is assumed that it holds $0 \leq T_{0}^{i}=T_{0}^{j}, T_{n_{i}}^{i}=T^{*}$ and $\mathscr{T}^{j} \subset \mathscr{T}^{i}$ for every $i, j \in\{0,1, \ldots, m\}$. The last assumption corresponds to the fact that $\mathscr{T}^{j}$ relates to the riskier curve compared to $\mathscr{T}^{i}\left(\delta^{i}<\delta^{j}\right)$.

A basis swap agreement is usually understood in two ways:

1. As the difference of the two different floating legs with given maturity.

2. As a portfolio of two interest rate swaps with same fixed legs and maturity but with different floating legs.

In the first case, the time- $t$ value of a basis swap with $t \leq T_{0}^{i}=T_{0}^{j}$ can be determined by

$$
\begin{aligned}
\mathrm{BS}_{t}^{1}\left(\mathscr{T}^{i}, \mathscr{T}^{j}\right):= & \sum_{k=1}^{n_{j}} \delta^{j} B_{t}^{\mathrm{d}}\left(T_{k}^{j}\right) \mathbb{E}_{P_{T_{k}^{j}}^{\mathrm{d}}}\left[L_{T_{k-1}^{j}}^{j}\left(T_{k-1}^{j}, T_{k}^{j}\right) \mid \mathscr{G}_{t}\right] \\
& -\sum_{k=1}^{n_{i}} \delta^{i} B_{t}^{\mathrm{d}}\left(T_{k}^{i}\right) \mathbb{E}_{P_{T_{k}^{i}}^{\mathrm{d}}}\left[L_{T_{k-1}^{i}}^{i}\left(T_{k-1}^{i}, T_{k}^{i}\right) \mid \mathscr{G}_{t}\right] .
\end{aligned}
$$

Secondly, we obtain

$$
\begin{aligned}
\mathrm{BS}_{t}^{2}\left(\mathscr{T}^{i}, \mathscr{T}^{j}\right):= & {\left[\sum_{k=1}^{n_{j}} \delta^{j} B_{t}^{\mathrm{d}}\left(T_{k}^{j}\right) \mathbb{E}_{P_{T_{k}^{j}}^{\mathrm{d}}}\left[L_{T_{k-1}^{j}}^{j}\left(T_{k-1}^{j}, T_{k}^{j}\right) \mid \mathscr{G}_{t}\right]-\sum_{k=1}^{n} \delta B_{t}^{\mathrm{d}}\left(T_{k}\right) \mathrm{S}_{t}^{j}\right] } \\
& -\left[\sum_{k=1}^{n_{i}} \delta^{i} B_{t}^{\mathrm{d}}\left(T_{k}^{i}\right) \mathbb{E}_{P_{T_{k}^{i}}^{\mathrm{d}}}\left[L_{T_{k-1}^{i}}^{i}\left(T_{k-1}^{i}, T_{k}^{i}\right) \mid \mathscr{G}_{t}\right]-\sum_{k=1}^{n} \delta B_{t}^{\mathrm{d}}\left(T_{k}\right) \mathrm{S}_{t}^{i}\right],
\end{aligned}
$$

where $\mathrm{S}_{t}^{i}$ and $\mathrm{S}_{t}^{j}$ denote the time- $t$ swap rates of the respective contracts and $\mathscr{T}=$ $\left\{T_{0}, T_{1}, \ldots, T_{n}\right\}$ is the tenor structure corresponding to the fixed legs. Motivated by figure 2.13, we set $\mathrm{S}_{t}^{j}=\mathrm{S}_{t}^{i}+\mathrm{bs}_{t}$, where $\mathrm{bs}_{t}$ means the basis spread at time $t$. Then, we 
get

$$
\begin{aligned}
\mathrm{BS}_{t}^{2}\left(\mathscr{T}^{i}, \mathscr{T}^{j}\right):= & \sum_{k=1}^{n_{j}} \delta^{j} B_{t}^{\mathrm{d}}\left(T_{k}^{j}\right) \mathbb{E}_{P_{T_{k}^{j}}^{\mathrm{d}}}\left[L_{T_{k-1}^{j}}^{j}\left(T_{k-1}^{j}, T_{k}^{j}\right) \mid \mathscr{G}_{t}\right]-\sum_{k=1}^{n} \delta B_{t}^{\mathrm{d}}\left(T_{k}\right) \cdot \mathrm{bs} t \\
& -\sum_{k=1}^{n_{i}} \delta^{i} B_{t}^{\mathrm{d}}\left(T_{k}^{i}\right) \mathbb{E}_{P_{T_{k}^{i}}^{\mathrm{d}}}\left[L_{T_{k-1}^{i}}^{i}\left(T_{k-1}^{i}, T_{k}^{i}\right) \mid \mathscr{G}_{t}\right] .
\end{aligned}
$$

This formula implies the equilibrium basis spread that sets the value of the basis swap equal to zero:

$$
\mathrm{bs}_{t}=\frac{\sum_{k=1}^{n_{j}} \delta^{j} B_{t}^{\mathrm{d}}\left(T_{k}^{j}\right) \mathbb{E}_{P_{T_{k}^{j}}^{\mathrm{d}}}\left[L_{T_{k-1}^{j}}^{j}\left(T_{k-1}^{j}, T_{k}^{j}\right) \mid \mathscr{G}_{t}\right]-\sum_{k=1}^{n_{i}} \delta^{i} B_{t}^{\mathrm{d}}\left(T_{k}^{i}\right) \mathbb{E}_{P_{T_{k}^{i}}^{\mathrm{d}}}\left[L_{T_{k-1}^{i}}^{i}\left(T_{k-1}^{i}, T_{k}^{i}\right) \mid \mathscr{G}_{t}\right]}{\sum_{k=1}^{n} \delta B_{t}^{\mathrm{d}}\left(T_{k}\right)} .
$$

\section{Caps and Floors}

Let $\mathscr{T}^{i}=\left\{T_{0}, \ldots, T_{n}\right\}$ be an equidistant discrete tenor structure related to curve $i \in$ $\{0,1, \ldots, m\}$. The payoff of a cap with strike $K$ at time $T_{k}$ for every $k \in\{1, \ldots, n\}$ is then given by

$$
\delta^{i}\left(L_{T_{k-1}}^{i}\left(T_{k-1}, T_{k}\right)-K\right)^{+},
$$

where we define $f^{+}:=\max \{f, 0\}$ for any measurable function $f$. Accordingly, the payoff of a floor with the same contractual features is

$$
\delta^{i}\left(K-L_{T_{k-1}}^{i}\left(T_{k-1}, T_{k}\right)\right)^{+} .
$$

The time- $t$ price of a cap for $t \leq T_{0}$ and with strike $K$ is determined by

$$
\begin{aligned}
\operatorname{Cap}_{t}\left(\mathscr{T}^{i}, \delta^{i}, K\right) & :=\sum_{k=1}^{n}\left(\beta_{t}^{\mathrm{d}}\right)^{-1} \mathbb{E}_{P^{\mathrm{d}}}\left[\beta_{T_{k}}^{\mathrm{d}} \delta^{i}\left(L_{T_{k-1}}^{i}\left(T_{k-1}, T_{k}\right)-K\right)^{+} \mid \mathscr{G}_{t}\right] \\
& =\sum_{k=1}^{n} \delta^{i} B_{t}^{\mathrm{d}}\left(T_{k}\right) \mathbb{E}_{P_{T_{k}}^{\mathrm{d}}}\left[\left(L_{T_{k-1}}^{i}\left(T_{k-1}, T_{k}\right)-K\right)^{+} \mid \mathscr{G}_{t}\right]
\end{aligned}
$$

and the related caplet price is given by

$$
\mathrm{Cpl}_{t}\left(T_{k-1}, T_{k}, K\right):=\delta^{i} B_{t}^{\mathrm{d}}\left(T_{k}\right) \mathbb{E}_{P_{T_{k}}^{\mathrm{d}}}\left[\left(L_{T_{k-1}}^{i}\left(T_{k-1}, T_{k}\right)-K\right)^{+} \mid \mathscr{G}_{t}\right] .
$$


Analogously, we obtain the price of the floor as

$$
\begin{aligned}
\text { Floor }_{t}\left(\mathscr{T}^{i}, \delta^{i}, K\right) & :=\sum_{k=1}^{n}\left(\beta_{t}^{\mathrm{d}}\right)^{-1} \mathbb{E}_{P^{\mathrm{d}}}\left[\beta_{T_{k}}^{\mathrm{d}} \delta^{i}\left(K-L_{T_{k-1}}^{i}\left(T_{k-1}, T_{k}\right)\right)^{+} \mid \mathscr{G}_{t}\right] \\
& =\sum_{k=1}^{n} \delta^{i} B_{t}^{\mathrm{d}}\left(T_{k}\right) \mathbb{E}_{P_{T_{k}}^{\mathrm{d}}}\left[\left(K-L_{T_{k-1}}^{i}\left(T_{k-1}, T_{k}\right)\right)^{+} \mid \mathscr{G}_{t}\right]
\end{aligned}
$$

and the floorlet price as

$$
\mathrm{Flt}_{t}\left(T_{k-1}, T_{k}, K\right):=\delta^{i} B_{t}^{\mathrm{d}}\left(T_{k}\right) \mathbb{E}_{P_{T_{k}}^{\mathrm{d}}}\left[\left(K-L_{T_{k-1}}^{i}\left(T_{k-1}, T_{k}\right)\right)^{+} \mid \mathscr{G}_{t}\right]
$$

Notice that for $m \geq 1$ the caplet (floorlet) can not be written as a put (call) option on a bond in the multiple-curve setting as it is classically done in the single-curve framework (see Musiela and Rutkowski [75, Section 12.2]).

\section{Digital Options}

Let us fix the dates $T_{1} \in \mathbb{R}_{+}$and $T_{2}=T_{1}+\delta^{i}$ with tenor $\delta^{i}$ related to curve $i \in$ $\{0,1, \ldots, m\}$. The time- $T_{1}$ value of a standard digital option is given by

$$
\mathrm{SD}_{T_{1}}^{i}\left(T_{1}, T_{2}, B, w\right):=\mathbb{1}_{\left\{w L_{T_{1}}^{i}\left(T_{1}, T_{2}\right)>w B\right\}}
$$

where we set

$$
w=\left\{\begin{array}{l}
1, \text { for a digital call } \\
-1, \text { for a digital put. }
\end{array}\right.
$$

Then, the time- $t$ price of this derivative for every $t \leq T_{1}$ can be represented as

$$
\begin{aligned}
\operatorname{SD}_{t}^{i}\left(T_{1}, T_{2}, B, w\right) & :=\left(\beta_{t}^{\mathrm{d}}\right)^{-1} \mathbb{E}_{P^{\mathrm{d}}}\left[\beta_{T_{1}}^{\mathrm{d}} \mathbb{1}_{\left\{w L_{T_{1}}^{i}\left(T_{1}, T_{2}\right)>w B\right\}} \mid \mathscr{G}_{t}\right] \\
& =B_{t}^{\mathrm{d}}\left(T_{1}\right) \mathbb{E}_{P_{T_{1}}^{\mathrm{d}}}\left[\mathbb{1}_{\left\{w L_{T_{1}}^{i}\left(T_{1}, T_{2}\right)>w B\right\}} \mid \mathscr{G}_{t}\right] .
\end{aligned}
$$

Let $T \in \mathbb{R}_{+}$be such that $T_{1} \leq T$. The time- $T$ value of a delayed digital option is given by

$$
\mathrm{DD}_{T}^{i}\left(T_{1}, T_{2}, T, B, w\right):=\mathbb{1}_{\left\{w L_{T_{1}}^{i}\left(T_{1}, T_{2}\right)>w B\right\}},
$$

where we have

$$
w=\left\{\begin{array}{l}
1, \text { for a delayed digital call } \\
-1, \text { for a delayed digital put }
\end{array}\right.
$$


We get its risk-neutral price at time $t \leq T_{1}$ as

$$
\begin{aligned}
\mathrm{DD}_{t}^{i}\left(T_{1}, T_{2}, T, B, w\right) & :=\left(\beta_{t}^{\mathrm{d}}\right)^{-1} \mathbb{E}_{P_{\mathrm{d}}^{\mathrm{d}}}\left[\beta_{T}^{\mathrm{d}} \mathbb{1}_{\left\{w L_{T_{1}}^{i}\left(T_{1}, T_{2}\right)>w B\right\}} \mid \mathscr{G}_{t}\right] \\
& =B_{t}^{\mathrm{d}}(T) \mathbb{E}_{P_{T}^{\mathrm{d}}}\left[\mathbb{1}_{\left\{w L_{T_{1}}^{i}\left(T_{1}, T_{2}\right)>w B\right\}} \mid \mathscr{G}_{t}\right]
\end{aligned}
$$

The value of a delayed range digital option at time $T$ with barriers $\underline{B}, \bar{B}$ satisfying $0<\underline{B}<\bar{B}$ is

$$
\begin{aligned}
\operatorname{DRD}_{T}^{i}\left(T_{1}, T_{2}, T, \underline{B}, \bar{B}\right) & :=\mathbb{1}_{\left\{\underline{B}<L_{T_{1}}^{i}\left(T_{1}, T_{2}\right)<\bar{B}\right\}} \\
& =\mathbb{1}_{\left\{\underline{B}<L_{T_{1}}^{i}\left(T_{1}, T_{2}\right)\right\}}+\mathbb{1}_{\left\{L_{T_{1}}^{i}\left(T_{1}, T_{2}\right)<\bar{B}\right\}}-1 .
\end{aligned}
$$

Therefore, we obtain that the time- $t$ price for any $t \leq T$ can be represented by

$$
\operatorname{DRD}_{t}^{i}\left(T, T_{1}, T_{2}, \underline{B}, \bar{B}\right):=\mathrm{DD}_{t}^{i}\left(T_{1}, T_{2}, T, \underline{B}, 1\right)+\mathrm{DD}_{t}^{i}\left(T_{1}, T_{2}, T, \bar{B},-1\right)-B_{t}^{\mathrm{d}}(T) .
$$

\subsection{Application of the Two-Price Theory}

We briefly describe the method to apply the two-price theory introduced in section 2.1 to the multiple-curve approach. To this end, let us postulate a mathematical model for the evolution of a fundamental quantity of the fixed income market, say $L^{i}\left(T_{k-1}^{i}, T_{k}^{i}\right)$ for every $i \in\{0,1, \ldots, m\}$ and $T_{k-1}^{i}, T_{k}^{i} \in \mathscr{T}^{i}=\left\{T_{0}^{i}, \ldots, T_{n_{i}}^{i}\right\}$. More specifically, all stochastic processes and random variables are defined on a complete stochastic basis $\left(\Omega, \mathscr{G}, \mathbb{G}=\left(\mathscr{G}_{t}\right)_{t \in\left[0, T^{*}\right]}, P^{\mathrm{d}}\right)$ (cf. section 2.2.

In general, the discounted payoff of an interest rate derivative is of complicated nature. The reason for this circumstance is the occurring stochastic discount factor for the present price $(t=0)$ with respect to the underlying pricing measure $P^{\mathrm{d}}$ (see for instance equation (2.20). To overcome this problem, we switch to the forward measure $P_{T}^{\mathrm{d}}$. Then, the discount factor of the present price becomes deterministic, namely $B_{0}^{\mathrm{d}}(T)$, and the form of the discounted payoff simplifies considerably (see for instance equation (2.21)). With this approach, we can easily apply the two-price theory with respect to the forward measure $P_{T}^{\mathrm{d}}$. In this work, we use a Weighted Value at Risk acceptability index $\alpha^{W}$ that is constructed by a proper family $\left(\Psi_{x}\right)_{x \geq 0}$ of distortion functions.

\subsection{Calibration Procedure}

We will calibrate our term structure model to market data. Therefore, a calibration procedure that deals with the two-price multiple-curve setting is presented.

Let $\Pi_{\text {ask }}^{\mathrm{mkt}}(\eta), \Pi_{\text {mid }}^{\mathrm{mkt}}(\eta)$ and $\Pi_{\mathrm{bid}}^{\mathrm{mkt}}(\eta)$ be given ask, mid and bid market prices of the 
considered derivative. The factor $\eta \in H$ denotes a certain contractual feature for a finite set $H$. For instance, $H$ could be the set of all maturities and strike rates:

$$
H=\{(T, K): T \in\{1,2, \ldots, 10\}, K \in\{0.0175,0.02,0.025,0.03,0.04, \ldots, 0.1\}\} .
$$

We postulate a multiple-curve interest rate model in a two-price economy. Their ask, mid and bid model prices are referred to as $\Pi_{\text {ask }}^{\text {mll }}(\theta, \eta, \gamma), \Pi_{\text {mid }}^{\text {mdl }}(\theta, \eta)$ and $\Pi_{\text {bid }}^{\text {mld }}(\theta, \eta, \gamma)$. We denote by $\Theta$ the set of admissible model parameters and $\theta \in \Theta$. It is assumed that at least the mid prices can be numerically evaluated fast and thus allow to calibrate the model to (mid) market data. In this thesis, we apply the Fourier based valuation method. To this end, the R-package 'Pracma' created by Borchers [87] is very useful to compute the relevant integrals.

The parameter $\gamma \geq 0$ is a fixed level of acceptability. Recall that $\gamma$ can be interpreted as the current level of market (il)liquidity. It is related to a certain discounted payoff of a derivative with a given maturity. Observe that it should hold

$$
\Pi_{\text {ask }}^{\mathrm{mdl}}(\theta, \eta, 0)=\Pi_{\text {mid }}^{\mathrm{mdl}}(\theta, \eta)=\Pi_{\mathrm{bid}}^{\mathrm{mdl}}(\theta, \eta, 0) .
$$

Our calibration procedure is made in two steps. The reason to proceed in this manner is that the numerical evaluations of the ask and bid price formulas are extremely timeconsuming in general. In our approach, we only need to calibrate the parameter $\gamma$ to the ask and bid prices. The other parameters are fitted in the first step.

1. First, we minimise the term

$$
O_{1}(\theta, H):=\sum_{\eta \in H}\left(\Pi_{\text {mid }}^{\mathrm{mdl}}(\theta, \eta)-\Pi_{\text {mid }}^{\mathrm{mkt}}(\eta)\right)^{2}
$$

over all admissible model parameters $\Theta$. Let us denote by $\hat{\theta}$ the parameter such that

$$
\begin{aligned}
O_{1}(\hat{\theta}, H) & =\sum_{\eta \in H}\left(\Pi^{\mathrm{mdl}}(\hat{\theta}, \eta)-\Pi_{\text {mid }}^{\mathrm{mkt}}(\eta)\right)^{2} \\
& =\min _{\theta \in \Theta} \sum_{\eta \in H}\left(\Pi_{\text {mid }}^{\mathrm{mdl}}(\theta, \eta)-\Pi_{\text {mid }}^{\mathrm{mkt}}(\eta)\right)^{2} .
\end{aligned}
$$

2. In the second step, we determine the parameter $\hat{\gamma} \geq 0$ satisfying

$$
O_{2}\left(\hat{\gamma}, \hat{\theta}, \bar{H}_{T}\right)=\min _{\gamma \geq 0} O_{2}\left(\gamma, \hat{\theta}, \bar{H}_{T}\right),
$$


2. Two-Price Theory in Multiple-Curve Term Structure Models

where $T$ is the corresponding maturity of the contract, $\bar{H}_{T} \subset H$ and

$$
O_{2}(\gamma, \theta, H):=\sum_{\eta \in H}\left[\left(\Pi_{\mathrm{ask}}^{\mathrm{mdl}}(\theta, \eta, \gamma)-\Pi_{\mathrm{ask}}^{\mathrm{mkt}}(\eta)\right)^{2}+\left(\Pi_{\mathrm{bid}}^{\mathrm{mdl}}(\theta, \eta, \gamma)-\Pi_{\mathrm{bid}}^{\mathrm{mkt}}(\eta)\right)^{2}\right]
$$

The resulting factors $\hat{\theta}$ and $\hat{\gamma}$ are the calibrated parameters where $\hat{\gamma}$ is related to a certain (distorted) pricing measure and the maturity $T$ (see the explanations in section 2.1). We use a modified Powell method for the minimising problems 2.24) and 2.25) (cf. Powell [78]). We stress that we just obtain local solutions for the minimising problems by our method. 


\section{THE MULTIPLE-CURVE LÉVY FORWARD RATE MODEL WITH APPLICATION OF THE TWO-PRICE THEORY}

In this chapter, we present the multiple-curve Lévy forward rate model. The Lévy forward rate model was originally introduced by Eberlein and Raible [38] and extended to timeinhomogeneous Lévy processes by Eberlein, Jacod, and Raible [39] and Eberlein and Kluge [33]. Our multiple-curve extension is based on the work of Crépey, Grbac, and Nguyen [23]. We also take into account the monotonicity of the curves. A tractable model framework that includes this fact is specified. We emphasise that the interest rates are permitted to become negative in accordance with the current market situation. Moreover, valuation formulas of interest rate derivatives with an application of the twoprice theory are stated.

\subsection{The Multiple-Curve Lévy Forward Rate Model}

Let us consider a finite time horizon $T^{*} \in \mathbb{R}_{+}, d \in \mathbb{N}^{*}=\{1,2,3, \ldots\}$ and a complete stochastic basis $\hat{\mathscr{B}}:=\left(\hat{\Omega}, \hat{\mathscr{F}}, \hat{\mathbb{F}}=\left(\hat{\mathscr{F}}_{t}\right)_{t \in\left[0, T^{*}\right]}, \hat{P}^{\mathrm{d}}\right)$. As driving process of the model we assume a $d$-dimensional time-inhomogeneous Lévy process $L=\left(L^{1}, \ldots, L^{d}\right)^{\top}$. In the multiple-curve setting, we consider a discount curve 0 (or d, respectively) and $m$ different term structures of interest rates, where we have $m \in \mathbb{N}=\{0,1,2,3, \ldots\}$ (see section 2.2. Recall that this framework contains the single-curve approach that was used before the global financial crisis by setting $m=0$ and the term structures are represented by the bond curves.

\subsubsection{Discount Curve}

Let us begin with the specification of the discount curve. To this end, we consider an arbitrary equidistant discrete tenor structure $\mathscr{T}^{\mathrm{d}}:=\left\{T_{0}, \ldots, T_{n}\right\}$. Recall that this means 
that we have $n \in \mathbb{N}^{*}, T_{0}<\cdots<T_{n}$ and $T_{n}=T^{*}$. We denote by $\delta:=\delta\left(T_{k-1}, T_{k}\right)$ the year fraction between the dates $T_{k-1}$ and $T_{k}$. As usual, it is called the tenor of $\mathscr{T}^{\mathrm{d}}$. The time- $t$ risk-free zero-coupon bond price maturing at date $T \in\left[0, T^{*}\right]$ is denoted by $B_{t}^{\mathrm{d}}(T)$ (discount bond). We denote by $\mathscr{P}$ the predictable $\sigma$-field on $\hat{\Omega} \times\left[0, T^{*}\right]$ which is defined as the $\sigma$-field on $\hat{\Omega} \times\left[0, T^{*}\right]$ generated by all adapted processes that are left-continuous and considered as mappings on $\hat{\Omega} \times\left[0, T^{*}\right]$ (cf. Jacod and Shiryaev [56, Definition I.2.1]). The optional $\sigma$-field $\mathscr{O}$ on $\hat{\Omega} \times\left[0, T^{*}\right]$ is defined as the $\sigma$-field on $\hat{\Omega} \times\left[0, T^{*}\right]$ which is generated by all càdlàg adapted processes considered as mappings on $\hat{\Omega} \times\left[0, T^{*}\right]$ (cf. Jacod and Shiryaev [56, Definition I.1.20]).

The following additional ingredients are needed to develop the model for the discount curve:

$(\mathbb{D} .1)$ The initial discount curve $B_{0}^{\text {d }}$ defined by

$$
B_{0}^{\mathrm{d}}:\left\{\begin{array}{l}
{\left[0, T^{*}\right] \rightarrow(0, \infty)} \\
T \mapsto B_{0}^{\mathrm{d}}(T)
\end{array}\right.
$$

is given.

We assume that the initial discount curve is built by using an appropriate bootstrapping technique. A general explanation of a bootstrapping method is given by Hull [55] and Ametrano and Bianchetti [3]. One typically takes the OIS-zero-coupon bond as an approximation of $B_{0}^{\mathrm{d}}$.

$(\mathbb{D} .2)$ We consider a drift function $\alpha^{\mathrm{d}}$ and a volatility structure $\sigma^{\mathrm{d}}$ defined by

$$
\alpha^{\mathrm{d}}:\left\{\begin{array}{l}
\hat{\Omega} \times\left[0, T^{*}\right] \times\left[0, T^{*}\right] \rightarrow \mathbb{R} \\
(\hat{\omega}, s, T) \mapsto \alpha^{\mathrm{d}}(\hat{\omega}, s, T)
\end{array}\right.
$$

and

$$
\sigma^{\mathrm{d}}:\left\{\begin{array}{l}
\hat{\Omega} \times\left[0, T^{*}\right] \times\left[0, T^{*}\right] \rightarrow \mathbb{R}^{d} \\
(\hat{\omega}, s, T) \mapsto \sigma^{\mathrm{d}}(\hat{\omega}, s, T)=\left(\sigma_{1}^{\mathrm{d}}(\hat{\omega}, s, T), \ldots, \sigma_{d}^{\mathrm{d}}(\hat{\omega}, s, T)\right)
\end{array}\right.
$$

which satisfy the usual measurability and boundedness conditions (cf. Eberlein, Jacod, and Raible [39]):

(i) $\alpha^{\mathrm{d}}$ and $\sigma^{\mathrm{d}}=\left(\sigma_{1}^{\mathrm{d}}, \ldots, \sigma_{d}^{\mathrm{d}}\right)$ are measurable with respect to $\mathscr{P} \otimes \mathcal{B}\left(\left[0, T^{*}\right]\right)$.

(ii) The random functions are bounded for each $\hat{\omega} \in \hat{\Omega}$ in the sense of

$$
\sup _{0 \leq s, T \leq T^{*}}\left(\left|\alpha^{\mathrm{d}}(\hat{\omega}, s, T)\right|+\left\|\sigma^{\mathrm{d}}(\hat{\omega}, s, T)\right\|\right)<\infty
$$

where $\|\cdot\|$ denotes the Euclidean norm here. 
(iii) For every $(\hat{\omega}, s, T) \in \hat{\Omega} \times\left[0, T^{*}\right] \times\left[0, T^{*}\right]$ with $T<s$, we have $\alpha^{\mathrm{d}}(\hat{\omega}, s, T)=0$ and $\sigma^{\mathrm{d}}(\hat{\omega}, s, T)=(0, \ldots, 0)$.

Let us postulate that, for every fixed maturity $T \in\left[0, T^{*}\right]$, the dynamics of the discount instantaneous forward rates $f^{\mathrm{d}}(T)=\left(f_{t}^{\mathrm{d}}(T)\right)_{t \in[0, T]}$ are modelled as

$$
f_{t}^{\mathrm{d}}(T)=f_{0}^{\mathrm{d}}(T)+\int_{0}^{t} \alpha^{\mathrm{d}}(s, T) d s-\int_{0}^{t} \sigma^{\mathrm{d}}(s, T) d L_{s} .
$$

The initial values $f_{0}^{\mathrm{d}}(T)$ are assumed to form a deterministic, bounded and $\left(\mathcal{B}\left(\left[0, T^{*}\right]\right), \mathcal{B}(\mathbb{R})\right)$ measurable mapping

$$
\left[0, T^{*}\right] \ni T \mapsto f_{0}^{\mathrm{d}}(T) \in \mathbb{R} .
$$

It is shown by Eberlein, Jacod, and Raible [39] that under these assumptions we can find a joint-version of all $f_{t}^{\mathrm{d}}(T)$ such that the map $(\hat{\omega}, t, T) \mapsto f_{t}^{\mathrm{d}}(\hat{\omega}, T) \mathbb{1}_{\{t \leq T\}}$ is $\mathscr{O} \otimes \mathcal{B}\left(\left[0, T^{*}\right]\right)$-measurable. Consequently, we can build an integral with the forward rate as integrand. It follows from the forward rate dynamics $(3.1)$ and an application of Fubini's Theorem (cf. Protter [79, Theorem 64]) that the bond price $B_{t}^{\mathrm{d}}(T)$ at time $t$ given by

$$
B_{t}^{\mathrm{d}}(T)=\exp \left(-\int_{t}^{T} f_{t}^{\mathrm{d}}(u) d u\right)
$$

can be expressed as

$$
B_{t}^{\mathrm{d}}(T)=B_{0}^{\mathrm{d}}(T) \exp \left(\int_{0}^{t}\left(r_{s}^{\mathrm{d}}-A^{\mathrm{d}}(s, T)\right) d s+\int_{0}^{t} \Sigma^{\mathrm{d}}(s, T) d L_{s}\right),
$$

where the short rate $r_{t}^{\mathrm{d}}$ at time $t$ is specified by $r_{t}^{\mathrm{d}}=f_{t}^{\mathrm{d}}(t)$ and we set

$$
A^{\mathrm{d}}(s, T):=\int_{s \wedge T}^{T} \alpha^{\mathrm{d}}(s, u) d u \quad \text { and } \quad \Sigma^{\mathrm{d}}(s, T):=\int_{s \wedge T}^{T} \sigma^{\mathrm{d}}(s, u) d u
$$

(cf. Eberlein and Kluge [33]). Note that the integral $\int_{s \wedge T}^{T} \sigma^{\mathrm{d}}(s, u) d u$ is clearly understood componentwise and the initial values $f_{0}^{\mathrm{d}}(T)$ can be obtained by the relation

$$
f_{0}^{\mathrm{d}}(T)=-\frac{\partial \ln B_{0}^{\mathrm{d}}(T)}{\partial T}
$$

if the derivative exists. 
The discount factor process $\beta^{\mathrm{d}}=\left(\beta_{t}^{\mathrm{d}}\right)_{t \in\left[0, T^{*}\right]}$ given by

$$
\beta_{t}^{\mathrm{d}}=\exp \left(-\int_{0}^{t} r_{s}^{\mathrm{d}} d s\right)
$$

is an adapted process with continuous paths. It can obviously be written as

$$
\beta_{t}^{\mathrm{d}}=B_{0}^{\mathrm{d}}(t) \exp \left(-\int_{0}^{t} A^{\mathrm{d}}(s, t) d s+\int_{0}^{t} \Sigma^{\mathrm{d}}(s, t) d L_{s}\right) .
$$

From 3.3 together with 3.5 , one easily verifies the useful representation

$$
B_{t}^{\mathrm{d}}(T)=\frac{B_{0}^{\mathrm{d}}(T)}{B_{0}^{\mathrm{d}}(t)} \exp \left(\int_{0}^{t}\left(A^{\mathrm{d}}(s, t)-A^{\mathrm{d}}(s, T)\right) d s+\int_{0}^{t} \Sigma^{\mathrm{d}}(s, t, T) d L_{s}\right),
$$

where we set $\Sigma^{\mathrm{d}}(s, t, T):=\Sigma^{\mathrm{d}}(s, T)-\Sigma^{\mathrm{d}}(s, t)$. To get a tractable model and guarantee the existence of all related functions, we additionally require the model to be based on the standing assumption of a deterministic, bounded and continuous volatility structure:

Assumption $(\mathbb{D E T}): \quad$ The volatility structure $\sigma^{\mathrm{d}}$ is a deterministic and bounded function such that, for every $s$ and $T$ with $0 \leq s, T \leq T^{*}$, it holds

$$
0 \leq \Sigma_{j}^{\mathrm{d}}(s, T) \leq \hat{M}<M, \quad \text { for every } j \in\{1, \ldots, d\},
$$

where $\Sigma_{j}^{\mathrm{d}}$ arises from definition 3.4 ) ( $j^{\text {th }}$ component of $\Sigma^{\mathrm{d}}$ ) and the constant $M$ is from assumption $(\mathbb{E M})$. Moreover, the mapping

$$
\left[0, T^{*}\right] \ni s \mapsto \sigma^{\mathrm{d}}(s, T) \in \mathbb{R}^{d}
$$

is continuous for each given $T \in\left[0, T^{*}\right]$.

Observe that we have $\sigma^{\mathrm{d}}(s, s)=0$ for every $s \in\left[0, T^{*}\right]$ under this assumption. Let $\mathscr{M}^{\mathrm{d}}:=\left(\left\{B^{\mathrm{d}}\left(T_{0}\right), \ldots, B^{\mathrm{d}}\left(T_{n}\right)\right\}, \mathcal{S}\right)$ be a financial market and $\mathcal{S}$ denote the space of admissible trading strategies. By the fundamental Theorem of asset pricing (see Delbaen and Schachermayer [25]), the existence of a local martingale measure is a sufficient criterion that ensures the absence of arbitrage in $\mathscr{M}^{\mathrm{d}}$. More specifically, this means that there exists a probability measure under which the discounted zero coupon bond price processes are local martingales. Eberlein and Kluge [33, Proposition 9 and the preliminary remarks] derive a condition on the drift function $A^{\mathrm{d}}$ such that for all $T \in\left[0, T^{*}\right]$, and therefore for all $T \in \mathscr{T}^{\mathrm{d}}$, the discounted bond price process $Z^{\mathrm{d}}(T)=\left(Z_{t}^{\mathrm{d}}(T)\right)_{0 \leq t \leq T}$ given by

$$
Z_{t}^{\mathrm{d}}(T):=\beta_{t}^{\mathrm{d}} B_{t}^{\mathrm{d}}(T)
$$


is an $\left(\hat{\mathbb{F}}, \hat{P}^{\mathrm{d}}\right)$-martingale. Here, the result of Eberlein and Raible [38, Lemma 3.1] and its generalised version given by Kluge [64, Proposition 1.9] play a crucial role: We can determine the term

$$
\mathbb{E}_{\hat{P}^{\mathrm{d}}}\left[\exp \left(\int_{0}^{t} \Sigma^{\mathrm{d}}(s, T) d L_{s}\right)\right]
$$

in the form of the cumulant function $\theta_{t}$ of $L$ with respect to measure $\hat{P}^{\mathrm{d}}$, namely

$$
\mathbb{E}_{\hat{P}^{\mathrm{d}}}\left[\exp \left(\int_{0}^{t} \Sigma^{\mathrm{d}}(s, T) d L_{s}\right)\right]=\exp \left(\int_{0}^{t} \theta_{s}\left(\Sigma^{\mathrm{d}}(s, T)\right) d s\right) .
$$

They obtain the following generalisation of the famous Heath-Jarrow-Morton no-arbitrage condition that will be a standing assumption in this model:

Assumption $(\mathbb{N} A)$ : The function $A^{\mathrm{d}}$ is given by the form

$$
A^{\mathrm{d}}(s, T)=\theta_{s}\left(\Sigma^{\mathrm{d}}(s, T)\right) .
$$

Notice that this approach makes the Lévy forward rate model of Eberlein and Kluge [33] related to the discount curve work directly under the risk-neutral measure. This choice of the drift term is closely related to the notion of the exponential compensator (see Kallsen and Shiryaev [61] and Jacod and Shiryaev [56, Section II.8]). We emphasise that the drift function $A^{\mathrm{d}}$ and therefore also $\alpha^{\mathrm{d}}$ as well as the volatility structure $\Sigma^{\mathrm{d}}$ (and $\sigma^{\mathrm{d}}$ ) are deterministic in this setting.

Lemma 3.1.1 For $t, T \in\left[0, T^{*}\right]$ with $t \leq T$, the discount forward rate $f_{t}^{\mathrm{d}}(T)$ is given in terms of

$$
f_{t}^{\mathrm{d}}(T)=f_{0}^{\mathrm{d}}(T)+\int_{0}^{t} \frac{\partial}{\partial T} \theta_{s}\left(\Sigma^{\mathrm{d}}(s, T)\right) d s-\int_{0}^{t} \sigma^{\mathrm{d}}(s, T) d L_{s}
$$

and the short rate $r_{t}^{\mathrm{d}}$ has the representation

$$
r_{t}^{\mathrm{d}}=f_{0}^{\mathrm{d}}(t)+\int_{0}^{t} \frac{\partial}{\partial t} \theta_{s}\left(\Sigma^{\mathrm{d}}(s, t)\right) d s-\int_{0}^{t} \sigma^{\mathrm{d}}(s, t) d L_{s}
$$

Proof: The statements (3.8) and (3.9) are immediately clear on the basis of the relation

$$
\alpha^{\mathrm{d}}(s, T)=\frac{\partial}{\partial T} \theta_{s}\left(\Sigma^{\mathrm{d}}(s, T)\right)
$$

together with $(3.1)$, relation $(3.4)$, assumption $(\mathbb{N} A)$ and the definition of the short rate.

For some important comments on the (partial) derivative of the cumulant process we 
refer to Kallsen and Shiryaev [61, section 2.5].

Under assumption $(\mathbb{N} A)$, the bond price $(3.3)$ is given in the more specific form

$$
B_{t}^{\mathrm{d}}(T)=\frac{B_{0}^{\mathrm{d}}(T)}{\beta_{t}^{\mathrm{d}}} \exp \left(-\int_{0}^{t} \theta_{s}\left(\Sigma^{\mathrm{d}}(s, T)\right) d s+\int_{0}^{t} \Sigma^{\mathrm{d}}(s, T) d L_{s}\right)
$$

from which we conclude that

$$
\frac{Z_{t}^{\mathrm{d}}(T)}{Z_{0}^{\mathrm{d}}(T)}=\exp \left(-\int_{0}^{t} \theta_{s}\left(\Sigma^{\mathrm{d}}(s, T)\right) d s+\int_{0}^{t} \Sigma^{\mathrm{d}}(s, T) d L_{s}\right) .
$$

Lemma 3.1.2 In this model framework, the discounted bond price process $Z^{\mathrm{d}}(T)$ is the solution of

$$
d Z^{\mathrm{d}}(T)=Z_{-}^{\mathrm{d}}(T) d \bar{Y}^{\mathrm{d}}
$$

where the process $\bar{Y}^{\mathrm{d}}$ is given by

$$
\bar{Y}_{t}^{\mathrm{d}}=\int_{0}^{t} \Sigma^{\mathrm{d}}(s, T) \sqrt{c_{s}} d W_{s}+\int_{0}^{t} \int_{\mathbb{R}^{d}}\left(e^{\left\langle\Sigma^{\mathrm{d}}(s, T), x\right\rangle}-1\right)\left(\mu^{L}-\nu\right)(d s, d x) .
$$

Proof: We apply Jacod and Shiryaev [56, Theorem II.8.10] with equation 3.10 to get

$$
\frac{Z_{t}^{\mathrm{d}}(T)}{Z_{0}^{\mathrm{d}}(T)}=\mathscr{E}_{t}\left(\bar{Y}^{\mathrm{d}}\right)
$$

where the process $\bar{Y}^{\mathrm{d}}$ is specified by the stochastic logarithm $\bar{Y}^{\mathrm{d}}=\mathscr{L}\left(\exp \left(Y^{\mathrm{d}}\right)\right)$ with

$$
Y_{t}^{\mathrm{d}}=-\int_{0}^{t} \theta_{s}\left(\Sigma^{\mathrm{d}}(s, T)\right) d s+\int_{0}^{t} \Sigma^{\mathrm{d}}(s, T) d L_{s}
$$

Then, we immediately obtain the statement.

This Lemma confirms once again that $Z^{\mathrm{d}}(T)$ is a $\hat{P}^{\mathrm{d}}$-(local) martingale (cf. Kallsen and Shiryaev [61]). To end this subsection, we introduce some useful definitions. We observe that representation (3.6) results in

$$
\begin{aligned}
B_{t}^{\mathrm{d}}(T)=\frac{B_{0}^{\mathrm{d}}(T)}{B_{0}^{\mathrm{d}}(t)} \exp ( & \int_{0}^{t}\left[\theta_{s}\left(\Sigma^{\mathrm{d}}(s, t)\right)-\theta_{s}\left(\Sigma^{\mathrm{d}}(s, T)\right)\right] d s \\
+ & \left.\int_{0}^{t} \Sigma^{\mathrm{d}}(s, t, T) d L_{s}\right) .
\end{aligned}
$$

For fixed $t, T \in\left[0, T^{*}\right]$ with $t \leq T$, the last expression is decomposed into its deterministic 
part defined by

$$
D^{\mathrm{d}}(t, T):=\frac{B_{0}^{\mathrm{d}}(T)}{B_{0}^{\mathrm{d}}(t)} \exp \left(\int_{0}^{t}\left[\theta_{s}\left(\Sigma^{\mathrm{d}}(s, t)\right)-\theta_{s}\left(\Sigma^{\mathrm{d}}(s, T)\right)\right] d s\right)
$$

and its stochastic part given as the exponential of the $\hat{\mathscr{F}}_{t}$-measurable random variable

$$
X^{\mathrm{d}}(t, T):=\int_{0}^{t} \Sigma^{\mathrm{d}}(s, t, T) d L_{s} .
$$

Hence, we obtain the compact form

$$
B_{t}^{\mathrm{d}}(T)=D^{\mathrm{d}}(t, T) \exp \left(X^{\mathrm{d}}(t, T)\right) .
$$

\subsubsection{Multiple Curves}

Now we address the modelling of the risky curves. Let us consider $m \in \mathbb{N}^{*}$ different curves. Since each term structure corresponds to a discrete tenor structure, we introduce the equidistant tenor structure $\mathscr{T}^{k}:=\left\{T_{0}^{k}, \ldots, T_{n_{k}}^{k}\right\}$ for every $k \in\{1, \ldots, m\}$ and $n_{k} \in \mathbb{N}$. We assume that it holds $T_{0}^{k}=T_{0}$ and $T_{n_{k}}^{k}=T_{n}=T^{*}$ for all $k \in\{1, \ldots, m\}$. The year fraction between the dates $T_{j-1}^{k}$ and $T_{j}^{k}$ is denoted by $\delta^{k}:=\delta^{k}\left(T_{j-1}^{k}, T_{j}^{k}\right)$, where $j \in\left\{1, \ldots, n_{k}\right\}$. Note that $\delta^{k}$ unambiguously corresponds to the tenor structure $\mathscr{T}^{k}$ and we can therefore call $\delta^{k}$ the tenor of $\mathscr{T}^{k}$. Moreover, for all $k, l \in\{1, \ldots, m\}$ with $k \leq l$, we postulate

$$
\mathscr{T}^{l} \subset \mathscr{T}^{k} \subset \mathscr{T}^{\mathrm{d}} \subset\left[0, T^{*}\right] .
$$

In accordance with the multiple curve approach, this assumption means that the curves reflect liquidity and credit risk in decreasing order of magnitude $\left(\delta^{k}<\delta^{l}\right)$.

For every $k \in\{1, \ldots, m\}$, we interpret $B_{t}^{k}(T)$ as time- $t$ price of a fictitious risky zerocoupon bond with maturity $T$ that corresponds to curve $k$. Notice that such risky bonds are not traded assets but can be considered as bonds being issued by an average Libor or Euribor panel member.

We need some additional ingredients to model the multiple curves:

(MC.1) The initial multiple term structure curves $B_{0}^{1}, \ldots, B_{0}^{m}$ defined for every $k \in\{1, \ldots, m\}$ by

$$
B_{0}^{k}:\left\{\begin{array}{l}
{\left[0, T^{*}\right] \rightarrow(0, \infty)} \\
T \mapsto B_{0}^{k}(T)
\end{array}\right.
$$

are given. 
Ametrano and Bianchetti [2, 3] developed a sophisticated bootstrapping method dealing with this multiple-curve setting. Note that, by using this bootstrapping procedure, the initial values satisfy

$$
B_{0}^{l}(T) \leq B_{0}^{k}(T) \leq B_{0}^{\mathrm{d}}(T)
$$

for every $k, l \in\{1, \ldots, m\}$ with $k \leq l$ and $T \in\left[0, T^{*}\right]$.

(MC.2) For every $k \in\{1, \ldots, m\}$, we consider the drift function $\alpha^{k}$ and the volatility structure $\sigma^{k}$ defined by

$$
\alpha^{k}:\left\{\begin{array}{l}
\hat{\Omega} \times\left[0, T^{*}\right] \times\left[0, T^{*}\right] \rightarrow \mathbb{R} \\
(\hat{\omega}, s, T) \mapsto \alpha^{k}(\hat{\omega}, s, T)
\end{array}\right.
$$

and

$$
\sigma^{k}:\left\{\begin{array}{l}
\hat{\Omega} \times\left[0, T^{*}\right] \times\left[0, T^{*}\right] \rightarrow \mathbb{R}^{d} \\
(\hat{\omega}, s, T) \mapsto \sigma^{k}(\hat{\omega}, s, T)=\left(\sigma_{1}^{k}(\hat{\omega}, s, T), \ldots, \sigma_{d}^{k}(\hat{\omega}, s, T)\right)
\end{array}\right.
$$

which satisfy the same (measurability and boundedness) conditions as $\alpha^{\mathrm{d}}$ and $\sigma^{\mathrm{d}}$ in $(\mathbb{D} \cdot 2)$.

For every $k \in\{1, \ldots, m\}$ and $T \in\left[0, T^{*}\right]$, the dynamics of the instantaneous forward rates $f^{k}(T)=\left(f_{t}^{k}(T)\right)_{t \in[0, T]}$ are postulated to be

$$
f_{t}^{k}(T)=f_{0}^{k}(T)+\int_{0}^{t} \alpha^{k}(s, T) d s-\int_{0}^{t} \sigma^{k}(s, T) d L_{s},
$$

where the initial values $f_{0}^{k}(T)$ are assumed to be deterministic, bounded and $\left(\mathcal{B}\left(\left[0, T^{*}\right]\right), \mathcal{B}(\mathbb{R})\right)$ measurable in $T$. It can be determined by the formula

$$
f_{0}^{k}(T)=-\frac{\partial \ln B_{0}^{k}(T)}{\partial T}
$$

if the derivative exists. In the same way as one gets representation $(3.3)$, we obtain the form

$$
B_{t}^{k}(T)=B_{0}^{k}(T) \exp \left(\int_{0}^{t}\left(r_{s}^{k}-A^{k}(s, T)\right) d s+\int_{0}^{t} \Sigma^{k}(s, T) d L_{s}\right)
$$

for each $k \in\{1, \ldots, m\}$ from the relation

$$
B_{t}^{k}(T)=\exp \left(-\int_{t}^{T} f_{t}^{k}(u) d u\right)
$$


The rate $r_{t}^{k}$ at $t$ is given by $r_{t}^{k}=f_{t}^{k}(t)$ and we similarly define

$$
A^{k}(s, T):=\int_{s \wedge T}^{T} \alpha^{k}(s, u) d u \quad \text { and } \quad \Sigma^{k}(s, T):=\int_{s \wedge T}^{T} \sigma^{k}(s, u) d u .
$$

To ensure the existence of the cumulant process and for practical purposes, we need the following standing assumption:

Assumption $(\mathbb{M C} . \mathbb{D E} \mathbb{T}):$ For any $k \in\{1, \ldots, m\}$ and all $s, T \in\left[0, T^{*}\right]$, it holds that the volatility structure $\sigma^{k}$ is deterministic and bounded in the sense of

$$
0 \leq \Sigma_{j}^{k}(s, T) \leq \hat{M}<M, \text { for every } j \in\{1, \ldots, d\} .
$$

The mapping $\left[0, T^{*}\right] \ni s \mapsto \sigma^{k}(s, T) \in \mathbb{R}^{d}$ is continuous. As usual, $M$ is the constant from assumption $(\mathbb{E M})$.

Note that the constant $\hat{M}$ in this assumption does not have to coincide with the constant $\hat{M}$ from assumption $(\mathbb{D E T})$. The discounted bond price process $Z^{k}(T)=$ $\left(Z_{t}^{k}(T)\right)_{0 \leq t \leq T}$ corresponding to curve $k$ is defined by

$$
Z_{t}^{k}(T):=\beta_{t}^{\mathrm{d}} B_{t}^{k}(T)
$$

for each date $T \in\left[0, T^{*}\right]$. One easily verifies that

$$
\frac{Z_{t}^{k}(T)}{Z_{0}^{k}(T)}=\exp \left(\int_{0}^{t}\left[r_{s}^{k}-r_{s}^{\mathrm{d}}-A^{k}(s, T)\right] d s+\int_{0}^{t} \Sigma^{k}(s, T) d L_{s}\right)
$$

Lemma 3.1.3 The discounted bond price process $Z^{k}(T)$ is the solution of

$$
d Z^{k}(T)=Z_{-}^{k}(T) d \bar{Y}^{k}
$$

where the process $\bar{Y}^{k}=\left(\bar{Y}_{t}^{k}\right)_{0 \leq t \leq T}$ is given by

$$
\begin{aligned}
\bar{Y}_{t}^{k}= & \int_{0}^{t}\left[r_{s}^{k}-r_{s}^{\mathrm{d}}-A^{k}(s, T)+\theta_{s}\left(\Sigma^{k}(s, T)\right)\right] d s+\int_{0}^{t} \Sigma^{k}(s, T) \sqrt{c_{s}} d W_{s} \\
& +\int_{0}^{t} \int_{\mathbb{R}^{d}}\left(e^{\left\langle\Sigma^{k}(s, T), x\right\rangle}-1\right)\left(\mu^{L}-\nu\right)(d s, d x) .
\end{aligned}
$$

Proof: We apply Jacod and Shiryaev [56, Theorem II.8.10] with relation (3.17) and obtain

$$
\frac{Z_{t}^{k}(T)}{Z_{0}^{k}(T)}=\mathscr{E}_{t}\left(\bar{Y}^{k}\right)
$$


where the process $\bar{Y}^{k}$ is set as the stochastic logarithm $\bar{Y}^{k}=\mathscr{L}\left(\exp \left(Y^{k}\right)\right)$ with

$$
Y_{t}^{k}=\int_{0}^{t}\left(r_{s}^{k}-r_{s}^{\mathrm{d}}-A^{k}(s, T)\right) d s+\int_{0}^{t} \Sigma^{k}(s, T) d L_{s}
$$

We get the form

$$
\begin{aligned}
\bar{Y}_{t}^{k}= & \int_{0}^{t}\left[r_{s}^{k}-r_{s}^{\mathrm{d}}-A^{k}(s, T)+\left\langle\Sigma^{k}(s, T), b_{s}\right\rangle+\frac{1}{2}\left\langle\Sigma^{k}(s, T), c_{s} \Sigma^{k}(s, T)\right\rangle\right. \\
& \left.+\int_{\mathbb{R}^{d}}\left(e^{\left\langle\Sigma^{k}(s, T), x\right\rangle}-1-\left\langle\Sigma^{k}(s, T), x\right\rangle\right) F_{s}(d x)\right] d s \\
& +\int_{0}^{t} \Sigma^{k}(s, T) \sqrt{c_{s}} d W_{s}+\int_{0}^{t} \int_{\mathbb{R}^{d}}\left(e^{\left\langle\Sigma^{k}(s, T), x\right\rangle}-1\right)\left(\mu^{L}-\nu\right)(d s, d x) \\
= & \int_{0}^{t}\left[r_{s}^{k}-r_{s}^{\mathrm{d}}-A^{k}(s, T)+\theta_{s}\left(\Sigma^{k}(s, T)\right)\right] d s+\int_{0}^{t} \Sigma^{k}(s, T) \sqrt{c_{s}} d W_{s} \\
& +\int_{0}^{t} \int_{\mathbb{R}^{d}}\left(e^{\left\langle\Sigma^{k}(s, T), x\right\rangle}-1\right)\left(\mu^{L}-\nu\right)(d s, d x) .
\end{aligned}
$$

This Lemma shows that $Z^{k}(T)$ is not a $\hat{P}^{\mathrm{d}}$-(local) martingale in general.

Similar to relation (3.11), we rewrite the expression (3.15) as

$$
B_{t}^{k}(T)=\frac{B_{0}^{k}(T)}{B_{0}^{k}(t)} \exp \left(\int_{0}^{t}\left[A^{k}(s, t)-A^{k}(s, T)\right] d s+\int_{0}^{t} \Sigma^{k}(s, t, T) d L_{s}\right),
$$

where we analogously set

$$
\Sigma^{k}(s, t, T):=\Sigma^{k}(s, T)-\Sigma^{k}(s, t) .
$$

To simplify the notation, for fixed $t, T \in\left[0, T^{*}\right]$ with $t \leq T$, we define the factor

$$
D^{k}(t, T):=\frac{B_{0}^{k}(T)}{B_{0}^{k}(t)} \exp \left(\int_{0}^{t}\left[A^{k}(s, t)-A^{k}(s, T)\right] d s\right)
$$

and the $\hat{\mathscr{F}}_{\text {- }}$-measurable random variable

$$
X^{k}(t, T):=\int_{0}^{t} \Sigma^{k}(s, t, T) d L_{s} .
$$

Then, we obtain

$$
B_{t}^{k}(T)=D^{k}(t, T) \exp \left(X^{k}(t, T)\right) .
$$

Note that at this stage it is not clear if $D^{k}$ can be chosen deterministically. 
Now, we specify the drift function $A^{k}$ in such a manner that credit and liquidity risk issues are taken into account. We closely follow the approach of Crépey, Grbac, and Nguyen [23, Section 2.3.2]. Their idea is based on no-arbitrage requirements in defaultable HJM-models which lead to the required drift conditions. Let us temporarily assume that defaultable bonds with respect to each curve can be traded in the market. The time- $t$ price of such a bond maturing at $T$ is denoted by $\bar{B}_{t}^{k}(T)$. We then study the conditions that preclude arbitrage opportunities by dealing with these bonds (cf. Delbaen and Schachermayer [25]). Keep in mind that such bonds are actually not traded in the market (see also the comments in Crépey, Grbac, and Nguyen [23, section 2.3.2]). In fact, they are rather mathematical concepts which represent the credit risk of the panel bank members and are not defaultable in the classical sense.

To develop an appropriate credit risk model, let us construct default times $\tau^{1}, \ldots, \tau^{m}$ as it is described in detail in section 1.2.2. To this end, we need to enlarge the initial stochastic basis $\left(\hat{\Omega}, \hat{\mathscr{F}}, \hat{\mathbb{F}}=\left(\hat{\mathscr{F}}_{t}\right)_{t \in\left[0, T^{*}\right]}, \hat{P}^{\mathrm{d}}\right)$ towards $\left(\Omega, \mathscr{G}, \mathbb{F}=\left(\mathscr{F}_{t}\right)_{t \in\left[0, T^{*}\right]}, P^{\mathrm{d}}\right)$ and $\left(\Omega, \mathscr{G}, \mathbb{G}=\left(\mathscr{G}_{t}\right)_{t \in\left[0, T^{*}\right]}, P^{\mathrm{d}}\right)$, respectively, where we used the notation for the relevant quantities as in section 1.2 .2 (except for $P^{\mathrm{d}}:=\hat{P}^{\mathrm{d}} \otimes \tilde{P}$ ). Further, we assume that the defaultable bonds pay a certain recovery upon default. This recovery payment is specified by the terminal recovery process $R^{k}=\left(R_{t}^{k}\right)_{t \in\left[0, T^{*}\right]}$ for every curve $k \in\{1, \ldots, m\}$. The process $R^{k}$ is $\hat{\mathbb{F}}$-adapted and (locally) bounded on $\left(\hat{\Omega}, \hat{\mathscr{F}}, \hat{\mathbb{F}}, \hat{P}^{\mathrm{d}}\right)$ (cf. Bielecki and Rutkowski [12, Section 13.1.9.]). In financial interpretation, the amount $R_{\tau^{k}}^{k}$ is the recovery payment made at maturity $T$ if the default of the bond issuer occurs at time $\tau^{k} \leq T$. More specifically, the value of the defaultable bond price $\bar{B}_{T}^{k}(T)$ at time $T$ is given by

$$
\begin{aligned}
\bar{B}_{T}^{k}(T) & =\mathbb{1}_{\left\{\tau^{k}>T\right\}} B_{T}^{k}(T)+B_{T}^{\mathrm{d}}(T) R_{\tau^{k}}^{k} \mathbb{1}_{\left\{\tau^{k} \leq T\right\}} \\
& =\mathbb{1}_{\left\{\tau^{k}>T\right\}}+R_{\tau^{k}}^{k} \mathbb{1}_{\left\{\tau^{k} \leq T\right\}} .
\end{aligned}
$$

Then, the time- $t$ price results in

$$
\bar{B}_{t}^{k}(T)=\mathbb{1}_{\left\{\tau^{k}>t\right\}} B_{t}^{k}(T)+B_{t}^{\mathrm{d}}(T) R_{\tau^{k}}^{k} \mathbb{1}_{\left\{\tau^{k} \leq t\right\}}
$$

and we obtain its discounted value $\bar{Z}_{t}^{k}(T):=\beta_{t}^{\mathrm{d}} \bar{B}_{t}^{k}(T)$ as

$$
\bar{Z}_{t}^{k}(T)=\mathbb{1}_{\left\{\tau^{k}>t\right\}} Z_{t}^{k}(T)+R_{\tau^{k}}^{k} \mathbb{1}_{\left\{\tau^{k} \leq t\right\}} Z_{t}^{\mathrm{d}}(T)
$$

Consequently, the time- $t$ bond price $B_{t}^{k}(T)$ is interpreted as the pre-default price of the associated defaultable zero-coupon bond. We postulate that each random time $\tau^{k}$ possesses an $\mathbb{F}$-intensity $\gamma^{k}$.

The following Theorem states the conditions which ensure the absence of arbitrage in our model. To be precise, we derive conditions such that for every $k \in\{1, \ldots, m\}$, the 
discounted defaultable bond price process $\bar{Z}^{k}(T)$ is a $\left(\mathbb{G}, P^{\mathrm{d}}\right)$-local martingale for each $T \in\left[0, T^{*}\right]$.

Theorem 3.1.1 Assume that, for each $k \in\{1, \ldots, m\}$ and $T \in\left[0, T^{*}\right]$, the condition

$$
Z_{t-}^{k}(T)\left[\lambda_{t}^{k, \mathrm{~d}}-A^{k}(t, T)+\theta_{t}\left(\Sigma^{k}(t, T)\right)\right]=\left(Z_{t-}^{k}(T)-R_{t}^{k} Z_{t}^{\mathrm{d}}(T)\right) \gamma_{t}^{k}
$$

is satisfied for all $t \in[0, T]$, where we set $\lambda_{t}^{k, \mathrm{~d}}:=r_{t}^{k}-r_{t}^{\mathrm{d}}$. Then, for each $k \in\{1, \ldots, m\}$ and $T \in\left[0, T^{*}\right]$, the process $\bar{Z}^{k}(T)$ is a $\left(\mathbb{G}, P^{\mathrm{d}}\right)$-local martingale.

Proof: For every $k \in\{1, \ldots, m\}$ and $T \in\left[0, T^{*}\right]$, we obviously have that $H^{k}=\mathbb{1}_{\left\{\tau^{k} \leq \cdot\right\}} \in$ $\mathscr{V}, 1-H^{k}=\mathbb{1}_{\left\{\tau^{k}>\cdot\right\}} \in \mathscr{V}$ and $Z^{k}(T) \in \mathscr{S}$. Let us define by $\hat{H}_{t}^{k}:=R_{\tau^{k}}^{k} H_{t}^{k}$ a $\mathbb{G}$-adapted process $\hat{H}^{k}=\left(\hat{H}_{t}^{k}\right)_{t \in\left[0, T^{*}\right]}$ in $\mathscr{V}$. Then, by Jacod and Shiryaev [56, Proposition I.4.49], we obtain that

$$
\begin{aligned}
\bar{Z}_{t}^{k}(T)= & \int_{0}^{t}\left(1-H_{s-}^{k}\right) d Z_{s}^{k}(T)+\int_{0}^{t} Z_{s}^{k}(T) d\left(1-H_{s}^{k}\right)+\bar{Z}_{0}^{k}(T) \\
& +\int_{0}^{t} \hat{H}_{s-}^{k} d Z_{s}^{\mathrm{d}}(T)+\int_{0}^{t} Z_{s}^{\mathrm{d}}(T) d \hat{H}_{s}^{k} \\
= & \int_{0}^{t}\left(1-H_{s-}^{k}\right) d Z_{s}^{k}(T)-\int_{0}^{t} Z_{s}^{k}(T) d H_{s}^{k}+\bar{Z}_{0}^{k}(T) \\
& +\int_{0}^{t} \hat{H}_{s-}^{k} d Z_{s}^{\mathrm{d}}(T)+\int_{0}^{t} R_{s}^{k} Z_{s}^{\mathrm{d}}(T) d H_{s}^{k} .
\end{aligned}
$$

Using the form 3.18 , one gets

$$
\begin{aligned}
\bar{Z}_{t}^{k}(T)= & \int_{0}^{t}\left(1-H_{s-}^{k}\right) Z_{s-}^{k}(T)\left[\lambda_{s}^{k \mathrm{~d}}-A^{k}(s, T)+\theta_{s}\left(\Sigma^{k}(s, T)\right)\right] d s+\bar{Z}_{0}^{k}(T) \\
& +\int_{0}^{t}\left(1-H_{s-}^{k}\right) Z_{s-}^{k}(T) \Sigma^{k}(s, T) \sqrt{c_{s}} d W_{s} \\
& +\int_{0}^{t} \int_{\mathbb{R}^{d}}\left(1-H_{s-}^{k}\right) Z_{s-}^{k}(T)\left(e^{\left\langle\Sigma^{k}(s, T), x\right\rangle}-1\right)\left(\mu^{L}-\nu\right)(d s, d x) \\
& +\int_{0}^{t} \hat{H}_{s-}^{k} d Z_{s}^{\mathrm{d}}(T)+\int_{0}^{t}\left(R_{s}^{k} Z_{s}^{\mathrm{d}}(T)-Z_{s}^{k}(T)\right) d H_{s}^{k} .
\end{aligned}
$$

Since condition 3.22 is assumed to be satisfied, we have

$$
\begin{aligned}
& \int_{0}^{t}\left(1-H_{s}^{k}\right) Z_{s-}^{k}(T)\left[\lambda_{s}^{k \mathrm{~d}}-A^{k}(s, T)+\theta_{s}\left(\Sigma^{k}(s, T)\right)\right] d s \\
= & \int_{0}^{t}\left(1-H_{s}^{k}\right)\left(Z_{s-}^{k}(T)-R_{s}^{k} Z_{s}^{\mathrm{d}}(T)\right) \gamma_{s}^{k} d s \\
= & \int_{0}^{t}\left(1-H_{s}^{k}\right)\left(Z_{s}^{k}(T)-R_{s}^{k} Z_{s}^{\mathrm{d}}(T)\right) \gamma_{s}^{k} d s
\end{aligned}
$$


and it follows that

$$
\begin{aligned}
\bar{Z}_{t}^{k}(T)= & \bar{Z}_{0}^{k}(T)+\int_{0}^{t}\left(1-H_{s-}^{k}\right) Z_{s-}^{k}(T) \Sigma^{k}(s, T) \sqrt{c_{s}} d W_{s} \\
& +\int_{0}^{t} \int_{\mathbb{R}^{d}}\left(1-H_{s-}^{k}\right) Z_{s-}^{k}(T)\left(e^{\left\langle\Sigma^{k}(s, T), x\right\rangle}-1\right)\left(\mu^{L}-\nu\right)(d s, d x) \\
& +\int_{0}^{t} \hat{H}_{s-}^{k} d Z_{s}^{\mathrm{d}}(T)+\int_{0}^{t}\left(R_{s}^{k} Z_{s}^{\mathrm{d}}(T)-Z_{s}^{k}(T)\right) d M_{s}^{k}
\end{aligned}
$$

where the $\left(\mathbb{G}, P^{\mathrm{d}}\right)$-martingale $M^{k}=\left(M_{t}^{k}\right)_{t \in\left[0, T^{*}\right]}$ is defined by

$$
M_{t}^{k}=H_{t}^{k}-\int_{0}^{t}\left(1-H_{s}^{k}\right) \gamma_{s}^{k} d s
$$

By taking into account the valid martingale invariance property, we observe that all the considered stochastic integrals in equation 3.23 have $\left(\mathbb{G}, P^{\mathrm{d}}\right)$-local martingales as integrators. Hence, we conclude that $\bar{Z}^{k}(T)$ is a $\left(\mathbb{G}, P^{\mathrm{d}}\right)$-local martingale.

Let us assume that the terminal recovery process is of the form

$$
R_{t}^{k}=R^{k} B_{t-}^{k}(T) B_{t}^{\mathrm{d}}(T)^{-1}, \text { where } R^{k} \in[0,1) .
$$

Note that this choice corresponds to the fractional recovery of market value (see Bielecki and Rutkowski [12, section 1.1.1]). By easy computations, we obtain the following practical form of condition (3.22): For each $k \in\{1, \ldots, m\}$ and $T \in\left[0, T^{*}\right]$, it holds

$$
\lambda_{t}^{k, \mathrm{~d}}-A^{k}(t, T)+\theta_{t}\left(\Sigma^{k}(t, T)\right)=\left(1-R^{k}\right) \gamma_{t}^{k}
$$

for every $t \in[0, T]$ (cf. Crépey, Grbac, and Nguyen [23, equation (26)]). One verifies that condition (3.24) can equivalently be formulated as

$$
\begin{aligned}
\lambda_{t}^{k, \mathrm{~d}} & =\left(1-R^{k}\right) \gamma_{t}^{k} \\
A^{k}(t, T) & =\theta_{t}\left(\Sigma^{k}(t, T)\right) .
\end{aligned}
$$

Hence, the credit risk component of the model is given by equation 3.25 .

Since the crisis was caused by a mixture of credit and liquidity risk (cf. Filipović and Trolle [45] and Eberlein [30]), we add a further liquidity component to the pure credit risk factor $\theta_{t}\left(\Sigma^{k}(t, T)\right)$ in 3.25 . Thus, we need another ingredient in the model:

$(\mathbb{M C} .3)$ We consider the liquidity component $l^{k}$ defined by

$$
l^{k}:\left\{\begin{array}{l}
{\left[0, T^{*}\right] \times\left[0, T^{*}\right] \rightarrow \mathbb{R}} \\
(t, T) \mapsto l^{k}(t, T)
\end{array}\right.
$$


which is assumed to be a deterministic, differentiable and bounded function.

Finally, we consider the drift function

$$
A^{k}(t, T)=\theta_{t}\left(\Sigma^{k}(t, T)\right)+l^{k}(t, T)
$$

for every $k \in\{1, \ldots, m\}$. We stress that the drift function $A^{k}$ (and $\alpha^{k}$ ) and the volatility function $\Sigma^{k}$ (and $\sigma^{k}$ ) are deterministic functions. Therefore, the factor $D^{k}$ is also deterministic. Observe also that this choice of the drift function corresponds to the fact that the forward reference rate $L^{k}(S, T)$ is not a $P_{T}^{\mathrm{d}}$-martingale in general.

\subsection{Monotonicity of the Curves}

Assumption (3.14) implies some kind of monotonicity of the curves. More specifically, bonds that are related to a riskier curve should have a lower price than bonds that correspond to a curve associated with less credit and liquidity risk. Figure 2.12 implicitly confirms this fact by means of market data.

\subsubsection{The Monotonicity Condition}

Let $m \in \mathbb{N}^{*}$. Recall that, by using the bootstrapping method of Ametrano and Bianchetti [2, 3], we generally have for every $k, l \in\{1, \ldots, m\}$ with $k \leq l$ and all $T \in\left[0, T^{*}\right]$ the monotonicity of the initial curves:

$$
B_{0}^{l}(T) \leq B_{0}^{k}(T) \leq B_{0}^{\mathrm{d}}(T)
$$

We have to design the model such that the monotonicity is valid for all dates. This means that we achieve the model quantities in such a way that we obtain

$$
B_{t}^{l}(T) \leq B_{t}^{k}(T) \leq B_{t}^{\mathrm{d}}(T)
$$

for every $t, T \in\left[0, T^{*}\right]$ satisfying $t \leq T$. This reflects the fact that the higher the risk is, the lower the price of the bond is. The monotonicity will be guaranteed by additional restrictions on the model parameters. The inequalities (3.27) obviously imply

$$
f_{t}^{\mathrm{d}}(T) \leq f_{t}^{k}(T) \leq f_{t}^{l}(T)
$$

by the relations 3.2 and 3.16 .

For every $k, j \in\{\mathrm{d}, 1 \ldots, m\}$ and $T \in\left[0, T^{*}\right]$, we define the additive (forward) spread between the curves $k$ and $j$ by

$$
s_{t}^{k, j}(T):=f_{t}^{k}(T)-f_{t}^{j}(T) .
$$


One sees that the dynamics $s^{k, j}(T)=\left(s_{t}^{k, j}(T)\right)_{t \in[0, T]}$ are given by

$$
s_{t}^{k, j}(T)=s_{0}^{k, j}(T)+\int_{0}^{t} \alpha^{k, j}(s, T) d s-\int_{0}^{t} \sigma^{k, j}(s, T) d L_{s},
$$

where we set $\alpha^{k, j}(s, T):=\alpha^{k}(s, T)-\alpha^{j}(s, T)$ and $\sigma^{k, j}(s, T):=\sigma^{k}(s, T)-\sigma^{j}(s, T)$. Note that $s_{t}^{k, k}(T)=0$ for every $k \in\{\mathrm{d}, 1, \ldots, m\}$ and we deduce from 3.28 that

$$
0 \leq s_{0}^{k, \mathrm{~d}}(T) \leq s_{0}^{l, \mathrm{~d}}(T) \quad \text { and } \quad 0 \leq s_{0}^{l, k}(T)
$$

for all $k, l \in\{1, \ldots, m\}$ with $k \leq l$ and $T \in\left[0, T^{*}\right]$. For every $k, j \in\{\mathrm{d}, 1 \ldots, m\}$, the short term spread between $k$ and $j$ is defined by $\lambda_{t}^{k, j}:=r_{t}^{k}-r_{t}^{j}$ and we set

$$
A^{k, j}(s, T):=\int_{s \wedge T}^{T} \alpha^{k, j}(s, u) d u \quad \text { and } \quad \Sigma^{k, j}(s, T):=\int_{s \wedge T}^{T} \sigma^{k, j}(s, u) d u .
$$

Then, we clearly have the relations

$$
A^{k, j}(s, T)=A^{k}(s, T)-A^{j}(s, T) \quad \text { and } \quad \Sigma^{k, j}(s, T)=\Sigma^{k}(s, T)-\Sigma^{j}(s, T) .
$$

For any $k \in\{1, \ldots, m\}$, we obviously have

$$
s_{t}^{k, \mathrm{~d}}(T)=\sum_{j=1}^{k} s_{t}^{j, j-1}(T),
$$

where we define $s_{t}^{1,0}(T):=s_{t}^{1, \mathrm{~d}}(T)$. Therefore, we obtain

$$
f_{t}^{k}(T)=f_{t}^{\mathrm{d}}(T)+\sum_{j=1}^{k} s_{t}^{j, j-1}(T) .
$$

Note that, for every $k \in\{1, \ldots, m\}$, the short term spread $\lambda^{k, \mathrm{~d}}$ is given by

$$
\lambda_{t}^{k, \mathrm{~d}}=\sum_{j=1}^{k} \lambda_{t}^{j, j-1}=\sum_{j=1}^{k} s_{t}^{j, j-1}(t),
$$

where we set $\lambda_{t}^{1,0}:=\lambda_{t}^{1, \mathrm{~d}}$. Moreover, one easily sees that the drift function and the volatility structure related to curve $k$ can be represented by

$$
\alpha^{k}(s, T)=\alpha^{\mathrm{d}}(s, T)+\sum_{j=1}^{k} \alpha^{j, j-1}(s, T)
$$


and

$$
\sigma^{k}(s, T)=\sigma^{\mathrm{d}}(s, T)+\sum_{j=1}^{k} \sigma^{j, j-1}(s, T),
$$

where we denote $\alpha^{1,0}(s, T):=\alpha^{1, \mathrm{~d}}(s, T)$ and $\sigma^{1,0}(s, T):=\sigma^{1, \mathrm{~d}}(s, T)$. It follows that we have

$$
A^{k}(s, T)=A^{\mathrm{d}}(s, T)+\sum_{j=1}^{k} A^{j, j-1}(s, T)
$$

and

$$
\Sigma^{k}(s, T)=\Sigma^{\mathrm{d}}(s, T)+\sum_{j=1}^{k} \Sigma^{j, j-1}(s, T),
$$

where we define $A^{1,0}(s, T):=A^{1, \mathrm{~d}}(s, T)$ and $\Sigma^{1,0}(s, T):=\Sigma^{1, \mathrm{~d}}(s, T)$. Consequently, due to relation 3.29 , we can specify the dynamics of the quantity $f^{k}(T)$ by modelling the forward spreads $s^{k, j}(T)$ and the forward rates $f^{j}(T)$.

It is evident that the relation 3.28 is equivalent to the condition

$$
0 \leq s_{t}^{k, \mathrm{~d}}(T) \leq s_{t}^{l, \mathrm{~d}}(T)
$$

for every $k, l \in\{1, \ldots, m\}$ with $k \leq l$ and $t, T \in\left[0, T^{*}\right]$ satisfying $t \leq T$. Then, we conclude from representation (3.31) that condition 3.32 is valid if, for all $j \in\{1, \ldots, m\}$ and $t, T \in\left[0, T^{*}\right]$ with $t \leq T$, we have

$$
0 \leq s_{t}^{j, j-1}(T)
$$

To sum up, this approach results in the non-negative specification of the forward spreads between two subsequent curves $(3.33)$. Then, condition $(3.32)$ is automatically satisfied. This fact implicitly guarantees the relations $(3.28)$ and we therefore ensure the required monotonicity (3.27).

We end this subsection with the derivation of a representation of the (forward) spreads.

Lemma 3.2.1 The forward spread $s_{t}^{1, \mathrm{~d}}(T)$ is given by

$$
\begin{aligned}
s_{t}^{1, \mathrm{~d}}(T)= & s_{0}^{1, \mathrm{~d}}(T)+\int_{0}^{t}\left[\frac{\partial}{\partial T} \theta_{s}\left(\Sigma^{\mathrm{d}}(s, T)+\Sigma^{1, \mathrm{~d}}(s, T)\right)+\frac{\partial}{\partial T} l^{1}(s, T)\right. \\
& \left.-\frac{\partial}{\partial T} \theta_{s}\left(\Sigma^{\mathrm{d}}(s, T)\right)\right] d s-\int_{0}^{t} \sigma^{1, \mathrm{~d}}(s, T) d L_{s}
\end{aligned}
$$


and, in the case of $m \geq 2$ with $j \in\{2, \ldots, m\}$, the forward spread $s_{t}^{j, j-1}(T)$ can be represented by

$$
\begin{aligned}
s_{t}^{j, j-1}(T)= & s_{0}^{j, j-1}(T)+\int_{0}^{t}\left[\frac{\partial}{\partial T} \theta_{s}\left(\Sigma^{\mathrm{d}}(s, T)+\sum_{i=1}^{j} \Sigma^{i, i-1}(s, T)\right)+\frac{\partial}{\partial T} l^{j, j-1}(s, T)\right. \\
& \left.-\frac{\partial}{\partial T} \theta_{s}\left(\Sigma^{\mathrm{d}}(s, T)+\sum_{i=1}^{j-1} \Sigma^{i, i-1}(s, T)\right)\right] d s-\int_{0}^{t} \sigma^{j, j-1}(s, T) d L_{s},
\end{aligned}
$$

where we set $l^{j, j-1}(s, T):=l^{j}(s, T)-l^{j-1}(s, T)$.

Proof: On the basis of the specifications of the drift functions 3.7$)$ and 3.26 , we get

$$
\alpha^{1,0}(s, T)=\alpha^{1, \mathrm{~d}}(s, T)=\frac{\partial}{\partial T} \theta_{s}\left(\Sigma^{1}(s, T)\right)+\frac{\partial}{\partial T} l^{1}(s, T)-\frac{\partial}{\partial T} \theta_{s}\left(\Sigma^{\mathrm{d}}(s, T)\right)
$$

and, for $m \geq 2$ where $j \in\{2, \ldots, m\}$, it holds

$$
\alpha^{j, j-1}(s, T)=\frac{\partial}{\partial T} \theta_{s}\left(\Sigma^{j}(s, T)\right)+\frac{\partial}{\partial T} l^{j}(s, T)-\frac{\partial}{\partial T} \theta_{s}\left(\Sigma^{j-1}(s, T)\right)-\frac{\partial}{\partial T} l^{j-1}(s, T) .
$$

Then, the assertion follows from the forward spreads dynamics given by 3.30 .

\subsubsection{A Suitable Model Framework}

We present an example of a tractable model which ensures the non-negativity of the consecutive forward spreads. Through the analysis made in the previous subsection, it follows that the monotonicity of the curves (3.27) is then valid. This framework is motivated by Crépey, Grbac, and Nguyen [23]. We emphasise that we specify the model quantities corresponding to each curve $k \in\{1, \ldots, m\}$ by modelling the quantities of the relevant spreads and the discount curve (cf. the previous subsection and relation (3.29)).

Let $d, m, l \in \mathbb{N}^{*}=\{1,2,3, \ldots\}$ with $l+m \leq d$. The $d$-dimensional driving process $L=\left(L^{1}, \ldots, L^{d}\right)^{\top}$ is given on the enlarged stochastic basis $\left(\Omega, \mathscr{G}, \mathbb{G}, P^{\mathrm{d}}\right)$. Its components are divided into $l$ real-valued Lévy processes and $d-l$ negative Lévy processes. More precisely, we specify the $d$-dimensional Lévy process $L$ as follows:

(i) $Y^{1}:=\left(L^{1}, \ldots, L^{l}\right)^{\top}$ is an $\mathbb{R}^{l}$-valued Lévy process.

(ii) $Y^{2}:=\left(L^{l+1}, \ldots, L^{d}\right)^{\top}=\left(-Z^{l+1}, \ldots,-Z^{d}\right)^{\top}$, where $Z:=\left(Z^{l+1}, \ldots, Z^{d}\right)^{\top}=-Y^{2}$ is an $\mathbb{R}_{+}^{d-l}$-valued Lévy process whose components are subordinators (see Sato [81, Definition 21.4.] and Barndorff-Nielsen and Shephard [8]). The cumulant process of $Z$ is of the form

$$
\theta^{Z}(z)=\langle z, b\rangle+\int_{\mathbb{R}_{+}^{d-l}}\left(e^{\langle z, x\rangle}-1\right) F(d x),
$$


where $z \in \mathbb{C}^{d-l}$ such that $\operatorname{Re}(z) \in[-(1+\epsilon) M,(1+\epsilon) M]^{d-l}$. The drift term $b$ satisfies $b^{j} \geq 0$ for any $j \in\{1, \ldots, d-l\}$ and the Lévy measure $F$ has its support on $\mathbb{R}_{+}^{d-l}$.

We make the following standing assumption:

Assumption $(\mathbb{V L}):$ For every $k \in\{1, \ldots, m\}$, the non-negative volatility functions $\Sigma^{\mathrm{d}}$ and $\Sigma^{k, k-1}$ as well as the liquidity function $l^{k}$ are deterministic, differentiable and stationary functions. This means that for every $k \in\{1, \ldots, m\}, j \in\{1, \ldots, d\}$ and $s, T$ with $0 \leq s \leq T \leq T^{*}$, the functions are of the form

$$
\begin{aligned}
\Sigma_{j}^{\mathrm{d}}(s, T) & =G_{j}(T-s) \\
\Sigma_{j}^{k, k-1}(s, T) & =G_{j}^{k}(T-s) \\
l^{k}(s, T) & =G_{l}^{k}(T-s),
\end{aligned}
$$

where $G_{j}:\left[0, T^{*}\right] \rightarrow \mathbb{R}_{+}$and $G_{j}^{k}:\left[0, T^{*}\right] \rightarrow \mathbb{R}_{+}$are differentiable and deterministic functions satisfying $G_{j}(0)=G_{j}^{k}(0)=0$ that are bounded in the sense of

$$
G_{j}(s)+\sum_{k=1}^{m} G_{j}^{k}(s) \leq \hat{M}<M
$$

for all $s \in\left[0, T^{*}\right]$ with the constant $M$ from assumption $(\mathbb{E M})$, and $G_{l}^{k}:\left[0, T^{*}\right] \rightarrow \mathbb{R}$ is a differentiable, deterministic and bounded function satisfying $G_{l}^{k}(0)=0$.

It follows that the conditions $(\mathbb{D E T})$ and $(\mathbb{M C} . \mathbb{D E T})$ are fulfilled under assumption $(\mathbb{V} \mathbb{L})$.

Proposition 3.2.1 The forward spread $s_{t}^{1, \mathrm{~d}}(T)$ can be written as

$$
\begin{aligned}
s_{t}^{1, \mathrm{~d}}(T)= & s_{0}^{1, \mathrm{~d}}(T)-\theta\left(\Sigma^{\mathrm{d}}(t, T)+\Sigma^{1, \mathrm{~d}}(t, T)\right)+\theta\left(\Sigma^{\mathrm{d}}(0, T)+\Sigma^{1, \mathrm{~d}}(0, T)\right) \\
& -l^{1}(t, T)+l^{1}(0, T)+\theta\left(\Sigma^{\mathrm{d}}(t, T)\right)-\theta\left(\Sigma^{\mathrm{d}}(0, T)\right) \\
& -\int_{0}^{t} \sigma^{1, \mathrm{~d}}(s, T) d L_{s}
\end{aligned}
$$


and, in the case where $m \geq 2$, the forward spread $s_{t}^{j, j-1}(T)$ is given by

$$
\begin{aligned}
s_{t}^{j, j-1}(T)= & s_{0}^{j, j-1}(T)-\theta\left(\Sigma^{\mathrm{d}}(t, T)+\sum_{i=1}^{j} \Sigma^{i, i-1}(t, T)\right)+\theta\left(\Sigma^{\mathrm{d}}(0, T)+\sum_{i=1}^{j} \Sigma^{i, i-1}(0, T)\right) \\
& -l^{j, j-1}(t, T)+l^{j, j-1}(0, T)+\theta\left(\Sigma^{\mathrm{d}}(t, T)+\sum_{i=1}^{j-1} \Sigma^{i, i-1}(t, T)\right) \\
& -\theta\left(\Sigma^{\mathrm{d}}(0, T)+\sum_{i=1}^{j-1} \Sigma^{i, i-1}(0, T)\right)-\int_{0}^{t} \sigma^{j, j-1}(s, T) d L_{s}
\end{aligned}
$$

for any $j \in\{2, \ldots, m\}$. The corresponding short spreads result in

$$
\lambda_{t}^{1, \mathrm{~d}}=s_{0}^{1, \mathrm{~d}}(t)+\theta\left(\Sigma^{\mathrm{d}}(0, t)+\Sigma^{1, \mathrm{~d}}(0, t)\right)+l^{1}(0, t)-\theta\left(\Sigma^{\mathrm{d}}(0, t)\right)-\int_{0}^{t} \sigma^{1, \mathrm{~d}}(s, t) d L_{s}
$$

and

$$
\begin{aligned}
\lambda_{t}^{j, j-1}= & s_{0}^{j, j-1}(t)+\theta\left(\Sigma^{\mathrm{d}}(0, t)+\sum_{i=1}^{j} \Sigma^{i, i-1}(0, t)\right)+l^{j, j-1}(0, t) \\
& -\theta\left(\Sigma^{\mathrm{d}}(0, t)+\sum_{i=1}^{j-1} \Sigma^{i, i-1}(0, t)\right)-\int_{0}^{t} \sigma^{j, j-1}(s, t) d L_{s} .
\end{aligned}
$$

Proof: We first observe that it holds

$$
\begin{aligned}
\frac{\partial}{\partial T} G_{j}(T-s) & =-\frac{\partial}{\partial s} G_{j}(T-s) \\
\frac{\partial}{\partial T} G_{j}^{k}(T-s) & =-\frac{\partial}{\partial s} G_{j}^{k}(T-s) \\
\frac{\partial}{\partial T} G_{l}^{k}(T-s) & =-\frac{\partial}{\partial s} G_{l}^{k}(T-s) .
\end{aligned}
$$

Then, let us consider the representation of $s_{t}^{1, \mathrm{~d}}(T)$ and $s_{t}^{j, j-1}(T)$ that are stated in Lemma 3.2.1. It immediately follows that

$$
\begin{aligned}
& \frac{\partial}{\partial T} \theta\left(\Sigma^{\mathrm{d}}(s, T)+\Sigma^{1, \mathrm{~d}}(s, T)\right)+\frac{\partial}{\partial T} l^{1}(s, T)-\frac{\partial}{\partial T} \theta\left(\Sigma^{\mathrm{d}}(s, T)\right) \\
= & -\frac{\partial}{\partial s} \theta\left(\Sigma^{\mathrm{d}}(s, T)+\Sigma^{1, \mathrm{~d}}(s, T)\right)-\frac{\partial}{\partial s} l^{1}(s, T)+\frac{\partial}{\partial s} \theta\left(\Sigma^{\mathrm{d}}(s, T)\right)
\end{aligned}
$$


from which we observe that

$$
\begin{aligned}
& \int_{0}^{t}\left[\frac{\partial}{\partial T} \theta\left(\Sigma^{\mathrm{d}}(s, T)+\Sigma^{1, \mathrm{~d}}(s, T)\right)+\frac{\partial}{\partial T} l^{1}(s, T)-\frac{\partial}{\partial T} \theta\left(\Sigma^{\mathrm{d}}(s, T)\right)\right] d s \\
= & -\int_{0}^{t}\left[\frac{\partial}{\partial s} \theta\left(\Sigma^{\mathrm{d}}(s, T)+\Sigma^{\mathrm{d}}(s, T)\right)+\frac{\partial}{\partial s} l^{1}(s, T)-\frac{\partial}{\partial s} \theta\left(\Sigma^{\mathrm{d}}(s, T)\right)\right] d s \\
= & -\theta\left(\Sigma^{\mathrm{d}}(t, T)+\Sigma^{1, \mathrm{~d}}(t, T)\right)+\theta\left(\Sigma^{\mathrm{d}}(0, T)+\Sigma^{1, \mathrm{~d}}(0, T)\right)-l^{1}(t, T)+l^{1}(0, T) \\
& +\theta\left(\Sigma^{\mathrm{d}}(t, T)\right)-\theta\left(\Sigma^{\mathrm{d}}(0, T)\right) .
\end{aligned}
$$

Hence, $s_{t}^{1, \mathrm{~d}}(T)$ can be expressed in the form 3.34. Analogously, one shows that

$$
\begin{aligned}
& \frac{\partial}{\partial T} \theta\left(\Sigma^{\mathrm{d}}(s, T)+\sum_{i=1}^{j} \Sigma^{i, i-1}(s, T)\right)+\frac{\partial}{\partial T} l^{j, j-1}(s, T) \\
& -\frac{\partial}{\partial T} \theta\left(\Sigma^{\mathrm{d}}(s, T)+\sum_{i=1}^{j-1} \Sigma^{i, i-1}(s, T)\right) \\
= & -\frac{\partial}{\partial s} \theta\left(\Sigma^{\mathrm{d}}(s, T)+\sum_{i=1}^{j} \Sigma^{i, i-1}(s, T)\right)-\frac{\partial}{\partial s} l^{j, j-1}(s, T) \\
& +\frac{\partial}{\partial s} \theta\left(\Sigma^{\mathrm{d}}(s, T)+\sum_{i=1}^{j-1} \Sigma^{i, i-1}(s, T)\right) .
\end{aligned}
$$

Then, we get

$$
\begin{aligned}
& \int_{0}^{t}\left[\frac{\partial}{\partial T} \theta\left(\Sigma^{\mathrm{d}}(s, T)+\sum_{i=1}^{j} \Sigma^{i, i-1}(s, T)\right)+\frac{\partial}{\partial T} l^{j, j-1}(s, T)\right. \\
& \left.-\frac{\partial}{\partial T} \theta\left(\Sigma^{\mathrm{d}}(s, T)+\sum_{i=1}^{j-1} \Sigma^{i, i-1}(s, T)\right)\right] d s \\
= & -\int_{0}^{t}\left[\frac{\partial}{\partial s} \theta\left(\Sigma^{\mathrm{d}}(s, T)+\sum_{i=1}^{j} \Sigma^{i, i-1}(s, T)\right)+\frac{\partial}{\partial s} l^{j, j-1}(s, T)\right. \\
& \left.-\frac{\partial}{\partial s} \theta\left(\Sigma^{\mathrm{d}}(s, T)+\sum_{i=1}^{j-1} \Sigma^{i, i-1}(s, T)\right)\right] d s \\
= & -\theta\left(\Sigma^{\mathrm{d}}(t, T)+\sum_{i=1}^{j} \Sigma^{i, i-1}(t, T)\right)+\theta\left(\Sigma^{\mathrm{d}}(0, T)+\sum_{i=1}^{j} \Sigma^{i, i-1}(0, T)\right)-l^{j, j-1}(t, T) \\
& +l^{j, j-1}(0, T)+\theta\left(\Sigma^{\mathrm{d}}(t, T)+\sum_{i=1}^{j-1} \Sigma^{i, i-1}(t, T)\right)-\theta\left(\Sigma^{\mathrm{d}}(0, T)+\sum_{i=1}^{j-1} \Sigma^{i, i-1}(0, T)\right) .
\end{aligned}
$$


The last expressions immediately lead to the form 3.35. By definition, we easily obtain the representations of the short spreads.

Next, we derive necessary and sufficient deterministic conditions for the non-negativity of the (consecutive) forward spreads. We explicitly mention that this approach is closely related to the proceeding of Crépey, Grbac, and Nguyen [23, section 3.2].

Let $m \geq 2$ and define the deterministic terms

$$
\begin{aligned}
\mu^{1, \mathrm{~d}}(t, T):= & s_{t}^{1, \mathrm{~d}}(T)+\int_{0}^{t} \sigma^{1, \mathrm{~d}}(s, T) d L_{s} \\
= & s_{0}^{1, \mathrm{~d}}(T)-\theta\left(\Sigma^{\mathrm{d}}(t, T)+\Sigma^{1, \mathrm{~d}}(t, T)\right)+\theta\left(\Sigma^{\mathrm{d}}(0, T)+\Sigma^{1, \mathrm{~d}}(0, T)\right) \\
& -l^{1}(t, T)+l^{1}(0, T)+\theta\left(\Sigma^{\mathrm{d}}(t, T)\right)-\theta\left(\Sigma^{\mathrm{d}}(0, T)\right) \\
\mu^{1, \mathrm{~d}}(t):= & \lambda_{t}^{1, \mathrm{~d}}+\int_{0}^{t} \sigma^{1, \mathrm{~d}}(s, t) d L_{s} \\
= & s_{0}^{1, \mathrm{~d}}(t)+\theta\left(\Sigma^{\mathrm{d}}(0, t)+\Sigma^{1, \mathrm{~d}}(0, t)\right)+l^{1}(0, t)-\theta\left(\Sigma^{\mathrm{d}}(0, t)\right)
\end{aligned}
$$

and

$$
\begin{aligned}
\mu^{j, j-1}(t, T):= & s_{t}^{j, j-1}(T)+\int_{0}^{t} \sigma^{j, j-1}(s, T) d L_{s} \\
= & s_{0}^{j, j-1}(T)-\theta\left(\Sigma^{\mathrm{d}}(t, T)+\sum_{i=1}^{j} \Sigma^{i, i-1}(t, T)\right) \\
& +\theta\left(\Sigma^{\mathrm{d}}(0, T)+\sum_{i=1}^{j} \Sigma^{i, i-1}(0, T)\right)-l^{j, j-1}(t, T)+l^{j, j-1}(0, T) \\
& +\theta\left(\Sigma^{\mathrm{d}}(t, T)+\sum_{i=1}^{j-1} \Sigma^{i, i-1}(t, T)\right)-\theta\left(\Sigma^{\mathrm{d}}(0, T)+\sum_{i=1}^{j-1} \Sigma^{i, i-1}(0, T)\right) \\
\mu^{j, j-1}(t):= & \lambda_{t}^{j, j-1}+\int_{0}^{t} \sigma^{j, j-1}(s, t) d L_{s} \\
= & s_{0}^{j, j-1}(t)+\theta\left(\Sigma^{\mathrm{d}}(0, t)+\sum_{i=1}^{j} \Sigma^{i, i-1}(0, t)\right)+l^{j, j-1}(0, t) \\
& -\theta\left(\Sigma^{\mathrm{d}}(0, t)+\sum_{i=1}^{j-1} \Sigma^{i, i-1}(0, t)\right)
\end{aligned}
$$

respectively.

Proposition 3.2.2 Let $T \in\left[0, T^{*}\right]$. We assume that the following two conditions are satisfied: 
1. For any $t \in[0, T]$ and each $k \in\{1, \ldots, l\}$, we have

$$
\sigma_{k}^{1, \mathrm{~d}}(t, T)=0
$$

and, if $m \geq 2$, it holds

$$
\sigma_{k}^{j, j-1}(t, T)=0
$$

for every $j \in\{2, \ldots, m\}$.

2. For all $t \in[0, T]$, it holds

$$
0 \leq \mu^{1, \mathrm{~d}}(t, T)=\mu^{1, \mathrm{~d}}(T)-\theta\left(\Sigma^{\mathrm{d}}(t, T)+\Sigma^{1, \mathrm{~d}}(t, T)\right)-l^{1}(t, T)+\theta\left(\Sigma^{\mathrm{d}}(t, T)\right)
$$

and, if $m \geq 2$, we have

$$
\begin{aligned}
0 \leq & \mu^{j, j-1}(t, T)=\mu^{j, j-1}(T)-\theta\left(\Sigma^{\mathrm{d}}(t, T)+\sum_{i=1}^{j} \Sigma^{i, i-1}(t, T)\right)-l^{j, j-1}(t, T) \\
& +\theta\left(\Sigma^{\mathrm{d}}(t, T)+\sum_{i=1}^{j-1} \Sigma^{i, i-1}(t, T)\right)
\end{aligned}
$$

for every $j \in\{2, \ldots, m\}$.

Then, it results that the forward spreads meet

$$
0 \leq s_{t}^{1, \mathrm{~d}}(T) \quad \text { and } \quad 0 \leq s_{t}^{j, j-1}(T)
$$

for every $t, T \in\left[0, T^{*}\right]$ with $t \leq T$ and each $j \in\{2, \ldots, m\}$.

Proof: It follows from the definition of $\mu^{1, \mathrm{~d}}(t, T)$ that the non-negativity of the term $-\int_{0}^{t} \sigma^{1, \mathrm{~d}}(s, T) d L_{s}$, for any $t \in[0, T]$, together with 3.36 imply $s_{t}^{1, \mathrm{~d}}(T) \geq 0$ for all $t \in[0, T]$. Similar arguments lead to the non-negativity of the spread $s_{t}^{j, j-1}(T)$ for any $t \in[0, T]$ with $t \leq T$ and every $j \in\{2, \ldots, m\}$ if $m \geq 2$.

Note that the conditions (3.36) and (3.37) result in additional restrictions on the considered distribution parameters of the driving process as well as the parameters of the volatility and liquidity functions. 


\subsection{Valuation Formulas with Application of the Two-Price Theory}

In this section, we specify mid price valuation formulas of interest rate derivatives in the multiple-curve Lévy forward rate model. Furthermore, the two-price theory is applied to some special financial products and the corresponding ask and bid prices are derived.

\subsubsection{Valuation Formulas of Interest Rate Derivatives}

We begin with some preliminary remarks related to the valuation approach. Let $T^{*}>0$ be a finite time horizon and $m \in \mathbb{N}$. Recall that in the case where $m=0$, we obtain the classical single-curve setting and the discount curve is referred to as 0 or d. For any $k \in\{0,1, \ldots, m\}$, let $\mathscr{T}^{k}=\left\{T_{0}^{k}, \ldots, T_{n_{k}}^{k}\right\}$ be an equidistant discrete tenor structure with tenor $\delta^{k}$. We assume that $0 \leq T_{0}^{k}=T_{0}^{l}, T_{n_{k}}^{k}=T^{*}$ and $\mathscr{T}^{l} \subset \mathscr{T}^{k}$ for every $k, l \in\{0,1, \ldots, m\}$ satisfying $k \leq l\left(\delta^{k}<\delta^{l}\right)$. To simplify the notation, we omit the superscripts for the dates and tenors related to curve 0 (or $\mathrm{d}$, respectively).

We conclude from the equations 3.11 and 3.19 that the time- $T_{j-1}^{k}$ bond price maturing at date $T_{j}^{k}$ can be represented by

$$
\begin{aligned}
B_{T_{j-1}}^{\mathrm{d}}\left(T_{j}\right)=\frac{B_{0}^{\mathrm{d}}\left(T_{j}\right)}{B_{0}^{\mathrm{d}}\left(T_{j-1}\right)} \exp ( & \int_{0}^{T_{j-1}} \Sigma^{\mathrm{d}}\left(s, T_{j-1}, T_{j}\right) d L_{s} \\
& \left.+\int_{0}^{T_{j-1}}\left[\theta_{s}\left(\Sigma^{\mathrm{d}}\left(s, T_{j-1}\right)\right)-\theta_{s}\left(\Sigma^{\mathrm{d}}\left(s, T_{j}\right)\right)\right] d s\right)
\end{aligned}
$$

for every $j \in\{1, \ldots, n\}$ and

$$
\begin{aligned}
B_{T_{j-1}^{k}}^{k}\left(T_{j}^{k}\right)=\frac{B_{0}\left(T_{j}^{k}\right)}{B_{0}\left(T_{j-1}^{k}\right)} \exp ( & \int_{0}^{T_{j-1}^{k}} \Sigma^{k}\left(s, T_{j-1}^{k}, T_{j}^{k}\right) d L_{s} \\
& +\int_{0}^{T_{j-1}^{k}}\left[\theta_{s}\left(\Sigma^{k}\left(s, T_{j-1}^{k}\right)\right)+l^{k}\left(s, T_{j-1}^{k}\right)\right. \\
& \left.\left.-\theta_{s}\left(\Sigma^{k}\left(s, T_{j}^{k}\right)\right)-l^{k}\left(s, T_{j}^{k}\right)\right] d s\right)
\end{aligned}
$$

for every $j \in\left\{1, \ldots, n_{k}\right\}$ with $k \in\{1, \ldots, m\}$. Recall that these expressions can compactly be written as

$$
B_{T_{j-1}^{k}}^{k}\left(T_{j}^{k}\right)=D_{j}^{k} \exp \left(X_{j}^{k}\right)
$$


where we set the deterministic part as

$$
D_{j}^{k}:=D^{k}\left(T_{j-1}^{k}, T_{j}^{k}\right)
$$

and the stochastic term as

$$
X_{j}^{k}:=X^{k}\left(T_{j-1}^{k}, T_{j}^{k}\right)
$$

for every $k \in\{\mathrm{d}, 1, \ldots, m\}$ (cf. equations 3.12, 3.13, 3.20) and (3.21)). Furthermore, the discount factor process $\beta^{\text {d }}$ at date $T \in\left[0, T^{*}\right]$ is calculated as

$$
\beta_{T}^{\mathrm{d}}=B_{0}^{\mathrm{d}}(T) \exp \left(-\int_{0}^{T} \theta_{s}\left(\Sigma^{\mathrm{d}}(s, T)\right) d s+\int_{0}^{T} \Sigma^{\mathrm{d}}(s, T) d L_{s}\right) .
$$

By using Eberlein and Raible [38, Lemma 3.1] or its generalised version developed by Eberlein and Kluge [33], we observe that the characteristic function of $X_{j}^{k}$ under $P_{T}^{\mathrm{d}}$ can be determined as

$$
\begin{aligned}
\varphi_{X_{j}^{k}}^{T}(u) & =\mathbb{E}_{P_{T}^{\mathrm{d}}}\left[\exp \left(\int_{0}^{T_{j-1}^{k}} i u \Sigma^{k}\left(s, T_{j-1}^{k}, T_{j}^{k}\right) d L_{s}\right)\right] \\
& =\exp \left(\int_{0}^{T_{j-1}^{k}} \theta_{s}^{T}\left(i u \Sigma^{k}\left(s, T_{j-1}^{k}, T_{j}^{k}\right)\right) d s\right),
\end{aligned}
$$

where $\theta_{s}^{T}$ denotes the cumulant with respect to $P_{T}^{\mathrm{d}}$ and $T \in \mathscr{T}^{\mathrm{d}}$. By changing to the spot martingale measure $P^{\mathrm{d}}$ with the use of 2.13 together with equation 3.5 (concretely, (3.38), we get the useful representations

$$
\begin{aligned}
\varphi_{X_{j}^{k}}^{T_{j}^{k}}(u) & =\mathbb{E}_{P^{\mathrm{d}}}\left[\beta_{T_{j}^{k}}^{\mathrm{d}} B_{0}^{\mathrm{d}}\left(T_{j}^{k}\right)^{-1} \exp \left(\int_{0}^{T_{j-1}^{k}} i u \Sigma^{k}\left(s, T_{j-1}^{k}, T_{j}^{k}\right) d L_{s}\right)\right] \\
& =\exp \left(\int_{0}^{T_{j-1}^{k}}\left[\theta_{s}\left(\Sigma^{\mathrm{d}}\left(s, T_{j}^{k}\right)+i u \Sigma^{k}\left(s, T_{j-1}^{k}, T_{j}^{k}\right)\right)-\theta_{s}\left(\Sigma^{\mathrm{d}}\left(s, T_{j}^{k}\right)\right)\right] d s\right)
\end{aligned}
$$

and

$$
\begin{aligned}
\varphi_{X_{j}^{\mathrm{d}}}^{T_{j-1}}(u) & =\mathbb{E}_{P^{\mathrm{d}}}\left[\beta_{T_{j-1}}^{\mathrm{d}} B_{0}^{\mathrm{d}}\left(T_{j-1}\right)^{-1} \exp \left(\int_{0}^{T_{j-1}} i u \Sigma^{\mathrm{d}}\left(s, T_{j-1}, T_{j}\right) d L_{s}\right)\right] \\
& =\exp \left(\int_{0}^{T_{j-1}}\left[\theta_{s}\left(\Sigma^{\mathrm{d}}\left(s, T_{j-1}\right)+i u \Sigma^{\mathrm{d}}\left(s, T_{j-1}, T_{j}\right)\right)-\theta_{s}\left(\Sigma^{\mathrm{d}}\left(s, T_{j-1}\right)\right)\right] d s\right) .
\end{aligned}
$$


Note that these expressions can be extended to complex numbers by using assumption (EM) (cf. Sato [81, Theorem 25.17]).

The payoffs of the derivatives that we will consider are combinations of deterministic functions of the reference rates $L_{T_{j-1}^{k}}^{k}\left(T_{j-1}^{k}, T_{j}^{k}\right)$. These rates are $\mathscr{F}_{T_{-1}^{k}}$-measurable random variables. Moreover, the discount factor process $\beta^{\mathrm{d}}$ is $\mathbb{F}$-adapted. Since the martingale invariance property between $\mathbb{F}$ and $\mathbb{G}$ under $P^{\mathrm{d}}$ is valid, we conclude from hypothesis $(\mathcal{H} 5)$ of subsection 1.2 .1 that it holds

$$
\left(\beta_{t}^{\mathrm{d}}\right)^{-1} \mathbb{E}_{P^{\mathrm{d}}}\left[\beta_{T_{j}^{k}}^{\mathrm{d}} f\left(L_{T_{j-1}^{k}}^{k}\left(T_{j-1}^{k}, T_{j}^{k}\right)\right) \mid \mathscr{G}_{t}\right]=\left(\beta_{t}^{\mathrm{d}}\right)^{-1} \mathbb{E}_{P^{\mathrm{d}}}\left[\beta_{T_{j}^{k}}^{\mathrm{d}} f\left(L_{T_{j-1}^{k}}^{k}\left(T_{j-1}^{k}, T_{j}^{k}\right)\right) \mid \mathscr{F}_{t}\right]
$$

for every $t \in\left[0, T_{j-1}^{k}\right]$ and each Borel-measurable deterministic function $f: \mathbb{R} \rightarrow \mathbb{R}$. Note that $f\left(L_{T_{j-1}^{k}}^{k}\left(T_{j-1}^{k}, T_{j}^{k}\right)\right)$ is considered as an integrable $\mathscr{F}_{T_{j-1}^{k}}^{k}$-measurable random variable. By using the abstract Bayes rule, we obtain

$$
\left(\beta_{t}^{\mathrm{d}}\right)^{-1} \mathbb{E}_{P^{\mathrm{d}}}\left[\beta_{T_{j}^{k}}^{\mathrm{d}} f\left(L_{T_{j-1}^{k}}^{k}\left(T_{j-1}^{k}, T_{j}^{k}\right)\right) \mid \mathscr{G}_{t}\right]=\mathbb{E}_{P_{T_{j}^{k}}^{\mathrm{d}}}\left[B_{t}^{\mathrm{d}}\left(T_{j}^{k}\right) f\left(L_{T_{j-1}^{k}}^{k}\left(T_{j-1}^{k}, T_{j}^{k}\right)\right) \mid \mathscr{G}_{t}\right] .
$$

From the notes made in subsection 1.2.1, we conclude that the martingale invariance property between $\mathbb{F}$ and $\mathbb{G}$ is also satisfied for any forward measure $P_{T}^{\mathrm{d}}$ with $T \in\left[0, T^{*}\right]$. Hence, we have

$$
\mathbb{E}_{P_{T_{j}^{k}}^{\mathrm{d}}}\left[B_{t}^{\mathrm{d}}\left(T_{j}^{k}\right) f\left(L_{T_{j-1}^{k}}^{k}\left(T_{j-1}^{k}, T_{j}^{k}\right)\right) \mid \mathscr{G}_{t}\right]=\mathbb{E}_{P_{T_{j}^{k}}^{\mathrm{d}}}\left[B_{t}^{\mathrm{d}}\left(T_{j}^{k}\right) f\left(L_{T_{j-1}^{k}}^{k}\left(T_{j-1}^{k}, T_{j}^{k}\right)\right) \mid \mathscr{F}_{t}\right]
$$

for every $t \in\left[0, T_{j-1}^{k}\right]$. To summarise, we can replace $\mathscr{G}_{t}$ by $\mathscr{F}_{t}$ in all risk-neutral pricing formulas in our model.

Now, we consider $k \in\{\mathrm{d}, 1, \ldots, m\}$. The related valuation formulas for the single-curve setting are well-known and can be found in the literature. To simplify the notation, the notional amount is set to one.

\section{Forward Rate Agreements}

Recall that the payoff of a forward rate agreement at maturity $T_{j}^{k}$ is given by

$$
\operatorname{FRA}_{T_{j}^{k}}\left(T_{j-1}^{k}, T_{j}^{k}, \delta^{k}, K\right)=\delta^{k}\left(L_{T_{j-1}^{k}}^{k}\left(T_{j-1}^{k}, T_{j}^{k}\right)-K\right) .
$$


Then, the value of this product at time $t \leq T_{j-1}^{k}$ is

$$
\begin{aligned}
\operatorname{FRA}_{t}\left(T_{j-1}^{k}, T_{j}^{k}, \delta^{k}, K\right) & =\delta^{k}\left(\beta_{t}^{\mathrm{d}}\right)^{-1} \mathbb{E}_{P^{\mathrm{d}}}\left[\beta_{T_{j}^{k}}^{\mathrm{d}}\left(L_{T_{j-1}^{k}}^{k}\left(T_{j-1}^{k}, T_{j}^{k}\right)-K\right) \mid \mathscr{F}_{t}\right] \\
& =\left(\beta_{t}^{\mathrm{d}}\right)^{-1} \mathbb{E}_{P^{\mathrm{d}}}\left[\beta_{T_{j}^{k}}^{\mathrm{d}}\left(B_{T_{j-1}^{k}}^{k}\left(T_{j}^{k}\right)^{-1}-\tilde{K}^{k}\right) \mid \mathscr{F}_{t}\right] \\
& =B_{t}^{\mathrm{d}}\left(T_{j}^{k}\right) \mathbb{E}_{P_{T_{j}^{k}}^{\mathrm{d}}}\left[\left(B_{T_{j-1}^{k}}^{k}\left(T_{j}^{k}\right)^{-1}-\tilde{K}^{k}\right) \mid \mathscr{F}_{t}\right] \\
& =B_{t}^{\mathrm{d}}\left(T_{j}^{k}\right) \mathbb{E}_{P_{T_{j}^{k}}^{\mathrm{d}}}\left[\left(\eta_{j}^{k} \exp \left(-X_{j}^{k}\right)-\tilde{K}^{k}\right) \mid \mathscr{F}_{t}\right],
\end{aligned}
$$

where we set

$$
\eta_{j}^{k}:=\frac{1}{D_{j}^{k}}=\frac{B_{0}^{\mathrm{d}}\left(T_{j-1}^{k}\right)}{B_{0}^{\mathrm{d}}\left(T_{j}^{k}\right)} \exp \left(\int_{0}^{T_{j-1}^{k}}\left[A^{k}\left(s, T_{j}^{k}\right)-A^{k}\left(s, T_{j-1}^{k}\right)\right] d s\right)
$$

and $\tilde{K}^{k}:=1+\delta^{k} K$ (cf. equation 2.14). The time- $t$ FRA rate $K_{t}^{k}$ is given by

$$
K_{t}^{k}=\mathbb{E}_{P_{T_{j}^{\mathrm{d}}}^{\mathrm{d}}}\left[\frac{1}{\delta^{k}}\left(B_{T_{j-1}^{k}}^{k}\left(T_{j}^{k}\right)^{-1}-1\right) \mid \mathscr{F}_{t}\right]=\mathbb{E}_{P_{T_{j}^{k}}^{\mathrm{d}}}\left[\frac{1}{\delta^{k}}\left(\eta_{j}^{k} \exp \left(-X_{j}^{k}\right)-1\right) \mid \mathscr{F}_{t}\right] .
$$

To obtain a tractable representation of the price, we consider the following term:

$$
\begin{aligned}
& \mathbb{E}_{P_{T_{j}^{\mathrm{d}}}^{\mathrm{d}}}\left[B_{T_{j-1}^{k}}^{k}\left(T_{j}^{k}\right)^{-1} \mid \mathscr{F}_{t}\right]=\eta_{j}^{k} \mathbb{E}_{P_{T_{j}^{\mathrm{d}}}^{\mathrm{d}}}\left[\exp \left(-\int_{0}^{T_{j-1}^{k}} \Sigma^{k}\left(s, T_{j-1}^{k}, T_{j}^{k}\right) d L_{s}\right) \mid \mathscr{F}_{t}\right] \\
& =\eta_{j}^{k} \exp \left(-\int_{0}^{t} \Sigma^{k}\left(s, T_{j-1}^{k}, T_{j}^{k}\right) d L_{s}\right) \mathbb{E}_{P_{T_{j}^{k}}^{\mathrm{d}}}\left[\exp \left(-\int_{t}^{T_{j-1}^{k}} \Sigma^{k}\left(s, T_{j-1}^{k}, T_{j}^{k}\right) d L_{s}\right)\right] \\
& =\eta_{j}^{k} \exp \left(-\int_{0}^{t} \Sigma^{k}\left(s, T_{j-1}^{k}, T_{j}^{k}\right) d L_{s}+\int_{t}^{T_{j-1}^{k}} \theta_{s}^{T_{j}^{k}}\left(-\Sigma^{k}\left(s, T_{j-1}^{k}, T_{j}^{k}\right)\right) d s\right)
\end{aligned}
$$

where $\theta_{t}^{T_{j}^{k}}$ denotes the cumulant of $L$ under $P_{T_{j}^{k}}^{\mathrm{d}}$.

Proposition 3.3.1 The risk-neutral price of a forward rate agreement at time $t$ can be written as

$$
\operatorname{FRA}_{t}\left(T_{j-1}^{k}, T_{j}^{k}, \delta^{k}, K\right)=B_{t}^{\mathrm{d}}\left(T_{j}^{k}\right)\left(V_{t}^{k}\left(T_{j-1}^{k}, T_{j}^{k}\right)-\tilde{K}^{k}\right),
$$


where we define

$$
\begin{aligned}
V_{t}^{k}\left(T_{j-1}^{k}, T_{j}^{k}\right):= & \eta_{j}^{k} \exp \left(-\int_{0}^{t} \Sigma^{k}\left(s, T_{j-1}^{k}, T_{j}^{k}\right) d L_{s}\right) \\
& \times \exp \left(\int_{t}^{T_{j-1}^{k}}\left[\theta_{s}\left(\Sigma^{\mathrm{d}}\left(s, T_{j}^{k}\right)-\Sigma^{k}\left(s, T_{j-1}^{k}, T_{j}^{k}\right)\right)-\theta_{s}\left(\Sigma^{\mathrm{d}}\left(s, T_{j}^{k}\right)\right)\right] d s\right) .
\end{aligned}
$$

The rate $K_{t}^{k}$ such that

$$
\operatorname{FRA}_{t}\left(T_{j-1}^{k}, T_{j}^{k}, \delta^{k}, K_{t}^{k}\right)=0
$$

is given by

$$
K_{t}^{k}=\frac{1}{\delta^{k}}\left(V_{t}^{k}\left(T_{j-1}^{k}, T_{j}^{k}\right)-1\right) .
$$

Proof: Given the formula (3.41) and the analysis made before this Proposition, it remains to show that

$$
\begin{aligned}
& \mathbb{E}_{P_{T_{j}^{\mathrm{d}}}^{k}}\left[\exp \left(-\int_{t}^{T_{j-1}^{k}} \Sigma^{k}\left(s, T_{j-1}^{k}, T_{j}^{k}\right) d L_{s}\right)\right] \\
= & \exp \left(-\int_{0}^{T_{j-1}^{k}} A^{\mathrm{d}}\left(s, T_{j}^{k}\right) d s\right) \mathbb{E}_{P^{\mathrm{d}}}\left[\operatorname { e x p } \left(\int_{0}^{T_{j-1}^{k}} \Sigma^{\mathrm{d}}\left(s, T_{j}^{k}\right) d L_{s}\right.\right. \\
& \left.\left.-\int_{t}^{T_{j-1}^{k}} \Sigma^{k}\left(s, T_{j-1}^{k}, T_{j}^{k}\right) d L_{s}\right)\right] \\
= & \exp \left(-\int_{0}^{T_{j-1}^{k}} \theta_{s}\left(\Sigma^{\mathrm{d}}\left(s, T_{j}^{k}\right)\right) d s\right) \mathbb{E}_{P_{\mathrm{d}}^{\mathrm{d}}}\left[\exp \left(\int_{0}^{t} \Sigma^{\mathrm{d}}\left(s, T_{j}^{k}\right) d L_{s}\right)\right] \\
& \times \mathbb{E}_{P_{\mathrm{d}}^{\mathrm{d}}}\left[\exp \left(\int_{t}^{T_{j-1}^{k}} \Sigma^{\mathrm{d}}\left(s, T_{j}^{k}\right)-\Sigma^{k}\left(s, T_{j-1}^{k}, T_{j}^{k}\right) d L_{s}\right)\right] \\
= & \exp \left(-\int_{0}^{T_{j-1}^{k}} \theta_{s}\left(\Sigma^{\mathrm{d}}\left(s, T_{j}^{k}\right)\right) d s\right) \exp \left(\int_{0}^{t} \theta_{s}\left(\Sigma^{\mathrm{d}}\left(s, T_{j}^{k}\right)\right) d s\right) \\
& \times \exp \left(\int_{t}^{T_{j-1}^{k}} \theta_{s}\left(\Sigma^{\mathrm{d}}\left(s, T_{j}^{k}\right)-\Sigma^{k}\left(s, T_{j-1}^{k}, T_{j}^{k}\right)\right) d s\right) \\
= & \exp \left(\int_{t}^{T_{j-1}^{k}}\left[\theta_{s}\left(\Sigma^{\mathrm{d}}\left(s, T_{j}^{k}\right)-\Sigma^{k}\left(s, T_{j-1}^{k}, T_{j}^{k}\right)\right)-\theta_{s}\left(\Sigma^{\mathrm{d}}\left(s, T_{j}^{k}\right)\right)\right] d s\right) .
\end{aligned}
$$




\section{Interest Rate Swaps}

The time- $t$ value of an interest rate swap with fixing and payment dates $\mathscr{T}^{k}$ can be computed as

$$
\begin{aligned}
\operatorname{Swap}_{t}\left(\mathscr{T}^{k}, \delta^{k}, \mathrm{~S}\right) & =\sum_{j=1}^{n_{k}} \delta^{k} B_{t}^{\mathrm{d}}\left(T_{j}^{k}\right) \mathbb{E}_{P_{T_{j}^{k}}^{\mathrm{d}}}\left[\left(L_{T_{j-1}^{k}}^{k}\left(T_{j-1}^{k}, T_{j}^{k}\right)-\mathrm{S}\right) \mid \mathscr{F}_{t}\right] \\
& =\sum_{j=1}^{n_{k}} \operatorname{FRA}_{t}\left(T_{j-1}^{k}, T_{j}^{k}, \delta^{k}, \mathrm{~S}\right) \\
& =\sum_{j=1}^{n_{k}} B_{t}^{\mathrm{d}}\left(T_{j}^{k}\right)\left(V_{t}^{k}\left(T_{j-1}^{k}, T_{j}^{k}\right)-\tilde{\mathrm{S}}^{k}\right)
\end{aligned}
$$

where we define $\tilde{S}^{k}=1+\delta^{k} S$ (cf. relation 2.17). The swap rate at time $t$ with $t \leq T_{0}^{k}$, denoted by $\mathrm{S}_{t}^{k}\left(\mathscr{T}^{k}\right)$, is defined as the rate that makes the time- $t$ value of the swap equal to zero. We therefore obtain

$$
\mathrm{S}_{t}^{k}\left(\mathscr{T}^{k}\right)=\frac{\sum_{j=1}^{n_{k}} B_{t}^{\mathrm{d}}\left(T_{j}^{k}\right)\left(V_{t}^{k}\left(T_{j-1}^{k}, T_{j}^{k}\right)-1\right)}{\sum_{j=1}^{n_{k}} \delta^{k} B_{t}^{\mathrm{d}}\left(T_{j}^{k}\right)} .
$$

The Libor/Euribor-OIS swap spread is given by

$$
\mathrm{S}_{t}^{k}\left(\mathscr{T}^{k}\right)-\mathrm{S}_{t}^{\text {on }}\left(\mathscr{T}^{k}\right)=\frac{\sum_{j=1}^{n_{k}} B_{t}^{\mathrm{d}}\left(T_{j}^{k}\right)\left(V_{t}^{k}\left(T_{j-1}^{k}, T_{j}^{k}\right)-1\right)-B_{t}^{\mathrm{d}}\left(T_{0}^{k}\right)+B_{t}^{\mathrm{d}}\left(T_{n_{k}}^{k}\right)}{\sum_{j=1}^{n_{k}} \delta^{k} B_{t}^{\mathrm{d}}\left(T_{j}^{k}\right)} .
$$

\section{Basis Swaps}

Let us consider $k \leq l$. From valuation formula (2.18), we immediately obtain that

$$
\mathrm{BS}_{t}^{1}\left(\mathscr{T}^{k}, \mathscr{T}^{l}\right)=\sum_{j=1}^{n_{l}} B_{t}^{\mathrm{d}}\left(T_{j}^{l}\right)\left(V_{t}^{l}\left(T_{j-1}^{l}, T_{j}^{l}\right)-1\right)-\sum_{j=1}^{n_{k}} B_{t}^{\mathrm{d}}\left(T_{j}^{k}\right)\left(V_{t}^{k}\left(T_{j-1}^{k}, T_{j}^{k}\right)-1\right) .
$$

Moreover, it results from equation 2.19 that

$$
\begin{aligned}
\mathrm{BS}_{t}^{2}\left(\mathscr{T}^{k}, \mathscr{T}^{l}\right)= & \sum_{j=1}^{n_{l}} B_{t}^{\mathrm{d}}\left(T_{j}^{l}\right)\left(V_{t}^{l}\left(T_{j-1}^{l}, T_{j}^{l}\right)-1\right)-\sum_{j=1}^{n_{k}} B_{t}^{\mathrm{d}}\left(T_{j}^{k}\right)\left(V_{t}^{k}\left(T_{j-1}^{k}, T_{j}^{k}\right)-1\right) \\
& -\sum_{k=1}^{n} \delta B_{t}^{\mathrm{d}}\left(T_{k}\right) \cdot \mathrm{bs}_{t}
\end{aligned}
$$


and

$$
\mathrm{bs}_{t}=\frac{\sum_{j=1}^{n_{l}} B_{t}^{\mathrm{d}}\left(T_{j}^{l}\right)\left(V_{t}^{l}\left(T_{j-1}^{l}, T_{j}^{l}\right)-1\right)-\sum_{j=1}^{n_{k}} B_{t}^{\mathrm{d}}\left(T_{j}^{k}\right)\left(V_{t}^{k}\left(T_{j-1}^{k}, T_{j}^{k}\right)-1\right)}{\sum_{k=1}^{n} \delta B_{t}^{\mathrm{d}}\left(T_{k}\right)} .
$$

\section{Caps and Floors}

Given the pricing formula 2.20 , the time- $t$ value of a cap with strike rate $K$ and maturity $T^{*}$ results in

$$
\begin{aligned}
\operatorname{Cap}_{t}\left(\mathscr{T}^{k}, \delta^{k}, K\right) & =\sum_{j=1}^{n_{k}}\left(\beta_{t}^{\mathrm{d}}\right)^{-1} \mathbb{E}_{P^{\mathrm{d}}}\left[\beta_{T_{j}^{k}}^{\mathrm{d}}\left(B_{T_{j-1}^{k}}^{k}\left(T_{j}^{k}\right)^{-1}-\tilde{K}^{k}\right)^{+} \mid \mathscr{F}_{t}\right] \\
& =\sum_{j=1}^{n_{k}}\left(\beta_{t}^{\mathrm{d}}\right)^{-1} \mathbb{E}_{P^{\mathrm{d}}}\left[\beta_{T_{j}^{k}}^{\mathrm{d}}\left(\eta_{j}^{k} \exp \left(-X_{j}^{k}\right)-\tilde{K}^{k}\right)^{+} \mid \mathscr{F}_{t}\right] \\
& =\sum_{j=1}^{n_{k}} \mathbb{E}_{P_{T_{j}^{k}}^{\mathrm{d}}}\left[B_{t}^{\mathrm{d}}\left(T_{j}^{k}\right)\left(\eta_{j}^{k} \exp \left(-X_{j}^{k}\right)-\tilde{K}^{k}\right)^{+} \mid \mathscr{F}_{t}\right] .
\end{aligned}
$$

Hence, the time- 0 price of a caplet with strike rate $K$ is given by

$$
\begin{aligned}
\mathrm{Cpl}_{0}\left(T_{j-1}^{k}, T_{j}^{k}, K\right) & =\mathbb{E}_{P^{\mathrm{d}}}\left[\beta_{T_{j}^{k}}^{\mathrm{d}}\left(\eta_{j}^{k} \exp \left(-X_{j}^{k}\right)-\tilde{K}^{k}\right)^{+}\right] \\
& =\mathbb{E}_{P_{T_{j}^{\mathrm{d}}}^{\mathrm{d}}}\left[B_{0}^{\mathrm{d}}\left(T_{j}^{k}\right)\left(\eta_{j}^{k} \exp \left(-X_{j}^{k}\right)-\tilde{K}^{k}\right)^{+}\right] .
\end{aligned}
$$

To simplify the notation, we set $Y_{j}^{k}:=-X_{j}^{k}$. The extended characteristic function of $Y_{j}^{k}$ relative to pricing measure $P_{T_{j}^{k}}^{\mathrm{d}}$ can be calculated as

$$
\begin{aligned}
\varphi_{Y_{j}^{k}}^{T_{j}^{k}}(z) & =\mathbb{E}_{P_{T_{j}^{\mathrm{d}}}^{k}}\left[\exp \left(\int_{0}^{T_{j-1}^{k}}-i z \Sigma^{k}\left(s, T_{j-1}^{k}, T_{j}^{k}\right) d L_{s}\right)\right] \\
& =\exp \left(\int_{0}^{T_{j-1}^{k}}\left[\theta_{s}\left(\Sigma^{\mathrm{d}}\left(s, T_{j}^{k}\right)-i z \Sigma^{k}\left(s, T_{j-1}^{k}, T_{j}^{k}\right)\right)-\theta_{s}\left(\Sigma^{\mathrm{d}}\left(s, T_{j}^{k}\right)\right)\right] d s\right)
\end{aligned}
$$

for every $z \in \mathbb{C}$ where this function exists.

To evaluate the price of the caplet, we use the Fourier based valuation method (see Eberlein, Glau, and Papapantoleon [40]). 
Proposition 3.3.2 The risk-neutral price of the caplet at time 0 can be written as

$$
\mathrm{Cpl}_{0}\left(T_{j-1}^{k}, T_{j}^{k}, K\right)=B_{0}^{\mathrm{d}}\left(T_{j}^{k}\right) \frac{e^{-R \xi_{j}^{k}}}{\pi} \int_{0}^{\infty} \operatorname{Re}\left(\frac{e^{-i u \xi_{j}^{k}} \varphi_{Y_{j}^{k}}^{T_{j}^{k}}(u-i R)\left(\tilde{K}^{k}\right)^{1-R-i u}}{(-R-i u)(1-R-i u)}\right) d u
$$

for any $R \in\left(1, \frac{M-\hat{M}}{\hat{M}}\right]$, where we set $\xi_{j}^{k}:=-\ln \eta_{j}^{k}$ and $\hat{M}$ is assumed to be chosen such that $\hat{M}<\frac{M}{2} . M$ is the constant in assumption $(\mathbb{E M})$ with respect to the driving process $L$.

Proof: Clearly, we have

$$
\begin{aligned}
\varphi_{Y_{j}^{k}}^{T_{j}^{k}}(u-i R)=\exp ( & \int_{0}^{T_{j-1}^{k}}\left[\theta_{s}\left(\Sigma^{\mathrm{d}}\left(s, T_{j}^{k}\right)-(i u+R) \Sigma^{k}\left(s, T_{j-1}^{k}, T_{j}^{k}\right)\right)\right. \\
& \left.\left.-\theta_{s}\left(\Sigma^{\mathrm{d}}\left(s, T_{j}^{k}\right)\right)\right] d s\right)
\end{aligned}
$$

and

$$
\begin{aligned}
\left|\operatorname{Re}\left(\Sigma_{l}^{\mathrm{d}}\left(s, T_{j}^{k}\right)-(i u+R) \sum_{l}^{k}\left(s, T_{j-1}^{k}, T_{j}^{k}\right)\right)\right| & =\left|\Sigma_{l}^{\mathrm{d}}\left(s, T_{j}^{k}\right)-R \Sigma_{l}^{k}\left(s, T_{j-1}^{k}, T_{j}^{k}\right)\right| \\
& \leq \hat{M}+|R| \hat{M} \leq \hat{M}+\frac{M-\hat{M}}{\hat{M}} \hat{M} \\
& =M
\end{aligned}
$$

for every $l \in\{1, \ldots, d\}$. Then, by Eberlein, Glau, and Papapantoleon [40, Theorem 2.2.] and an obvious symmetry property of the integrand, we prove the statement right.

The price of a floorlet can be obtained in an analogous way.

We temporarily consider the case $m=0$. Through a direct application of the Fourier pricing method, we obtain

$$
\begin{aligned}
& \operatorname{Cpl}_{0}\left(T_{j-1}, T_{j}, K\right)= \\
& =B_{0}^{\mathrm{d}}\left(T_{j-1}\right) \tilde{K} \frac{e^{-R_{1} \xi_{j}}}{\pi} \int_{0}^{\infty} \operatorname{Re}\left(e^{-i u \xi_{j}} \varphi_{X_{j}^{\mathrm{d}}}^{T_{j-1}}\left(u-i R_{1}\right) \frac{\tilde{K}^{-\left(1-R_{1}-i u\right)}}{\left(-R_{1}-i u\right)\left(1-R_{1}-i u\right)}\right) d u
\end{aligned}
$$

and

$$
\begin{aligned}
& \mathrm{Flt}_{0}\left(T_{j-1}, T_{j}, K\right)= \\
& =B_{0}^{\mathrm{d}}\left(T_{j-1}\right) \tilde{K} \frac{e^{-R_{2} \xi_{j}}}{\pi} \int_{0}^{\infty} \operatorname{Re}\left(e^{-i u \xi_{j}} \varphi_{X_{j}^{\mathrm{d}}}^{T_{j-1}}\left(u-i R_{2}\right) \frac{\tilde{K}^{-\left(1-R_{2}-i u\right)}}{\left(-R_{2}-i u\right)\left(1-R_{2}-i u\right)}\right) d u
\end{aligned}
$$


with $R_{1} \in\left[-\frac{M-\hat{M}}{\hat{M}}, 0\right)$ and $R_{2} \in\left(1,1+\frac{M-\hat{M}}{\hat{M}}\right]$ for a suitable $\hat{M}$ satisfying $\hat{M}<M$ and $\xi_{j}:=-\ln D_{j}^{\text {d. }}$. Here we use the fact that a caplet (floorlet) can be represented as a put (call) option on a bond.

The implementation of numerically efficient algorithms to compute the pricing formulas (3.45) and (3.46) allows to calibrate the model to market data (first step of the calibration procedure 2.4). This is achieved by our model (see chapter 4). Note that from the form of the caplets (floorlets) we immediately deduce the valuation formula of the cap (floor) price.

\section{Digital Options}

From formula $(2.22)$, we get the time- $T$ price of a delayed digital option as

$$
\mathrm{DD}_{T}^{k}\left(T_{j-1}^{k}, T_{j}^{k}, T, B, w\right)=\mathbb{1}_{\left\{w L_{T_{j-1}^{k}}^{k}\left(T_{j-1}^{k}, T_{j}^{k}\right)>w B\right\}},
$$

where we have $0<B, T_{j-1}^{k} \leq T$ and

$$
w=\left\{\begin{array}{l}
1, \text { for a delayed digital call } \\
-1, \text { for a delayed digital put }
\end{array}\right.
$$

Then, the time- $t$ price $\left(t \leq T_{j-1}^{k}\right)$ can be represented by

$$
\begin{aligned}
\mathrm{DD}_{t}^{k}\left(T_{j-1}^{k}, T_{j}^{k}, T, B, w\right) & =\left(\beta_{t}^{\mathrm{d}}\right)^{-1} \mathbb{E}_{P^{\mathrm{d}}}\left[\beta_{T}^{\mathrm{d}} \mathbb{1}_{\left\{w L_{T_{j-1}^{k}}^{k}\left(T_{j-1}^{k}, T_{j}^{k}\right)>w B\right\}} \mid \mathscr{F}_{t}\right] \\
& =B_{t}^{\mathrm{d}}(T) \mathbb{E}_{P_{T}^{\mathrm{d}}}\left[\mathbb{1}_{\left\{w L_{T_{j-1}^{k}}^{k}\left(T_{j-1}^{k}, T_{j}^{k}\right)>w B\right\}} \mid \mathscr{F}_{t}\right] \\
& =B_{t}^{\mathrm{d}}(T) \mathbb{E}_{P_{T}^{\mathrm{d}}}\left[\mathbb{1}_{\left\{w\left(1+\delta^{k} B\right)^{-1}>w B_{T_{j-1}^{k}}^{k}\left(T_{j}^{k}\right)\right\}} \mid \mathscr{F}_{t}\right] .
\end{aligned}
$$

This formula can further be written as

$$
\mathrm{DD}_{t}^{k}\left(T_{j-1}^{k}, T_{j}^{k}, T, B, w\right)=B_{t}^{\mathrm{d}}(T) \mathbb{E}_{P_{T}^{\mathrm{d}}}\left[\mathbb{1}_{\left\{w\left(1+\delta^{k} B\right)^{-1}>w B_{t}^{k}\left(T_{j}^{k}\right) B_{t}^{k}\left(T_{j-1}^{k}\right)^{-1} H^{k}\left(t, T_{j-1}^{k}\right)\right\}} \mid \mathscr{F}_{t}\right]
$$

with

$$
H^{k}\left(t, T_{j-1}^{k}\right):=\exp \left(-\int_{t}^{T_{j-1}^{k}} A^{k}\left(s, T_{j-1}^{k}, T_{j}^{k}\right) d s+X_{t, j}^{k}\right),
$$

where we set

$$
A^{k}\left(s, T_{j-1}^{k}, T_{j}^{k}\right):=A^{k}\left(s, T_{j}^{k}\right)-A^{k}\left(s, T_{j-1}^{k}\right)
$$


3. The Multiple-Curve Lévy Forward Rate Model with Application of the Two-Price Theory

and

$$
X_{t, j}^{k}:=\int_{t}^{T_{j-1}^{k}} \Sigma^{k}\left(s, T_{j-1}^{k}, T_{j}^{k}\right) d L_{s}
$$

By the independence of $X_{t, j}^{k}$ and $\mathscr{F}_{t}$ (independent increments of the process $L$ ) and the $\mathscr{F}_{t}$-measurability of $\frac{B_{t}^{k}\left(T_{j}^{k}\right)}{B_{t}^{k}\left(T_{j-1}^{k}\right)}$, we obtain from Kallenberg [60, Theorem 6.4] that

$$
\begin{aligned}
\operatorname{DD}_{t}^{k}\left(T_{j-1}^{k}, T_{j}^{k}, T, B, w\right) & =B_{t}^{\mathrm{d}}(T) \cdot g_{w}^{k}\left(\frac{B_{t}^{k}\left(T_{j}^{k}\right)}{B_{t}^{k}\left(T_{j-1}^{k}\right)}\right) \\
& =B_{t}^{\mathrm{d}}(T) \cdot g_{w}^{k}\left(F^{k}\left(t, T_{j-1}^{k}, T_{j}^{k}\right)^{-1}\right)
\end{aligned}
$$

where $F^{k}\left(t, T_{j-1}^{k}, T_{j}^{k}\right)=\frac{B_{t}^{k}\left(T_{j-1}^{k}\right)}{B_{t}^{k}\left(T_{j}^{k}\right)}$ and the function $g_{w}^{k}: \mathbb{R} \rightarrow[0,1]$ is defined by

$$
g_{w}^{k}(y):=\mathbb{E}_{P_{T}^{\mathrm{d}}}\left[\mathbb{1}_{\left\{w\left(1+\delta^{k} B\right)^{-1}>w y H^{k}\left(t, T_{j-1}^{k}\right)\right\}}\right] .
$$

For every $y>0$, we have

$$
\begin{aligned}
& g_{w}^{k}(y)=P_{T}^{\mathrm{d}}\left(w \exp \left(X_{t, j}^{k}\right)<w \frac{\exp \left(\int_{t}^{T_{j-1}^{k}} A^{k}\left(s, T_{j-1}^{k}, T_{j}^{k}\right) d s\right)}{\left(1+\delta^{k} B\right) y}\right) \\
& =P_{T}^{\mathrm{d}, X_{t, j}^{k}}\left(w \exp (x)<w \frac{\exp \left(\int_{t}^{T_{j-1}^{k}} A^{k}\left(s, T_{j-1}^{k}, T_{j}^{k}\right) d s\right)}{\left(1+\delta^{k} B\right) y}\right) \\
& = \begin{cases}P_{T}^{\mathrm{d}, X_{t, j}^{k}}\left(x<\log \left(\frac{\exp \left(\int_{t}^{T_{j-1}^{k}} A^{k}\left(s, T_{j-1}^{k}, T_{j}^{k}\right) d s\right.}{\left(1+\delta^{k} B\right) y}\right)\right), & \text { for } w=1 \\
1-P_{T}^{\mathrm{d}, X_{t, j}^{k}}\left(x \leq \log \left(\frac{\exp \left(\int_{t}^{T_{j-1}^{k}} A^{k}\left(s, T_{j-1}^{k}, T_{j}^{k}\right) d s\right)}{\left(1+\delta^{k} B\right) y}\right)\right), & \text { for } w=-1,\end{cases}
\end{aligned}
$$


where we denote by $P_{T}^{\mathrm{d}, X_{t, j}^{k}}$ the distribution of $X_{t, j}^{k}$. The extended characteristic function of $X_{t, j}^{k}$ under $P_{T}^{\mathrm{d}}$ can be determined by

$$
\varphi_{X_{t, j}^{k}}^{T}(z)=\exp \left(\int_{t}^{T_{j-1}^{k}} \theta_{s}\left(\Sigma^{\mathrm{d}}(s, T)+i z \Sigma^{k}\left(s, T_{j-1}^{k}, T_{j}^{k}\right)\right)-\theta_{s}\left(\Sigma^{\mathrm{d}}(s, T)\right) d s\right)
$$

for every $z \in \mathbb{C}$ where this function is defined.

Now, we calculate the present value $(t=0)$ of a delayed digital option by applying the Fourier based valuation method developed by Eberlein, Glau, and Papapantoleon [40, Theorem 2.7.]. To this end, let us write the value of the delayed digital option at time $t=0$ as

$$
\begin{aligned}
& \operatorname{DD}_{0}^{k}\left(T_{j-1}^{k}, T_{j}^{k}, T, B, w\right) \\
& =B_{0}^{\mathrm{d}}(T) \mathbb{E}_{P_{T}^{\mathrm{d}}}\left[\mathbb{1}_{\left\{w \exp \left(X_{j}^{k}-\xi_{j}^{k}\right)<w\left(1+\delta^{k} B\right)^{-1}\right\}}\right] \\
& =B_{0}^{\mathrm{d}}(T) P_{T}^{\mathrm{d}}\left(w \exp \left(X_{j}^{k}-\xi_{j}^{k}\right)<w\left(1+\delta^{k} B\right)^{-1}\right) \\
& =\left\{\begin{array}{l}
B_{0}^{\mathrm{d}}(T) P_{T}^{\mathrm{d}}\left(X_{j}^{k}<\log \left(\left(1+\delta^{k} B\right)^{-1}\right)+\xi_{j}^{k}\right), \text { for } w=1 \\
B_{0}^{\mathrm{d}}(T) P_{T}^{\mathrm{d}}\left(X_{j}^{k}>\log \left(\left(1+\delta^{k} B\right)^{-1}\right)+\xi_{j}^{k}\right), \text { for } w=-1
\end{array}\right. \\
& =\left\{\begin{array}{l}
B_{0}^{\mathrm{d}}(T) P_{T}^{\mathrm{d}}\left(X_{j}^{k}<\log \left(\left(1+\delta^{k} B\right)^{-1}\right)+\xi_{j}^{k}\right), \text { for } w=1 \\
B_{0}^{\mathrm{d}}(T)\left[1-F_{X_{j}^{k}}^{T}\left(\log \left(\left(1+\delta^{k} B\right)^{-1}\right)+\xi_{j}^{k}\right)\right], \text { for } w=-1
\end{array}\right. \\
& =: V_{w}^{j, k}\left(\xi_{j}^{k}\right),
\end{aligned}
$$

where we obtain $\xi_{j}^{k}=-\log \left(D_{j}^{k}\right) \in \mathbb{R}$ and $F_{X}^{T}$ denotes the cumulative distribution function of a random variable $X$ under $P_{T}^{\mathrm{d}}$. Let us consider $V_{w}^{j, k}$ as the value of the delayed digital option comprehended as function of $\xi_{j}^{k}$, i.e. we define the map $\mathbb{R} \ni \xi_{j}^{k} \mapsto$ $V_{w}^{j, k}\left(\xi_{j}^{k}\right) \in \mathbb{R}_{+}$. Clearly, $V_{w}^{j, k}$ has locally bounded variation. We assume that the distribution of $X_{j}^{k}$ under $P_{T}^{\mathrm{d}}$ is atomless (see Elstrodt [43]). Then, it holds

$$
V_{w}^{j, k}\left(\xi_{j}^{k}\right)= \begin{cases}B_{0}^{\mathrm{d}}(T) F_{X_{j}^{k}}^{T}\left(\left(1+\delta^{k} B\right)^{-1}+\xi_{j}^{k}\right), & \text { for } w=1 \\ B_{0}^{\mathrm{d}}(T)\left(1-F_{X_{j}^{k}}^{T}\left(\left(1+\delta^{k} B\right)^{-1}+\xi_{j}^{k}\right)\right), & \text { for } w=-1 .\end{cases}
$$

It follows that $V_{w}^{j, k}$ is a continuous function. The payoff function of a digital call option with barrier $B \in \mathbb{R}_{+}$is given by

$$
f_{w}(x)=\mathbb{1}_{\left\{w e^{x}<w \tilde{B}_{k}^{-1}\right\}},
$$

where we set $\tilde{B}_{k}=1+\delta^{k} B$. Easy calculations lead to the following form of the Fourier 
transform of $f_{w}$ for every $z \in \mathbb{C}$ where it is defined:

$$
\hat{f}_{w}(z)= \begin{cases}\frac{\tilde{B}_{k}^{-i z}}{i z}, & \text { for } w=1 \text { and } \operatorname{Im}(z) \in(-\infty, 0) \\ -\frac{\tilde{B}_{k}^{-i z}}{i z}, & \text { for } w=-1 \text { and } \operatorname{Im}(z) \in(0, \infty) .\end{cases}
$$

Let us denote the dampened payoff function of the digital option by $d_{w}$. Recall that it is given by $d_{w}(x)=e^{-R x} f_{w}(x)$. We easily verify that $d_{w} \in L^{1}(\mathbb{R})$ for every

$$
\begin{cases}R \in(-\infty, 0), & \text { for } w=1 \\ R \in(0, \infty), & \text { for } w=-1\end{cases}
$$

Observe that we can find an $R$ that satisfies the prerequisites of Eberlein, Glau, and Papapantoleon [40, Theorem 2.7.]. Then, we conclude from this Theorem that the value of a delayed digital option at point $\xi_{j}^{k}$ can be expressed as

$$
\begin{aligned}
& \operatorname{DD}_{0}^{k}\left(T_{j-1}^{k}, T_{j}^{k}, T, B, w\right) \\
& =\left\{\begin{array}{l}
B_{0}^{\mathrm{d}}(T) \cdot \lim _{A \rightarrow \infty} \frac{e^{-R \xi_{j}^{k}}}{\pi} \int_{0}^{A} \operatorname{Re}\left(\frac{e^{-i u \xi_{j}^{k}} \varphi_{X_{j}^{k}}^{T}(u-i R) \tilde{B}_{k}^{R+i u}}{-R-i u}\right) d u, \text { for } w=1 \text { and } R<0 \\
B_{0}^{\mathrm{d}}(T) \cdot \lim _{A \rightarrow \infty} \frac{e^{-R \xi_{j}^{k}}}{\pi} \int_{0}^{A} \operatorname{Re}\left(\frac{e^{-i u \xi_{j}^{k}} \varphi_{X_{j}^{k}}^{T}(u-i R) \tilde{B}_{k}^{R+i u}}{R+i u}\right) d u, \text { for } w=-1 \text { and } R>0 .
\end{array}\right.
\end{aligned}
$$

Note that the price of a delayed range digital option with barriers $\underline{B}, \bar{B}$ satisfying $0<$ $\underline{B}<\bar{B}$ can determined by formula 2.23 .

\subsubsection{Application of the Two-Price Theory}

Now, the theory of two prices is applied to our multiple-curve model. We provide ask and bid valuation formulas for some special interest rate derivatives. Let us consider $m \in \mathbb{N}$ and an equidistant discrete tenor structure $\mathscr{T}^{k}=\left\{T_{0}^{k}, \ldots, T_{n_{k}}^{k}\right\}$ with tenor $\delta^{k}$ and $k \in\{0,1, \ldots, m\}$. As before, instead of writing 0 for the discount curve we also write d.

\section{Ask and Bid Price of Caplets and Floorlets}

Our first aim is to derive explicit valuation formulas of ask and bid prices of caplets and floorlets. According to the two-price theory in section 2.1, in order to determine these formulas we have to distort the cumulative distribution function of the discounted payoff

$$
\beta_{T_{j}^{k}}^{\mathrm{d}}\left(\eta_{j}^{k} \exp \left(-X_{j}^{k}\right)-\tilde{K}^{k}\right)^{+}
$$


with respect to measure $P^{\mathrm{d}}$ (cf. relation 3.42 ). Although this can be done analytically, this calculation is, in general, a challenging task. In most cases, it has to be determined numerically and its numerical evaluation is extremely time-consuming. The main reason for this lies in the joint appearance of the random variables $\beta_{T_{j}^{k}}^{\mathrm{d}}$ and $X_{j}^{k}$ in 3.48 . To handle this issue, we switch to the forward martingale measure and consider the more tractable discounted payoff

$$
\mathrm{Cp}_{j, K}^{k}:=B_{0}^{\mathrm{d}}\left(T_{j}^{k}\right)\left(\eta_{j}^{k} \exp \left(-X_{j}^{k}\right)-\tilde{K}^{k}\right)^{+}
$$

(cf. pricing formula 3.43 and the procedure described in section 2.3). Analogously, we deal with the corresponding floorlets quantity

$$
\mathrm{FIt}_{j, K}^{k}:=B_{0}^{\mathrm{d}}\left(T_{j}^{k}\right)\left(\tilde{K}^{k}-\eta_{j}^{k} \exp \left(-X_{j}^{k}\right)\right)^{+} .
$$

Let us denote by $F_{Y}^{T}$ the cumulative distribution function of a random variable $Y$ under $P_{T}^{\mathrm{d}}$ with $T \in\left[0, T^{*}\right]$.

Lemma 3.3.1 1. Let us assume that $X_{j}^{k}$ has exponential moments of order $1<M_{j}^{k}$ under $P_{T_{j}^{k}}^{\mathrm{d}}$. Then, it results that

(i) for any $\gamma \geq 0$, we have

$$
a_{\gamma}^{T_{j}^{k}}\left(\mathrm{Cpl}_{j, K}^{k}\right)=\int_{-\infty}^{0} \Psi_{\gamma}^{\mathrm{mv}}\left(F_{-\mathrm{Cpl}}^{T_{j, K}^{k}}(x)\right) d x
$$

and

$$
b_{\gamma}^{T_{j}^{k}}\left(\mathrm{Cpl}_{j, K}^{k}\right)=\int_{0}^{\infty}\left(1-\Psi_{\gamma}^{\mathrm{mv}}\left(F_{\mathrm{Cpl}_{j, K}^{k}}^{T_{j}^{k}}(x)\right)\right) d x .
$$

(ii) for the the family $\Psi=\left(\Psi_{\gamma}\right)_{\gamma \geq 0} \in\left\{\Psi^{\text {mav }}, \Psi^{\mathrm{mmv}}, \Psi^{\mathrm{mamv}}\right\}$ of distortion functions and every $\gamma \in\left[0, u_{1}-1\right)$, we have

$$
a_{\gamma}^{T_{j}^{k}}\left(\mathrm{Cpl}_{j, K}^{k}\right)=\int_{-\infty}^{0} \Psi_{\gamma}\left(F_{-\mathrm{Cpl}}^{T_{j, K}^{k}}(x)\right) d x
$$

and

$$
b_{\gamma}^{T_{j}^{k}}\left(\mathrm{Cpl}_{j, K}^{k}\right)=\int_{0}^{\infty}\left(1-\Psi_{\gamma}\left(F_{\mathrm{Cpl}_{j, K}^{k}}^{T_{j}^{k}}(x)\right)\right) d x
$$

where we have $1<u_{1} \leq M_{j}^{k}$. 
3. The Multiple-Curve Lévy Forward Rate Model with Application of the Two-Price Theory

2. For every $\Psi=\left(\Psi_{\gamma}\right)_{\gamma \geq 0} \in\left\{\Psi^{\mathrm{mv}}, \Psi^{\mathrm{mav}}, \Psi^{\mathrm{mmv}}, \Psi^{\mathrm{mamv}}\right\}$ and $\gamma \geq 0$, we obtain

$$
a_{\gamma}^{P_{T_{j-1}^{k}}}\left(\mathrm{Flt}_{j, K}^{k}\right)=\int_{-\infty}^{0} \Psi_{\gamma}\left(F_{-\mathrm{Flt}_{j, K}^{k}}^{k}(x)\right) d x
$$

and

$$
b_{\gamma}^{P_{T_{j-1}^{k}}^{k}}\left(\mathrm{Flt}_{j, K}^{k}\right)=\int_{0}^{\infty}\left(1-\Psi_{\gamma}\left(F_{\mathrm{Flt}_{j, K}^{k}}(x)\right)\right) d x .
$$

Proof: We need to verify for which $\gamma \geq 0$ condition 2.10 is satisfied. We frequently use the change-of-variable formula here (see Billingsley [13, Theorem 16.13.]). Since $\mathrm{Cpl}_{j, K}^{k} \geq 0$, we only have to consider the second integral in 2.10 .

1. An application of Bernoulli's inequality leads to $\Psi_{\gamma}^{\mathrm{mv}}(y) \leq(1+\gamma) y$. For any $\gamma \geq 0$, we obtain

$$
\begin{aligned}
& \int_{0}^{\infty} \Psi_{\gamma}^{\mathrm{mv}}\left(1-F_{\mathrm{Cp}_{j, K}^{k}}(y)\right) d y \leq(1+\gamma) \int_{0}^{\infty} 1-F_{\mathrm{Cp}_{j, K}^{k}}(y) d y \\
& =(1+\gamma) B_{0}^{\mathrm{d}}\left(T_{j}^{k}\right) \int_{0}^{\infty} 1-F_{\left(\eta_{j}^{k} \exp \left(-X_{j}^{k}\right)-\tilde{K}^{k}\right)^{+}}^{T_{k}^{k}}(y) d y \\
& =(1+\gamma) B_{0}^{\mathrm{d}}\left(T_{j}^{k}\right) \int_{0}^{\infty} 1-F_{\eta_{j}^{k} \exp \left(-X_{j}^{k}\right)-\tilde{K}^{k}}^{T_{k}^{k}}(y) d y \\
& =(1+\gamma) B_{0}^{\mathrm{d}}\left(T_{j}^{k}\right) \int_{\tilde{K}^{k}}^{\infty} F_{X_{j}^{k}}^{T_{j}^{k}}\left(-\log \left(y D_{j}^{k}\right)\right) d y \\
& \leq(1+\gamma) B_{0}^{\mathrm{d}}\left(T_{j}^{k}\right) C_{1} \int_{\tilde{K}^{k}}^{\infty} e^{-u_{1} \log \left(y D_{j}^{k}\right)} d y \\
& =(1+\gamma) B_{0}^{\mathrm{d}}\left(T_{j}^{k}\right) C_{1} \int_{\tilde{K}^{k}}^{\infty}\left(y D_{j}^{k}\right)^{-u_{1}} d y<\infty,
\end{aligned}
$$

where we used Lemma 2.1 .1 with $C_{1}>0$ and $1<u_{1} \leq M_{j}^{k}$.

2. Since it holds

$$
\begin{aligned}
& \Psi_{\gamma}^{\mathrm{mamv}}(y)=\Psi_{\gamma}^{\mathrm{mav}}\left(\Psi_{\gamma}^{\mathrm{mv}}(y)\right) \leq \Psi_{\gamma}^{\mathrm{mav}}((1+\gamma) y)=(1+\gamma)^{\frac{1}{1+\gamma}} \Psi_{\gamma}^{\mathrm{mav}}(y) \\
& \Psi_{\gamma}^{\mathrm{mmv}}(y)=\Psi_{\gamma}^{\mathrm{mv}}\left(\Psi_{\gamma}^{\mathrm{mav}}(y)\right) \leq(1+\gamma) \Psi_{\gamma}^{\mathrm{mav}}(y),
\end{aligned}
$$

we only need to check the condition for the distortion function $\Psi^{\text {mav }}$. In a similar way as above, we get

$$
\int_{0}^{\infty} \Psi_{\gamma}^{\operatorname{mav}}\left(1-F_{\mathrm{Cpl}_{j, K}^{k}}(y)\right) d y \leq B_{0}^{\mathrm{d}}\left(T_{j}^{k}\right) C_{1}^{\frac{1}{1+\gamma}}\left(\eta_{j}^{k}\right)^{\frac{u_{1}}{1+\gamma}} \int_{\tilde{K}^{k}}^{\infty} y^{-\frac{u_{1}}{1+\gamma}} d y<\infty
$$

for every $\gamma$ satisfying $0 \leq \gamma<u_{1}-1$. 
3. Clearly, we have

$$
M_{\mathrm{Flt}_{j, K}^{k}}^{T_{j}^{k}}(u)=\mathbb{E}_{P_{T_{j}^{k}}^{\mathrm{d}}}\left[\exp \left(u \mathrm{Flt}_{j, K}^{k}\right)\right]<\exp \left(u B_{0}^{\mathrm{d}}\left(T_{j}^{k}\right) \tilde{K}^{k}\right)<\infty
$$

for every $u \in \mathbb{R}$. The claim follows through Proposition 2.1.2.

The pricing formulas immediately result from subsection 2.1.5.

It follows that

1. $\mathrm{Cpl}_{j, K}^{k}$ is regular with respect to $\alpha^{\mathrm{mv}}$,

2. $\mathrm{Cpl}_{j, K}^{k}$ is regular on the interval $\left[0, u_{1}-1\right)$ with respect to $\alpha^{\mathrm{mav}}, \alpha^{\mathrm{mmv}}$ and $\alpha^{\mathrm{mamv}}$ and

3. $\mathrm{Flt}_{j, K}^{k}$ is regular with respect to $\alpha^{\mathrm{mv}}, \alpha^{\mathrm{mav}}, \alpha^{\mathrm{mmv}}$ and $\alpha^{\mathrm{mamv}}$.

The following Proposition states useful integral representations for ask and bid prices of caplets and floorlets with reset date $T_{j-1}^{k}$, settlement date $T_{j}^{k}=T_{j-1}^{k}+\delta_{k}$ and strike rate $K$ at a permitted level $\gamma$.

Proposition 3.3.3 Let $\Psi=\left(\Psi_{x}\right)_{x \geq 0}$ be a proper family of distortion functions and $\gamma \geq 0$ be chosen such that condition (2.10) is satisfied for the caplet and floorlet. Then, the ask price of the caplet is given by

$$
a_{\gamma}^{T_{j}^{k}}\left(\mathrm{Cpl}_{j, K}^{k}\right)=B_{0}^{\mathrm{d}}\left(T_{j}^{k}\right) \int_{\tilde{K}^{k}}^{\infty} \Psi_{\gamma}\left(F_{X_{j}^{k}}^{T_{k}^{k}}\left(-\log \left(x D_{j}^{k}\right)\right)\right) d x
$$

and the bid price of the caplet has the form

$$
b_{\gamma}^{T_{j}^{k}}\left(\mathrm{Cpl}_{j, K}^{k}\right)=B_{0}^{\mathrm{d}}\left(T_{j}^{k}\right) \int_{\tilde{K}^{k}}^{\infty}\left[1-\Psi_{\gamma}\left(1-F_{X_{j}^{k}}^{T_{j}^{k}}\left(-\log \left(x D_{j}^{k}\right)\right)\right)\right] d x .
$$

The ask price of the floorlet is determined by

$$
a_{\gamma}^{T_{j}^{k}}\left(\mathrm{Flt}_{j, K}^{k}\right)=B_{0}^{\mathrm{d}}\left(T_{j}^{k}\right) \int_{0}^{\tilde{K}^{k}} \Psi_{\gamma}\left(1-F_{X_{j}^{k}}^{T_{j}^{k}}\left(-\log \left(x D_{j}^{k}\right)\right)\right) d x
$$

and the bid price of the floorlet is represented by

$$
b_{\gamma}^{T_{j}^{k}}\left(\mathrm{Flt}_{j, K}^{k}\right)=B_{0}^{\mathrm{d}}\left(T_{j}^{k}\right) \int_{0}^{\tilde{K}^{k}}\left[1-\Psi_{\gamma}\left(F_{X_{j}^{k}}^{T_{j}^{k}}\left(-\log \left(x D_{j}^{k}\right)\right)\right)\right] d x .
$$

Proof: We mainly apply the change-of-variable formula here (see Billingsley [13, Theorem 16.13.]). Then, we get the prices as: 
3. The Multiple-Curve Lévy Forward Rate Model with Application of the Two-Price Theory

1. Ask price:

$$
\begin{aligned}
& a_{\gamma}^{T_{j}^{k}}\left(\mathrm{Cpl}_{j, K}^{k}\right)=\int_{-\infty}^{0} \Psi_{\gamma}\left(F_{-\mathrm{Cpl} j_{j, K}^{k}}^{T_{j}^{k}}(x)\right) d x \\
& =\int_{-\infty}^{0} \Psi_{\gamma}\left(1-F_{j}^{T_{j}^{k}}\left(\eta_{j}^{k} \exp \left(-X_{j}^{k}\right)-\tilde{K}^{k}\right)^{+}\left(-x B_{0}^{\mathrm{d}}\left(T_{j}^{k}\right)^{-1}\right)\right) d x \\
& =B_{0}^{\mathrm{d}}\left(T_{j}^{k}\right) \int_{0}^{\infty} \Psi_{\gamma}\left(1-F_{\left(\eta_{j}^{k} \exp \left(-X_{j}^{k}\right)-\tilde{K}^{k}\right)^{+}}^{T_{j}^{k}}(x)\right) d x \\
& =B_{0}^{\mathrm{d}}\left(T_{j}^{k}\right) \int_{0}^{\infty} \Psi_{\gamma}\left(1-F_{\left(\eta_{j}^{k} \exp \left(-X_{j}^{k}\right)-\tilde{K}^{k}\right)}^{T_{j}^{k}}(x)\right) d x \\
& =B_{0}^{\mathrm{d}}\left(T_{j}^{k}\right) \int_{0}^{\infty} \Psi_{\gamma}\left(1-F_{\eta_{j}^{k} \exp \left(-X_{j}^{k}\right)}^{T_{k}^{k}}\left(x+\tilde{K}^{k}\right)\right) d x \\
& =B_{0}^{\mathrm{d}}\left(T_{j}^{k}\right) \int_{\tilde{K}^{k}}^{\infty} \Psi_{\gamma}\left(1-F_{\eta_{j}^{k}}^{T_{j}^{k}} \exp \left(-X_{j}^{k}\right)\right. \\
& =B_{0}^{\mathrm{d}}\left(T_{j}^{k}\right) \int_{\tilde{K}^{k}}^{\infty} \Psi_{\gamma}\left(1-F_{-X_{j}^{k}}^{T_{j}^{k}}\left(\log \left(x D_{j}^{k}\right)\right)\right) d x \\
& =B_{0}^{\mathrm{d}}\left(T_{j}^{k}\right) \int_{\tilde{K}^{k}}^{\infty} \Psi_{\gamma}\left(F_{X_{j}^{k}}^{T_{j}^{k}}\left(-\log \left(x D_{j}^{k}\right)\right)\right) d x
\end{aligned}
$$

2. Bid price:

$$
\begin{aligned}
& b_{\gamma}^{T_{j}^{k}}\left(\mathrm{Cpl}_{j, K}^{k}\right)=\int_{0}^{\infty}\left(1-\Psi_{\gamma}\left(F_{\mathrm{Cpl} j_{j, K}^{k}}^{T_{j}^{k}}(x)\right)\right) d x \\
& =\int_{0}^{\infty}\left(1-\Psi_{\gamma}\left(F_{\left(\eta_{j}^{k} \exp \left(-X_{j}^{k}\right)-\tilde{K}^{k}\right)^{+}}^{T_{j}^{k}}\left(x B_{0}^{\mathrm{d}}\left(T_{j}^{k}\right)^{-1}\right)\right)\right) d x \\
& =B_{0}^{\mathrm{d}}\left(T_{j}^{k}\right) \int_{0}^{\infty}\left(1-\Psi_{\gamma}\left(F_{\left(\eta_{j}^{k} \exp \left(-X_{j}^{k}\right)-\tilde{K}^{k}\right)^{+}}^{T^{k}}(x)\right)\right) d x \\
& =B_{0}^{\mathrm{d}}\left(T_{j}^{k}\right) \int_{0}^{\infty}\left(1-\Psi_{\gamma}\left(F_{\left(\eta_{j}^{k} \exp \left(-X_{j}^{k}\right)-\tilde{K}^{k}\right)}^{T_{k}^{k}}(x)\right)\right) d x \\
& =B_{0}^{\mathrm{d}}\left(T_{j}^{k}\right) \int_{0}^{\infty}\left(1-\Psi_{\gamma}\left(F_{\eta_{j}^{k} \exp \left(-X_{j}^{k}\right)}^{T_{k}^{k}}\left(x+\tilde{K}^{k}\right)\right)\right) d x \\
& =B_{0}^{\mathrm{d}}\left(T_{j}^{k}\right) \int_{\tilde{K}^{k}}^{\infty}\left(1-\Psi_{\gamma}\left(F_{-X_{j}^{k}}^{T_{j}^{k}}\left(\log \left(x D_{j}^{k}\right)\right)\right)\right) d x \\
& =B_{0}^{\mathrm{d}}\left(T_{j}^{k}\right) \int_{\tilde{K}^{k}}^{\infty}\left(1-\Psi_{\gamma}\left(1-F_{X_{j}^{k}}^{T_{j}^{k}}\left(-\log \left(x D_{j}^{k}\right)\right)\right)\right) d x
\end{aligned}
$$


1. Ask price:

$$
\begin{aligned}
& a_{\gamma}^{T_{j}^{k}}\left(\mathrm{Flt}{ }_{j, K}^{k}\right)=\int_{-\infty}^{0} \Psi_{\gamma}\left(F_{-\mathrm{Flt}_{j, K}^{k}}^{T_{j}^{k}}(x)\right) d x \\
& =\int_{-\infty}^{0} \Psi_{\gamma}\left(1-F_{\left(\tilde{K}^{k}-\eta_{j}^{k} \exp \left(-X_{j}^{k}\right)\right)^{+}}^{T_{k}^{k}}\left(-x B_{0}^{\mathrm{d}}\left(T_{j}^{k}\right)^{-1}\right)\right) d x \\
& =B_{0}^{\mathrm{d}}\left(T_{j}^{k}\right) \int_{0}^{\infty} \Psi_{\gamma}\left(1-F_{\left(\tilde{K}^{k}-\eta_{j}^{k} \exp \left(-X_{j}^{k}\right)\right)}^{T_{k}^{k}}(x)\right) d x \\
& =B_{0}^{\mathrm{d}}\left(T_{j}^{k}\right) \int_{0}^{\infty} \Psi_{\gamma}\left(1-F_{\left(\tilde{K}^{k}-\eta_{j}^{k} \exp \left(-X_{j}^{k}\right)\right)}^{T_{k}^{k}}(x)\right) d x \\
& =B_{0}^{\mathrm{d}}\left(T_{j}^{k}\right) \int_{0}^{\infty} \Psi_{\gamma}\left(F_{\eta_{j}^{k}}^{T^{k}} \exp \left(-X_{j}^{k}\right)\right. \\
& =B_{0}^{\mathrm{d}}\left(T_{j}^{k}\right) \int_{0}^{\tilde{K}^{k}} \Psi_{\gamma}\left(F_{\eta_{j}^{k}}^{T_{j}^{k}} \exp \left(-X_{j}^{k}\right)\right) d x \\
& =B_{0}^{\mathrm{d}}\left(T_{j}^{k}\right) \int_{0}^{\tilde{K}^{k}} \Psi_{\gamma}\left(F_{\eta_{j}^{k}}^{T_{j}^{k}} \exp \left(-X_{j}^{k}\right)\right. \\
& =B_{0}^{\mathrm{d}}\left(T_{j}^{k}\right) \int_{0}^{\tilde{K}^{k}} \Psi_{\gamma}\left(F_{-X_{j}^{k}}^{T_{k}^{k}}\left(\log \left(x D_{j}^{k}\right)\right)\right) d x \\
& =B_{0}^{\mathrm{d}}\left(T_{j}^{k}\right) \int_{0}^{\tilde{K}^{k}} \Psi_{\gamma}\left(1-F_{X_{j}^{k}}^{T_{j}^{k}}\left(-\log \left(x D_{j}^{k}\right)\right)\right) d x
\end{aligned}
$$


2. Bid price:

$$
\begin{aligned}
& b_{\gamma}^{T_{j}^{k}}\left(\mathrm{Flt}_{j, K}^{k}\right)=\int_{0}^{\infty}\left(1-\Psi_{\gamma}\left(F_{\mathrm{Flt}_{j, K}^{k}}^{T_{j}^{k}}(x)\right)\right) d x \\
& =\int_{0}^{\infty}\left(1-\Psi_{\gamma}\left(F_{\left(\tilde{K}^{k}-\eta_{j}^{k} \exp \left(-X_{j}^{k}\right)\right)^{+}}^{T_{j}^{k}}\left(x B_{0}^{\mathrm{d}}\left(T_{j}^{k}\right)^{-1}\right)\right)\right) d x \\
& =B_{0}^{\mathrm{d}}\left(T_{j}^{k}\right) \int_{0}^{\infty}\left(1-\Psi_{\gamma}\left(F_{\left(\tilde{K}^{k}-\eta_{j}^{k} \exp \left(-X_{j}^{k}\right)\right)^{+}}^{T^{k}}(x)\right)\right) d x \\
& =B_{0}^{\mathrm{d}}\left(T_{j}^{k}\right) \int_{0}^{\infty}\left(1-\Psi_{\gamma}\left(F_{\left(\tilde{K}^{k}-\eta_{j}^{k} \exp \left(-X_{j}^{k}\right)\right)}^{T_{k}^{k}}(x)\right)\right) d x \\
& =B_{0}^{\mathrm{d}}\left(T_{j}^{k}\right) \int_{0}^{\infty}\left(1-\Psi_{\gamma}\left(1-F_{\eta_{j}^{k} \exp \left(-X_{j}^{k}\right)}^{T_{k}^{k}}\left(\tilde{K}^{k}-x\right)\right)\right) d x \\
& =B_{0}^{\mathrm{d}}\left(T_{j}^{k}\right) \int_{0}^{\tilde{K}^{k}}\left(1-\Psi_{\gamma}\left(1-F_{\eta_{j}^{k} \exp \left(-X_{j}^{k}\right)}^{T_{k}^{k}}\left(\tilde{K}^{k}-x\right)\right)\right) d x \\
& =B_{0}^{\mathrm{d}}\left(T_{j}^{k}\right) \int_{0}^{\tilde{K}^{k}}\left(1-\Psi_{\gamma}\left(1-F_{\eta_{j}^{k} \exp \left(-X_{j}^{k}\right)}^{T_{k}^{k}}(x)\right)\right) d x \\
& =B_{0}^{\mathrm{d}}\left(T_{j}^{k}\right) \int_{0}^{\tilde{K}^{k}}\left(1-\Psi_{\gamma}\left(1-F_{-X_{j}^{k}}^{T_{j}^{k}}\left(\log \left(x D_{j}^{k}\right)\right)\right)\right) d x \\
& =B_{0}^{\mathrm{d}}\left(T_{j}^{k}\right) \int_{0}^{\tilde{K}^{k}}\left(1-\Psi_{\gamma}\left(F_{X_{j}^{k}}^{T_{j}^{k}}\left(-\log \left(x D_{j}^{k}\right)\right)\right)\right) d x .
\end{aligned}
$$

In the one case where we consider the single-curve setting (i.e. $m=0$ ), we obtain:

1. For every $\Psi=\left(\Psi_{\gamma}\right)_{\gamma \geq 0} \in\left\{\Psi^{\mathrm{mv}}, \Psi^{\mathrm{mav}}, \Psi^{\mathrm{mmv}}, \Psi^{\mathrm{mamv}}\right\}$ and $\gamma \geq 0$, it holds

$$
a_{\gamma}^{T_{j-1}}\left(\mathrm{Cpl}_{j, K}^{\mathrm{d}}\right)=B_{0}^{\mathrm{d}}\left(T_{j-1}\right) \tilde{K} \int_{0}^{\tilde{K}^{-1}} \Psi_{\gamma}\left(F_{X_{j}^{\mathrm{d}}}^{T_{j-1}}\left(\log \left(x \eta_{j}^{\mathrm{d}}\right)\right)\right) d x
$$

and

$$
b_{\gamma}^{T_{j-1}}\left(\left.\mathrm{Cp}\right|_{j, K} ^{\mathrm{d}}\right)=B_{0}^{\mathrm{d}}\left(T_{j-1}\right) \tilde{K} \int_{0}^{\tilde{K}^{-1}}\left[1-\Psi_{\gamma}\left(1-F_{X_{j}^{\mathrm{d}}}^{T_{j-1}}\left(\log \left(x \eta_{j}^{\mathrm{d}}\right)\right)\right)\right] d x .
$$

2. For every $\Psi=\left(\Psi_{\gamma}\right)_{\gamma \geq 0} \in\left\{\Psi^{\mathrm{mv}}, \Psi^{\mathrm{mav}}, \Psi^{\mathrm{mmv}}, \Psi^{\text {mamv }}\right\}$ and a permitted $\gamma \geq 0$ for which condition 2.10 is valid, it holds

$$
a_{\gamma}^{T_{j-1}}\left(\mathrm{Flt}_{j, K}^{\mathrm{d}}\right)=B_{0}^{\mathrm{d}}\left(T_{j-1}\right) \tilde{K} \int_{\tilde{K}^{-1}}^{\infty} \Psi_{\gamma}\left(1-F_{X_{j}^{\mathrm{d}}}^{T_{j-1}}\left(\log \left(x \eta_{j}^{\mathrm{d}}\right)\right)\right) d x
$$


and

$$
b_{\gamma}^{T_{j-1}}\left(\mathrm{Flt}{ }_{j, K}^{\mathrm{d}}\right)=B_{0}^{\mathrm{d}}\left(T_{j-1}\right) \tilde{K} \int_{\tilde{K}^{-1}}^{\infty}\left[1-\Psi_{\gamma}\left(F_{X_{j}^{\mathrm{d}}}^{T_{j-1}}\left(\log \left(x \eta_{j}^{\mathrm{d}}\right)\right)\right)\right] d x .
$$

Once again we use the fact that a caplet (floorlet) can be written as a put (call) option on a bond.

On the basis of the analysis we just made above, it follows that we have to identify the cumulative distribution function $F_{X_{j}^{k}}^{T}$ to determine the ask and bid prices. Unfortunately, for a sophisticated driving process $L$ and an advanced volatility function $\Sigma^{k}$ it is, in general, not known. To handle this issue, we proceed as follows:

1. We first determine the characteristic function of $X_{j}^{k}$ under $P_{T}^{\mathrm{d}}$.

2. Then, we apply the common inversion formula stated by Billingsley [13, Theorem 26.2] or Durrett [27] to approximate $F_{X_{j}^{k}}^{T}$. More specifically, as an approximation of the cumulative distribution function, we consider

$$
F_{X_{j}^{k}}^{T}(y) \approx \lim _{M \rightarrow \infty} \frac{1}{\pi} \cdot \int_{0}^{M} \operatorname{Re}\left(\frac{e^{-i u x}-e^{-i u y}}{i u} \varphi_{X_{j}^{k}}^{T}(u)\right) d u
$$

for a suitable $x \in \mathbb{R}$ satisfying $x<y$ and $P_{T}^{\mathrm{d}, X_{j}^{k}}(\{x\})=P_{T}^{\mathrm{d}, X_{j}^{k}}(\{y\})=0$, where $P_{T}^{\mathrm{d}, X_{j}^{k}}$ denotes the distribution of $X_{j}^{k}$ with respect to $P_{T}^{\mathrm{d}}$. The expression 3.53 can be evaluated numerically with some effort.

\section{Ask and Bid Price of Digital Options}

The discounted payoff of a delayed digital option is

$$
\mathrm{DD}_{T}^{k}(w):=B_{0}^{\mathrm{d}}(T) \mathbb{1}_{\left\{w \exp \left(X_{j}^{k}\right)<w \tilde{B}_{k}^{-1} \exp \left(\xi_{j}^{k}\right)\right\}} .
$$

One easily sees that $\operatorname{DD}_{T}^{k}(w)$ is regular with respect to $\alpha^{\mathrm{mv}}, \alpha^{\mathrm{mav}}, \alpha^{\mathrm{mm} v}$ and $\alpha^{\mathrm{mamv}}$. Then, we state its ask and bid price.

Proposition 3.3.4 The ask price of the delayed digital option at level $\gamma \geq 0$ is given by

$$
a_{\gamma}^{T}\left(\operatorname{DD}_{T}^{k}(w)\right)=B_{0}^{\mathrm{d}}(T) \Psi_{\gamma}\left(P_{T}^{\mathrm{d}}\left(w e^{X_{j}^{k}}<w \tilde{B}_{k}^{-1} \exp \left(\xi_{j}^{k}\right)\right)\right)
$$

and the bid price at level $\gamma \geq 0$ can be expressed as

$$
b_{\gamma}^{T}\left(\operatorname{DD}_{T}^{k}(w)\right)=B_{0}^{\mathrm{d}}(T)\left[1-\Psi_{\gamma}\left(P_{T}^{\mathrm{d}}\left(w e^{X_{j}^{k}} \geq w \tilde{B}_{k}^{-1} \exp \left(\xi_{j}^{k}\right)\right)\right)\right] .
$$


Proof: One verifies that the cumulative distribution functions of the random variables $-\mathrm{DD}_{T}^{k}(w)$ and $\mathrm{DD}_{T}^{k}(w)$ under $P_{T}^{\mathrm{d}}$ result in

$$
F_{-\mathrm{DD}_{T}^{k}(w)}^{T}(y)= \begin{cases}1, & y \geq 0 \\ P_{T}^{\mathrm{d}}\left(w \exp \left(X_{j}^{k}\right)<w \tilde{B}_{k}^{-1} \exp \left(\xi_{j}^{k}\right)\right), & y \in\left[-B_{0}^{\mathrm{d}}(T), 0\right) \\ 0, & y<-B_{0}^{\mathrm{d}}(T)\end{cases}
$$

and

$$
F_{\mathrm{DD}_{T}^{k}(w)}^{T}(y)= \begin{cases}1, & y \geq B_{0}^{\mathrm{d}}(T) \\ P_{T}^{\mathrm{d}}\left(w \exp \left(X_{j}^{k}\right) \geq w \tilde{B}_{k}^{-1} \exp \left(\xi_{j}^{k}\right)\right), & y \in\left[0, B_{0}^{\mathrm{d}}(T)\right) \\ 0, & y<0 .\end{cases}
$$

Then, by considering the ask and bid price formulas stated in subsection 2.1.5, we immediately get the statement.

The discounted payoff of a delayed range digital option with barriers $\underline{B}$ and $\bar{B}$ is given by

$$
\mathrm{DRD}_{T}^{k}:=B_{0}^{\mathrm{d}}(T) \cdot \mathbb{1}\left\{\frac{\exp \left(\xi_{j}^{k}\right)}{\overline{\bar{B}}_{k}}<\exp \left(X_{j}^{k}\right)<\frac{\exp \left(\xi_{j}^{k}\right)}{\underline{B}_{k}}\right\} .
$$

We can easily verify that $\mathrm{DRD}_{T}^{k}$ is regular with respect to $\alpha^{\mathrm{mv}}, \alpha^{\mathrm{mav}}, \alpha^{\mathrm{mmv}}$ and $\alpha^{\mathrm{mamv}}$.

Proposition 3.3.5 The ask price of the delayed range digital option at level $\gamma \geq 0$ is given by

$$
a_{\gamma}^{T}\left(\operatorname{DRD}_{T}^{k}\right)=B_{0}^{\mathrm{d}}(T) \Psi_{\gamma}\left(P_{T}^{\mathrm{d}}\left(\frac{\exp \left(\xi_{j}^{k}\right)}{\tilde{\bar{B}}_{k}}<\exp \left(X_{j}^{k}\right)\right)+P_{T}^{\mathrm{d}}\left(\exp \left(X_{j}^{k}\right)<\frac{\exp \left(\xi_{j}^{k}\right)}{\underline{B}_{k}}\right)\right)
$$

and the bid price at level $\gamma \geq 0$ can be expressed as

$$
\begin{aligned}
b_{\gamma}^{T}\left(\operatorname{DRD}_{T}^{k}\right)= & B_{0}^{\mathrm{d}}(T) \\
& \times\left[1-\Psi_{\gamma}\left(P_{T}^{\mathrm{d}}\left(\exp \left(X_{j}^{k}\right) \leq \frac{\exp \left(\xi_{j}^{k}\right)}{\tilde{B}_{k}}\right)+P_{T}^{\mathrm{d}}\left(\frac{\exp \left(\xi_{j}^{k}\right)}{\tilde{B}_{k}} \leq \exp \left(X_{j}^{k}\right)\right)\right)\right] .
\end{aligned}
$$

Proof: Here, the cumulative distribution functions result in

$$
F_{-\mathrm{DRD}_{T}^{k}}^{T}(y)= \begin{cases}1, & y \geq 0 \\ P_{T}^{\mathrm{d}}\left(\frac{\exp \left(\xi_{j}^{k}\right)}{\tilde{B}_{k}}<\exp \left(X_{j}^{k}\right)\right)+P_{T}^{\mathrm{d}}\left(\exp \left(X_{j}^{k}\right)<\frac{\exp \left(\xi_{j}^{k}\right)}{\underline{B}_{k}}\right), & y \in\left[-B_{0}^{\mathrm{d}}(T), 0\right) \\ 0, & y<-B_{0}^{\mathrm{d}}(T)\end{cases}
$$


3.3. Valuation Formulas with Application of the Two-Price Theory

and

$$
F_{\mathrm{DRD}_{T}^{k}}^{T}(y)= \begin{cases}1, & y \geq B_{0}^{\mathrm{d}}(T) \\ P_{T}^{\mathrm{d}}\left(\exp \left(X_{j}^{k}\right) \leq \frac{\exp \left(\xi_{j}^{k}\right)}{\tilde{B}_{k}}\right)+P_{T}^{\mathrm{d}}\left(\frac{\exp \left(\xi_{j}^{k}\right)}{\underline{B}_{k}} \leq \exp \left(X_{j}^{k}\right)\right), & y \in\left[0, B_{0}^{\mathrm{d}}(T)\right) \\ 0, & y<0 .\end{cases}
$$

Then, the claim follows by the formulas of subsection 2.1.5. 



\section{MODEL CALIBRATION}

In this chapter, the two-price multiple-curve model of the previous chapter is calibrated to market data for dates before and during the crisis. More specifically, we consider quoted cap volatilities on $15^{\text {th }}$ August 2006 and on $15^{\text {th }}$ September 2009. The calibration procedure of section 2.4 is applied. Before doing this, we have to convert the cap volatilities in cap prices by using a multiple-curve extension of the standard market model. We also illustrate the calibration results by means of the relative errors of the market and model prices in the appendices.

\subsection{Description and Processing of Cap and Floor Market Data}

The market quotes ask, mid and bid prices of (forward) caps and floors mainly in the form of their implied volatilities (stated annualised and in percentages). This fact involves the consideration of a standard market model. Otherwise it would clearly make no sense to quote prices as a model parameter. The standard market model before the crisis is based on the Black-Scholes-Merton framework (see the seminal papers of Bachelier [5], Osborne [76], Samuelson [80], Black and Scholes [14] and Merton [72]). Generally speaking, this approach considers a world that is forward risk neutral with respect to zero coupon bonds (see Hull [55, chapter 28]).

We closely follow the approach of Mercurio [69] and adjust the classical standard market model to the modern multiple-curve setting. The market model prices of caps (caplets) and floors (floorlets) are then stated. To this end, let $\mathscr{T}^{i}=\left\{T_{0}^{i}, \ldots, T_{n_{i}}^{i}\right\}$ be an arbitrary equidistant discrete tenor structure, where we have $i \in\{0, \ldots, m\}$ and $m \in \mathbb{N}$. As usual, we denote by $\delta^{i}:=\delta^{i}\left(T_{k-1}^{i}, T_{k}^{i}\right)$ the year fraction (tenor) between date $T_{k-1}^{i}$ and $T_{k}^{i}$ for every $k \in\left\{1, \ldots, n_{i}\right\}$. The risk-neutral price of a caplet with strike rate $K$, reset date $T_{k-1}^{i}$ and settlement date $T_{k}^{i}$ for any $k \in\left\{1, \ldots, n_{i}\right\}$ that provides a payoff at 


\section{Model Calibration}

time $T_{k}^{i}$, is given by

$$
\mathrm{Cpl}_{\mathrm{smm}}^{i}\left(T_{k-1}^{i}, T_{k}^{i}, K, \tilde{\sigma}_{k}^{i}\right):=\delta^{i} B_{0}^{\mathrm{d}}\left(T_{k}^{i}\right) \mathbb{E}_{P_{T_{k}^{i}}^{\mathrm{d}}}\left[\left(L_{T_{k-1}^{i}}^{i}\left(T_{k-1}^{i}, T_{k}^{i}\right)-K\right)^{+}\right] .
$$

We emphasise that we obtain the classical single-curve market formula by setting $i=0$ and using the fact that $L_{T_{k-1}}^{\mathrm{d}}\left(T_{k-1}, T_{k}\right)$ is assumed to be log-normally distributed and $L^{\mathrm{d}}\left(T_{k-1}, T_{k}\right)$ is a martingale under $P_{T_{k}}^{\mathrm{d}}$. Unfortunately, for every $i \in\{1, \ldots, m\}$, the forward reference rate $L^{i}\left(T_{k-1}^{i}, T_{k}^{i}\right)$ is not a $P_{T_{k}^{i}}^{\mathrm{d}}$-martingale in general.

To solve this problem, we replace the forward rate with its conditional expected value (the time- $t$ (modified) FRA-rate; cf. subsection 2.2.4 and the relations 2.15) and (2.16), that is a martingale under the underlying pricing measure. More specifically, we consider

$$
K_{t, k}^{i}=\mathbb{E}_{P_{T_{k}^{i}}^{\mathrm{d}}}\left[L_{T_{k-1}^{i}}^{i}\left(T_{k-1}^{i}, T_{k}^{i}\right) \mid \mathscr{G}_{t}\right]
$$

as the key quantity to model (by definition, it is a $P_{T_{k}^{i}}^{\mathrm{d}}$-martingale). Note that we have

$$
K_{T_{k-1}^{i}, k}^{i}=L_{T_{k-1}^{i}}^{i}\left(T_{k-1}^{i}, T_{k}^{i}\right) .
$$

Therefore, the caplet pricing formula can be written as

$$
\mathrm{Cpl}_{\mathrm{smm}}^{i}\left(T_{k-1}^{i}, T_{k}^{i}, K, \sigma_{k}^{i}\right)=\delta^{i} B_{0}^{\mathrm{d}}\left(T_{k}^{i}\right) \mathbb{E}_{P_{T_{k}^{i}}^{\mathrm{d}}}\left[\left(K_{T_{k-1}^{i}, k}^{i}-K\right)^{+}\right] .
$$

The dynamics $K^{i}$ are assumed to be given by

$$
K_{t, k}^{i}=K_{0, k}^{i} \exp \left(\sigma_{k}^{i} W_{t}-\frac{1}{2}\left(\sigma_{k}^{i}\right)^{2} t\right), \quad \text { for any } t \in\left[0, T_{k-1}^{i}\right],
$$

where $W=\left(W_{t}\right)_{t \in\left[0, T_{k-1}^{i}\right]}$ is a standard Wiener process under $P_{T_{k}^{i}}^{\mathrm{d}}, \sigma_{k}^{i}>0$ is a constant volatility and $K_{0, k}^{i}$ is approximately given by

$$
K_{0, k}^{i} \approx L_{0}^{i}\left(T_{k-1}^{i}, T_{k}^{i}\right)=\frac{1}{\delta}\left(\frac{B_{0}^{i}\left(T_{k-1}^{i}\right)}{B_{0}^{i}\left(T_{k}^{i}\right)}-1\right) .
$$

Note that the initial curves $B_{0}^{\mathrm{d}}$ and $B_{0}^{i}$ are constructed by using the bootstrapping method developed by Ametrano and Bianchetti [3]. Since $K_{T_{k-1}^{i}, k}^{i}$ is log-normally distributed under $P_{T_{k}^{i}}^{\mathrm{d}}$, we conclude that the multiple-curve market model price 4.1 becomes

$$
\mathrm{Cpl}_{\mathrm{smm}}^{i}\left(T_{k-1}^{i}, T_{k}^{i}, K, \sigma_{k}^{i}\right)=\delta^{i} B_{0}^{\mathrm{d}}\left(T_{k}^{i}\right) \cdot\left(K_{0, k}^{i} \cdot \Phi\left(d_{1, k}^{i}\right)-K \cdot \Phi\left(d_{2, k}^{i}\right)\right) \text {, }
$$


where $\Phi$ denotes the standard Gaussian cumulative distribution function,

$$
d_{1, k}^{i}:=\frac{\ln \left(\frac{K_{0, k}^{i}}{K}\right)+\frac{1}{2}\left(\sigma_{k}^{i}\right)^{2} T_{k-1}^{i}}{\sigma_{k}^{i} \sqrt{T_{k-1}^{i}}}
$$

and

$$
d_{2, k}^{i}:=d_{1, k}^{i}-\sigma_{k}^{i} \sqrt{T_{k-1}^{i}}=\frac{\ln \left(\frac{K_{0, k}^{i}}{K}\right)-\frac{1}{2}\left(\sigma_{k}^{i}\right)^{2} T_{k-1}^{i}}{\sigma_{k}^{i} \sqrt{T_{k-1}^{i}}} .
$$

Analogously, the model price of the corresponding floorlet can be expressed as

$$
\mathrm{Flt}_{\mathrm{smm}}^{i}\left(T_{k-1}^{i}, T_{k}^{i}, K, \sigma_{k}^{i}\right):=\delta^{i} B_{0}^{\mathrm{d}}\left(T_{k}^{i}\right) \cdot\left(K \cdot \Phi\left(-d_{2, k}^{i}\right)-K_{0, k}^{i} \cdot \Phi\left(-d_{1, k}^{i}\right)\right) .
$$

To sum up, the modern multiple-curve market model prices of caplets and floorlets are again stated in a Black-Scholes-Merton framework. The differences with respect to the classical formulas are given by the underlying rate, which is now the FRA-rate, and by the discount factors that are extracted from the discount curve $d$.

The market formula for the price of the interest rate cap that is settled in arrears and matures at $T_{n_{i}}^{i}$ with fixing and payment dates along $\mathscr{T}^{i}$ is given by

$$
\operatorname{Cap}_{0}^{\mathrm{smm}}\left(\mathscr{T}^{i}, K, \sigma_{T_{n_{i}}^{i}}^{i}\right):=\sum_{k=1}^{n_{i}} \mathrm{Cpl}_{\mathrm{smm}}^{i}\left(T_{k-1}^{i}, T_{k}^{i}, K, \sigma_{T_{n_{i}}^{i}}^{i}\right) .
$$

The corresponding formula of the floor is

$$
\text { Floor }_{0}^{\mathrm{smm}}\left(\mathscr{T}^{i}, K, \sigma_{T_{n_{i}}^{i}}^{i}\right):=\sum_{k=1}^{n_{i}} \mathrm{Flt}_{\mathrm{smm}}^{i}\left(T_{k-1}^{i}, T_{k}^{i}, K, \sigma_{T_{n_{i}}^{i}}^{i}\right) .
$$

Observe that the same volatility $\sigma_{T_{n_{i}}^{i}}^{i}$ is used for all caplets (floorlets) of the cap (floor). This volatility is called flat or forward volatility. It is exactly the quoted implied volatility of caps (floors) in the market. An alternative way is to use a different volatility for each caplet (floorlet). These types of volatilities are referred to as spot or forward forward volatilities.

The spot volatilities can be calculated from flat volatilities and vice versa under the consistency conditions

$$
\left.\sum_{k=1}^{n_{i}} \mathrm{Cp}\right|_{\mathrm{smm}} ^{i}\left(T_{k-1}^{i}, T_{k}^{i}, K, \sigma_{T_{n_{i}}^{i}}^{i}\right)=\left.\sum_{k=1}^{n_{i}} \mathrm{Cp}\right|_{\mathrm{smm}} ^{i}\left(T_{k-1}^{i}, T_{k}^{i}, K, \sigma_{k}^{i}\right)
$$




\section{Model Calibration}

and

$$
\sum_{k=1}^{n_{i}} \mathrm{Flt}_{\mathrm{smm}}^{i}\left(T_{k-1}^{i}, T_{k}^{i}, K, \sigma_{T_{n_{i}}^{i}}^{i}\right)=\sum_{k=1}^{n_{i}} \mathrm{Flt}_{\mathrm{smm}}^{i}\left(T_{k-1}^{i}, T_{k}^{i}, K, \sigma_{k}^{i}\right) .
$$

We emphasise that we use spot volatilities on the right-hand side of these equations. Without these conditions there is a kind of inconsistency in cap volatilities: The same caplet could be linked to different (spot) volatilities when it is related to different caps with separate flat volatility.

We briefly describe the bootstrapping technique to extract spot volatilities from the flat volatilities and vice versa:

1. The computations of the flat volatilities from the spot volatilities are evident.

2. The spot volatilities $\sigma_{1}^{i}, \ldots, \sigma_{n_{i}}^{i}$ are inductively determined by the relations

$$
\operatorname{Cap}_{0}^{\mathrm{smm}}\left(\left\{T_{0}^{i}, \ldots, T_{m}^{i}\right\}, K, \sigma_{T_{m}^{i}}^{i}\right)=\sum_{k=1}^{m} \mathrm{Cpl}_{\mathrm{smm}}^{i}\left(T_{k-1}^{i}, T_{k}^{i}, K, \sigma_{k}^{i}\right)
$$

or

$$
\text { Floor }_{0}^{\mathrm{smm}}\left(\left\{T_{0}^{i}, \ldots, T_{m}^{i}\right\}, K, \sigma_{T_{m}^{i}}^{i}\right)=\sum_{k=1}^{m} \mathrm{Flt}_{\mathrm{smm}}^{i}\left(T_{k-1}^{i}, T_{k}^{i}, K, \sigma_{k}^{i}\right)
$$

where we start with $m=1$ and proceed up to $m=n_{i}$. In each step, we compute the spot volatility as (unique) root of a function. They are calculated by applying the method developed by Soetaert and Herman [85, examples from chapter 7] and by using the R-package 'rootSolve' (see Soetaert [91]).

Note that we can compute a caplet (floorlet) price by subtracting two consecutive cap (floor) prices. For instance, the price of a caplet related to the six-month curve and a maturity of three years is given by the difference between the three-year and the 2.5-year cap price.

The quoted implied volatilities for caps and floors typically have maturities of $1,2, \ldots, 20$ years and strike rates of $1.75,2.00,2.25,2.50,3.00,3.50,4.00,5.00, \ldots, 9.00,10.00$ percent. We focus on most liquid caps and floors with maturities $1,2, \ldots, 10$ in the European market. Therefore, the reference rate is the Euribor. It is market convention that quotes for caps with maturities up to two years are indexed on the three-month Euribor and quotes with maturities greater than two years are indexed on the six-month Euribor. The missing implied volatilities are obtained by cubic spline interpolation between the quoted maturities and extrapolation. We use the method developed by Forsythe, Moler, and Malcolm [47] for the interpolation. This method is implemented in the R-package 
'Stats' created by the R Core Team [92]. The extrapolation is made with the help of the R-package 'Hmisc' created by Harrell Jr [88].

We stress that we can use the single-curve approach for data sets before the crisis. But when we consider data sets during the crisis, we have to apply our multiple-curve model.

\subsection{Calibration of Caps in the Two-Price and Single-Curve Setting}

To illustrate the calibration procedure, we first consider the data set before the crisis. The two-price theory and the single-curve approach are applied.

\subsubsection{Data Sets}

Figure 4.1 presents a surface of the quoted implied mid volatilities for caps on $15^{\text {th }}$ August 2006. We extend the values to the missing maturities as described in the previous section. The initial zero coupon bond prices are listed in table 4.1 and 4.2 . The market model prices of caps can be derived from valuation formula 4.2 . Then, we apply the bootstrapping method of section 4.1 to extract the spot volatilities of the caplets. The resulting spot volatilities are illustrated in figure 4.2. The ask and bid spreads between the implied (spot) volatilities of caplets for some special maturities and strike rates are given in figure 4.3 and 4.4

\begin{tabular}{ll}
\hline Maturity & Initial Bond Price \\
\hline 0.25 Year & 0.992560 \\
0.50 Year & 0.984083 \\
0.75 Year & 0.975181 \\
1.00 Year & 0.966156 \\
1.25 Year & 0.957169 \\
1.50 Year & 0.948271 \\
1.75 Year & 0.939486 \\
2.00 Year & 0.930791 \\
\hline
\end{tabular}

Table 4.1.: Discount bond prices $B_{0}^{\mathrm{d}}(T)$ on $15^{\text {th }}$ August 2006 .

\subsubsection{Calibration Procedure}

We proceed as described in section 2.4. Specifically, we consider

$$
\begin{gathered}
H=\{(T, K): T \in\{0.5,0.75,1,1.25,1.5,1.75,2,2.5,3,3.5, \ldots, 9.5,10\}, \\
K \in\{0.0175,0.02,0.0225,0.025,0.03,0.04, \ldots, 0.1\}\}
\end{gathered}
$$




\section{Cap volatility surface}

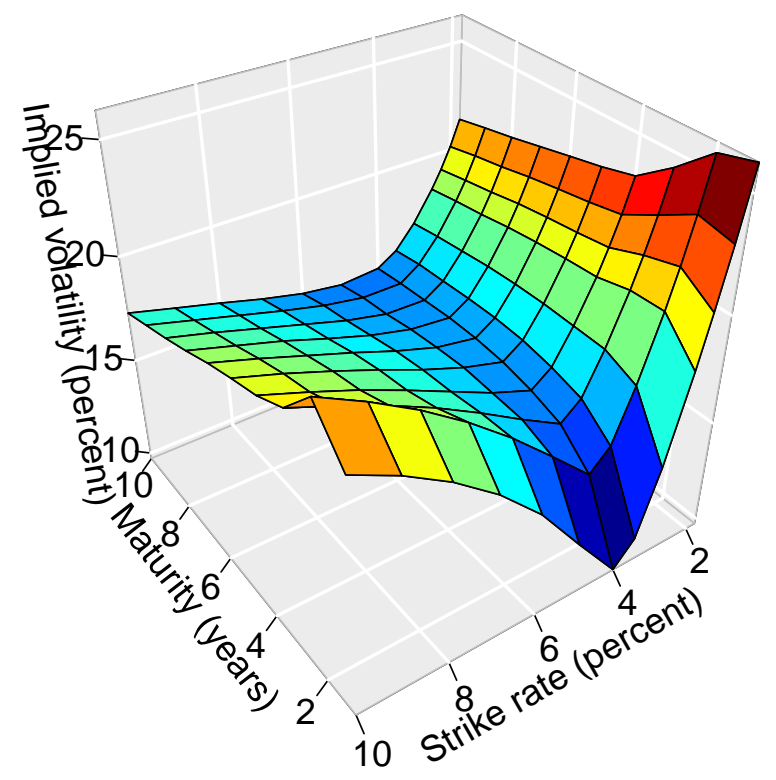

Figure 4.1.: EUR cap market implied volatility surface on $15^{\text {th }}$ August 2006.

and

$$
\bar{H}_{T}=\{(T, K): K \in\{0.0175,0.02,0.0225,0.025,0.03\}\} \subset H
$$

for any $T \in\{1.25,1.5,1.75,2\}$. Therefore, we have $T^{*}=10$. The corresponding ask, mid and bid market prices of caplets are denoted by $\Pi^{\text {ask }}(T, K), \Pi^{\text {mid }}(T, K)$ and $\Pi^{\text {bid }}(T, K)$. Their model prices are stated in 3.51), 3.46) and (3.52). Obviously, it holds

$$
\mathrm{Cpl}_{0}\left(T_{j-1}, T_{j}, K\right)=a_{0}^{T_{j-1}}\left(\mathrm{Cpl}_{j, K}^{\mathrm{d}}\right)=b_{0}^{T_{j-1}}\left(\mathrm{Cpl}_{j, K}^{\mathrm{d}}\right) .
$$

Since the model prices depend on parameters, we set

$$
\begin{aligned}
a\left(T_{j-1}, K, \theta, \gamma\right) & :=a_{\gamma}^{T_{j-1}}\left(\mathrm{Cpl}_{j, K}^{\mathrm{d}}\right) \\
\mathrm{Cpl}_{0}\left(T_{j-1}, K, \theta\right) & :=\mathrm{Cpl}_{0}\left(T_{j-1}, T_{j}, K\right) \\
b\left(T_{j-1}, K, \theta, \gamma\right) & :=b_{\gamma}^{T_{j-1}}\left(\mathrm{Cpl}_{j, K}^{\mathrm{d}}\right),
\end{aligned}
$$

where we denote by $\Theta$ the set of admissible model parameters and $\theta \in \Theta$. 


\begin{tabular}{ll}
\hline Maturity & Initial Bond Price \\
\hline 0.50 Year & 0.984083 \\
1.00 Year & 0.966156 \\
1.50 Year & 0.948271 \\
2.00 Year & 0.930791 \\
2.50 Year & 0.913680 \\
3.00 Year & 0.896793 \\
3.50 Year & 0.880104 \\
4.00 Year & 0.863501 \\
4.50 Year & 0.846966 \\
5.00 Year & 0.830523 \\
5.50 Year & 0.814167 \\
6.00 Year & 0.797894 \\
6.50 Year & 0.781750 \\
7.00 Year & 0.765742 \\
7.50 Year & 0.749930 \\
8.00 Year & 0.734269 \\
8.50 Year & 0.718884 \\
9.00 Year & 0.703667 \\
9.50 Year & 0.688686 \\
10.0 Year & 0.673950 \\
\hline
\end{tabular}

Table 4.2.: Discount bond prices $B_{0}^{\mathrm{d}}(T)$ on $15^{\text {th }}$ August 2006.

In the first step of the calibration procedure, we solve the minimisation problem:

$$
O_{1}(\hat{\theta}, H)=\min _{\theta \in \Theta} \sum_{T, K \in H}\left(\mathrm{Cpl}_{0}(T, K, \theta)-\Pi^{\mathrm{mid}}(T, K)\right)^{2} .
$$

We obtain an admissible $\hat{\theta}$ by using the methods stated in section 2.4. At this stage, this situation corresponds to the classical no-arbitrage valuation theory with one price (cf. Delbaen and Schachermayer [25]). Then, we proceed with the second step. Recall that the (il)liquidity parameter depends on the maturity of the caplet and we therefore consider the parameter $\gamma_{j}$, where we have $j \in\{1,2,3,4\}$. Consequently, for every $j \in\{1,2,3,4\}$, we continue to determine the parameter $\hat{\gamma}_{j}$ by solving

$$
O_{2}\left(\hat{\gamma}_{j}, \hat{\theta}, \bar{H}_{T_{j}}\right)=\min _{\gamma_{j} \geq 0} O_{2}\left(\gamma_{j}, \hat{\theta}, \bar{H}_{T_{j}}\right)
$$




\section{Caplet implied volatility surface}

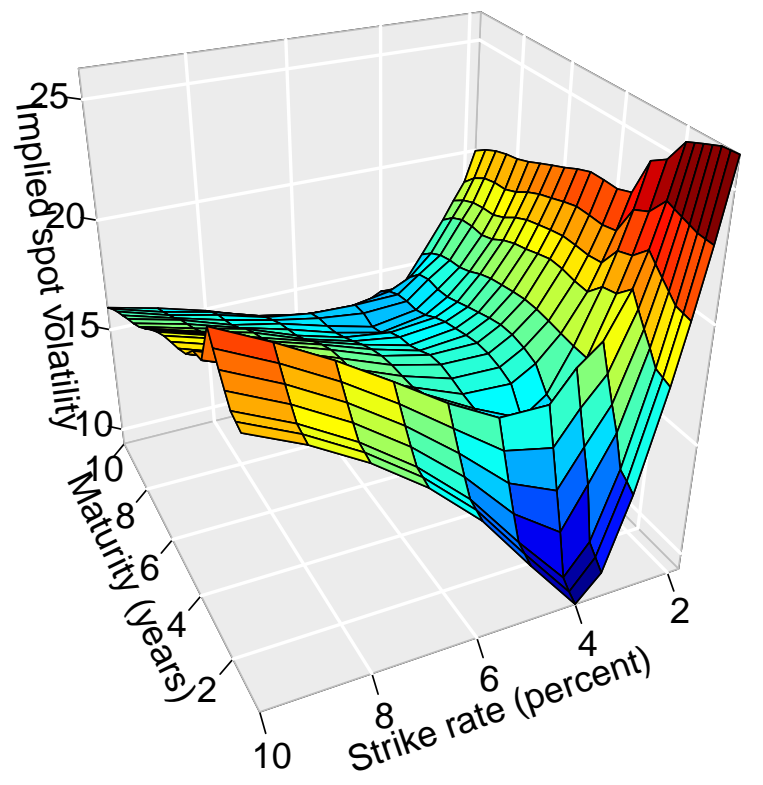

Figure 4.2.: Extended EUR caplet implied spot volatility surface on $15^{\text {th }}$ August 2006.

where the objective function is given by

$$
\begin{aligned}
& O_{2}\left(\gamma_{j}, \hat{\theta}, \bar{H}_{T_{j}}\right)=\sum_{\left(T_{j}, K\right) \in \bar{H}_{T_{j}}}[\left(a\left(T_{j-1}, K, \hat{\theta}, \gamma_{j}\right)-\Pi^{\mathrm{ask}}\left(T_{j-1}, K\right)\right)^{2} \\
&\left.+\left(b\left(T_{j-1}, K, \hat{\theta}, \gamma_{j}\right)-\Pi^{\mathrm{bid}}\left(T_{j-1}, K\right)\right)^{2}\right]
\end{aligned}
$$

and $T_{j} \in\{1.25,1.5,1.75,2\}$.

\subsubsection{Model Framework (I)}

The variance gamma process is studied by Madan and Seneta [67] and Madan and Milne [66]. Another interesting application of this process is given by Madan, Carr, and Chang [68]. For practical purposes, we refer to Seneta [83] and the R-package 'VarianceGamma' created by Scott [90]. Note that the variance gamma distribution can be obtained as limiting case of the generalised hyperbolic distribution (see Eberlein and Hammerstein [31]). Thus, we are able to characterise the set of distribution parameters in two ways: 


\section{Caplet volatility spread}

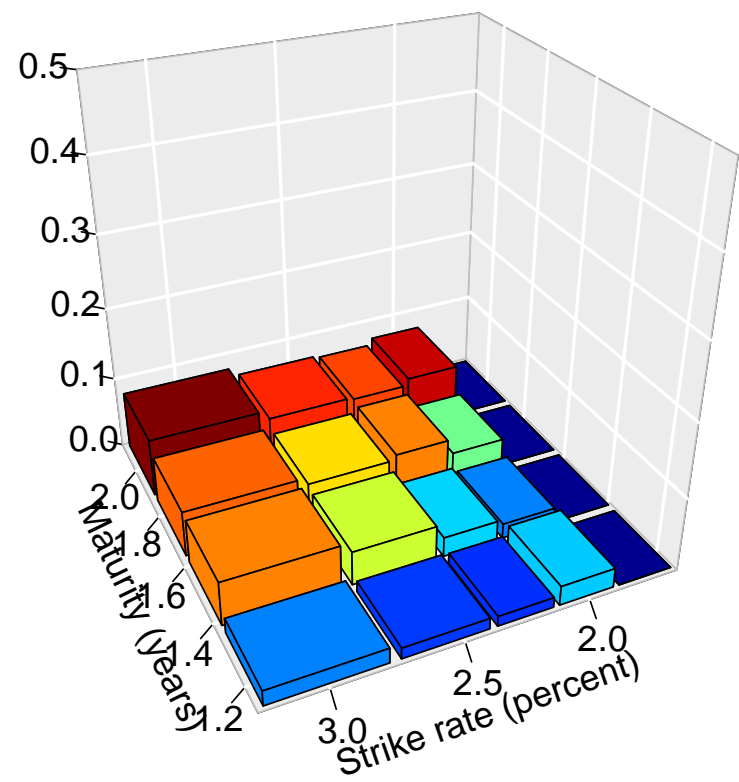

Figure 4.3.: Spread between ask and bid (spot) volatilities of caplets on $15^{\text {th }}$ August 2006. We consider maturities of $1.25,1.5,1.75$ and 2 years and strike rates of $1.75,2,2.25,2.5,3$ percent.

(i) $(\sigma, \nu, \theta, c) \in \Lambda_{1}:=(0, \infty) \times(0, \infty) \times \mathbb{R} \times \mathbb{R}$.

(ii) $(\lambda, \alpha, \beta, \mu) \in \Lambda_{2}:=\left\{\left(\lambda_{0}, \alpha_{0}, \beta_{0}, \mu_{0}\right) \in \Lambda_{1}|| \beta_{0} \mid<\alpha_{0}\right\}$.

The relations between both parameter representations are given by

$$
\lambda=\frac{1}{\nu} \quad \alpha=\sqrt{\frac{2}{\nu \sigma^{2}}+\left(\frac{\theta}{\sigma^{2}}\right)^{2}} \quad \beta=\frac{\theta}{\sigma^{2}} \quad \mu=c
$$

and

$$
\nu=\frac{1}{\lambda} \quad \sigma^{2}=\frac{2 \lambda}{\alpha^{2}-\beta^{2}} \quad \theta=\frac{2 \lambda \beta}{\alpha^{2}-\beta^{2}} \quad c=\mu .
$$

We consider the following volatility structure and driving process $(d=1)$ : 


\section{Caplet volatility spread}

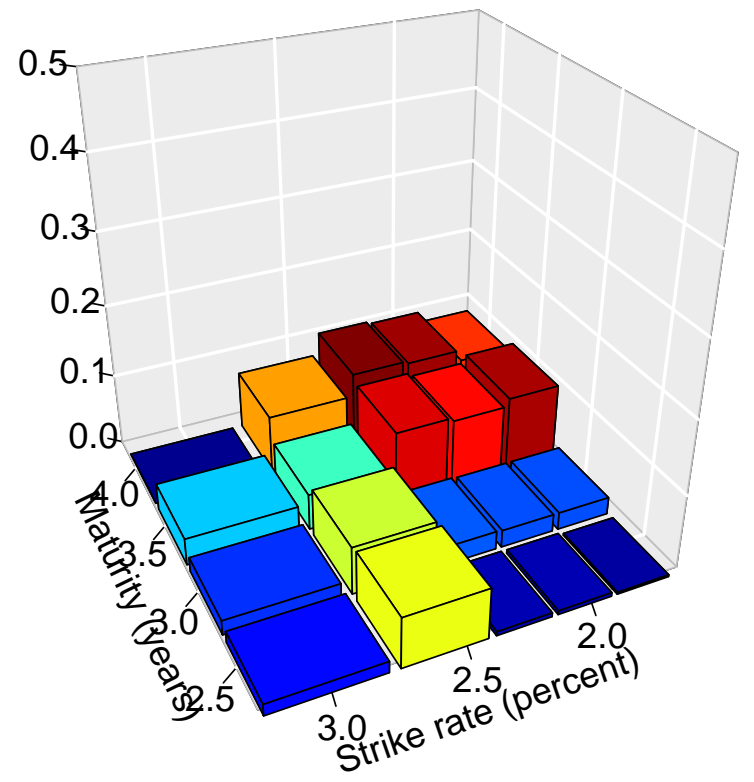

Figure 4.4.: Spread between ask and bid (spot) volatilities of caplets on $15^{\text {th }}$ August 2006. We consider maturities of 2.5,3,3.5 and 4 years and strike rates of $1.75,2,2.25,2.5,3$ percent.

(Vol.I) Vasicek volatility structure

$$
\sigma^{\mathrm{d}}(t, T)=\hat{\sigma} \exp (-a(T-t))
$$

with

$$
\Sigma^{\mathrm{d}}(t, T)= \begin{cases}\frac{\hat{\sigma}}{a}(1-\exp (-a(T-t))), & \text { when } t \leq T \\ 0, & \text { when } t>T .\end{cases}
$$

(DP.I) Variance-Gamma process $L=\left(L_{t}\right)_{t \in\left[0, T^{*}\right]}$ defined on $\hat{\mathscr{B}}$.

The generating distribution of the Variance-Gamma process possesses a moment generating function of the form

$$
M_{\mathrm{VG}(\lambda, \alpha, \beta, \mu)}(u)=e^{\mu u}\left(\frac{\alpha^{2}-\beta^{2}}{\alpha^{2}-(\beta+u)^{2}}\right)^{\lambda}
$$


for every $u \in(-\alpha-\beta, \alpha-\beta)$. Equivalently, we obtain

$$
M_{\mathrm{VG}(\sigma, \nu, \theta, c)}(u)=e^{c u}\left(\frac{1}{1-\nu \theta u-\frac{\sigma^{2} \nu}{2} u^{2}}\right)^{1 / \nu} .
$$

The characteristic function results in

$$
\varphi_{\mathrm{VG}(\lambda, \alpha, \beta, \mu)}(u)=e^{i \mu u}\left(\frac{\alpha^{2}-\beta^{2}}{\alpha^{2}-(\beta+i u)^{2}}\right)^{\lambda}
$$

where we exploit the relation $\varphi_{\mathrm{VG}(\lambda, \alpha, \beta, \mu)}(u)=M_{\mathrm{VG}(\lambda, \alpha, \beta, \mu)}(i u)$. Analogously, we get

$$
\varphi_{\mathrm{VG}(\sigma, \nu, \theta, c)}(u)=e^{i c u}\left(\frac{1}{1-i \nu \theta u+\frac{\sigma^{2} \nu}{2} u^{2}}\right)^{1 / \nu} .
$$

Then, there is an analytic extension of the characteristic function to the strip $\mathbb{R}-i(-\alpha-$ $\beta, \alpha-\beta) \subset \mathbb{C}$ (cf. Sato [81, Theorem 25.17]). From the simple form of the characteristic function, we deduce that

$$
L_{t} \sim \mathrm{VG}(\sqrt{t} \sigma, \nu / t, t \theta, t c)
$$

for every $t \in\left(0, T^{*}\right]$.

It is more comfortable to work with parameter set $(i i)$ due to the form of the characteristic function. Let us define $m(\alpha, \beta):=\min \{|-\alpha-\beta|, \alpha-\beta\}$ and fix a $\rho$ with $0<\rho<$ $m(\alpha, \beta)$. We choose $\epsilon$ and $M$ from condition $(\mathbb{E M})$ such that $(1+\epsilon) M=m(\alpha, \beta)-\rho$. Then, we specify $\hat{M}$ in such a way that it satisfies $0<\hat{M}<\min \{1, M\}$. Since the parameter $\hat{\sigma}$ of the volatility structure can be eliminated without loss of generality (cf. Eberlein and Kluge [33]), we choose $\hat{\sigma}=|a|$. The justification for this approach is that a multiplicative constant of the volatility structure can be included in the distribution parameters of $L$ and it is therefore redundant. Then, it follows that

$$
\Sigma^{\mathrm{d}}(t, T)= \begin{cases}\operatorname{sign}(a)(1-\exp (-a(T-t))), & \text { when } t \leq T \\ 0, & \text { when } t>T\end{cases}
$$

Notice that, for every $a \neq 0$, it holds that $\Sigma^{\mathrm{d}}(t, T) \geq 0$ for all $(t, T) \in\left[0, T^{*}\right] \times\left[0, T^{*}\right]$. Further, we only consider parameters $a \neq 0$ which satisfy

$$
\sup _{0 \leq t \leq T \leq T^{*}}\{\operatorname{sign}(a)(1-\exp (-a(T-t)))\} \leq \hat{M}<M .
$$

It follows that any parameter $a$ has to be restricted to the set $\left[-\frac{\ln (1+\hat{M})}{10},-\frac{\ln (1-\hat{M})}{10}\right] \backslash\{0\}$. Moreover, the mapping $\left[0, T^{*}\right] \ni t \mapsto \hat{\sigma} \exp (-a(T-t)) \in \mathbb{R}$ is obviously continuous for 


\section{Model Calibration}

every $T \in\left[0, T^{*}\right]$. Consequently, condition $(\mathbb{D E} \mathbb{T})$ is satisfied. As seen in section 3.3 .1 we have to choose $R_{1} \in\left[-\frac{M-\hat{M}}{\hat{M}}, 0\right)$ in the caplet valuation formula. The extended cumulant function $\theta_{s}$ of $L$ results in

$$
\theta_{s}(z)=\mu z+\lambda \ln \left(\frac{\alpha^{2}-\beta^{2}}{\alpha^{2}-(\beta+z)^{2}}\right)
$$

for every $z \in \mathbb{C}$ with $|\operatorname{Re}(z)| \leq M$ (cf. Sato [81, Theorem 25.17]). Then, by using expression 3.40 , the characteristic function of $X_{j}^{\mathrm{d}}$ under $P_{T_{j-1}}^{\mathrm{d}}$ at point $u-i R_{1}$ is

$$
\begin{aligned}
& \varphi_{X_{j}^{\mathrm{d}}}^{T_{j-1}}\left(u-i R_{1}\right) \\
= & \exp \left(\int_{0}^{T_{j-1}}-\mu \operatorname{sign}(a)\left(R_{1}+i u\right)\left[\exp \left(-a\left(T_{j}-s\right)\right)-\exp \left(-a\left(T_{j-1}-s\right)\right)\right]\right. \\
& \left.+\lambda \ln \left(\frac{\alpha^{2}-\left(\beta+\operatorname{sign}(a)\left(1-\exp \left(-a\left(T_{j-1}-s\right)\right)\right)\right)^{2}}{\alpha^{2}-\left(\beta+\eta_{j}\left(s, u, a, R_{1}\right)\right)^{2}}\right) d s\right),
\end{aligned}
$$

where we set

$$
\begin{aligned}
\eta_{j}\left(s, u, a, R_{1}\right):= & \operatorname{sign}(a)\left(1-\exp \left(-a\left(T_{j-1}-s\right)\right)\right. \\
& \left.+\left(R_{1}+i u\right)\left[\exp \left(-a\left(T_{j-1}-s\right)\right)-\exp \left(-a\left(T_{j}-s\right)\right)\right]\right) .
\end{aligned}
$$

The deterministic part $D_{j}^{\mathrm{d}}$ can easily be calculated as

$$
\begin{aligned}
D_{j}^{\mathrm{d}}=\frac{B_{0}^{\mathrm{d}}\left(T_{j}\right)}{B_{0}^{\mathrm{d}}\left(T_{j-1}\right)} \exp ( & \int_{0}^{T_{j-1}} \mu \operatorname{sign}(a)\left[\exp \left(-a\left(T_{j}-s\right)\right)-\exp \left(-a\left(T_{j-1}-s\right)\right)\right] \\
& \left.+\lambda \ln \left(\frac{\alpha^{2}-\left(\beta+\operatorname{sign}(a)\left(1-\exp \left(-a\left(T_{j}-s\right)\right)\right)\right)^{2}}{\alpha^{2}-\left(\beta+\operatorname{sign}(a)\left(1-\exp \left(-a\left(T_{j-1}-s\right)\right)\right)^{2}\right.}\right) d s\right)
\end{aligned}
$$

and we obtain $\xi_{j}=-\ln D_{j}^{\mathrm{d}}$ and $\eta_{j}^{\mathrm{d}}=\frac{1}{D_{j}^{\mathrm{d}}}$. The caplet (mid) model price can be determined by

$$
\mathrm{Cpl}_{0}\left(T_{j-1}, T_{j}, K\right)=\frac{B_{0}^{\mathrm{d}}\left(T_{j-1}\right) \tilde{K}}{\pi} \cdot I_{j}\left(\lambda, \alpha, \beta, a, R_{1}, K\right)
$$


where we define

$$
\begin{aligned}
& I_{j}\left(\lambda, \alpha, \beta, a, R_{1}, K\right):=\int_{0}^{\infty} \operatorname{Re}\left(\left[\frac{B_{0}^{\mathrm{d}}\left(T_{j}\right)}{B_{0}^{\mathrm{d}}\left(T_{j-1}\right)}\right]^{R_{1}+i u}\right. \\
& \times \frac{\tilde{K}^{-\left(1-R_{1}-i u\right)}}{\left(-R_{1}-i u\right)\left(1-R_{1}-i u\right)} \\
& \times \exp \left(\int_{0}^{T_{j-1}} \lambda \ln \left(\frac{\alpha^{2}-\left(\beta+\operatorname{sign}(a)\left(1-\exp \left(-a\left(T_{j-1}-s\right)\right)\right)\right)^{2}}{\alpha^{2}-\left(\beta+\eta_{j}\left(s, u, a, R_{1}\right)\right)^{2}}\right)\right. \\
& \left.\left.+\left(R_{1}+i u\right) \lambda \ln \left(\frac{\alpha^{2}-\left(\beta+\operatorname{sign}(a)\left(1-\exp \left(-a\left(T_{j}-s\right)\right)\right)\right)^{2}}{\alpha^{2}-\left(\beta+\operatorname{sign}(a)\left(1-\exp \left(-a\left(T_{j-1}-s\right)\right)\right)^{2}\right.}\right) d s\right)\right) d u
\end{aligned}
$$

We observe that the parameter $\mu$ (respectively $c$ ) does not appear in valuation formula 4.3. Therefore, this parameter does not have an impact on the price and $\mu$ (respectively c) can be set equal to zero (cf. Eberlein and Kluge [33]).

Finally, the ask and bid model prices of the caplet are obtained by valuation formulas (3.51) and 3.52.

\subsubsection{Calibration Results of Model Framework (I)}

We present the calibration results of model framework (I). The calibrated distribution parameters are illustrated in table 4.3 and the calibrated volatility structure parameter is given in table 4.4 .

\begin{tabular}{llll}
\hline Parameter Type (i) & Value & Parameter Type (ii) & Value \\
\hline$\sigma$ & 0.281652 & $\lambda$ & 0.580 \\
$\nu$ & 1.724138 & $\alpha$ & 3.870 \\
$\theta$ & -0.047200 & $\beta$ & -0.595 \\
\hline
\end{tabular}

Table 4.3.: Calibrated parameters of variance gamma distribution.

\begin{tabular}{ll}
\hline Parameter & Value \\
\hline$a$ & 0.024 \\
\hline
\end{tabular}

Table 4.4.: Calibrated parameter of Vasicek volatility structure.

The value of the objective function results in

$$
O_{1}(\hat{\theta}, H)=2.956576 \cdot 10^{-06} \text {. }
$$

The market prices of caplets and caps are excellently fitted for strike rates up to six 


\section{Model Calibration}

percent as we can see in figures 4.5 and 4.6 . We also illustrate the calibration results in form of the relative errors between market and model prices, i.e. we consider

$$
\text { relative error }=\left|\frac{\text { model price }- \text { market price }}{\text { market price }}\right| \text {. }
$$

They are presented in figures A.1 and A.2 in the appendix. We stress that the discrepancies for short maturities and strike rates greater than six percent result from the very small values of the related prices.
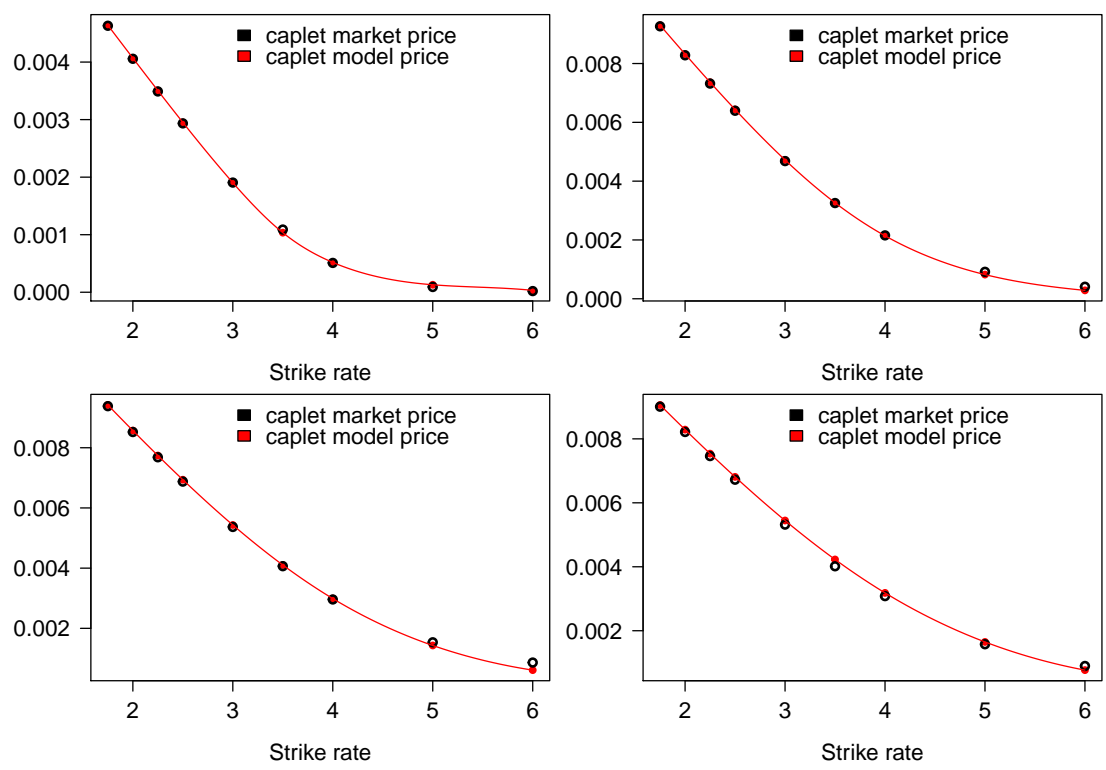

Figure 4.5.: Market and model (mid) prices of caplets with maturity of two years (top left), five years (top right), eight years (bottom left) and ten years (bottom right) for strike rates of $1.75,2.00,2.25,2.50,3.00,3.50,4.00,5.00$ and 6.00 percent on $15^{\text {th }}$ August 2006.

Figure 4.7 shows the fit of the implied volatilities of the cap for a special maturity. It is a well-known fact that an homogeneous Lévy process is not able to fit precisely the implied volatilities along all maturities. To achieve this, we have to use time-inhomogeneous Lévy processes (see Eberlein and Kluge [34]).

The calibrated parameters $\gamma$ with the corresponding values of the objective function $\mathrm{O}_{2}$ are listed in table 4.5. We use the family $\left(\Psi_{\gamma}^{\mathrm{mmv}}\right)_{\gamma \geq 0}$ of MINMAXVAR distortion functions for the calibration.

We visualise the nature of the underlying driving process by means of the density function of the calibrated variance gamma distribution in figure 4.8 and four sample paths in figure 4.9. The R-package 'VarianceGamma' is applied. 

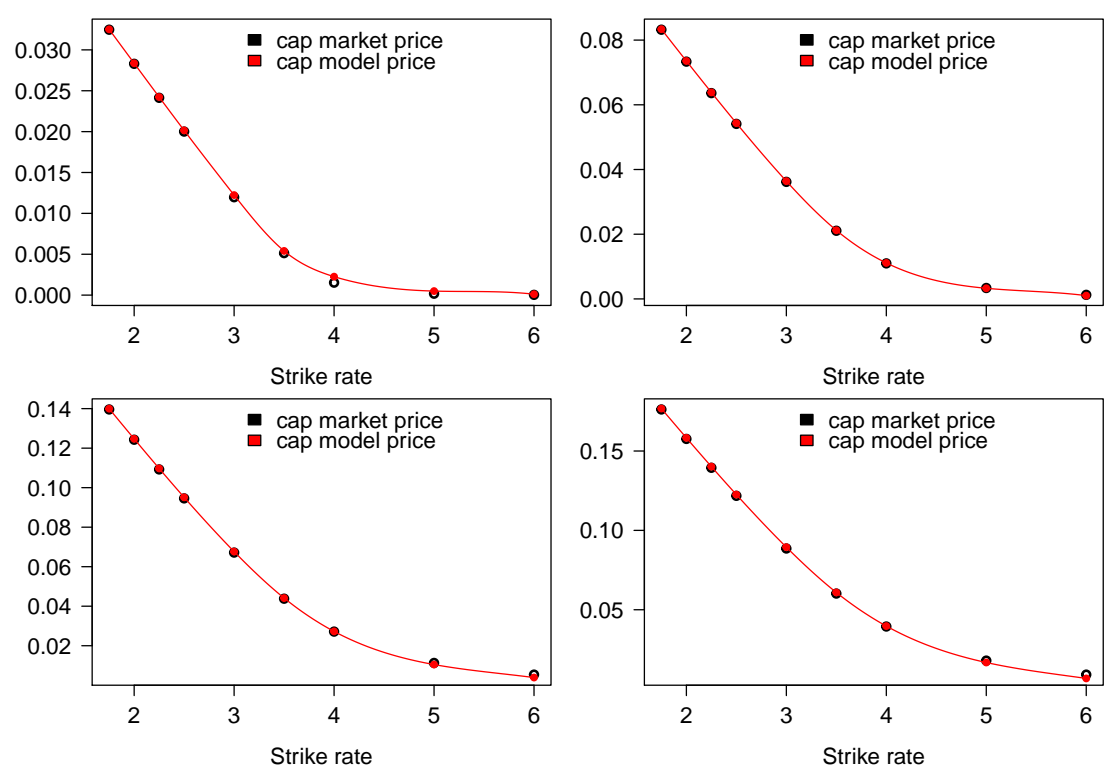

Figure 4.6.: Market and model (mid) prices of caps with maturity of two years (top left), five years (top right), eight years (bottom left) and ten years (bottom right) for strike rates of $1.75,2.00,2.25,2.50,3.00,3.50,4.00,5.00$ and 6.00 percent on $15^{\text {th }}$ August 2006 .

\subsubsection{Model Framework (II)}

Barndorff-Nielsen and Halgreen [6] studied the class of generalised hyperbolic (GH) distributions. In particular, they showed their infinite divisibility. Hence, each type of this distribution family with parameters $(\lambda, \alpha, \beta, \delta, \mu)$ satisfying $\lambda \in \mathbb{R}, 0 \leq|\beta|<\alpha, 0<\delta$ and $\mu \in \mathbb{R}$ generates a Lévy process (cf. Sato [81]). This process is called generalised hyperbolic Lévy motion with parameters $(\lambda, \alpha, \beta, \delta, \mu)$. A detailed discussion of this type of processes and its application in finance is given by Eberlein [28]. We also refer to the overview about jump-type Lévy processes given by Eberlein [29].

The normal inverse Gaussian (NIG) distribution is an important subclass of the GH distribution. It is obtained by setting the parameter $\lambda=-\frac{1}{2}$. The Lévy process that is generated by this distribution is called normal inverse Gaussian Lévy motion (see Eberlein [28, Section 5]). This type of process was first considered in finance by Barndorff-Nielsen [7].

This model approach is specified by the following volatility structure and driving process $(d=1)$ : 


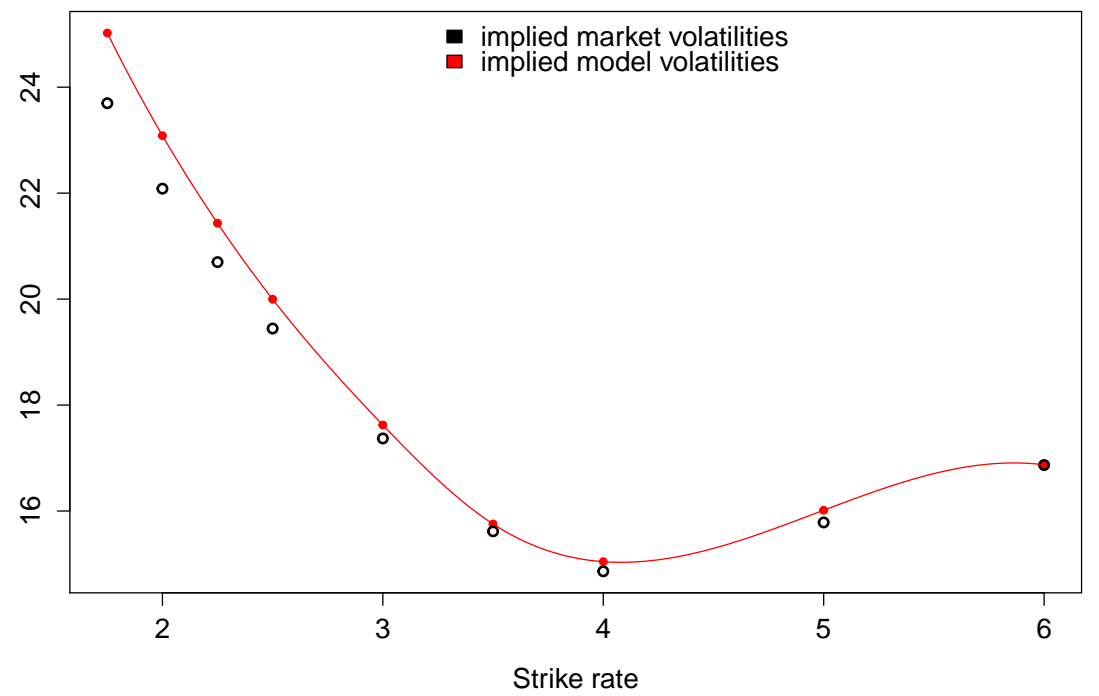

Figure 4.7.: Calibrated implied model volatilities and implied market volatilities for the maturity of 3.5 years on $15^{\text {th }}$ August 2006.

\begin{tabular}{lll}
\hline Maturity & Parameter & Objective function \\
\hline 1.25 Year & $4.5 \cdot 10^{-05}$ & $5.57 \cdot 10^{-09}$ \\
1.50 Year & 0.00018 & $3.96 \cdot 10^{-09}$ \\
1.75 Year & 0.00024 & $1.38 \cdot 10^{-09}$ \\
2.00 Year & 0.00036 & $1.76 \cdot 10^{-11}$ \\
\hline
\end{tabular}

Table 4.5.: Calibrated parameter $\gamma$ for different maturities.

(Vol.II) Vasicek volatility structure

$$
\sigma^{\mathrm{d}}(t, T)=\hat{\sigma} \exp (-a(T-t))
$$

with

$$
\Sigma^{\mathrm{d}}(t, T)= \begin{cases}\frac{\hat{\sigma}}{a}(1-\exp (-a(T-t))), & \text { when } t \leq T \\ 0, & \text { when } t>T\end{cases}
$$

(DP.II) Normal inverse Gaussian Lévy motion $L=\left(L_{t}\right)_{t \in\left[0, T^{*}\right]}$ defined on $\hat{\mathscr{B}}$.

The moment generating function of the normal inverse Gaussian distribution is given 


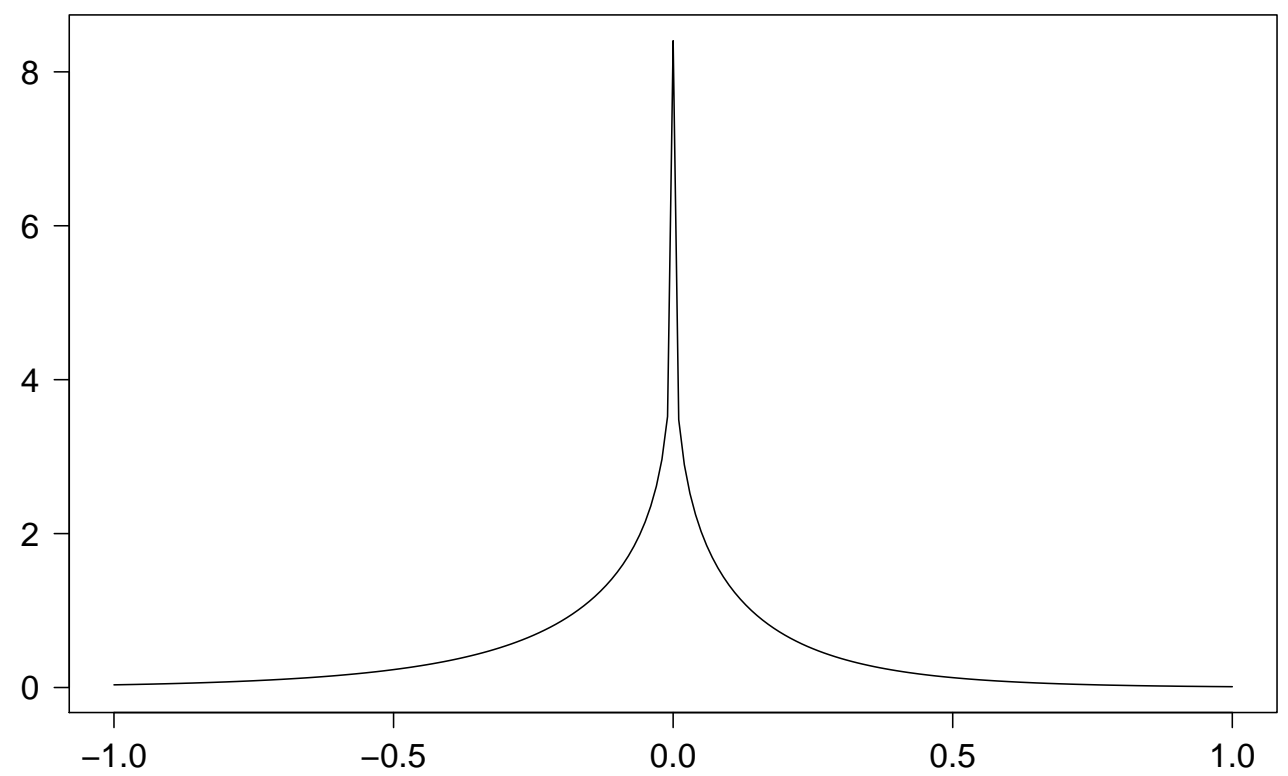

Figure 4.8.: Calibrated density of variance gamma distribution with parameter $\sigma=$ $0.2816519, \nu=1.724138$ and $\theta=-0.047200$.

by

$$
M_{\mathrm{NIG}(\alpha, \beta, \delta, \mu)}(u)=\frac{\exp \left(\mu u+\delta \sqrt{\alpha^{2}-\beta^{2}}\right)}{\exp \left(\delta \sqrt{\alpha^{2}-(\beta+u)^{2}}\right)}
$$

for every $u \in(-\alpha-\beta, \alpha-\beta)$. Consequently, we obtain the characteristic function as

$$
\varphi_{\mathrm{NIG}(\alpha, \beta, \delta, \mu)}(u)=\frac{\exp \left(i \mu u+\delta \sqrt{\alpha^{2}-\beta^{2}}\right)}{\exp \left(\delta \sqrt{\alpha^{2}-(\beta+i u)^{2}}\right)} .
$$

Note that we are able to extend the characteristic function on the set $\mathbb{R}-i(-\alpha-\beta, \alpha-\beta) \subset$ $\mathbb{C}$. It immediately follows from the explicit form of this function that

$$
L_{t} \sim \operatorname{NIG}(\alpha, \beta, t \delta, t \mu)
$$

for all $t \in\left(0, T^{*}\right]$.

We choose $R_{1}, \epsilon, M$ and $\hat{M}$ and deal with $\Sigma^{\mathrm{d}}$ in an analogous way as in the previous 


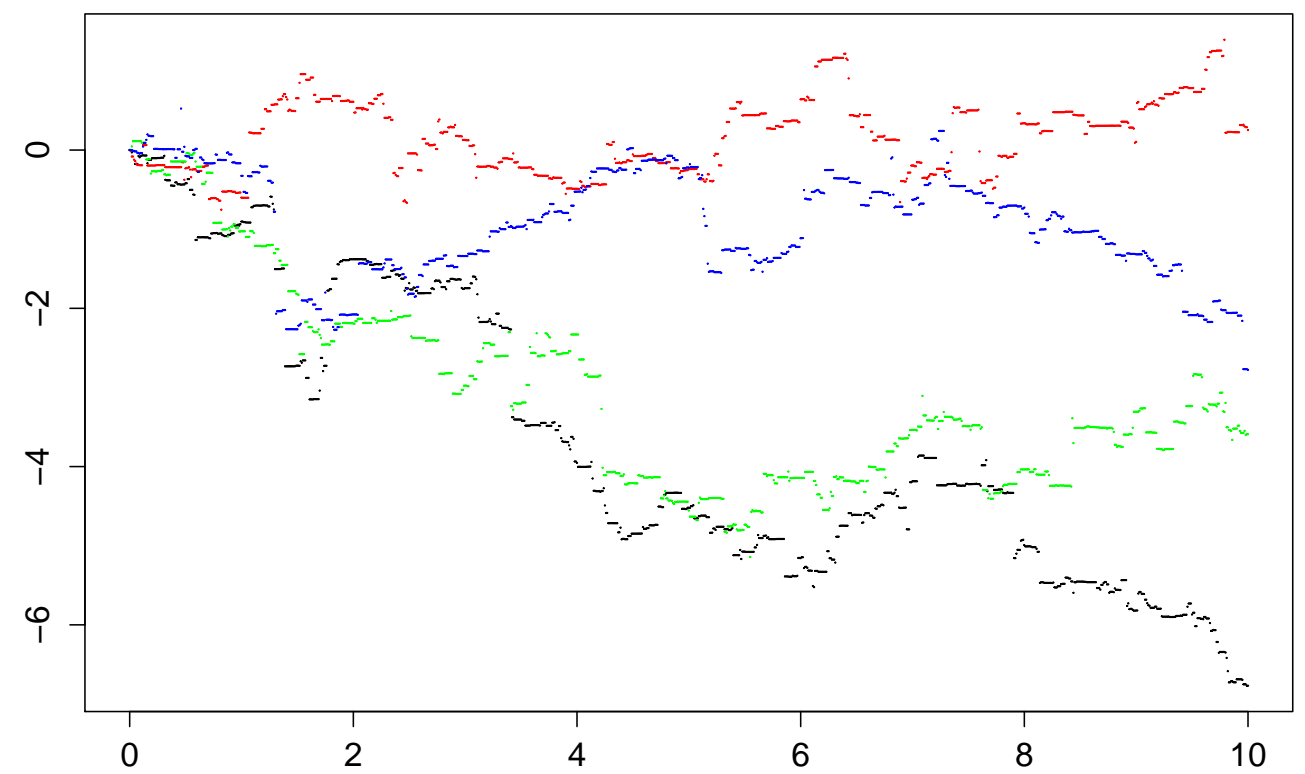

Figure 4.9.: Four sample paths of a variance gamma process with calibrated parameter.

framework. The extended cumulant $\theta_{s}$ of $L$ is determined by

$$
\theta_{s}(z)=\mu z+\delta \sqrt{\alpha^{2}-\beta^{2}}-\delta \sqrt{\alpha^{2}-(\beta+z)^{2}}
$$

for every $z \in \mathbb{C}$ with $|\operatorname{Re}(z)| \leq M$. From this term, we get the characteristic function of $X_{j}^{\mathrm{d}}$ under $P_{T_{j-1}}^{\mathrm{d}}$ at point $u-i R_{1}$ as

$$
\begin{aligned}
& \varphi_{X_{j}^{\text {d }}}^{T_{j-1}}\left(u-i R_{1}\right) \\
= & \exp \left(\int _ { 0 } ^ { T _ { j - 1 } } \left[-\mu \operatorname{sign}(a)\left(R_{1}+i u\right)\left[\exp \left(-a\left(T_{j}-s\right)\right)-\exp \left(-a\left(T_{j-1}-s\right)\right)\right]\right.\right. \\
& +\delta\left(\sqrt{\alpha^{2}-\left(\beta+\operatorname{sign}(a)\left(1-\exp \left(-a\left(T_{j-1}-s\right)\right)\right)\right)^{2}}\right. \\
& \left.\left.\left.-\sqrt{\alpha^{2}-\left(\beta+\chi_{j}\left(s, u, a, R_{1}\right)\right)^{2}}\right)\right] d s\right)
\end{aligned}
$$


where we set

$$
\begin{aligned}
\chi_{j}\left(s, u, a, R_{1}\right):= & \operatorname{sign}(a)\left(1-\exp \left(-a\left(T_{j-1}-s\right)\right)\right. \\
& \left.+\left(R_{1}+i u\right)\left[\exp \left(-a\left(T_{j-1}-s\right)\right)-\exp \left(-a\left(T_{j}-s\right)\right)\right]\right) .
\end{aligned}
$$

Observe that the deterministic part $D_{j}^{\mathrm{d}}$ results in

$$
\begin{aligned}
D_{j}^{\mathrm{d}}=\frac{B_{0}^{\mathrm{d}}\left(T_{j}\right)}{B_{0}^{\mathrm{d}}\left(T_{j-1}\right)} \exp ( & \int_{0}^{T_{j-1}} \mu \operatorname{sign}(a)\left[\exp \left(-a\left(T_{j}-s\right)\right)-\exp \left(-a\left(T_{j-1}-s\right)\right)\right] \\
& +\delta\left(\sqrt{\alpha^{2}-\left(\beta+\operatorname{sign}(a)\left(1-\exp \left(-a\left(T_{j}-s\right)\right)\right)\right)^{2}}\right. \\
& \left.\left.-\sqrt{\alpha^{2}-\left(\beta+\operatorname{sign}(a)\left(1-\exp \left(-a\left(T_{j-1}-s\right)\right)\right)\right)^{2}}\right) d s\right)
\end{aligned}
$$

and we obtain $\xi_{j}=-\ln D_{j}^{\mathrm{d}}$. Then, the caplet (mid) model price is given by

$$
\mathrm{Cpl}_{0}\left(T_{j-1}, T_{j}, K\right)=\frac{B_{0}^{\mathrm{d}}\left(T_{j-1}\right) \tilde{K}}{\pi} \cdot I_{j}\left(\alpha, \beta, \delta, a, R_{1}, K\right),
$$

where we define

$$
\begin{aligned}
& I_{j}\left(\alpha, \beta, \delta, a, R_{1}, K\right):=\int_{0}^{\infty} \operatorname{Re}\left(\left[\frac{B_{0}^{\mathrm{d}}\left(T_{j}\right)}{B_{0}^{\mathrm{d}}\left(T_{j-1}\right)}\right]^{R_{1}+i u} \frac{\tilde{K}^{-\left(1-R_{1}-i u\right)}}{\left(-R_{1}-i u\right)\left(1-R_{1}-i u\right)}\right. \\
& \times \exp \left(\int _ { 0 } ^ { T _ { j - 1 } } \delta \left(\sqrt{\alpha^{2}-\left(\beta+\operatorname{sign}(a)\left(1-\exp \left(-a\left(T_{j-1}-s\right)\right)\right)\right)^{2}}\right.\right. \\
& \left.-\sqrt{\alpha^{2}-\left(\beta+\chi_{j}\left(s, u, a, R_{1}\right)\right)^{2}}\right) \\
& +\left(R_{1}+i u\right) \delta\left(\sqrt{\alpha^{2}-\left(\beta+\operatorname{sign}(a)\left(1-\exp \left(-a\left(T_{j}-s\right)\right)\right)\right)^{2}}\right. \\
& \left.\left.\left.-\sqrt{\alpha^{2}-\left(\beta+\operatorname{sign}(a)\left(1-\exp \left(-a\left(T_{j-1}-s\right)\right)\right)\right)^{2}}\right) d s\right)\right) d u .
\end{aligned}
$$

\subsubsection{Calibration Results of Model Framework (II)}

The calibrated distribution parameters are presented in table 4.6 and the calibrated volatility structure parameter is given in table 4.7

The value of the objective function is

$$
O_{1}(\hat{\theta}, H)=2.39577 \cdot 10^{-06} \text {. }
$$

The market prices of caplets and caps for strike rates up to six percent are greatly calibrated as the figures 4.10 and 4.11 show. The relative errors of the market and model 


\begin{tabular}{ll}
\hline Parameter & Value \\
\hline$\alpha$ & 7.862 \\
$\beta$ & -6.508 \\
$\delta$ & 0.439909 \\
\hline
\end{tabular}

Table 4.6.: Calibrated parameter of normal inverse Gaussian distribution.

\begin{tabular}{ll}
\hline Parameter & Value \\
\hline$a$ & 0.0119 \\
\hline
\end{tabular}

Table 4.7.: Calibrated parameter of Vasicek volatility structure.

prices are presented in figures $\mathrm{B} .1$ and $\mathrm{B} .2$ in the appendix. As in the previous framework, the divergences for short maturities and strike rates over six percent result from the very small values of the prices. Figure 4.12 illustrates the fit of the volatility smile for the maturity of 3.5 years.

The density function is plotted in figure 4.13 and four paths of the driving process are illustrated in figure 4.14 .

\subsection{Calibration of Caps in the Two-Price and Multiple-Curve Setting}

Now we consider market cap volatilities quoted during the financial crisis on $15^{\text {th }}$ September 2009. Therefore, we need to apply the multiple-curve approach. We proceed with the given data sets as described in section 4.1. Recall that quotes up to a maturity of two years are indexed on the three-month Euribor and quotes larger than 2 years are related to the six-month Euribor.

\subsubsection{Data Sets}

The initial discount bond prices are given in table 4.8 and 4.9 . The edited surfaces of the quoted cap (mid) implied volatilities based on the three-month and six-month tenor are plotted in figures 4.15 and 4.16 . In figures 4.17 and 4.18 , we present the ask and bid spreads between the implied (spot) volatilities of caplets for the relevant maturities and strike rates. As we can see the spreads are significantly larger than in the pre-crisis data sets (cf. figures 4.3 and 4.4 . 

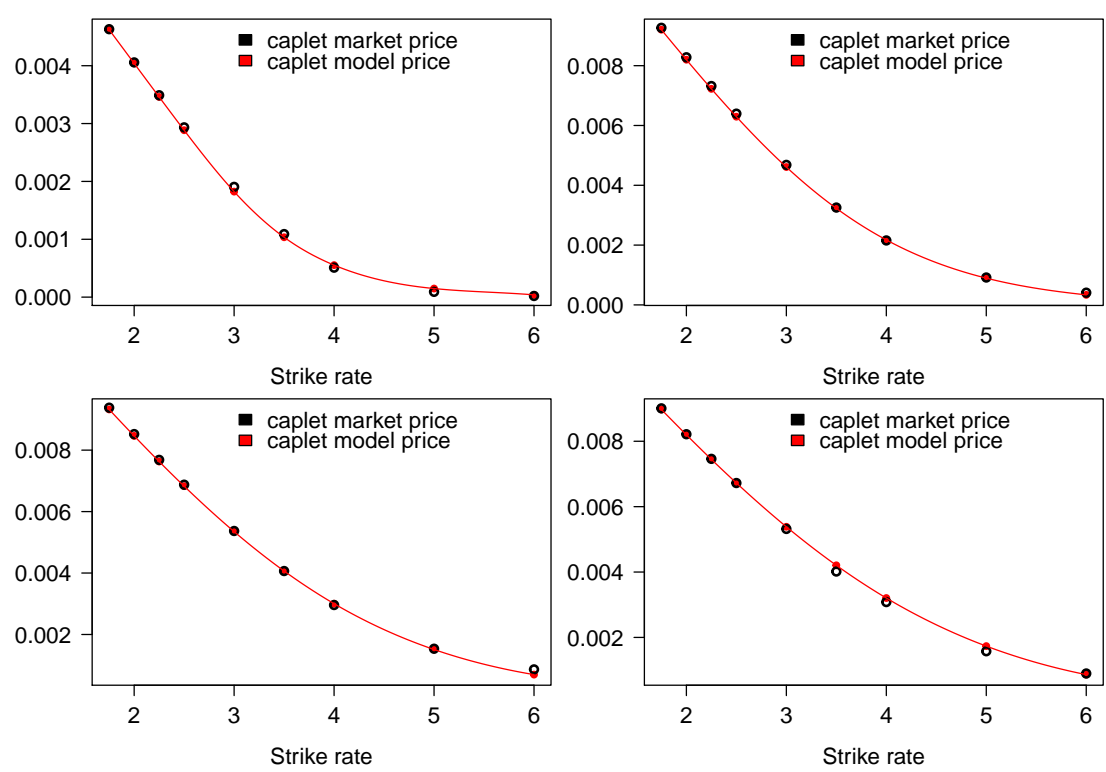

Figure 4.10.: Market and model (mid) prices of caplets with maturity of two years (top left), five years (top right), eight years (bottom left) and ten years (bottom right) for strike rates of 1.75, 2.00, 2.25, 2.50, 3.00, 3.50, 4.00, 5.00 and 6.00 percent on $15^{\text {th }}$ August 2006.

\subsubsection{Calibration Procedure}

We proceed as it is described in section 2.4, where we consider

$$
\begin{aligned}
H=\{(T, H): & T \in\{1,1.25,1.5,2,2.5, \ldots, 5.5,6\} \\
& K \in\{0.0175,0.02,0.0225,0.025,0.03,0.035,0.04,0.05\}\}
\end{aligned}
$$

and

$$
H_{T}=\{(T, K): K \in\{0.0175,0.02,0.0225,0.025,0.03\}\}
$$

for $T=3$. Here, we have $T^{*}=6$.

\subsubsection{Model Framework}

We design the model in such a manner as it is stated in section 3.2 This approach guarantees the monotonicity of the curves. Note that we consider three term structures: the discount curve, the three-month curve and the six-month curve.

The volatility function, the liquidity function and the driving process are specified as follows $(d=3, m=2$ and $l=1)$ : 

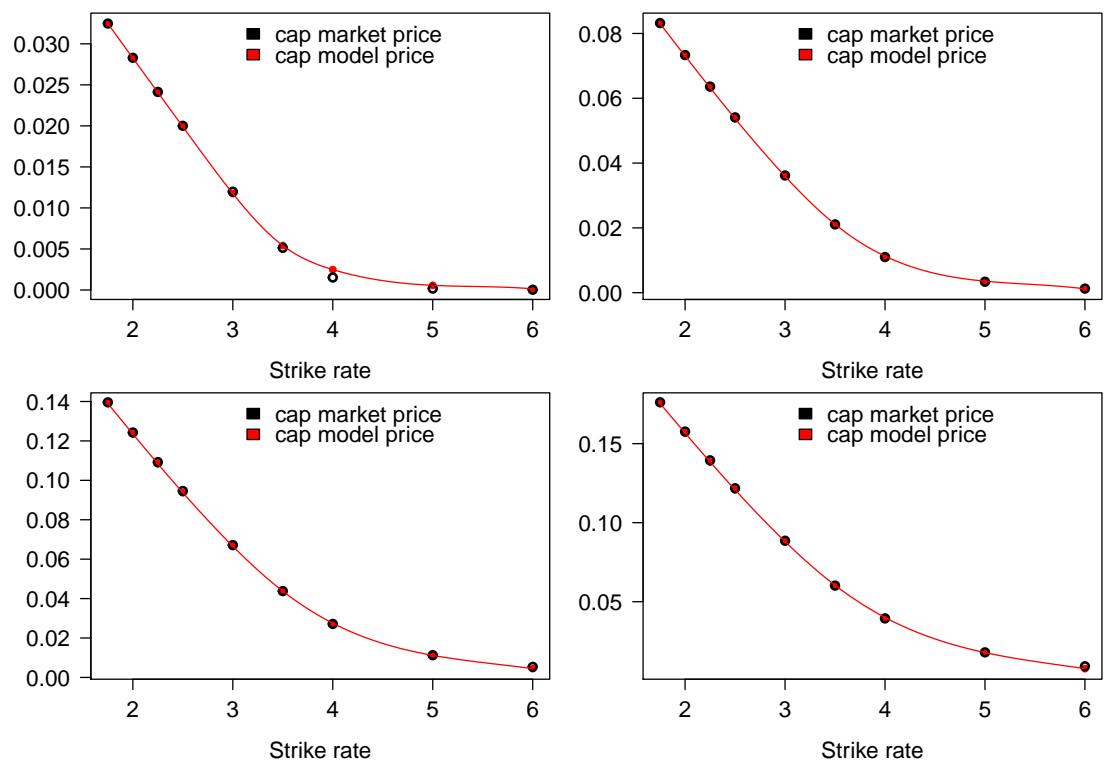

Figure 4.11.: Market and model (mid) prices of caps with maturity of two years (top left), five years (top right), eight years (bottom left) and ten years (bottom right) for strike rates of $1.75,2.00,2.25,2.50,3.00,3.50,4.00,5.00$ and 6.00 percent on $15^{\text {th }}$ August 2006.

(Vol) Vasicek volatility structure:

- Discount curve

$$
\sigma^{\mathrm{d}}(t, T)=\left(\begin{array}{c}
\hat{\sigma}_{\mathrm{d}} \exp \left(-a_{\mathrm{d}}(T-t)\right) \\
0 \\
0
\end{array}\right)^{\top}
$$

with

$$
\Sigma_{1}^{\mathrm{d}}(t, T)= \begin{cases}\frac{\hat{\sigma}_{\mathrm{d}}}{a_{\mathrm{d}}}\left(1-\exp \left(-a_{\mathrm{d}}(T-t)\right)\right), & \text { when } t \leq T \\ 0, & \text { when } t>T\end{cases}
$$

and $\Sigma_{2}^{\mathrm{d}}(t, T)=\Sigma_{3}^{\mathrm{d}}(t, T)=0$ for any $t, T \in\left[0, T^{*}\right]$, where $\hat{\sigma}_{\mathrm{d}}>0$ and $a_{\mathrm{d}} \neq 0$.

- Spreads related to multiple term structures: 
4.3. Calibration of Caps in the Two-Price and Multiple-Curve Setting

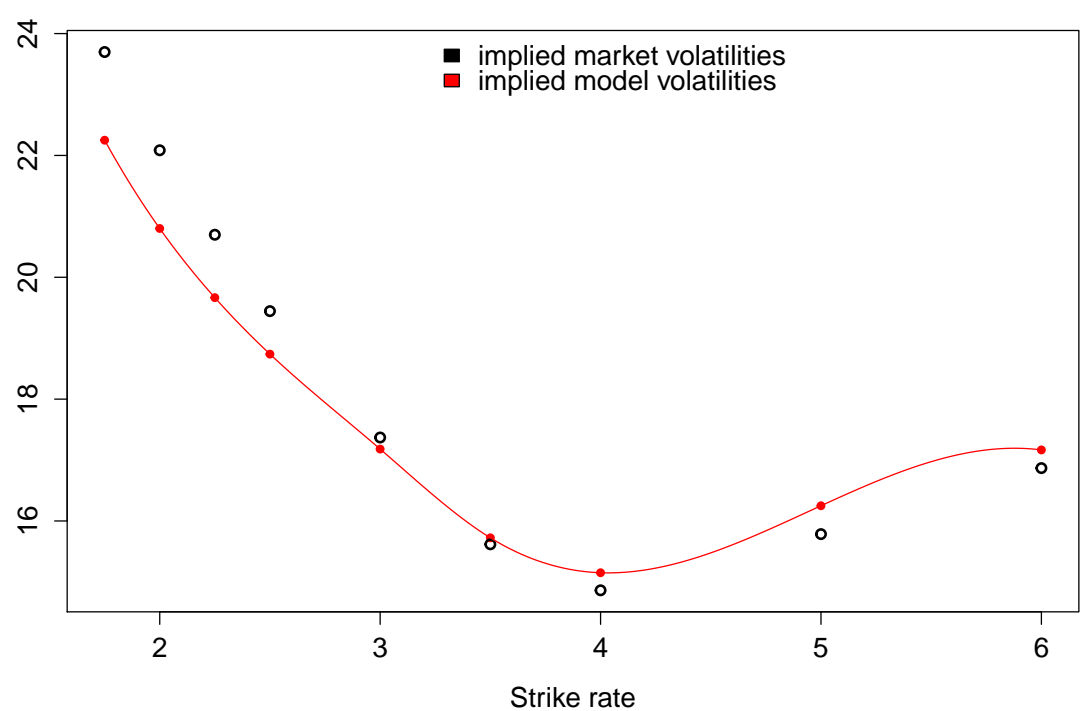

Figure 4.12.: Calibrated implied model volatilities and implied market volatilities for the maturity of 3.5 years on $15^{\text {th }}$ August 2006 .

(1) Spread between curve $d$ and 1:

$$
\sigma^{1, \mathrm{~d}}(t, T)=\left(\begin{array}{c}
0 \\
\hat{\sigma}_{1 \mathrm{~d}} \exp \left(-a_{1 \mathrm{~d}}(T-t)\right) \\
0
\end{array}\right)^{\top}
$$

with

$$
\Sigma_{2}^{1, \mathrm{~d}}(t, T)= \begin{cases}\frac{\hat{\sigma}_{1 \mathrm{~d}}}{a_{1 \mathrm{~d}}}\left(1-\exp \left(-a_{1 \mathrm{~d}}(T-t)\right)\right), & \text { when } t \leq T \\ 0, & \text { when } t>T\end{cases}
$$

and $\Sigma_{1}^{1, \mathrm{~d}}(t, T)=\Sigma_{3}^{1, \mathrm{~d}}(t, T)=0$ for every $t, T \in\left[0, T^{*}\right]$, with $\hat{\sigma}_{1 \mathrm{~d}}>0$ and $a_{1 \mathrm{~d}} \neq 0$.

(2) Spread between curve 1 and 2:

$$
\sigma^{2,1}(t, T)=\left(\begin{array}{c}
0 \\
0 \\
\hat{\sigma}_{21} \exp \left(-a_{21}(T-t)\right)
\end{array}\right)^{\top}
$$




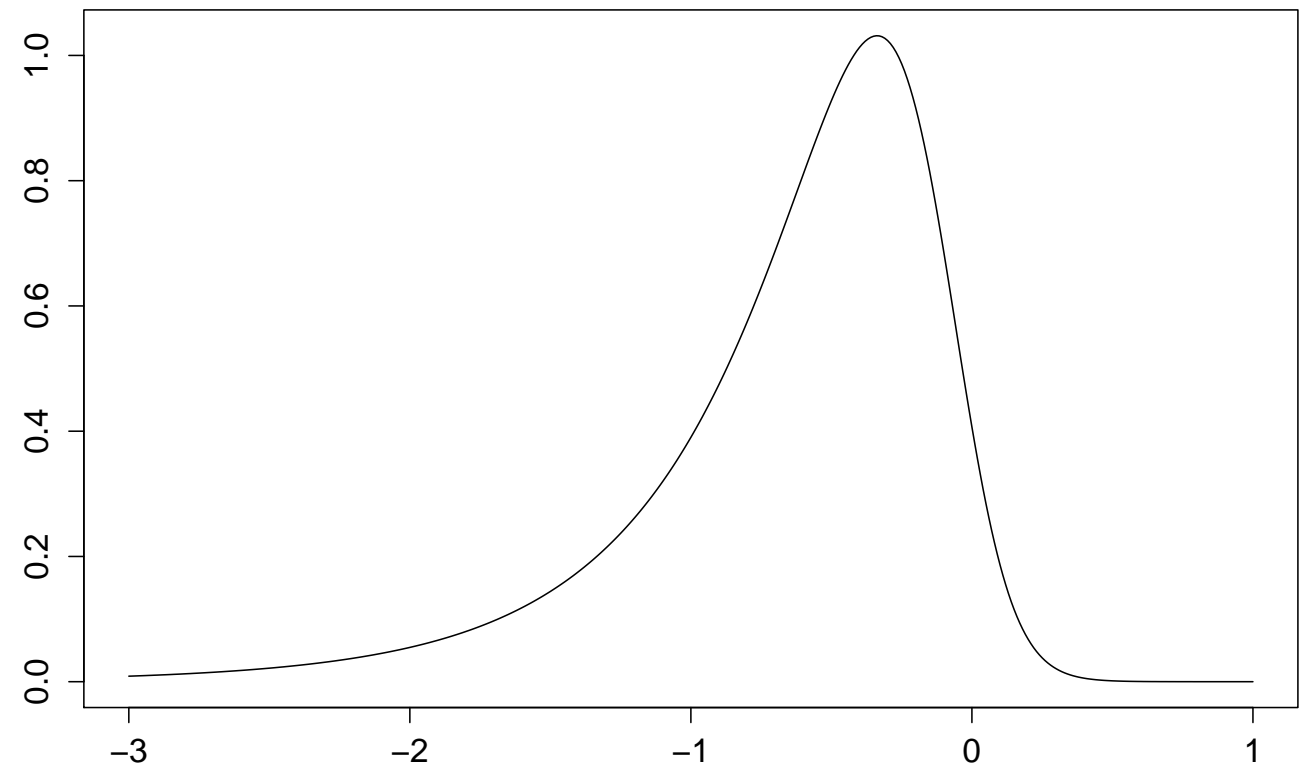

Figure 4.13.: Density function of normal inverse Gaussian distribution for the calibrated parameter.

with

$$
\Sigma_{3}^{2,1}(t, T)= \begin{cases}\frac{\hat{\sigma}_{21}}{a_{21}}\left(1-\exp \left(-a_{21}(T-t)\right)\right), & \text { when } t \leq T \\ 0, & \text { when } t>T\end{cases}
$$

and $\Sigma_{1}^{2,1}(t, T)=\Sigma_{2}^{2,1}(t, T)=0$ for all $t, T \in\left[0, T^{*}\right]$, where $\hat{\sigma}_{21}>0$ and $a_{21} \neq 0$.

(L) Liquidity function (see Brigo and Mercurio [17]):

$$
l^{j}(t, T)=\sigma^{j} \cdot(T-t) \exp \left(-b^{j}(T-t)\right)
$$

where $\sigma^{j}, b^{j}>0$ for any $j \in\{1,2\}$.

As in section 4.2, we can choose $\hat{\sigma}_{\mathrm{d}}=\left|a_{\mathrm{d}}\right|, \hat{\sigma}_{1 \mathrm{~d}}=\left|a_{1 \mathrm{~d}}\right|$, and $\hat{\sigma}_{21}=\left|a_{21}\right|$. For $t, T \in$ 


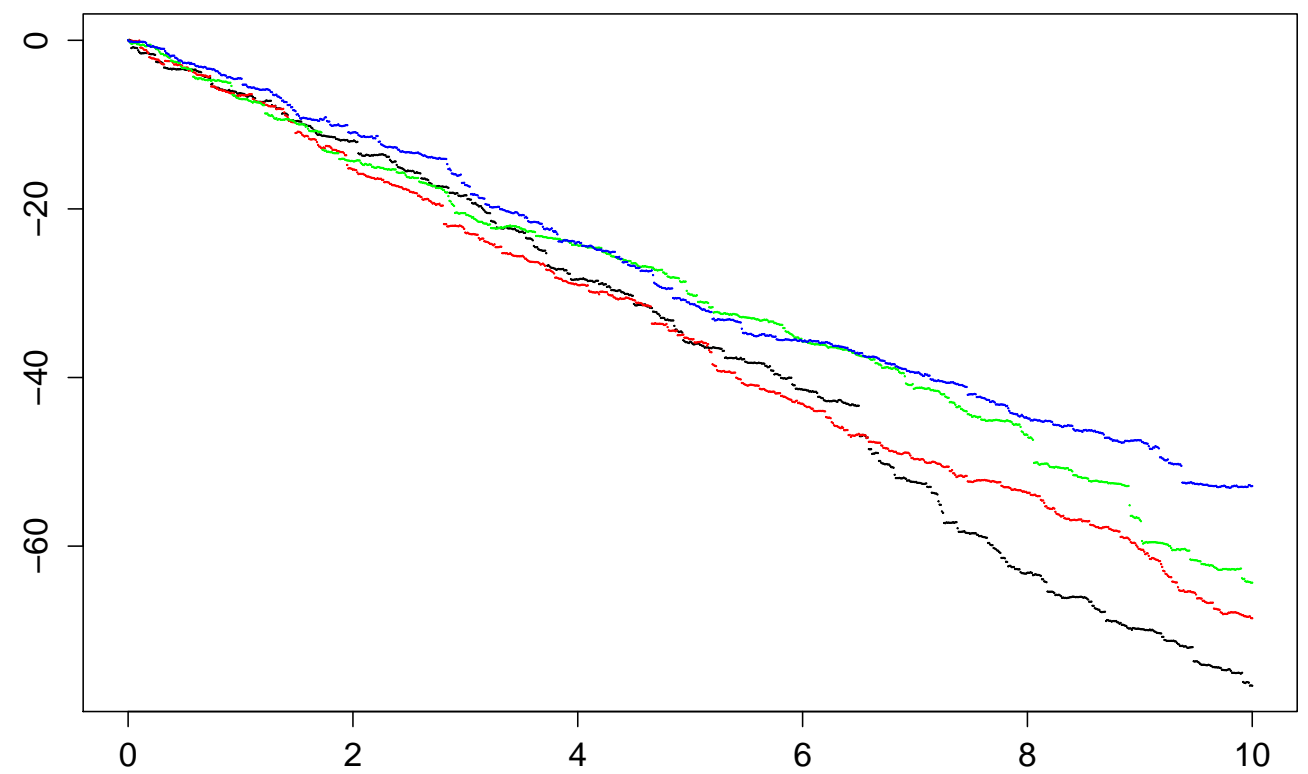

Figure 4.14.: Four sample paths of a normal inverse Gaussian process with calibrated parameter.

$\left[0, T^{*}\right]$ with $t \leq T$, we therefore obtain

$$
\Sigma^{1}(t, T)=\Sigma^{\mathrm{d}}(t, T)+\Sigma^{1, \mathrm{~d}}(t, T)=\left(\begin{array}{c}
\operatorname{sign}\left(a_{\mathrm{d}}\right)\left(1-\exp \left(-a_{\mathrm{d}}(T-t)\right)\right) \\
\operatorname{sign}\left(a_{1 \mathrm{~d}}\right)\left(1-\exp \left(-a_{1 \mathrm{~d}}(T-t)\right)\right) \\
0
\end{array}\right)^{\top}
$$

and

$$
\begin{aligned}
\Sigma^{2}(t, T) & =\Sigma^{\mathrm{d}}(t, T)+\Sigma^{1, \mathrm{~d}}(t, T)+\Sigma^{2,1}(t, T) \\
& =\left(\begin{array}{c}
\operatorname{sign}\left(a_{\mathrm{d}}\right)\left(1-\exp \left(-a_{\mathrm{d}}(T-t)\right)\right) \\
\operatorname{sign}\left(a_{1 \mathrm{~d}}\right)\left(1-\exp \left(-a_{1 \mathrm{~d}}(T-t)\right)\right) \\
\operatorname{sign}\left(a_{21}\right)\left(1-\exp \left(-a_{21}(T-t)\right)\right)
\end{array}\right)^{\top} .
\end{aligned}
$$

In accordance with assumption $(\mathbb{M C} . \mathbb{D E T})$, we require the parameter $a_{\mathrm{d}}, a_{1 \mathrm{~d}}$ and $a_{21}$ to be restricted to values such that the volatility function $\Sigma^{2}$ is bounded in each component by a constant $\hat{M}$ satisfying $0<\hat{M}<M$. More specifically, we claim the existence of the cumulant function $\theta$ at $\Sigma^{2}(t, T)$ for all $t, T \in\left[0, T^{*}\right]$ with $t \leq T$. Then, it also exists at 


\begin{tabular}{ll}
\hline Maturity & Initial Bond Price \\
\hline 0.25 Year & 0.9990052 \\
0.50 Year & 0.9979506 \\
0.75 Year & 0.9960934 \\
1.00 Year & 0.9932698 \\
1.25 Year & 0.9895302 \\
1.50 Year & 0.9849790 \\
1.75 Year & 0.9797332 \\
2.00 Year & 0.9739065 \\
\hline
\end{tabular}

Table 4.8.: Discount bond prices $B_{0}^{\mathrm{d}}(T)$ on $15^{\text {th }}$ September 2009.

$\Sigma^{\mathrm{d}}(t, T)$ and $\Sigma^{1}(t, T)$. Clearly, the parameters of the liquidity function can be restricted to a set such that the liquidity function is bounded. Observe that the considered volatility and liquidity functions satisfy assumption $(\mathbb{V} \mathbb{L})$. To ensure the drift condition $(3.26)$, we have to choose the drift terms $A^{1, \mathrm{~d}}$ and $A^{2,1}$ as

$$
A^{1, \mathrm{~d}}(t, T)=\theta\left(\Sigma^{1}(t, T)\right)-\theta\left(\Sigma^{\mathrm{d}}(t, T)\right)+l^{1}(t, T)
$$

and

$$
A^{2,1}(t, T)=\theta\left(\Sigma^{2}(t, T)\right)-\theta\left(\Sigma^{1}(t, T)\right)+l^{21}(t, T) .
$$

(DP) The driving process $L=\left(L^{1}, L^{2}, L^{3}\right)^{\top}$ defined on $\left(\Omega, \mathscr{G}, \mathbb{G}, P^{\mathrm{d}}\right)$ is constructed as follows:

(i) $N$ is a normal inverse Gaussian Lévy motion with parameters $\alpha, \beta, \delta, \mu$ satisfying $0 \leq|\beta|\left\langle\alpha, \delta>0\right.$ and $\mu \in \mathbb{R}$ and $Z^{j}$ is a Gamma process with parameters $\alpha_{j}, \beta_{j}>0$ for any $j \in\{1,2,3\}$.

(ii) $N, Z^{1}, Z^{2}$ and $Z^{3}$ are assumed to be stochastically independent.

(iii) $Y^{1}:=L^{1}=N+Z^{3}$.

(iv) $Y^{2}:=\left(L^{2}, L^{3}\right)^{\top}=-\left(Z^{1}+Z^{3}, Z^{2}+Z^{3}\right)^{\top}$.

Clearly, the components $L^{1}, L^{2}$ and $L^{3}$ are stochastically dependent. Further, the processes $N, Z^{1}, Z^{2}, Z^{3}$ do not possess a continuous local martingale part.

Let us define the vector process $V:=\left(N, Z^{1}, Z^{2}, Z^{3}\right)^{\top}$. The following Lemma states similar results as noted by Crépey, Grbac, and Nguyen [23, Example 3.6] and is analogously proved.

Lemma 4.3.1 1. The Lévy measure $F^{V}$ of $V$ is given by

$$
F^{V}(A)=F^{N}\left(A_{1}\right)+F^{Z^{1}}\left(A_{2}\right)+F^{Z^{2}}\left(A_{3}\right)+F^{Z^{3}}\left(A_{4}\right),
$$




\begin{tabular}{ll}
\hline Maturity & Initial Bond Price \\
\hline 0.50 Year & 0.9979506 \\
1.00 Year & 0.9932698 \\
1.50 Year & 0.9849790 \\
2.00 Year & 0.9739065 \\
2.50 Year & 0.9607654 \\
3.00 Year & 0.9460177 \\
3.50 Year & 0.9300146 \\
4.00 Year & 0.9130396 \\
4.50 Year & 0.8952878 \\
5.00 Year & 0.8769590 \\
5.50 Year & 0.8582155 \\
6.00 Year & 0.8392186 \\
6.50 Year & 0.8200582 \\
7.00 Year & 0.8009114 \\
7.50 Year & 0.7818049 \\
8.00 Year & 0.7628484 \\
8.50 Year & 0.7441259 \\
9.00 Year & 0.7256300 \\
9.50 Year & 0.7074524 \\
10.0 Year & 0.6896507 \\
\hline
\end{tabular}

Table 4.9.: Discount bond prices $B_{0}^{\mathrm{d}}(T)$ on $15^{\text {th }}$ September 2009 .

where $A \in \mathcal{B}\left(\mathbb{R}^{4}\right)$ and we set $A_{k}:=\left\{x \in \mathbb{R} \mid x e_{k} \in A\right\}$ for any $k \in\{1,2,3,4\}$ with unit vector $e_{k}$ in $\mathbb{R}^{4}$ that has the entry one in the $k^{\text {th }}$-component and zero otherwise.

2. The Lévy measure $F$ of $L$ is given by

$$
\begin{aligned}
F(B)= & F^{N}\left(\left\{x \in \mathbb{R} \mid(x, 0,0)^{\top} \in B\right\}\right) \\
& +F^{Z^{1}}\left(\left\{x \in \mathbb{R} \mid(0,-x, 0)^{\top} \in B\right\}\right)+F^{Z^{2}}\left(\left\{x \in \mathbb{R} \mid(0,0,-x)^{\top} \in B\right\}\right) \\
& +F^{Z^{3}}\left(\left\{x \in \mathbb{R} \mid(x,-x,-x)^{\top} \in B\right\}\right),
\end{aligned}
$$

where $B \in \mathcal{B}\left(\mathbb{R}^{3} \backslash\{0\}\right)$. Moreover, the process $-Y^{2}$ is a subordinator.

Proof: Since $N, Z^{1}, Z^{2}$ and $Z^{3}$ are independent, the first assertion is a consequence of Sato [81, Exercise 12.10]: The components $X^{1}, \ldots, X^{p}$ of an $\mathbb{R}^{p}$-valued random variable $X=\left(X^{1}, \ldots, X^{p}\right)^{\top}$ with infinitely divisible distribution and generating triplet $(A, \nu, \gamma)$ (see Sato [81, chapter 2, section 8]) are independent if and only if $A$ is in diagonal form and $\nu$ is supported on the union of the coordinate axes. For the sake of completeness, we prove the statement of the exercise for our situation $(A=0$ and $p=4)$ : 


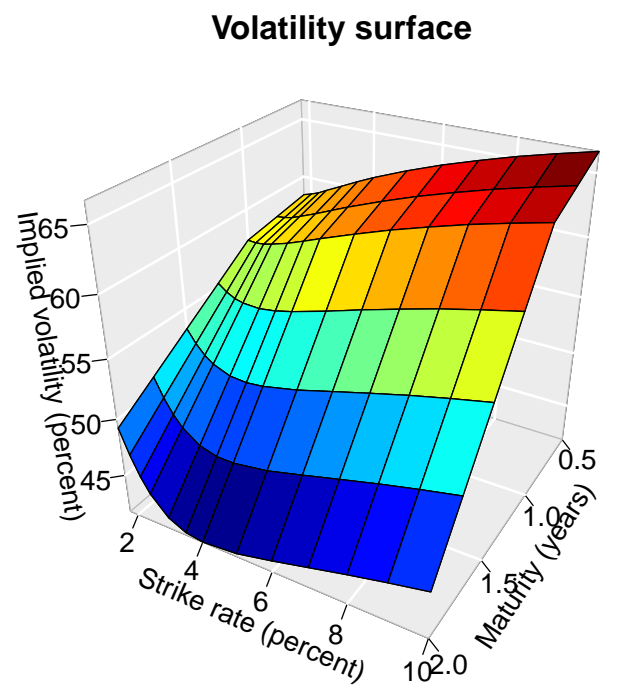

Figure 4.15.: (Mid) implied volatility surface based on the three-month tenor.

Let us suppose that $F^{V}$ is supported by the union of the coordinate axes. This means that we have

$$
F^{V}(A)=\sum_{k=1}^{4} \hat{F}_{k}\left(A_{k}\right),
$$

where $A \in \mathcal{B}\left(\mathbb{R}^{4}\right)$ and $\hat{F}_{k}$ is a measure for each $k \in\{1, \ldots, 4\}$. One easily concludes from Sato [81, Proposition 11.10] that $\hat{F}_{k}=F^{V^{k}}$ for every $k \in\{1, \ldots, 4\}$. Then, we have

$$
\begin{aligned}
\mathbb{E}_{P^{\mathrm{d}}}\left[\exp \left(i\left\langle u, V_{t}\right\rangle\right)\right] & =\exp \left(t\left(i\langle b, u\rangle+\int_{\mathbb{R}^{4}}\left(e^{i\langle u, x\rangle}-1-i\langle u, h(x)\rangle\right) F^{V}(d x)\right)\right) \\
& =\exp \left(t \sum_{k=1}^{4}\left(i b_{k} u_{k}+\int_{\mathbb{R}}\left(e^{i u_{k} x_{k}}-1-i u_{k} h^{k}\left(x_{k}\right)\right) F^{V^{k}}\left(d x_{k}\right)\right)\right) \\
& =\prod_{k=1}^{4} \mathbb{E}_{P^{\mathrm{d}}}\left[\exp \left(i u_{k} V_{t}^{k}\right)\right]
\end{aligned}
$$

for some truncation function $h \in \mathscr{C}_{t}^{4}$. This shows the independence of the components of the Lévy process $V$. For the converse statement let us define a measure $\hat{F}$ on $\left(\mathbb{R}^{4}, \mathcal{B}\left(\mathbb{R}^{4}\right)\right)$ 


\section{Volatility surface}

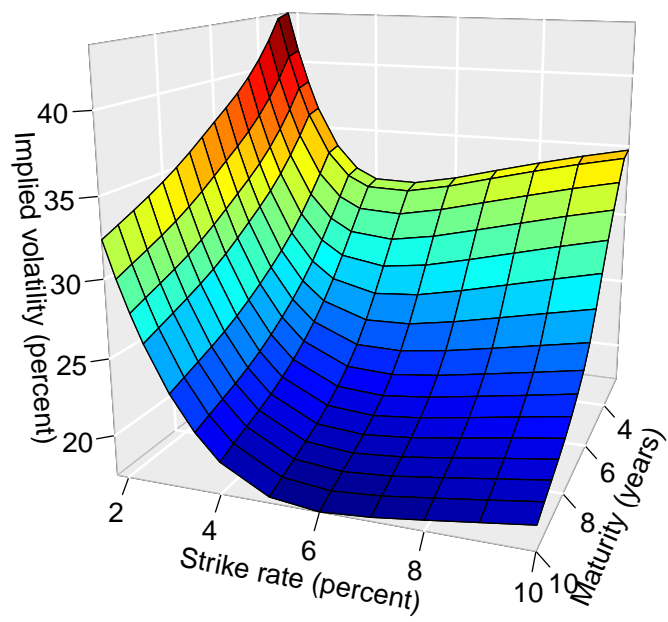

Figure 4.16.: (Mid) implied volatility surface based on the six-month tenor.

by

$$
\hat{F}(A):=\sum_{k=1}^{4} F^{V^{k}}\left(A_{k}\right) .
$$

Clearly, it holds $\hat{F}(\{0\})=0$ and

$$
\int_{\mathbb{R}^{4}}\left(|x|^{2} \wedge 1\right) \hat{F}(d x)=\sum_{k=1}^{4} \int_{\mathbb{R}}\left(\left|x_{k}\right|^{2} \wedge 1\right) F^{V^{k}}\left(d x_{k}\right)<\infty .
$$

Therefore, it follows that $\hat{F}$ is a Lévy measure. By using the independence of the components, we obtain

$$
\begin{aligned}
\mathbb{E}_{P^{\mathrm{d}}}\left[\exp \left(i\left\langle u, V_{t}\right\rangle\right)\right] & =\exp \left(t \sum_{k=1}^{4}\left(i b_{k} u_{k}+\int_{\mathbb{R}}\left(e^{i u_{k} x_{k}}-1-i u_{k} h^{k}\left(x_{k}\right)\right) F^{V^{k}}\left(d x_{k}\right)\right)\right) \\
& =\exp \left(t\left(i\langle b, u\rangle+\int_{\mathbb{R}^{4}}\left(e^{i\langle u, x\rangle}-1-i\langle u, h(x)\rangle\right) \hat{F}(d x)\right)\right)
\end{aligned}
$$

for some $h \in \mathscr{C}_{t}^{4}$. On the basis of the uniqueness of this representation, we conclude that 


\section{Caplet volatility spread}

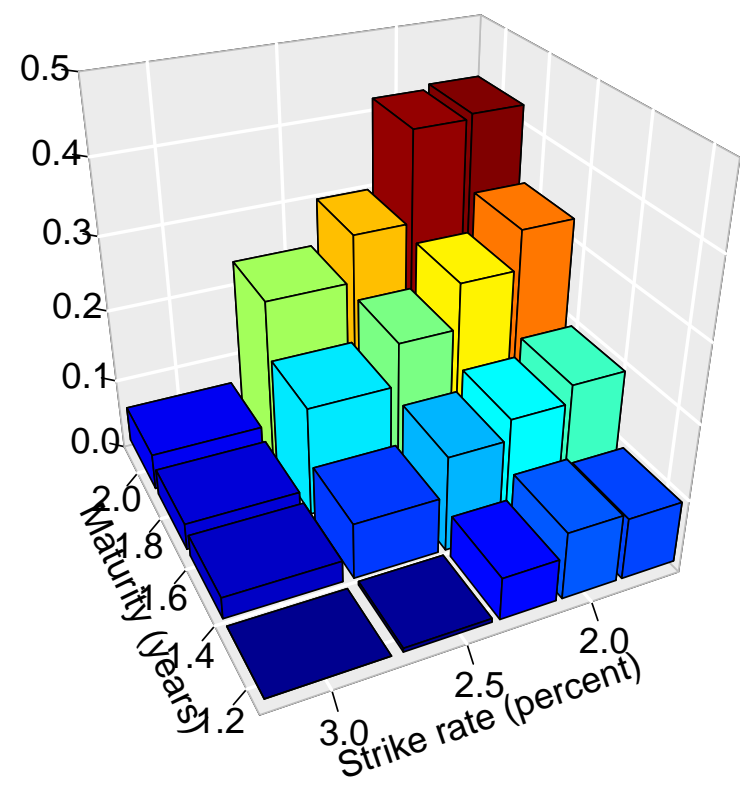

Figure 4.17.: Spread between ask and bid (spot) volatilities of caplets on $15^{\text {th }}$ September 2009. We consider maturities of $1.25,1.5,1.75$ and 2 years and strike rates of $1.75,2,2.25,2.5,3$ percent.

$\hat{F}=F^{V}$. For the second claim, we define the matrix

$$
U:=\left(\begin{array}{cccc}
1 & 0 & 0 & 1 \\
0 & -1 & 0 & -1 \\
0 & 0 & -1 & -1
\end{array}\right)
$$

such that $L=U V$. Then, by Sato [81, Proposition 11.10], we conclude that

$$
\begin{aligned}
F(B)= & F^{V}\left(\left\{x \in \mathbb{R}^{4} \mid U x \in B\right\}\right) \\
= & F^{V}\left(\left\{x \in \mathbb{R}^{4} \mid\left(x_{1}+x_{4},-x_{2}-x_{4},-x_{3}-x_{4}\right)^{\top} \in B\right\}\right) \\
= & F^{N}\left(\left\{x \in \mathbb{R} \mid(x, 0,0)^{\top} \in B\right\}\right) \\
& +F^{Z^{1}}\left(\left\{x \in \mathbb{R} \mid(0,-x, 0)^{\top} \in B\right\}\right)+F^{Z^{2}}\left(\left\{x \in \mathbb{R} \mid(0,0,-x)^{\top} \in B\right\}\right) \\
& +F^{Z^{3}}\left(\left\{x \in \mathbb{R} \mid(x,-x,-x)^{\top} \in B\right\}\right),
\end{aligned}
$$




\section{Caplet volatility spread}

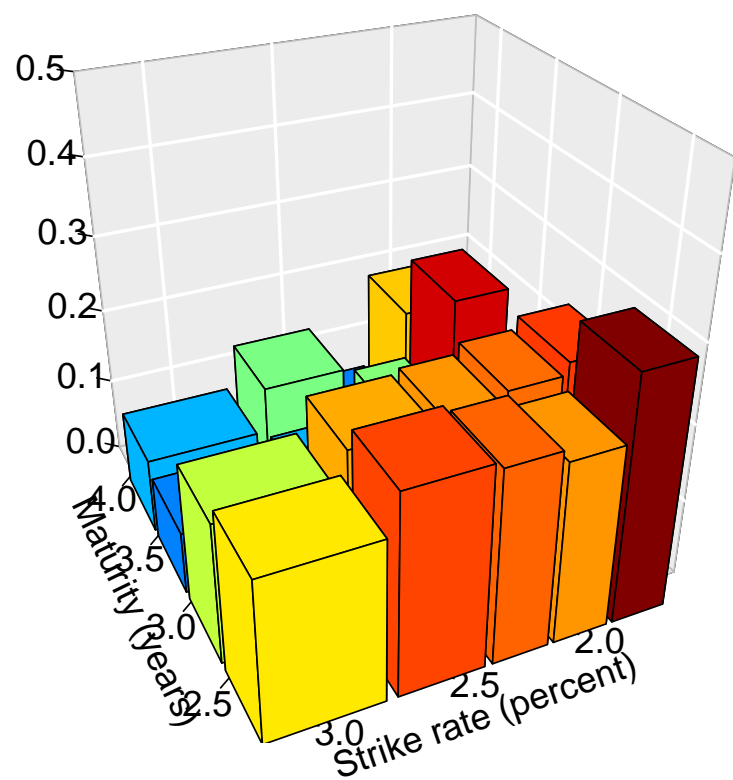

Figure 4.18.: Spread between ask and bid (spot) volatilities of caplets on $15^{\text {th }}$ September 2009. We consider maturities of 2.5,3,3.5 and 4 years and strike rates of $1.75,2,2.25,2.5,3$ percent.

where $B \in \mathcal{B}\left(\mathbb{R}^{3} \backslash\{0\}\right)$.

To ensure the positivity of the forward spreads, we get the following deterministic restrictions on the model parameters (see Proposition 3.2.2 and conditions (3.36) and (3.37): For every $T \in\left[0, T^{*}\right]$, we assume that

$$
\theta\left(\Sigma^{1}(t, T)\right)-\theta\left(\Sigma^{\mathrm{d}}(t, T)\right)+l^{1}(t, T) \leq s_{0}^{1, \mathrm{~d}}(T)+\theta\left(\Sigma^{1}(0, T)\right)-\theta\left(\Sigma^{\mathrm{d}}(0, T)\right)+l^{1}(0, T)
$$

and

$$
\theta\left(\Sigma^{2}(t, T)\right)-\theta\left(\Sigma^{1}(t, T)\right)+l^{2,1}(t, T) \leq s_{0}^{2,1}(T)+\theta\left(\Sigma^{2}(0, T)\right)-\theta\left(\Sigma^{1}(0, T)\right)+l^{2,1}(0, T)
$$

for all $t \in[0, T]$. Then, we conclude that the desired monotonicity 3.27 is satisfied.

By using the stochastic independence of the processes $N, Z^{1}, Z^{2}$ and $Z^{3}$, we can 


\section{Model Calibration}

express the cumulant function $\theta$ of $L$ under measure $P^{\mathrm{d}}$ as

$$
\begin{aligned}
\theta(z)= & \theta^{N}\left(z_{1}\right)+\theta^{Z^{1}}\left(-z_{2}\right)+\theta^{Z^{2}}\left(-z_{3}\right)+\theta^{Z^{3}}\left(z_{1}-z_{2}-z_{3}\right) \\
= & \mu z_{1}+\delta \sqrt{\alpha^{2}-\beta^{2}}-\delta \sqrt{\alpha^{2}-\left(\beta+z_{1}\right)^{2}}-\beta_{1} \log \left(1+\frac{z_{2}}{\alpha_{1}}\right) \\
& -\beta_{2} \log \left(1+\frac{z_{3}}{\alpha_{2}}\right)-\beta_{3} \log \left(1-\frac{z_{1}-z_{2}-z_{3}}{\alpha_{3}}\right)
\end{aligned}
$$

for every $z=\left(z_{1}, z_{2}, z_{3}\right) \in \mathbb{C}^{3}$ such that all the terms are well-defined. More specifically, the existence of the cumulant process is guaranteed for any $z=\left(z_{1}, z_{2}, z_{3}\right) \in \mathbb{R}^{3}$ satisfying:

(i) $\left|z_{1}\right|<\min \{|-\alpha-\beta|, \alpha-\beta\}$.

(ii) $z_{k} \in\left(-\alpha_{k}, \infty\right)$ for each $k \in\{2,3\}$.

(iii) $z_{1}-z_{2}-z_{3}<\alpha_{3}$.

Then, the characteristic functions of $X_{j}^{k}$ and $Y_{j}^{k}$ under $P_{T_{j}^{k}}^{\mathrm{d}}$ can be computed by using formulas 3.39 and 3.44 . The deterministic term $D_{j}^{k}$ is given by the relation 3.20 from which we get $\xi_{j}^{k}=-\ln \eta_{j}^{k}$. Finally, the ask, mid and bid model prices of the caplet are obtained by the valuation formulas (3.49), 3.45) and (3.50).

\subsubsection{Calibration Results}

The calibrated distribution parameters are presented in table 4.10 and 4.11 . We state the calibrated parameters of the volatility and liquidity function in table 4.12 and 4.13 The value of the objective function is

$$
O_{1}(\hat{\theta}, H)=6.597836 \cdot 10^{-05}
$$

\begin{tabular}{ll}
\hline Normal inverse Gaussian process & Value \\
\hline$\alpha$ & 7.426472 \\
$\beta$ & -1.773825 \\
$\delta$ & 6.334942 \\
\hline
\end{tabular}

Table 4.10.: Calibrated parameters of normal inverse Gaussian distribution.

The market (mid) prices of caps for strike rates up to three percent are satisfactorily fitted. Especially, the prices related to the six-month curve are well-adapted for these strike rates. For instance, we present the results for some maturities in figures 4.19 and 4.20. The relative errors are illustrated in figures C.1 and C.2 in the appendix. As in the 
4.3. Calibration of Caps in the Two-Price and Multiple-Curve Setting

\begin{tabular}{ll}
\hline Gamma process & Value \\
\hline$\alpha_{1}$ & 0.002901911 \\
$\beta_{1}$ & 0.004451095 \\
$\alpha_{2}$ & 0.003706087 \\
$\beta_{2}$ & 0.001636382 \\
$\alpha_{3}$ & 0.000387000 \\
$\beta_{3}$ & 0.006897160 \\
\hline
\end{tabular}

Table 4.11.: Calibrated parameters of the Gamma processes.

\begin{tabular}{ll}
\hline Volatility structure parameter & Value \\
\hline$a_{\mathrm{d}}$ & $1.000 \cdot 10^{-09}$ \\
$a_{1 \mathrm{~d}}$ & $-1.929 \cdot 10^{-05}$ \\
$a_{21}$ & $1.197 \cdot 10^{-05}$ \\
\hline
\end{tabular}

Table 4.12.: Calibrated parameters of volatility structure.

single-curve setting, we emphasise that the discrepancies in the calibrations' outcomes result from the very small values of the related prices together with the high complexity of the framework.

The calibrated parameter $\gamma$ with the corresponding value of the objective function $\mathrm{O}_{2}$ is listed in table 4.14 . The family $\left(\Psi_{\gamma}^{\mathrm{mmv}}\right)_{\gamma \geq 0}$ of MINMAXVAR distortion functions is used for the calibration.

The density functions are plotted in figures 4.21 and 4.22 . Three paths of the process $L^{1}$ are illustrated in figure 4.23 . The corresponding sample paths of the second and third component of $L$ are presented in figures 4.24 and 4.25 . We use the R-package 'GeneralizedHyperbolic' created by Scott [89]. 


\begin{tabular}{ll}
\hline Liquidity function parameter & Value \\
\hline$b^{1}$ & 0.0062280800 \\
$\sigma^{1}$ & 0.0003903129 \\
$b^{2}$ & 0.0083791380 \\
$\sigma^{2}$ & 0.0005892760 \\
\hline
\end{tabular}

Table 4.13.: Calibrated parameters of liquidity function.

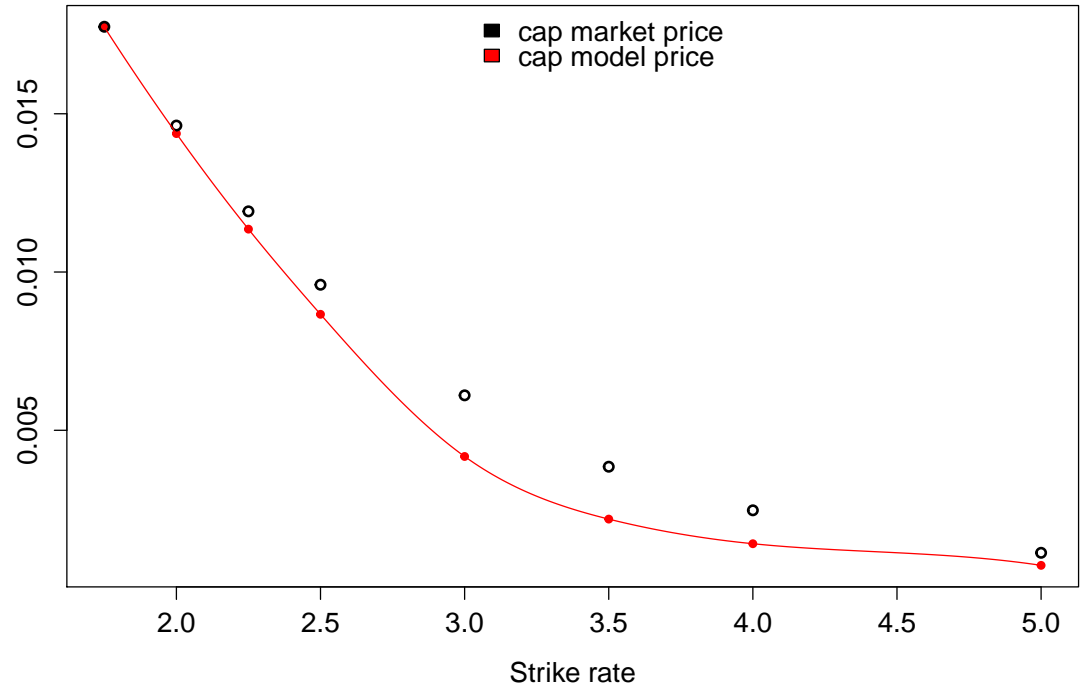

Figure 4.19.: Calibration of (mid) cap prices for maturity of 3 years.

\begin{tabular}{lll}
\hline Maturity & Parameter & Objective function \\
\hline 3.00 Year & 0.001 & $1.37 \cdot 10^{-07}$ \\
\hline
\end{tabular}

Table 4.14.: Calibrated parameter $\gamma$ for maturity $T=3$. 


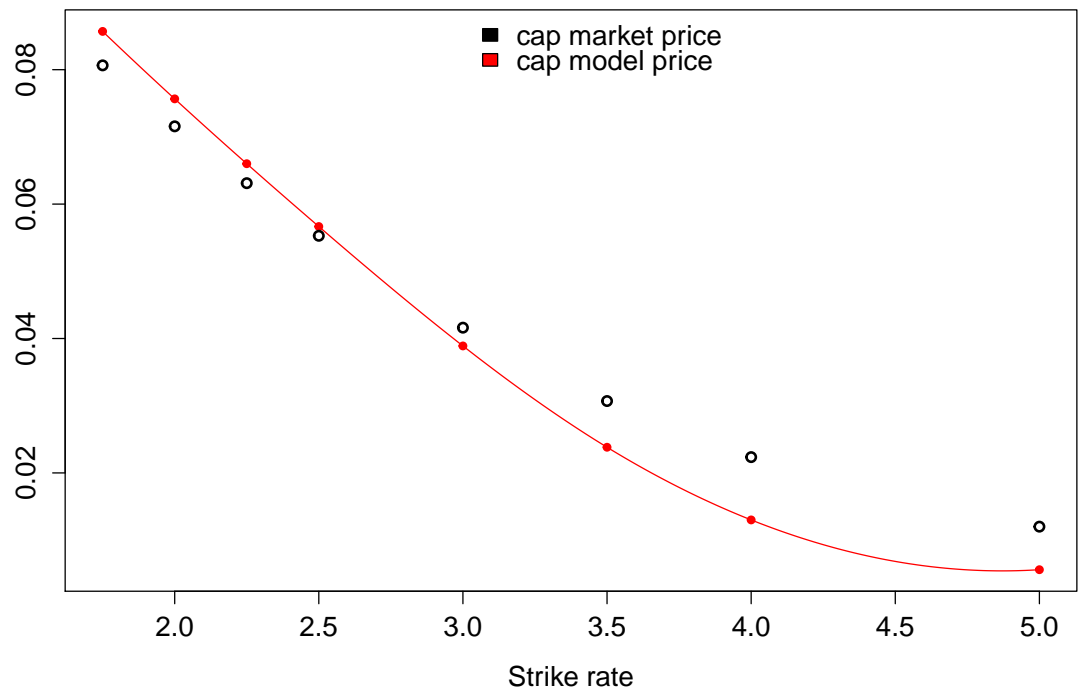

Figure 4.20.: Calibration of (mid) cap prices for maturity of 6 years.

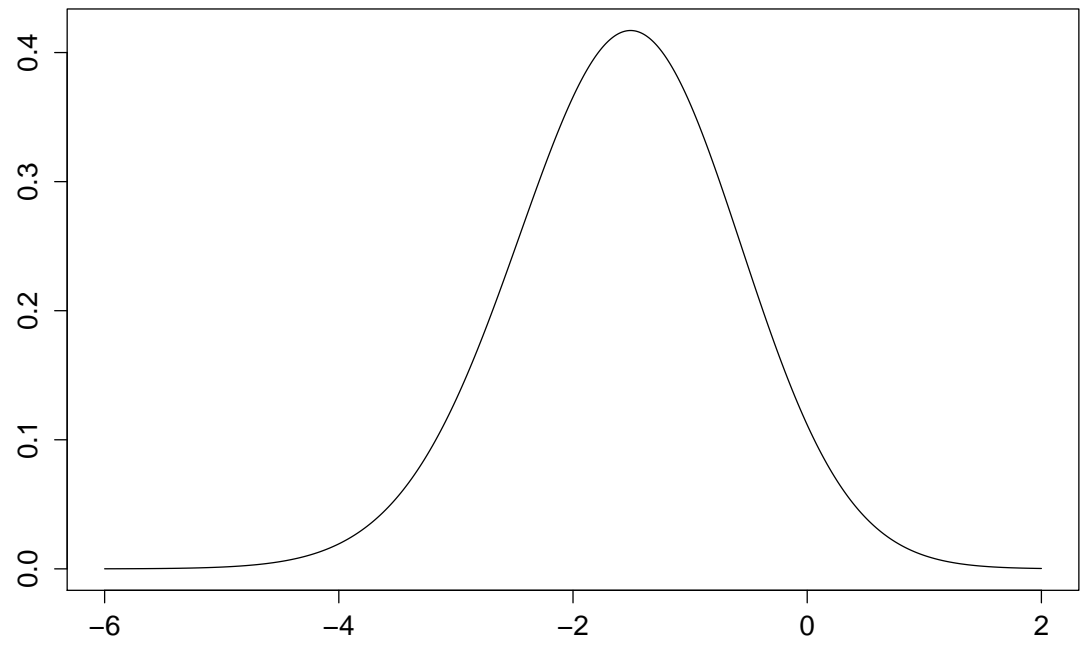

Figure 4.21.: Density function of normal inverse Gaussian distribution for the calibrated parameters. 


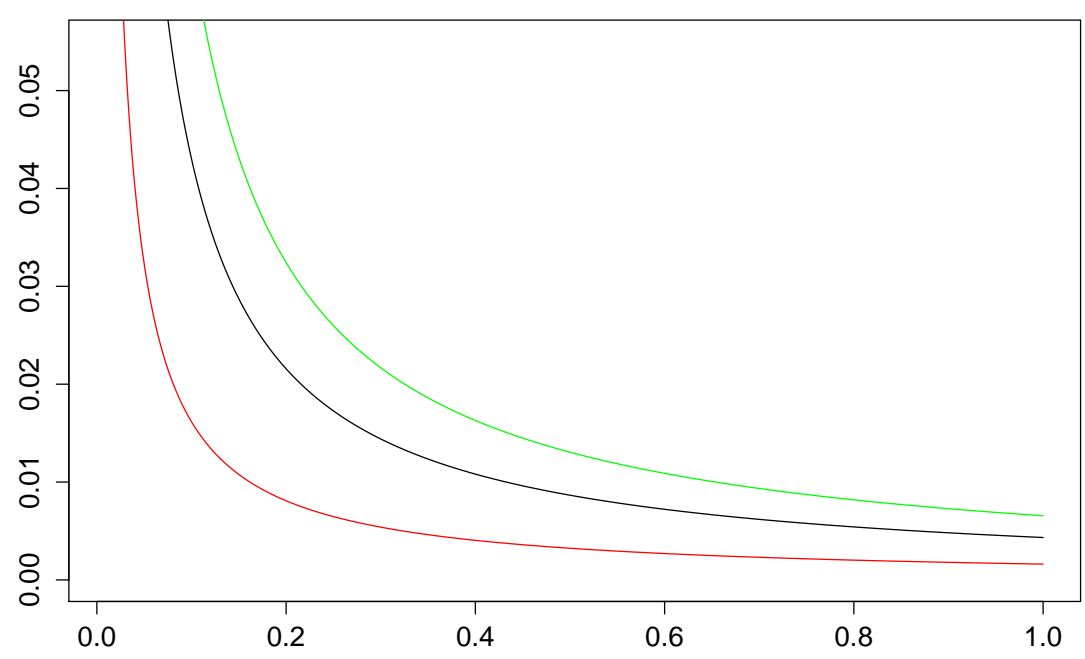

Figure 4.22.: Density function of Gamma distribution with calibrated parameters: $\alpha_{1}=0.002901911, \beta_{1}=0.004451095(\bullet) \alpha_{2}=0.003706087, \beta_{2}=$ 0.001636382 and $(\bullet) \alpha_{3}=0.000387000, \beta_{3}=0.006897160$.

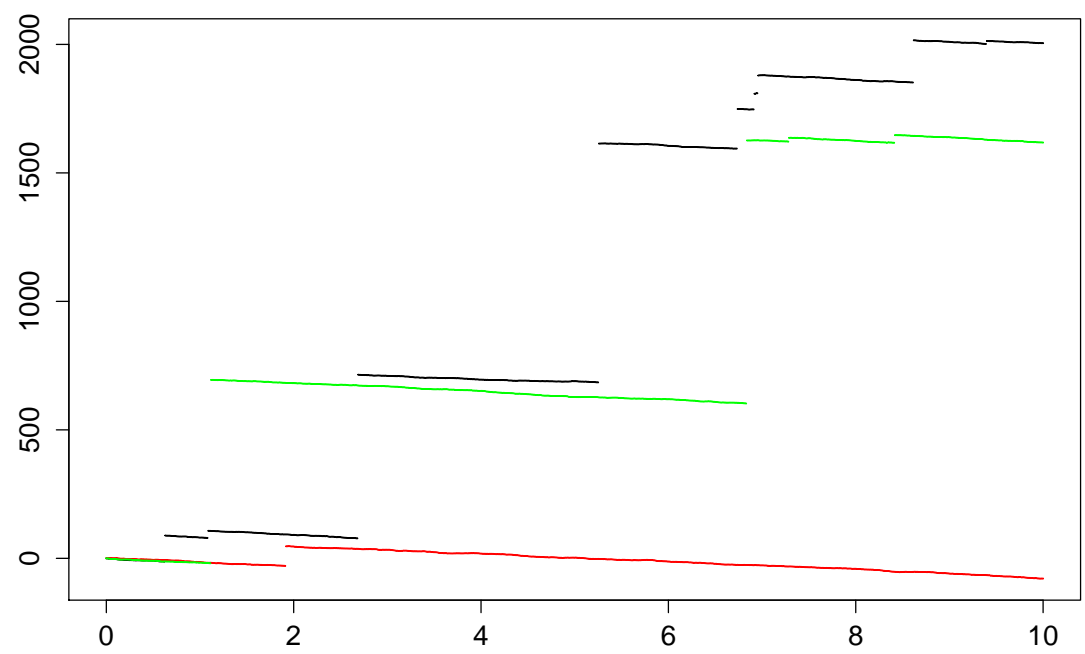

Figure 4.23.: Sample paths of $L^{1}$ with calibrated parameters. 


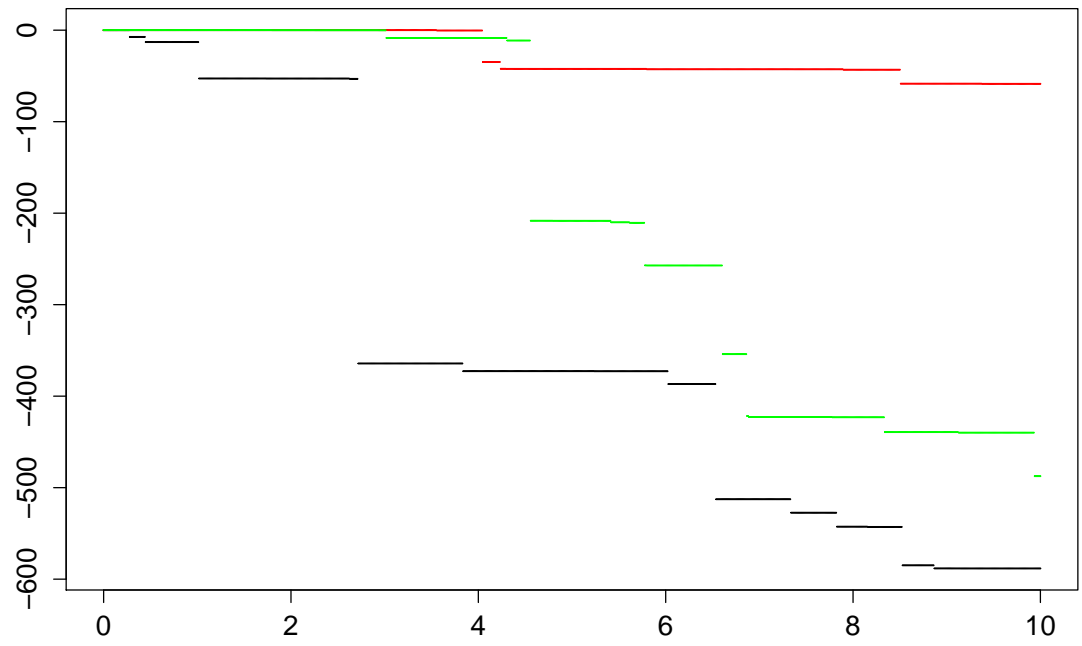

Figure 4.24.: Sample paths of $L^{2}$ with calibrated parameters.

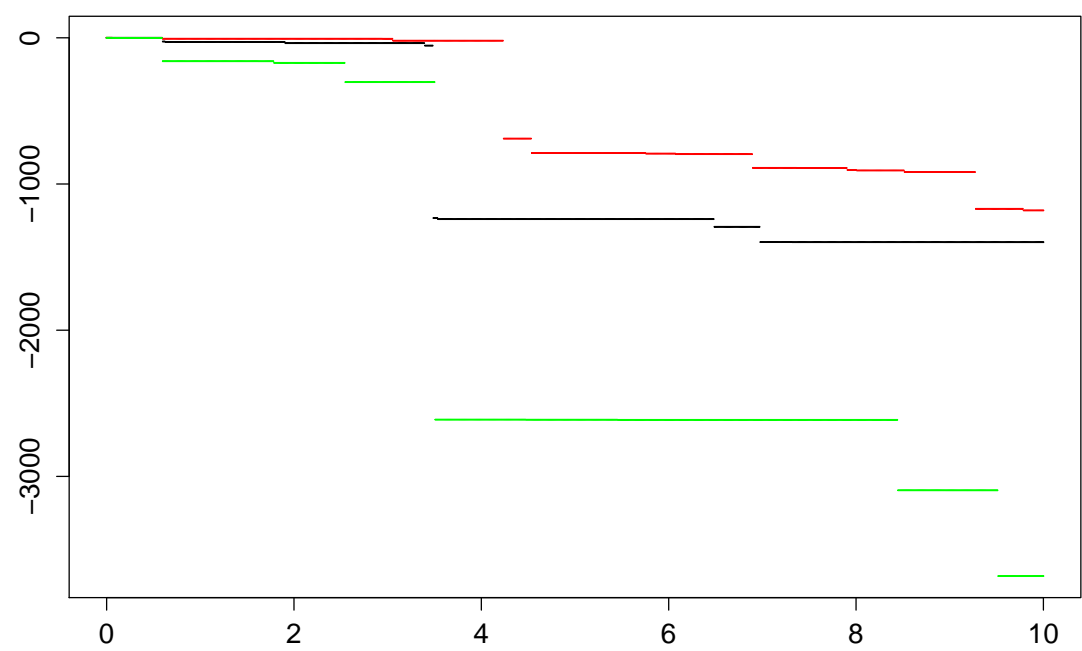

Figure 4.25.: Sample paths of $L^{3}$ with calibrated parameters. 



\section{CALIBRATION RESULTS OF MODEL FRAMEWORK (I)}

\section{Relative errors of cap (3M) market and model prices}

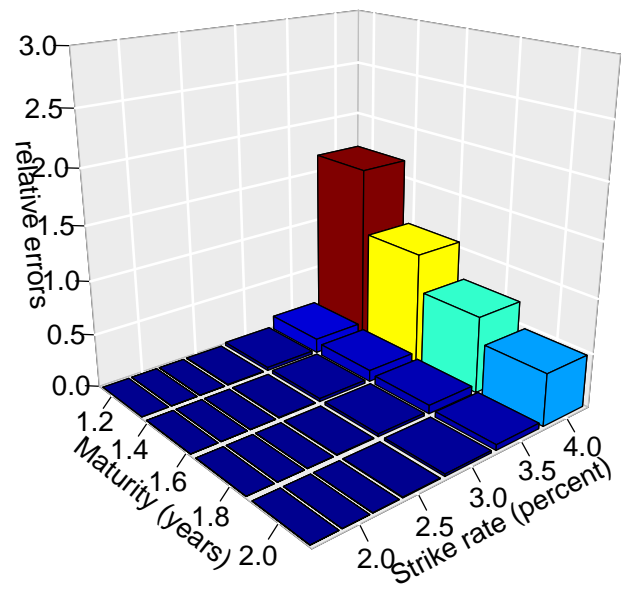

Figure A.1.: Relative errors of cap. 
A. Calibration Results of Model Framework (I)

Relative errors of cap (6M) market and model prices

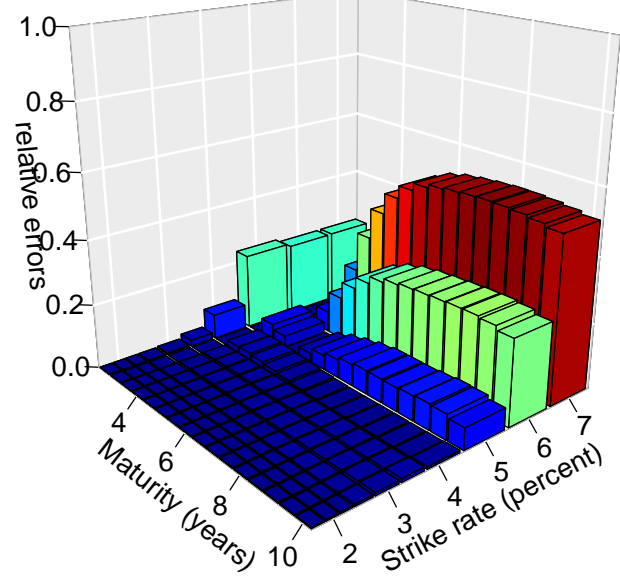

Figure A.2.: Relative errors of cap. 
CALIBRATION RESULTS OF MODEL FRAMEWORK (II)

Relative errors of cap (3M) market and model prices

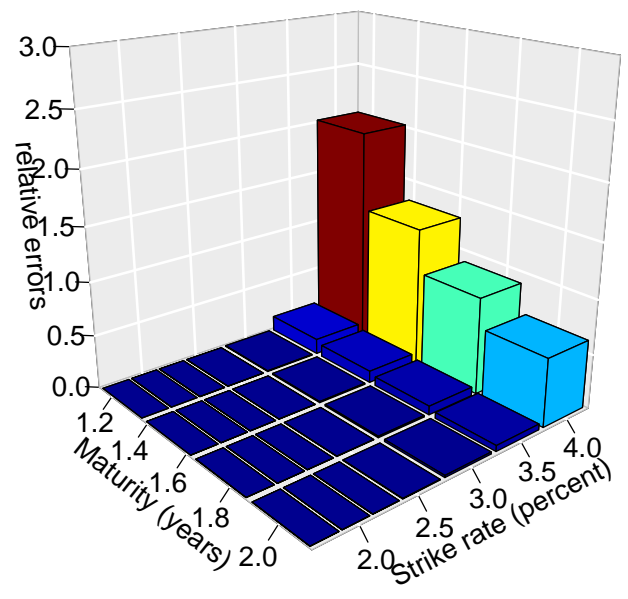

Figure B.1.: Relative errors of cap. 
B. Calibration Results of Model Framework (II)

Relative errors of cap (6M) market and model prices

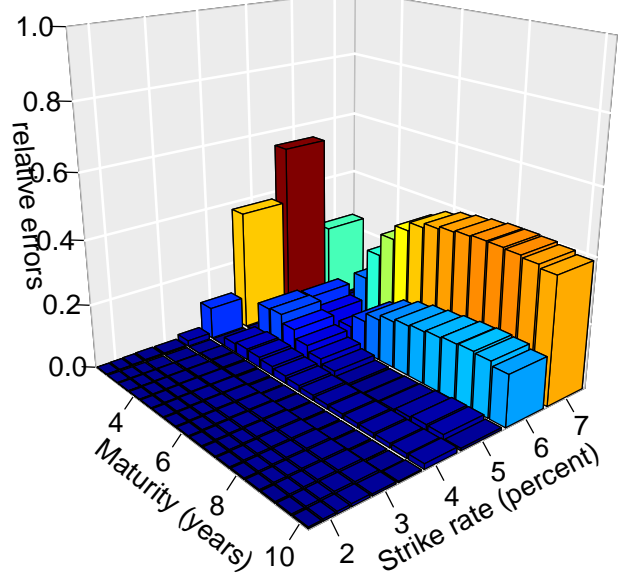

Figure B.2.: Relative errors of cap. 
Relative errors of cap (3M) market and model prices

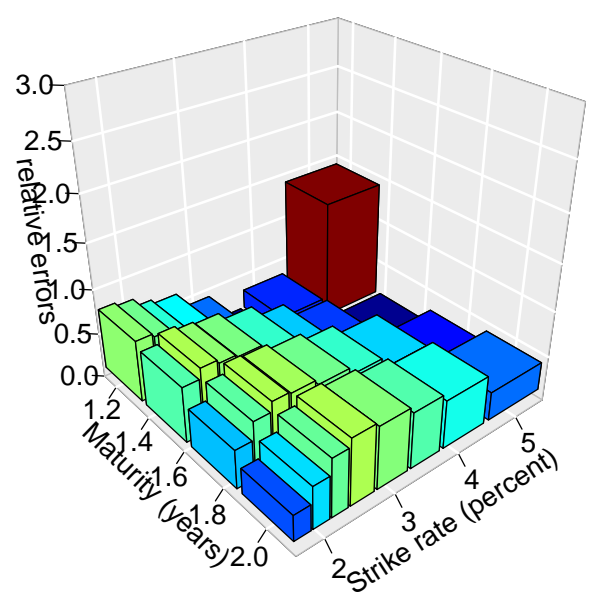

Figure C.1.: Relative errors of cap. 
C. Calibration Results in the Multiple-Curve Setting

Relative errors of cap (6M) market and model prices

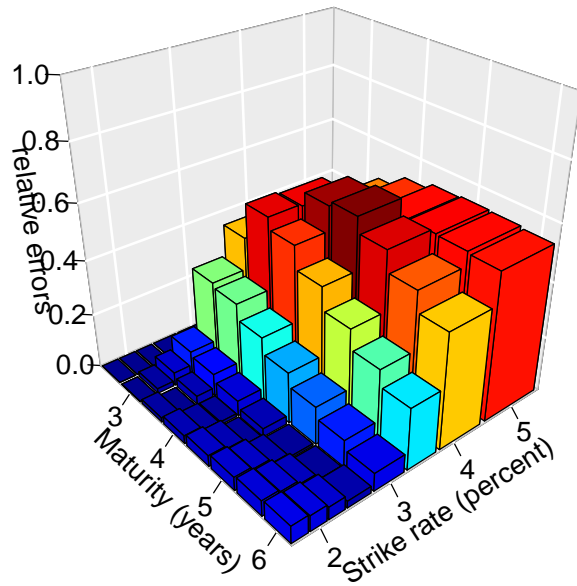

Figure C.2.: Relative errors of cap. 


\section{BIBLIOGRAPHY}

[1] Carlo Acerbi and Giacomo Scandolo. Liquidity Risk Theory and Coherent Measures of risk. Quantitative Finance, 8(7):681-692, 2008.

[2] Ferdinando M. Ametrano and Marco Bianchetti. Bootstrapping the Illiquidity: Multiple Yield Curves Construction for Market Coherent Forward Rates Estimation. Risk Books, Incisive Media, 2009.

[3] Ferdinando M. Ametrano and Marco Bianchetti. Everything You Always Wanted to Know About Multiple Interest Rate Curve Bootstrapping But Were Afraid to Ask. Available at SSRN 2219548, 2013.

[4] Philippe Artzner, Freddy Delbaen, Jean-Marc Eber, and David Heath. Coherent Measures of Risk. Risk management: value at risk and beyond, page 145, 2002.

[5] Louis Bachelier. Théorie de la Spéculation. Gauthier-Villars, 1900.

[6] Ole Barndorff-Nielsen and Christian Halgreen. Infinite Divisibility of The Hyperbolic and Generalized Inverse Gaussian Distributions. Probability Theory and Related Fields, 38(4):309-311, 1977.

[7] Ole E. Barndorff-Nielsen. Processes of Normal Inverse Gaussian Type. Finance and stochastics, 2(1):41-68, 1997.

[8] Ole E. Barndorff-Nielsen and Neil Shephard. Modelling by Lévy Processess For Financial Econometrics. Springer, 2001.

[9] Heinz Bauer. Wahrscheinlichkeitstheorie. De Gruyter Lehrbuch. Walter de Gruyter, 1991.

[10] Marco Bianchetti. Two Curves, One Price. Risk, 23(8):66, 2010.

[11] Marco Bianchetti and Mattia Carlicchi. Interest Rates After the Credit Crunch: Multiple Curve Vanilla Derivatives and SABR. Available at SSRN 1783070, 2011. 
Bibliography

[12] Tomasz Bielecki and Marek Rutkowski. Credit Risk: Modeling, Valuation and Hedging. Springer Science \& Business Media, 2002.

[13] Patrick Billingsley. Probability and Measure. John Wiley \& Sons, third edition, 1995.

[14] Fischer Black and Myron Scholes. The Pricing of Options and Corporate Liabilities. The journal of political economy, pages 637-654, 1973.

[15] Alan Brace, Dariusz Gątarek, and Marek Musiela. The Market Model of Interest Rate Dynamics. Mathematical finance, 7(2):127-155, 1997.

[16] Pierre Brémaud and Marc Yor. Changes of Filtrations and of Probability Measures. Zeitschrift für Wahrscheinlichkeitstheorie und verwandte Gebiete, 45(4):269295, 1978.

[17] Damiano Brigo and Fabio Mercurio. Interest Rate Models - Theory and Practice: With Smile, Inflation and Credit. Springer Finance. Springer, second edition, 2006.

[18] Markus K. Brunnermeier. Deciphering the Liquidity and Credit Crunch 2007-08. Technical report, National Bureau of Economic Research, 2008.

[19] Markus K. Brunnermeier and Lasse Heje Pedersen. Market Liquidity and Funding Liquidity. Review of Financial studies, 22(6):2201-2238, 2009.

[20] Alexander Cherny. Weighted V@R and its Properties. Finance and Stochastics, 10 (3):367-393, 2006.

[21] Alexander Cherny and Dilip Madan. New Measures for Performance Evaluation. Review of Financial Studies, 22(7):2571-2606, 2009.

[22] Alexander Cherny and Dilip Madan. Markets as a Counterparty: An Introduction to Conic Finance. International Journal of Theoretical and Applied Finance, 13(08): 1149-1177, 2010.

[23] Stéphane Crépey, Zorana Grbac, and Hai-Nam Nguyen. A Multiple-Curve HJM Model of Interbank Risk. Mathematics and Financial Economics, 6(3):155-190, 2012 .

[24] Freddy Delbaen. Coherent Risk Measures on General Probability Spaces. In Advances in finance and stochastics, pages 1-37. Springer, 2002.

[25] Freddy Delbaen and Walter Schachermayer. The Mathematics of Arbitrage. Springer Science \& Business Media, 2006. 
[26] Claude Dellacherie and Paul-André Meyer. Probability and Potential. Paris: Hermann, 1978.

[27] Rick Durrett. Probability: Theory and Examples. Cambridge university press, 2010.

[28] Ernst Eberlein. Application of Generalized Hyperbolic Lévy Motions to Finance. In Lévy processes, pages 319-336. Springer, 2001.

[29] Ernst Eberlein. Jump-Type Lévy Processes. In Handbook of Financial Time Series, pages 439-455. Springer, 2009.

[30] Ernst Eberlein. Valuation in Illiquid Markets. Procedia Economics and Finance, 29: 135-143, 2015.

[31] Ernst Eberlein and Ernst August v. Hammerstein. Generalized Hyperbolic and Inverse Gaussian Distributions: Limiting Cases and Approximation of Processes. In Seminar on Stochastic Analysis, Random Fields and Applications IV, pages 221-264. Springer, 2004.

[32] Ernst Eberlein and Ulrich Keller. Hyperbolic Distributions in Finance. Bernoulli, pages 281-299, 1995.

[33] Ernst Eberlein and Wolfgang Kluge. Exact Pricing Formulae for Caps and Swaptions in a Lévy Term Structure Model. Journal of Computational Finance, 9(2):99-125, 2006 .

[34] Ernst Eberlein and Wolfgang Kluge. Calibration of Lévy Term Structure Models. In Advances in mathematical finance, pages 147-172. Springer, 2007.

[35] Ernst Eberlein and Dilip Madan. Unbounded Liabilities, Capital Reserve Requirements and the Taxpayer Put Option. Quantitative Finance, 12(5):709-724, 2012.

[36] Ernst Eberlein and Fehmi Özkan. The Defaultable Lévy Term Structure: Ratings and Restructuring. Mathematical Finance, 13(2):277-300, 2003.

[37] Ernst Eberlein and Fehmi Özkan. The Lévy Libor Model. Finance and Stochastics, 9(3):327-348, 2005.

[38] Ernst Eberlein and Sebastian Raible. Term Structure Models Driven by General Lévy Processes. Mathematical Finance, 9(1):31-53, 1999.

[39] Ernst Eberlein, Jean Jacod, and Sebastian Raible. Lévy Term Structure Models: No-Arbitrage and Completeness. Finance and Stochastics, 9(1):67-88, 2005. 
Bibliography

[40] Ernst Eberlein, Kathrin Glau, and Antonis Papapantoleon. Analysis of Fourier Transform Valuation Formulas and Applications. Applied Mathematical Finance, 17 (3):211-240, 2010.

[41] Ernst Eberlein, Zorana Grbac, and Thorsten Schmidt. Market Models for CDOs Driven by Time-Inhomogeneous Lévy Processes. arXiv preprint, 2010.

[42] Ernst Eberlein, Zorana Grbac, and Thorsten Schmidt. Market Models for Credit Risky Portfolios Driven by Time-Inhomogeneous Lévy Processes. Available at SSRN $2137607,2012$.

[43] Jürgen Elstrodt. Maß-und Integrationstheorie. Springer-Verlag, sixth edition, 2008.

[44] Damir Filipovic. Term-Structure Models: A Graduate Course. Springer Finance. Springer, 2009.

[45] Damir Filipović and Anders B. Trolle. The Term Structure of Interbank Risk. Journal of Financial Economics, 109(3):707-733, 2013.

[46] Hans Föllmer and Alexander Schied. Stochastic Finance: An Introduction in Discrete Time. Walter de Gruyter, 2011.

[47] George Forsythe, Cleve Moler, and Michael Malcolm. Computer Methods for Mathematical Computations. Prentice-Hall, 1977.

[48] Sergey Foss, Dmitry Korshunov, and Stan Zachary. An Introduction to Heavy-Tailed and Subexponential Distributions. Springer, 2011.

[49] Helyette Geman, Nicole El Karoui, and Jean-Charles Rochet. Changes of Numeraire, Changes of Probability Measure and Option Pricing. Journal of Applied probability, pages 443-458, 1995.

[50] Zorana Grbac. Credit Risk in Lévy LIBOR Modeling: Rating Based Approach. PhD thesis, Freiburg (Breisgau), Univ., Diss., 2010, 2009.

[51] Patrick S. Hagan and Graeme West. Methods for Constructing a Yield Curve. Wilmott Magazine, May, pages 70-81, 2008.

[52] David Heath, Robert Jarrow, and Andrew Morton. Bond Pricing and the Term Structure of Interest Rates: A New Methodology for Contingent Claims Valuation. Econometrica: Journal of the Econometric Society, pages 77-105, 1992.

[53] Marc Henrard. The Irony in the Derivatives Discounting. Available at SSRN 970509, 2007. 
[54] Marc Henrard. The Irony in Derivatives Discounting Part II: The Crisis. Wilmott Journal, 2(6):301-316, 2010.

[55] John C. Hull. Options, Futures, and Other Derivatives. Prentice Hall, eighth edition, 2012.

[56] Jean Jacod and Albert Shiryaev. Limit Theorems for Stochastic Processes. Grundlehren der mathematischen Wissenschaften. Springer-Verlag, second edition, 2003.

[57] Farshid Jamshidian. Valuation of Credit Default Swaps and Swaptions. Finance and Stochastics, 8(3):343-371, 2004.

[58] Robert A. Jarrow and Alexandre F. Roch. Liquidity Risk and the Term Structure of Interest Rates. Mathematics and Financial Economics, 9(1):57-83, 2015.

[59] Monique Jeanblanc and Yann Le Cam. Immersion Property and Credit Risk Modelling. In Optimality and Risk-Modern Trends in Mathematical Finance, pages 99132. Springer, 2009.

[60] Olav Kallenberg. Foundations of Modern Probability. Springer Science \& Business Media, second edition, 2002.

[61] Jan Kallsen and Albert N. Shiryaev. The Cumulant Process and Esscher's Change of Measure. Finance and Stochastics, 6(4):397-428, 2002.

[62] Masaaki Kijima, Keiichi Tanaka, and Tony Wong. A Multi-Quality Model of Interest Rates. Quantitative Finance, 9(2):133-145, 2009.

[63] Achim Klenke. Wahrscheinlichkeitstheorie, volume 1. Springer, 2006.

[64] Wolfgang Kluge. Time-Inhomogeneous Lévy Processes in Interest Rate and Credit Risk Models. PhD thesis, Universitätsbibliothek Freiburg, 2005.

[65] Shigeo Kusuoka. A Remark on Default Risk Models. In Advances in mathematical economics, pages 69-82. Springer, 1999.

[66] Dilip Madan and Frank Milne. Option Pricing With V.G. Martingale Components. Mathematical finance, 1(4):39-55, 1991.

[67] Dilip Madan and Eugene Seneta. The Variance Gamma (VG) Model for Share Market Returns. Journal of business, pages 511-524, 1990.

[68] Dilip Madan, Peter Carr, and Eric Chang. The Variance Gamma Process and Option Pricing. European finance review, 2(1):79-105, 1998. 
Bibliography

[69] Fabio Mercurio. Interest Rates and the Credit Crunch: New Formulas and Market Models. Bloomberg portfolio research paper, 2009.

[70] Fabio Mercurio. LIBOR Market Models with Stochastic Basis. Risk Magazine, 23 (12):84-89, 2010.

[71] Fabio Mercurio. Modern LIBOR Market Models: Using Different Curves for Projecting Rates and for Discounting. International Journal of Theoretical and Applied Finance, 13(01):113-137, 2010.

[72] Robert C. Merton. Theory of Rational Option Pricing. The Bell Journal of economics and management science, pages 141-183, 1973.

[73] Nicola Moreni and Andrea Pallavicini. Parsimonious HJM Modelling for Multiple Yield Curve Dynamics. Quantitative Finance, 14(2):199-210, 2014.

[74] Massimo Morini. Solving the Puzzle in the Interest Rate Market (Part 1 and Part 2), 2009.

[75] Marek Musiela and Marek Rutkowski. Martingale Methods in Financial Modelling. Applications of mathematics. Springer, second edition, 2006.

[76] Maury Osborne. Brownian Motion in the Stock Market. Operations research, 7(2): $145-173,1959$.

[77] Andrea Pallavicini and Marco Tarenghi. Interest-Rate Modeling with Multiple Yield Curves. Available at SSRN 1629688, 2010.

[78] Michael J.D. Powell. A Fast Algorithm for Nonlinearly Constrained Optimization Calculations. In Numerical analysis, pages 144-157. Springer, 1978.

[79] Philip E. Protter. Stochastic Integration and Differential Equations, volume 21. Springer, version 2.1, second edition, 2004.

[80] Paul A. Samuelson. Rational Theory of Warrant Pricing. IMR; Industrial Management Review (pre-1986), 6(2):13, 1965.

[81] Ken-iti Sato. Lévy Processes and Infinitely Divisible Distributions. Cambridge university press, 1999 .

[82] Philipp Schönbucher. Credit Derivatives Pricing Models: Models, Pricing and Implementation. John Wiley \& Sons, 2003.

[83] Eugene Seneta. Fitting the Variance-Gamma Model to Financial Data. Journal of Applied Probability, 41:177-187, 2004. 
Bibliography

[84] Hans-Werner Sinn. Casino Capitalism: How the Financial Crisis Came About and What Needs to Be Done Now. OUP Catalogue, 2012.

[85] Karline Soetaert and Peter Herman. A Practical Guide to Ecological Modelling. Using $R$ as a Simulation Platform. Springer, 2009.

[86] Shiqi Song. Local Solution Method for the Problem of Enlargement of Filtration. arXiv preprint arXiv:1302.2862, 2013. 



\section{R-PACKAGES}

[87] Hans Werner Borchers. Package 'Pracma'. Available online, 2015.

[88] Frank E. Harrell Jr. Package 'Hmisc'. Available in cran.r-project, 2015.

[89] David Scott. Package 'GeneralizedHyperbolic'. Available online, 2015.

[90] David Scott. Package 'VarianceGamma'. Available online, 2015.

[91] Karline Soetaert. RootSolve: Nonlinear Root Finding, Equilibrium and Steady-State Analysis of Ordinary Differential Equations, 2009. R package 1.6.

[92] R Core Team. Package 'Stats'. Available in cran.r-project, 2016. 